\title{
In Vitro Studies of Protein Interactions on Substrate Supported Artificial Membranes
}

\author{
Dissertation \\ zur Erlangung des \\ mathematisch-naturwissenschaftlichen Doktorgrades \\ „Doctor rerum naturalium“ \\ der Georg-August-Universität Göttingen \\ im Promotionsprogramm: Physics of Biological and Complex Systems \\ der Georg-August University School of Science (GAUSS) \\ vorgelegt von \\ Daniela Morick \\ aus Haldensleben
}

Göttingen, 2012 


\section{Betreuungsausschuss}

Prof. Dr. Claudia Steinem, Institut für Organische und Biomolekulare Chemie Prof. Dr. Kai Tittmann, Institut für Bioanalytik

Prof. Dr. Jörg Schroeder, Institut für PhysikalischeChemie

\section{Mitglieder der Prüfungskommission}

Referentin: $\quad$ Prof. Dr. Claudia Steinem

Korreferenten: Prof. Dr. Kai Tittmann

Prof. Dr. Jörg Schroeder

Weitere Mitglieder der Prüfungskommission:

Prof. Dr. Ulf Diederichsen

Prof. Dr. Sarah Köster

Priv.-Doz. Dr. Daniel Werz

Tag der mündlichen Prüfung: 23.01.2013 
Meiner Familie

"Alles, was an Großem in der Welt geschah, vollzog sich zuerst in der Phantasie des Menschen."

- Astrid Lindgren 



\section{Abstract}

Protein interactions that take place at the interface of a membrane are abundant within cellular organisms. Hence, surface coupled protein interactions are a matter of ongoing scientific investigation. In this work, elected protein interactions were studied at the interface of artificial membranes, attempting to create model systems that would best mimic the natural protein environment.

In the first part of this work (chapter 4-6), a quartz crystal microbalance (QCM) biosensor assay based on solid supported membranes (SSMs) was established to quantify the inter-action of C-terminal polycystin-2 (cPC2) with its putative interaction partners $C$ terminal polycystin-1 (cPC1) and PIGEA14. It was found that the affinity of cPC2 to cPC1 was three times higher in the presence of $\mathrm{Ca}^{2+}$, leading to the assumption that cPC2 forms trimers in the absence of $\mathrm{Ca}^{2+}$. Based on the observation that the kinetic rate constants are $\mathrm{Ca}^{2+}$ dependent, a binding model was developed that describes a three step cPC2 binding in the absence and a one step binding in the presence of $\mathrm{Ca}^{2+}$. Following a hypothesis that had been suggested earlier, the interaction of cPC2 with PIGEA14 was investigated as a function of cPC2 pseudophosphorylation at Ser ${ }^{812}$. It was found that the affinity of the pseudophosphorylated mutant cPC2S812D to PIGEA14 was indeed reduced two fold compared to cPC2wt.

The second part of the thesis (chapter 7 and 8) dealt with investigating the interaction of filamentous actin (F-actin) with SSMs and pore spanning membranes (PSMs) on porous aluminum or silicon nitride based surfaces by means of optical waveguide spectroscopy (OWS) and confocal laser scanning microscopy (CLSM). Mimicking the structure of cellular microvilli, specific F-actin adsorption within and atop porous anodic aluminum oxide (AAO) films could be controlled using different functionalization strategies. The impact of a membrane bound F-actin network on the tension and viscoelastic properties of PSMs was investigated by means of atomic force microscopy (AFM). While the membrane tension remained almost unaltered, the origination of a viscoelastic membrane properties was caused by the attached F-actin network. 



\section{Zusammenfassung}

Da eine Vielzahl von Proteininteraktionen innerhalb zellulärer Organismen an der Grenzfläche zu Membranen stattfindet, ist die Untersuchung dieser Prozesse von großem wissenschaftlichem Interesse. Ziel dieser Arbeit war es Modellsysteme basierend auf artifiziellen Membranen zu entwickeln, mit deren Hilfe die Untersuchung ausgewählter Proteininteraktionen ermöglicht werden konnte.

Im ersten Abschnitt dieser Arbeit (Kapitel 4-6) wurde ein Biosensorassay basierend auf festköperunterstützten Membranen entwickelt, der die Quantifizierung der Interaktion von C-Polycystin-2 (cPC2) mit seinen Interaktionspartnern C-Polycystin-1 (cPC1) und PIGEA14 mittels der Quarzmikrowaagetechnik ermöglichte. Aufgrund der Tatsache, dass die Affinität von cPC2 zu cPC1 in Anwesenheit von $\mathrm{Ca}^{2+}$ dreifach höher war, wurde eine $\mathrm{Ca}^{2+}$ abhängige Trimerisierung von cPC2 postuliert. Die Unterschiede der ermittelten kinetischen Koeffizienten führten zur Entwicklung eines Bindunsgmodells, welches die dreistufige Adsorption von cPC2 an cPC1 in Abwesenheit bzw. einstufige Adsorption in Anwesenheit von $\mathrm{Ca}^{2+}$ implizierte. Im Falle der Interaktion von cPC2 mit PIGEA14 wurde die Abhänigkeit der cPC2 Bindung von der Pseudophosphorylierung des Proteins an Ser ${ }^{812}$ untersucht. Es wurde festgestellt, dass die Affinität der pseudophosphorylierten Mutante cPC2S812D zu PIGEA14 zweifach niedriger war, als die von cPC2wt.

Im zweiten Abschnitt der Arbeit (Kapitel 7 und 8) wurde die spezifische Wechselwirkung von filamentösem Aktin (F-Aktin) mit festkörperunterstützten und porenüberspannenden Membranen untersucht. Die kontrollierte Anbindung von F-Aktin in und auf porösen Aluminiumoxidfilmen konnte mit Hilfe verschiedener Funktionalisierungsstrategien erzielt werden. Der Einfluss eines F-Aktin Netzwerks auf die Spannung und viskoelastischen Eigenschaften porenüberspannender Membranen wurde mittels kraftmikroskopischer Studien untersucht. Es wurde nachgewiesen, dass der Einfluss von gebundenem F-Aktin auf die Membranspannung gering war, aber erst durch die F-Aktin Adhäsion viskoelastische Membraneigenschaften induziert wurden. 



\section{Table of Contents}

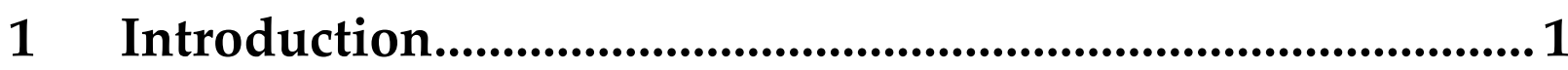

1.1 Protein Interactions in Eukaryotic Cells ...........................................................

1.2 Studying Protein-Protein Interactions.....................................................................

1.2.1 Quantifying Protein-Protein Interactions ..................................................................

1.3 Model Membranes to Characterize Proteins and Protein Interactions .............6

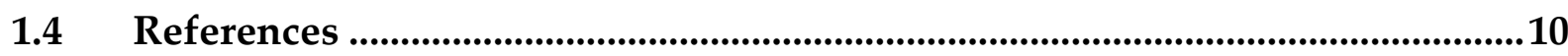

2 Scope of the Thesis ............................................................ 15

$3 \quad$ Materials and Methods .......................................................17

3.1 Experimental Methods........................................................................................17

3.1.1 Preparation of Nanoporous Anodic Aluminum Oxide Substrates .....................17

3.1.2 Functionalization Strategies …………………………………………….....2

3.2 Lipidchemical Methods .............................................................................................

3.2.1 Preparation of Lipid Films...............................................................................

3.2.2 Preparation of Unilamellar Vesicles....................................................................34

3.2.3 Solid Supported Monolayers on Functionalized Surfaces .....................................35

3.2.4 Solid Supported Bilayers on Silicon Substrates ....................................................38

3.2.5 Pore Spanning Membranes on Silicon Nitride Substrates .....................................39

3.3 Proteinbiochemical Methods......................................................................40

3.3.1 Heterologous Protein Expression in Escherichia coli ...............................................43 
3.3.2 Protein Purification and TEV Protease Cleavage ........................................... 44

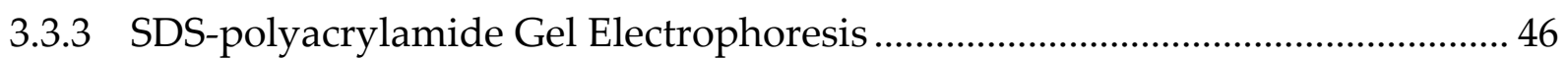

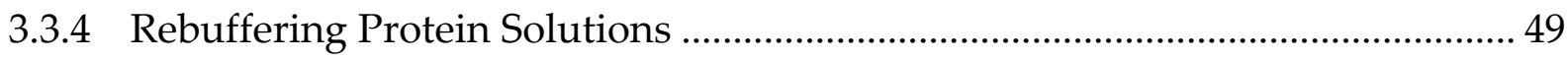

3.3.5 Quantification of Protein Concentration........................................................... 50

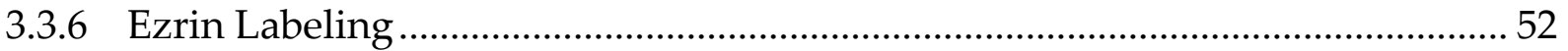

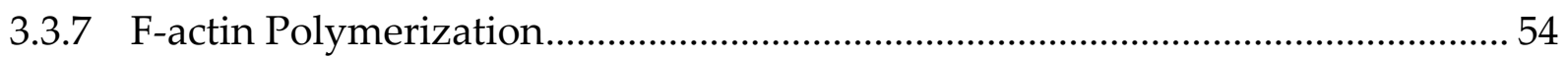

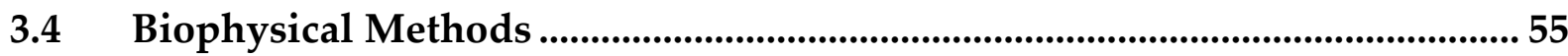

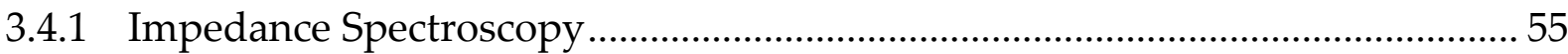

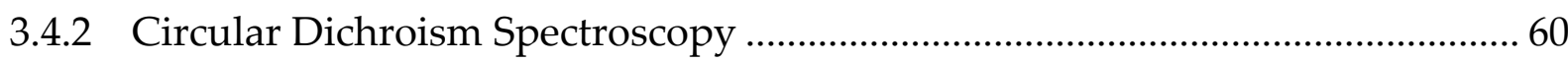

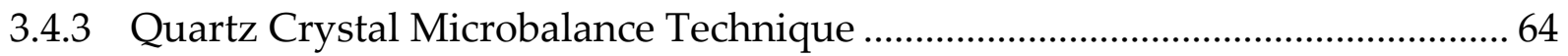

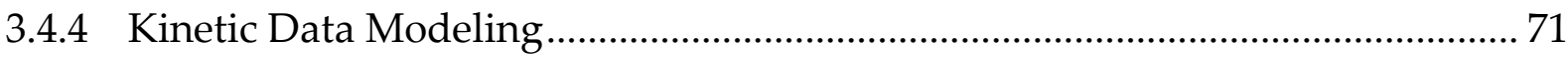

3.4.5 Surface Plasmon Resonance Spectroscopy …................................................. 73

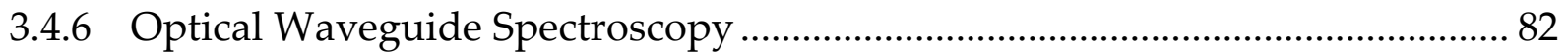

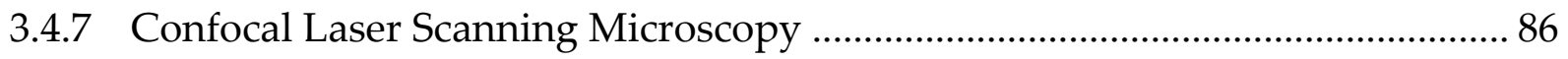

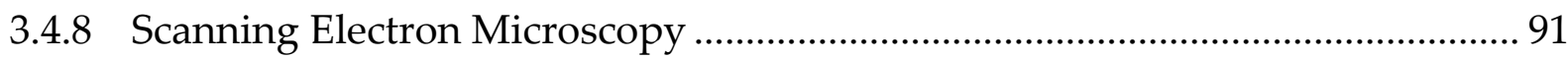

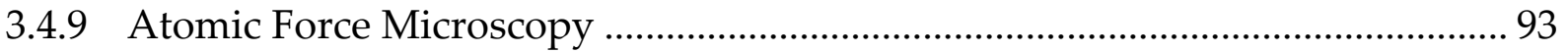

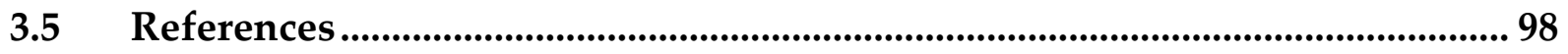

4 Biosensor Assay Development Based on Solid Supported Membranes: Studying Protein-Protein Interactions ........107

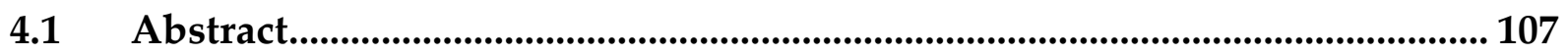

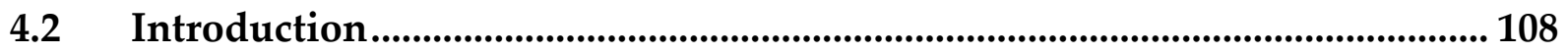

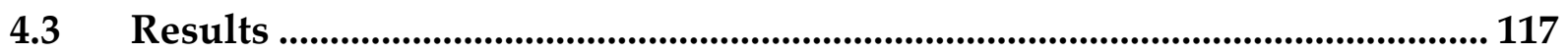


4.3.1 Protein Expression and Purification

4.3.2 Impedance and SPR Analysis of Hybrid Solid Supported Membranes on Gold

4.3.3 Fluorescence Microscopy and FRAP Analysis of Hybrid Solid Supported Membranes on Silicon 123

4.3.4 His6-tag Protein Immobilization on DOGS-NTA-Ni Containing Membranes124

4.3.5 Interaction of $\mathrm{cPC} 1$ and $\mathrm{cPC} 2$ on Solid Supported Membranes Investigated by Means of QCM and SPR 128

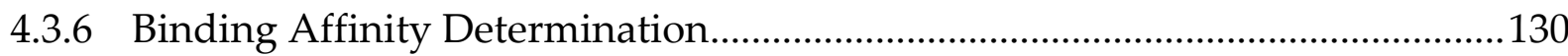

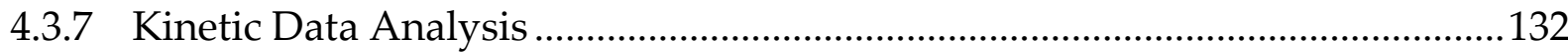

4.3.8 Influence of Mass Transport on the Kinetics of the cPC2/cPC1 Complex Formation 137

4.4 Discussion .140

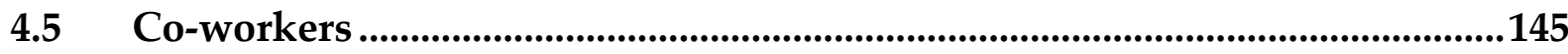

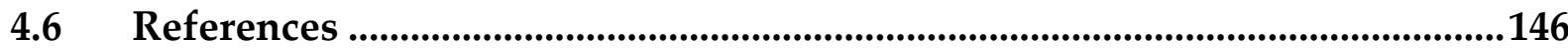

5 Quantifying the Interaction of the $C$-terminal Regions of Polycystin-2 and Polycystin-1 Attached to a Lipid Bilayer by Means of QCM......................................................................... 151

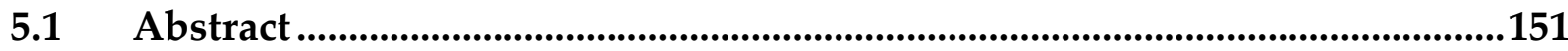

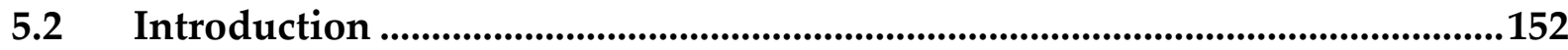

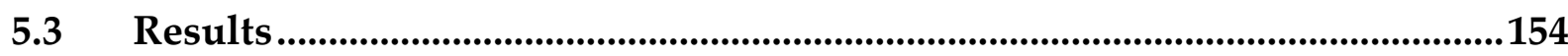

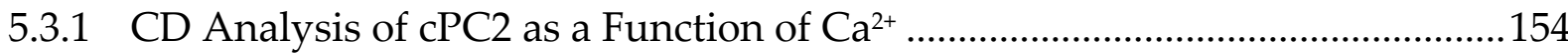

5.3.2 Immobilization of cPC1 on a Solid Supported Membrane .............................156 
5.3.3 Interaction of cPC2 with cPC1 Immobilized on a Solid Supported Membrane 158

5.3.4 Binding Affinity of the $\mathrm{cPC} 1 / \mathrm{cPC} 2$ Interaction in the Absence and Presence of $\mathrm{Ca}^{2+}$ 159

5.3.5 Kinetics of the $\mathrm{cPC} 1 / \mathrm{cPC} 2$ Interaction in the Absence and Presence of $\mathrm{Ca}^{2+} . .161$

5.4 Discussion 163

5.5 Co-workers 167

5.6 References 168

6 Phosphorylation of $C$-terminal Polycystin-2 Influences the Interaction with PIGEA14: A QCM Study Based on Solid Supported Membranes .173

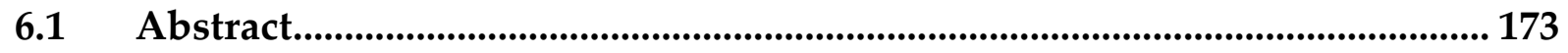

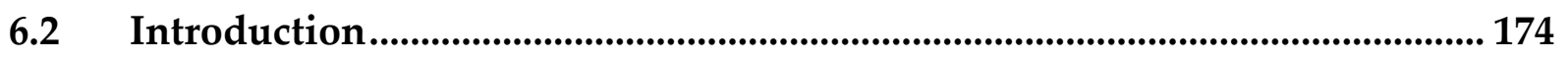

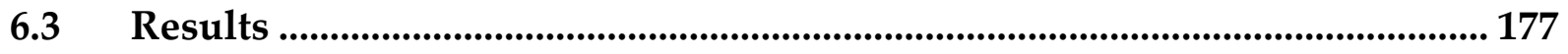

6.3.1 Expression, Purification and Characterization of PIGEA14 ........................... 177

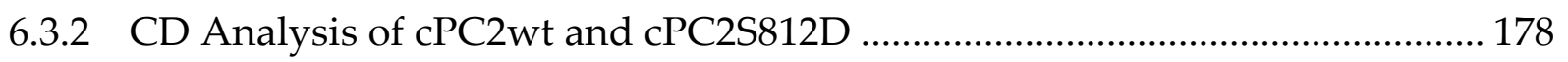

6.3.3 Immobilization of PIGEA14 on Solid Supported Membranes ........................ 179

6.3.4 Interaction of cPC2wt and cPC2S812D with PIGEA14 ................................. 181

6.3.5 Binding Affinity and Kinetics of cPC2wt and cPC2S812D ............................. 182

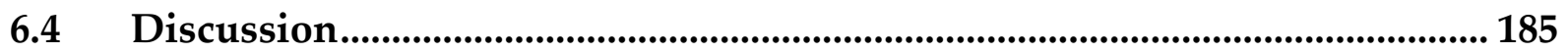

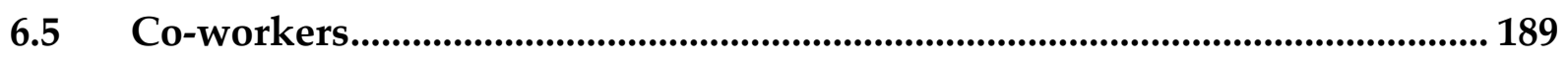

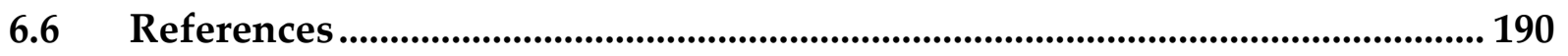


7 Directing the Adsorption of Filamentous Actin Within Surface-modified, Cylindrical Nanopores

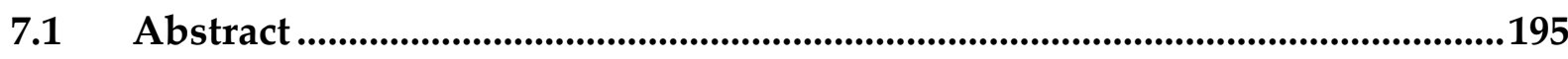

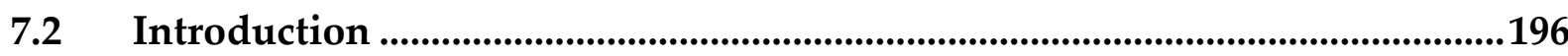

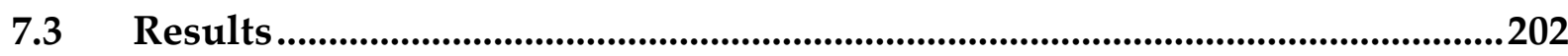

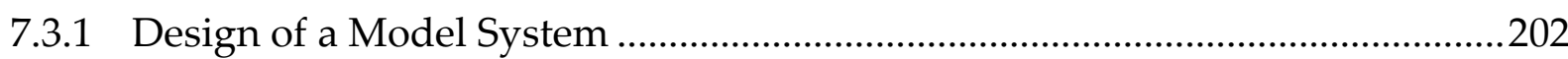

7.3.2 F-actin Adsorption Within Porous AAO Functionalized with APTES.............204

7.3.3 F-actin Adsorption Within Porous AAO with a DOEPC Containing Membrane .208

7.3.4 F-actin Adsorption Within Porous AAO with an erzin/PIP 2 Containing

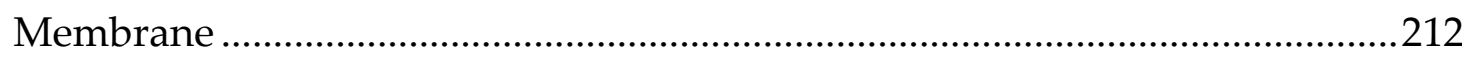

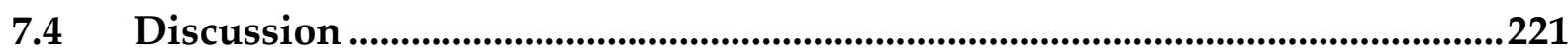

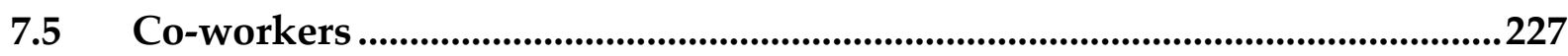

$7.6 \quad$ References ……….................................................................................................228

8 Mechanical Characterization of Filamentous Actin on Pore Spanning Membranes by Means of Force Spectroscopy 233

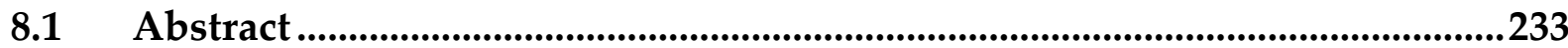

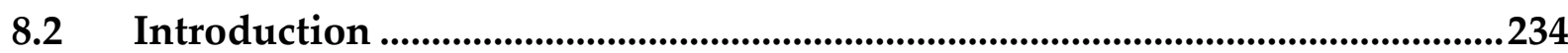

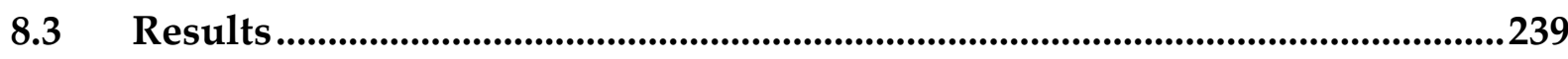

8.3.1 F-actin Binding Assay on Solid Supported Membranes ....................................239

8.3.2 F-actin Binding on Pore Suspending Membranes................................................243 
8.3.3 Force Spectroscopic Characterization of F-actin Decorated Pore Spanning

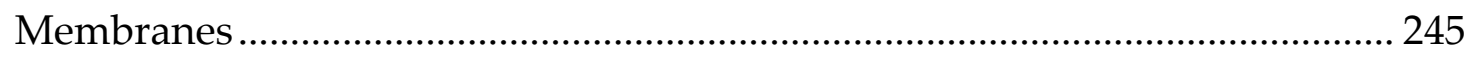

$8.4 \quad$ Discussion ..................................................................................................... 249

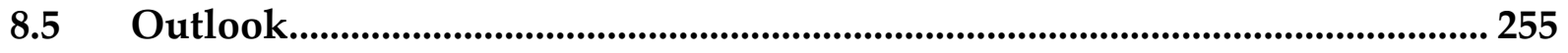

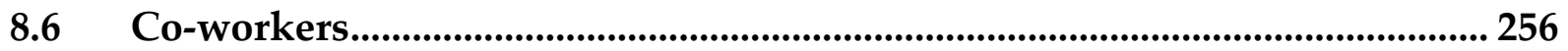

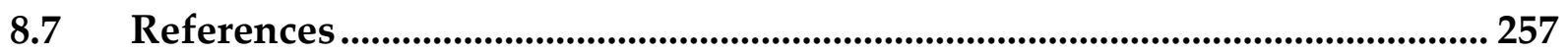

9 Conclusion and Outlook ..............................................263

$9.1 \quad$ References ............................................................................................................... 267

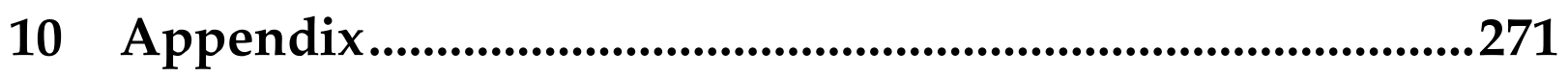

10.1 Symbols .............................................................................................................. 271

10.2 Abbreviations........................................................................................................ 275

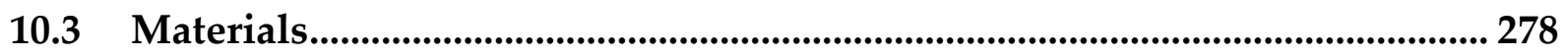

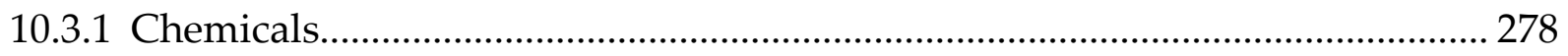

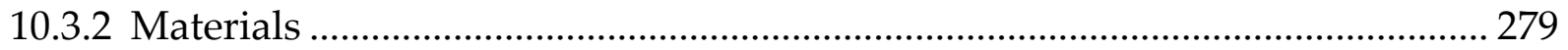

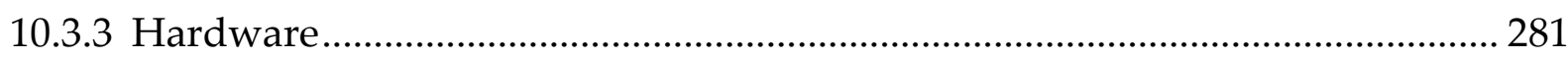






\subsection{Protein Interactions in Eukaryotic Cells}

Eukaryotic cells are composed of membrane enclosed, highly specialized organelles such as the endoplasmatic reticulum, the Golgi apparatus, mitochondria and the nucleus. This cellular compartmentalization guarantees realizing distinct cellular processes i.e. metabolism, synthesis of biomolecules and transport in a confined environment. Protein-protein interactions play fundamental roles within these cellular processes. They are involved in signal transduction, cell cycle control, DNA processing and enzyme regulation. ${ }^{1,2}$ It is of high scientific interest to understand the role of cellular protein-protein interactions in order to elucidate cellular processes, networks and the origin of diseases at the protein level. ${ }^{3}$ This knowledge would allow to specifically target the particular proteins of interest with selective drugs.

Protein interactions can be either permanent or transient. Hemoglobin, for instance can be purified as a stable assembly of four protein subunits. Transient interactions, on the contrary require certain regulatory factors. ${ }^{4}$ In general, control of the protein's oligorimeric state can be achieved by directing the encounter of interacting surfaces, by varying local protein concentrations or adapting the physicochemical environment. In the latter case, phosphorylation, changes in $\mathrm{pH}$ and temperature or the presence of certain effector molecules (i.e. $\mathrm{Ca}^{2+}$, ATP) lead to conformational changes that alter the protein's binding affinity to its interaction partner. ${ }^{4}$

Several databases concentrate on analyzing the interactome of protein-protein interactions. ${ }^{5}$ The database of interacting proteins (DIP) for instance reported that over 25,000 
proteins participate in $\sim 75,000$ interactions. ${ }^{6}$ General exemples of those interactions are antigen-antibody, hormone-receptor and enzyme-inhibitor interactions. ${ }^{7}$

Interactions between proteins are mainly driven by van der Waals forces, electrostatic forces or hydrogen bonding; the contact area of the protein-protein complex is confined to special regions on the protein surface. ${ }^{8}$ These hot spots can be identified and characterized in terms of their amino acid composition using alanine mutagenesis screening assays. It has been found that some amino acids like Trp, Tyr and Arg are highly enriched within the hot spots and often surrounded by hydrophobic areas, possibly to exclude bulk solvent. ${ }^{9}$ The size of these interfaces range between $10-40 \mathrm{~nm}^{2} .{ }^{10}$ Interestingly, the free binding energy of the protomers seems not to be correlated with the size of the complex interface. ${ }^{4}$ The identification, characterization and quantification of protein-protein interactions have been key topics of previous and current scientific studies. ${ }^{11-13}$

\subsection{Studying Protein-Protein Interactions}

There are several methods known to analyze and characterize protein interaction networks (Fig. 1.1). ${ }^{14,2}$ In order to identify putative protein interaction partners in vivo the yeast two hybrid ( $\mathrm{Y} 2 \mathrm{H})$ screening has been established as a suitable technique. The method bases on the activation of a reporter gene after interaction of two proteins that are each coupled to the binding domain (bait protein) of an upstream activation sequence and the corresponding activation domain (prey protein), respectively. Although the $\mathrm{Y} 2 \mathrm{H}$ system is widely applied, it is limited to soluble proteins. The interaction of membrane coupled proteins can be studied by means of protein fragment complementation. A protein i.e. ubiquitin is split into two inactive fragments, which are fused to the bait and the prey. Only upon interaction, the function of ubiquitin is regained, leading to the cleavage of a reporter protein. As a main disadvantage, the high number of 
false positive interactions has to be mentioned, which makes further investigations of the proposed interaction indispensable.

in vivo

in vitro

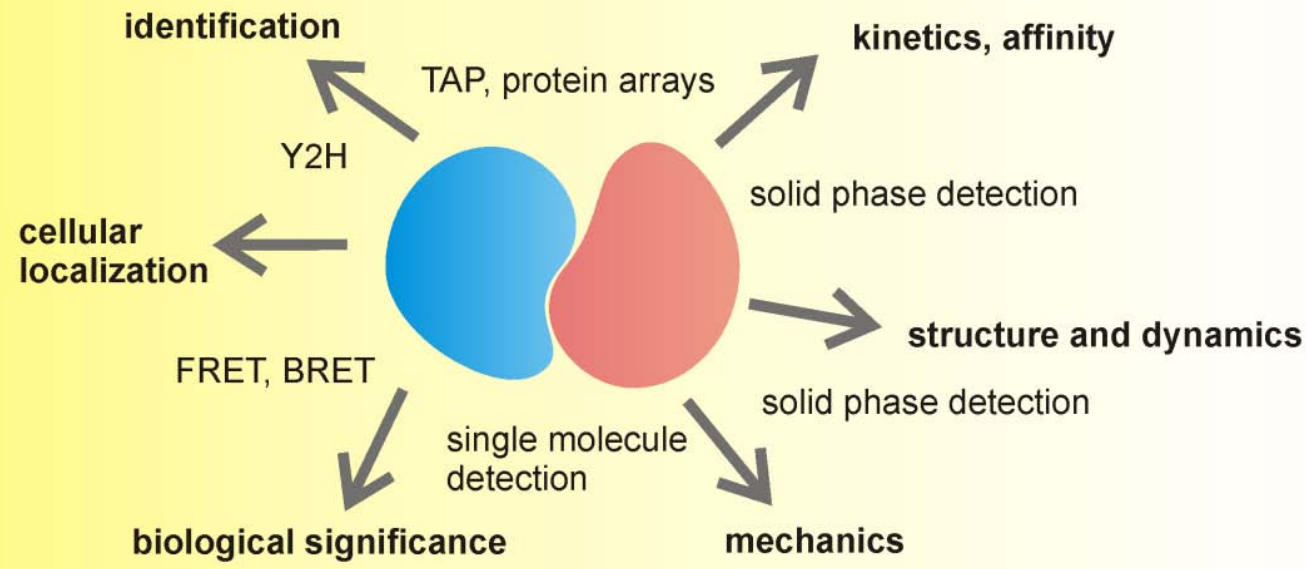

Fig. 1.1 Different levels of protein-protein interaction characterization in vivo and in vitro. ${ }^{14}$

Identification of protein interactions in vitro can be performed by means of coimmunoprecipitation (Co-IP) or tandem affinity purification (TAP). Both methods are pull-down assays that rely on affinity purification of the bait protein in complex with its interaction partners. Besides this, cross linking agents are often employed to probe protein-protein interactions or even study their subunit structure. Upon interaction, proteins are in close proximity and can thus be easily covalently linked to each other. $N$-Hydroxysuccinimide (NHS) esters are commonly used to link primary amine groups of lysine residues. Further analysis of the proteins in complex is performed by means of mass spectrometry or two dimensional gels. ${ }^{15,2}$

Besides the identification of biological relevant protein-protein interactions, the characterization of the respective interaction in terms of its thermodynamic and kinetic binding behavior is mandatory to bring further insight into the mechanism of the protein complex formation. ${ }^{13}$ 


\subsubsection{Quantifying Protein-Protein Interactions}

Methods to monitor and quantify protein-protein interactions in cellular systems are rare and mostly involve fluorescence techniques such as fluorescence resonance energy transfer (FRET) or bioluminescence resonance energy transfer (BRET), where an energy transfer between a donor and an acceptor fluorophor occurs, if the fluorophores reach a certain distance $(\sim 7 \mathrm{~nm}) \cdot{ }^{14,16}$ Another fluorescence based method that is used to monitor protein interactions in vitro is the fluorescence correlation or fluorescence crosscorrelation spectroscopy (FCS, FCCS), which allows measuring diffusion rates of proteins and their complexes. ${ }^{17}$ However, fluorescence based techniques require labeling of the proteins under investigation. In living cells, the green fluorescent protein (GFP) and other variants are often employed, with the main drawback of potentially influencing natural protein folding and thus their affinity to other proteins or biomolecules. Label free screening methods of protein interactions have thus become more and more popular. The isothermal titration calorimetry (ITC) is well known to determine binding affinities and stoichiometries of protein-protein complexes. ${ }^{18,19}$ The measuring principle relies on the detection of small thermal changes upon protein-protein complex formation in solution. Nevertheless, the drawbacks of this often applied technique include that relatively large amounts of protein are needed and only thermodynamic parameters, but no kinetic rate constants can be determined.

These drawbacks were overcome by introducing surface coupled biosensors. ${ }^{19,20}$ Theses instruments do not require protein labeling or high sample concentrations. Also, as they monitor biomolecular interactions in a time resolved manner, kinetic data evaluation is possible. As the first biosensor technique, the optical method ellipsometry was established by Trurnit et al. in 1952, to study thin film adsorption by a change of light polarization after reflection. Another setup that monitors optical thickness changes by means of thin film interference is the reflectrometric interference spectroscopy (RIfS). The surface plasmon resonance (SPR) technique was first introduced in 1983 by Liedberg et al. as a highly sensitive evanescent field biosensor. ${ }^{21}$ Since in 1990 the commer- 
cial BIACOR instrument was launched, the SPR technique became the most commonly used method to study analyte-ligand interactions. ${ }^{22}$ As an acoustic biosensor, the quartz crystal microbalance (QCM) allows detecting binding events as changes in resonance frequency and resistance of the quartz crystal resonator..$^{20,23}$

On single molecule level, surface coupled protein-protein interactions are measured by means of fluorescence techniques (i.e. total internal reflection fluorescence, TIRF) or force spectroscopy. Although the atomic force microscopy (AFM) technique relies on determining the interaction forces of protein complexes (sensitivity in $\mathrm{pN}$ range) instead of interaction energies, valuable information can be gathered in terms of the proteins reaction coordinates, interfaces that are involved in the interaction, protein unfolding and binding stoichiometry. ${ }^{24,25}$ Selected methods that allow to quantify proteinprotein interactions are schematically depicted in Fig. 1.2.

solution based

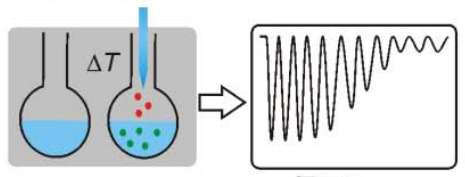

affinity interaction forces

AFM

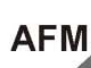

ITC

mapping, interface analysis quantification

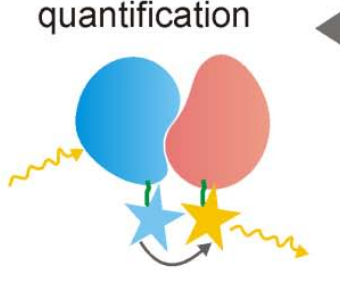

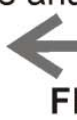

FRET,

BRET
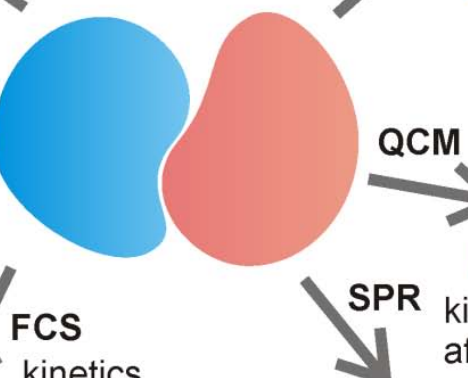
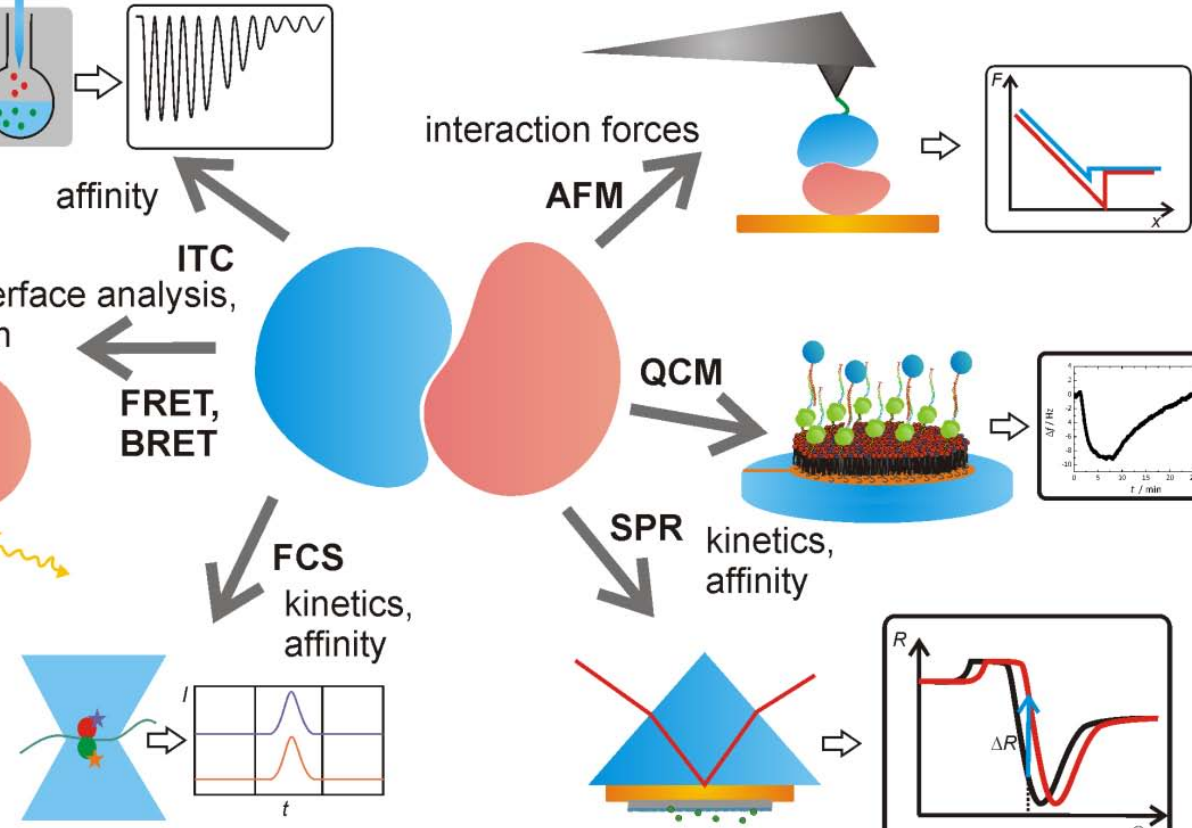
surface coupled

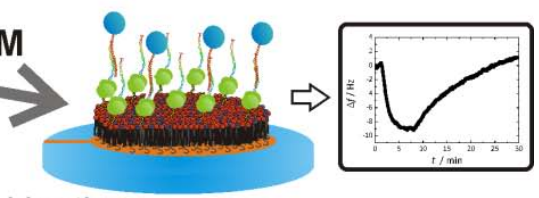

kinetics,

affinity

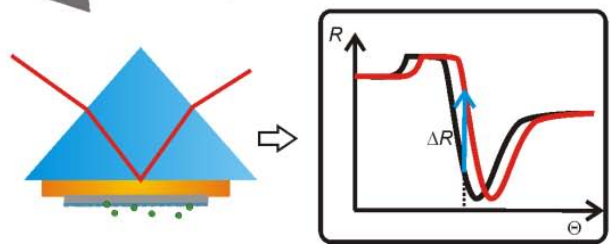

Fig. 1.2 Schematic drawing showing selected methods that allow quantifying protein-protein interaction in solution or surface coupled such as: isothermal titration calorimetry (ITC), atomic force microscopy (AFM), quartz crystal microbalance (QCM), surface plasmon resonance (SPR), fluorescence correlation spectroscopy (FCS), fluorescence or bioluminescence resonance energy transfer (FRET and BRET). 
Surface coupled biosensor techniques require modification of the transducer surface in order to specifically bind the target protein (see chapter 4.2 on page 108 for detailed description). Typical strategies to modify a metal coated transducer surface are thiols or silanes with attached head groups that are capable of exclusively binding the protein

of interest. Artificial membranes provide another possibility to couple proteins using modified phospholipid head groups with the main benefit of guaranteeing the lateral mobility of the immobilized proteins in order to obtain the best possible conditions to mimic natural cellular systems.

\subsection{Model Membranes to Characterize Proteins and Protein In- teractions}

Many proteins are embedded in or at least temporarily linked to biological membranes.

To study protein-lipid or protein-protein interactions or functionally characterize single proteins (i.e. ion channels) various types of artificial membrane systems have been established. They serve as versatile platforms to characterize proteins or investigate their interactions in an environment that resembles cellular structures. As for transmembrane proteins, the reconstitution in membranes is even mandatory to obtain their full functionality and prevent protein denaturation.

As a matter of fact, each system offers certain advantages, depending on the aspects that are wished to be addressed. A selection of possible model membrane systems is depicted in Fig. 1.3. 
Vesicles

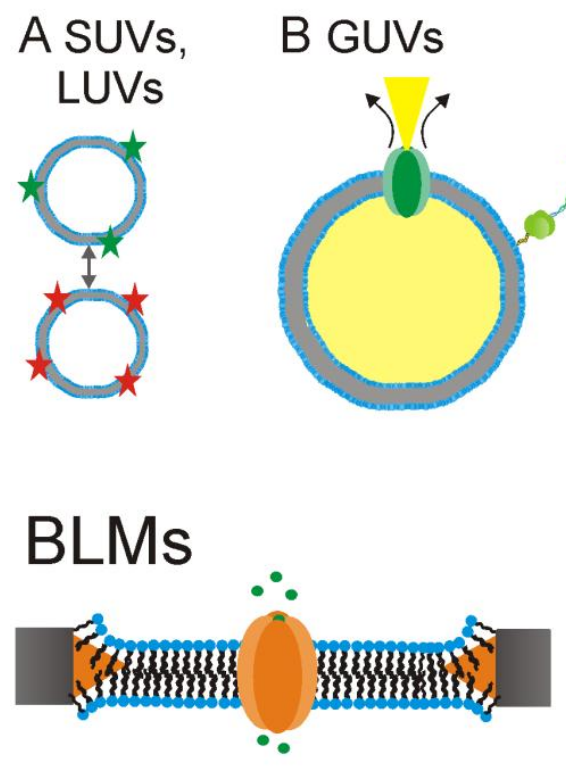

\section{PSMs}

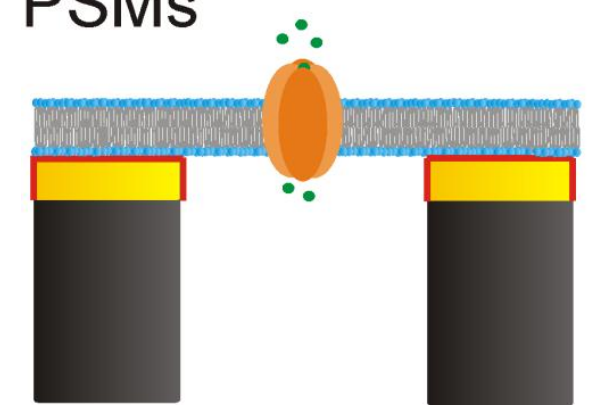

\section{SSMs}
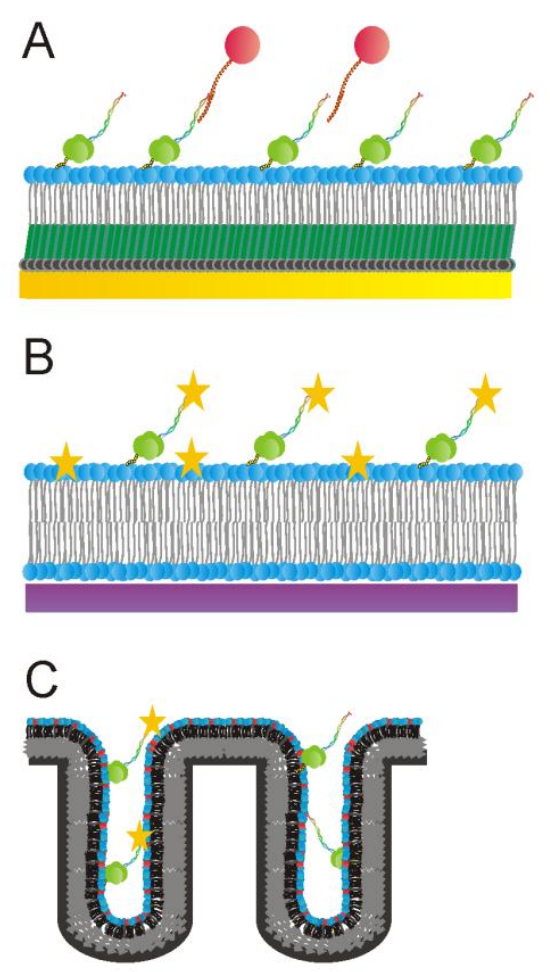

\section{Tethered SSMs}

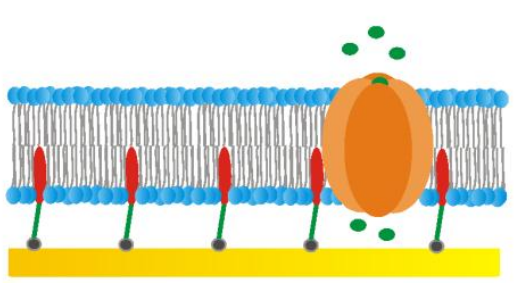

Fig. 1.3 Schematic drawing of selected model membranes systems. Depicted are small, large and giant unilamellar vesicles (SUVs, LUVs and GUVs), solid supported membranes (SSMs) that are either gold supported and hybrid membranes (self assembled monolayer and lipid monolayer) (A), silicon supported bilayers (B) or hybrid membranes on porous material (C), black lipid membranes (BLMs), pore spanning membranes (PSMs) and tethered solid supported membranes. Stars represent fluorescent labels of proteins or lipids.

Small or large unilamellar vesicles (SUVs or LUVs) for instance can be used to study fusion processes or other fluorescence coupled phenomena. Van den Bogaart et al. investigated the fusogenity of SNARE protein containing vesicles by means of fluorescence resonance energy transfer (FRET) experiments. ${ }^{26}$ Actin networks have been reconstituted in giant unilamellar vesicles (GUVs) by Pontani et al. to mimic cellular 
structures. Tamba et al. demonstrated that magainin 2 permeabelizes the membrane of GUVs by showing the release of a fluorescence dye that was entrapped inside the GUVs. ${ }^{27}$ In general, GUVs with a size of several micrometers properly resemble eukaryotic cells in terms of their dimensions and are suitable for optical experiments such as fluorescence microscopy. Hence, numerous experiments have been conducted that deal with the influence of protein binding on lipid and membrane organization. ${ }^{28,29}$

Solid supported membranes (SSMs) can be either hybrid, gold supported membranes composed of a self assembled monolayer and a lipid monolayer or on planar lipid bilayers with a silicon, glass or mica solid support. A three dimensional surface can be generated using porous anodic aluminum oxide (AAO) pores as a solid support. Lazzara et al. developed a biosensor assay based on porous AAO substrates. ${ }^{30}$ By means of optical waveguide spectroscopy (OWS) and confocal laser scanning microscopy (CLSM), they investigated the adsorption of protein on differently functionalized AAO films. ${ }^{31,32}$ Owing to the three dimensional structure of porous AAO films, they offer a good mimic to cragged cellular structures such as membrane ruffles and microvilli.

In contrast to those membranes that lack a solid support, SSMs do not offer an enclosed inner compartment and have a reduced lateral mobility. However, they are invaluable tools to investigate adsorption processes of proteins in a time resolved manner, as the solid support can serve as a transducer surface of optical and acoustical biosensors. ${ }^{33-35}$ Also, the smooth surface of lipid bilayers formed on silicon or mica is suitable for imaging proteins by means of atomic force microscopy (AFM) giving insight into the proteins dimension and the lateral protein organization. ${ }^{36,37}$ Tethered lipid bilayers posses an aqueous phase between the membrane and the solid support, as the applied thiol lipids serve as spacer that decouples the membrane from the solid support. ${ }^{38}$ Thus, the investigation of membrane transport processes by means of electrochemical techniques such as impedance spectroscopy is more feasible compared to solid supported hybrid membranes. Although both tethered lipid bilayers and unilamellar vesicles enclose an inner cavity, it is not possible to access this compartment in order to vary its contents or, in case of the vesicles, electrochemically address this compartment. Black lipid 
membranes (BLMs) allow to separately control and address both compartments. BLMs have been used for over 50 years to reconstitute and characterize channel proteins such as the bacterial outer membrane channels. ${ }^{39}$ Since they span an area with a diameter up to the lower millimeter range (100 $\mu \mathrm{m}-2 \mathrm{~mm})$, their long term stability is strongly decreased compared to SSMs. ${ }^{40}$ Pore spanning membranes (PSMs) on the contrary only have a free standing area with diameters ranging from several nanometers up to a few micrometers. Hence, they combine the advantages of SSMs and BLMs, as their long term stability is significantly increased compared to BLMs and an accessibility and a control of both aqueous compartments is provided. Besides the feasibility of electrochemical characterizing transport processes through channel proteins, PSMs can also be mechanically addressed using the atomic force microscope (AFM). ${ }^{41-43}$ The influence of embedded or membrane linked proteins on the membrane mechanics can thus be elucidated. 


\subsection{References}

[1] Jones, S., and Thornton, J. M. (1996) Principles of protein-protein interactions. Proc. Natl. Acad. Sci. U.S.A 93, 13-20.

[2] Phizicky, E. M., and Fields, S. (1995) Protein-protein interactions: methods for de tection and analysis. Microbiol. Rev. 59, 94-123.

[3] Bock, J. R., and Gough, D. A. (2001) Predicting protein--protein interactions from primary structure. Bioinformatics 17, 455-460.

[4] Nooren, I. M., and Thornton, J. M. (2003) Diversity of protein-protein interact tions. EMBO J. 22, 3486-3492.

[5] Las Rivas, J. de, and Fontanillo, C. (2010) Protein-protein interactions essentials: key concepts to building and analyzing interactome networks. PLOS Comput. Biol. 6, e1000807.

[6] Salwinski, L. (2004) The Database of Interacting Proteins: 2004 update. Nucleic Acids Res. 32, 449D-451.

[7] Voet, D., and Voet, J. G. (2011) Biochemistry. 4th ed., John Wiley \& Sons, Hoboken, NJ.

[8] Chothia, C., and Janin, J. (1975) Principles of protein-protein recognition. Nature 256, 705-708.

[9] Ma, B., Elkayam, T., Wolfson, H., and Nussinov, R. (2003) Protein-protein interactions: structurally conserved residues distinguish between binding sites and exposed protein surfaces. Proc. Natl. Acad. Sci. U.S.A 100, 5772-5777.

[10] Moreira, I. S., Fernandes, P. A., and Ramos, M. J. (2007) Hot spots--a review of the protein-protein interface determinant amino-acid residues. Proteins 68, 803812. 
[11] Schreiber, G. (2002) Kinetic studies of protein-protein interactions. Curr. Opin. Struct. Biol. 12, 41-47.

[12] Roos, H., Karlsson, R., Nilshans, H., and Persson, A. (1998) Thermodynamic analysis of protein interactions with biosensor technology. J. Mol. Recognit. 11, 204-210.

[13] Myszka, D. G. (1997) Kinetic analysis of macromolecular interactions using sur face plasmon resonance biosensors. Curr. Opin. Biotechnol. 8, 50-57.

[14] Piehler, J. (2005) New methodologies for measuring protein interactions in vivo and in vitro. Curr. Opin. Struct. Biol. 15, 4-14.

[15] Tang, X., and Bruce, J. E. (2009) Chemical Cross-Linking for Protein-Protein Interaction Studies, Method. Moll Biol.492 283-293.

[16] Ibraheem, A., and Campbell, R. E. (2010) Designs and applications of fluorescent protein-based biosensors. Curr. Opin. Chem. Biol. 14, 30-36.

[17] Langowski, J. (2008) Protein-protein interactions determined by fluorescence correlation spectroscopy. Method. Cell Biol. 85, 471-484.

[18] Lakey, J. H., and Raggett, E. M. (1998) Measuring protein-protein interactions. Curr. Opin. Struct. Biol. 8, 119-123.

[19] Cooper, M. A. (2003) Label-free screening of bio-molecular interactions. Analytical and Bioanalytical Chemistry 377, 834-842.

[20] Haake, H.-M., Schütz, A., and Gauglitz, G. (2000) Label-free detection of biomolecular interaction by optical sensors. Fresen. J. Anal. Chem. 366, 576-585.

[21] Szabo, A., Stolz, L., and Granzow, R. (1995) Surface plasmon resonance and its use in biomolecular interaction analysis (BIA). Curr. Opin. Struct. Biol. 5, 699-705.

[22] Rich, R. L., and Myszka, D. G. (2008) Survey of the year 2007 commercial optical biosensor literature. J. Mol. Recognit. 21, 355-400. 
[23] Cheng, C. I., Chang, Y.-P., and Chu, Y.-H. (2012) Biomolecular interactions and tools for their recognition: focus on the quartz crystal microbalance and its diverse surface chemistries and applications. Chem Soc Rev 41, 1947-1971.

[24] Yang, Y., Wang, H., and Erie, D. A. (2003) Quantitative characterization of biomolecular assemblies and interactions using atomic force microscopy. Methods 29, 175-187.

[25] Nevo, R., Brumfeld, V., Elbaum, M., Hinterdorfer, P., and Reich, Z. (2004) Direct discrimination between models of protein activation by single-molecule force measurements. Biophys. J. 87, 2630-2634.

[26] van den Bogaart, G., Holt, M. G., Bunt, G., Riedel, D., Wouters, F. S., and Jahn, R. (2010) One SNARE complex is sufficient for membrane fusion. Nat Struct Mol Biol 17, 358-364.

[27] Tamba, Y., and Yamazaki, M. (2005) Single Giant Unilamellar Vesicle Method Reveals Effect of Antimicrobial Peptide Magainin 2 on Membrane Permeability. Biochemistry 44, 15823-15833.

[28] Carvalho, K., Ramos, L., Roy, C., and Picart, C. (2008) Giant Unilamellar Vesicles Containing Phosphatidylinositol(4,5)bisphosphate: Characterization and Functionality. Biophys. J. 95, 4348-4360.

[29] Römer, W., Berland, L., Chambon, V., Gaus, K., Windschiegl, B., Tenza, D., Aly, M. R. E., Fraisier, V., Florent, J.-C., Perrais, D., Lamaze, C., Raposo, G., Steinem, C., Sens, P., Bassereau, P., and Johannes, L. (2007) Shiga toxin induces tubular membrane invaginations for its uptake into cells. Nature 450, 670-675.

[30] Lazzara, T. D., Mey, I., Steinem, C., and Janshoff, A. (2011) Benefits and Limitations of Porous Substrates as Biosensors for Protein Adsorption. Anal. Chem 83, $5624-5630$.

[31] Lazzara, T. D., Behn, D., Kliesch, T.-T., Janshoff, A., and Steinem, C. (2012) Phospholipids as an alternative to direct covalent coupling: surface functionalization 
of nanoporous alumina for protein recognition and purification. J. Colloid. Interf. Sci. 366, 57-63.

[32] Lazzara, T. D., Kliesch, T.-T., Janshoff, A., and Steinem, C. (2011) Orthogonal functionalization of nanoporous substrates: control of 3D surface functionality. ACS Appl Mater Interfaces 3, 1068-1076.

[33] Mozsolits, H., and Aguilar, M.-I. (2002) Surface plasmon resonance spectroscopy: An emerging tool for the study of peptide-membrane interactions. Biopolymers 66, 3-18.

[34] MacKenzie, C. R., Hirama, T., Lee, K. K., Altman, E., and Young, N. M. (1997) Quantitative analysis of bacterial toxin affinity and specificity for glycolipid receptors by surface plasmon resonance. J. Biol. Chem. 272, 5533-5538.

[35] Janshoff, A., and Steinem, C. (2005) Label-free detection of protein-ligand interacttions by the quartz crystal microbalance. Method. Mol. Biol. 305, 47-64.

[36] Yang, J., Tamm, L. K., Tillack, T. W., and Shao, Z. (1993) New approach for atomic force microscopy of membrane proteins. The imaging of cholera toxin. $J$. Mol. Biol. 229, 286-290.

[37] Herrig, A., Janke, M., Austermann, J., Gerke, V., Janshoff, A., and Steinem, C. (2006) Cooperative Adsorption of Ezrin on PIP 2 -Containing Membranes. Bio chemistry 45, 13025-13034.

[38] Giess, F., Friedrich, M. G., Heberle, J., Naumann, R. L., and Knoll, W. (2004) The Protein-Tethered Lipid Bilayer: A Novel Mimic of the Biological Membrane. Biophys. J. 87, 3213-3220.

[39] van Gelder, P., Dumas, F., and Winterhalter, M. (2000) Understanding the func tion of bacterial outer membrane channels by reconstitution into black lipid membranes. Biophys. Chem. 85, 153-167.

[40] Winterhalter, M. (2000) Black lipid membranes. Curr. Opin. Colloid In. 5, 250-255. 
[41] Mey, I., Stephan, M., Schmitt, E. K., Müller, M. M., Ben Amar, M., Steinem, C., and Janshoff, A. (2009) Local membrane mechanics of pore-spanning bilayers. J. Am. Chem. Soc. 131, 7031-7039.

[42] Steltenkamp, S., Müller, M. M., Deserno, M., Hennesthal, C., Steinem, C., and Janshoff, A. (2006) Mechanical properties of pore-spanning lipid bilayers probed by atomic force microscopy. Biophys. J. 91, 217-226.

[43] Kocun, M., Lazzara, T. D., Steinem, C., and Janshoff, A. (2011) Preparation of solvent-free, pore-spanning lipid bilayers: modeling the low tension of plasma membranes. Langmuir 27, 7672-7680. 


\section{Scope of the Thesis}

Protein interactions that take place within cells often occur at membrane interfaces. Apart from investigating protein interactions in vivo, it has been of widespread scientific interest to study these interactions in vitro, with the benefit of being able to control all variable parameters like protein and lipid composition as well as surface geometries.

The aim of this work is to investigate specific interactions of membrane associated proteins in vitro, utilizing artificial membranes that mimic the corresponding cellular membrane structure. Owing to the uniqueness of cellular membranes as surfaces that mediate protein interactions, a great effort is made to adapt the character of each artificial membrane to the needs of the proteins under investigation.

First, the interactions between $C$-terminal polycystin-2 (cPC2) and some of its putative interaction partners are investigated. The full length transmembrane protein polycystin-2, (PC2) regulates cellular proliferation and differentiation as part of an interaction network. Here, a biosensor assay based on solid supported membranes (SSMs) is developed that allows to quantify these protein-protein interactions by means of the quartz crystal microbalance (QCM) and surface plasmon resonance (SPR) technique. The benefit of this assay is that it enables one to study the interaction of membrane associated proteins, providing their natural environment, by coupling one interaction partner to the membrane surface (chapter 4 ).

This assay is then applied to quantify the interaction of cPC2 with the C-terminal polycystin-1 (cPC1) as a function of $\mathrm{Ca}^{2+}$ concentration (chapter 5). Inside cellular systems, the interaction of the full length proteins takes place at the plasma membrane and is supposably responsible for regulating cellular development. Since several groups reported $\mathrm{Ca}^{2+}$ induced structural changes of $\mathrm{cPC} 2$ or protein oligomerization, it is of high 
interest to elucidate, whether these changes have an impact on the cPC2-cPC1 interaction.

The influence of cPC2 pseudophosphorylation at Ser ${ }^{812}$ on the interaction with PIGEA14 is the following matter of investigation (chapter 6). Extracting dissociation constants and kinetic rate constants of the interaction, it is aimed to test a model proposed earlier, which hypothesizes that a phosphorylation at Ser ${ }^{812}$ would diminish the interaction between PC2 and PIGEA14 in the cell and thus hamper the retrograde PC2 transport to the plasma membrane.

The interaction of filamentous actin (F-actin) and ezrin mainly takes place inside the mi-crovilli of polarized epithelial cells, providing the stability and dynamics of these finger-like cellular protrusions. SSMs lining the interior of optically transparent porous anodic aluminum oxide (AAO) films are used to mimic the cellular microvilli in order to study adsorption processes that occur atop and inside these porous structures. It is aimed to control the adsorption process of F-actin at the AAO membrane surface by means of opti-cal waveguide spectroscopy (OWS) and confocal laser scanning microscopy (CLSM) us-ing different surface funtionalization strategies (chapter 7).

The impact of a membrane attached F-actin network on the tension and viscoelastic properties of pore spanning membranes (PSMs) is going to be studied by means of atom-ic force microscopy (AFM, chapter 8). In eukaryotic cells, numerous actin binding proteins (ABPs) regulate the cortical F-actin network, thus governing the mechanical properties of the cells. The great benefit of this in vitro set up based on PSM, compared to directly measuring the cellular mechanics, is that the impact of each actin binding protein $(\mathrm{ABP})$ on the membrane mechanics can be addressed and studied independently from one another. 


\subsection{Experimental Methods}

\subsubsection{Preparation of Nanoporous Anodic Aluminum Oxide Substrates}

As a nonporous barrier oxide, anodic aluminum oxide (AAO) is employed in manifold applications such as aluminum protection from environmental damage and catalysis since the early $20^{\text {th }}$ century. ${ }^{1}$ Highly ordered nanoporous AAO materials have been used in the last 40 years in the fields of sensing, separation and more recenlty as a support for pore spanning membranes (PSMs). ${ }^{2,3}$ Basically, growth of AAO is induced by electrochemical anodization that, in the presence of a proper electrolyte, causes the conversion of $\mathrm{Al}$ metal to aluminum oxide (eq. 3.1 and eq. 3.2).

Anode: $2 \mathrm{Al}^{3+}+3 \mathrm{H}_{2} \mathrm{O} \longrightarrow \mathrm{Al}_{2} \mathrm{O}_{3}+6 \mathrm{H}^{+}+6 \mathrm{e}^{-}$

eq. 3.1

Cathode: $2 \mathrm{H}^{+}+2 \mathrm{e}^{-}$

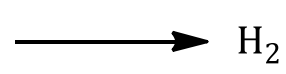

eq. 3.2

While barrier oxide forms in a solution with $\mathrm{pH}<5$ or in acids that only poorly dissolve the alumina, porous $\mathrm{AAO}$ is formed in the presence of acidic solutions that moderately dissolve $\mathrm{Al}_{2} \mathrm{O}_{3}$ such as $\mathrm{H}_{2} \mathrm{SO}_{4}, \mathrm{H}_{3} \mathrm{PO}_{4}$ or $\mathrm{H}_{2} \mathrm{C}_{2} \mathrm{O}_{4} .{ }^{4}$ Concentrated solutions of $\mathrm{H}_{2} \mathrm{SO}_{4}$ and $\mathrm{H}_{3} \mathrm{PO}_{4}$ strongly dissolve the formed alumina and are therefore used for electropolishing, a process to reduce the surface roughness of the aluminum metal plates.

Porous AAO membranes are composed of a thin barrier layer $(10-100 \mathrm{~nm})$ neighboring the aluminum metal. The porous region can extent up to several micrometers depend- 
ing on the anodization time and the applied voltage. In 1998 Jessensky et al. found a higher volume expansion of the aluminum during oxidation in $20 \% \mathrm{H}_{2} \mathrm{SO}_{4}$, if a higher voltage was applied. ${ }^{5}$ Besides this, the lattice constant $\left(\lambda_{\mathrm{c}-\mathrm{c}}\right)$ of the honeycomb like structured pore array is proportional to the applied voltage at a given electrolyte. Li et al. showed that the lattice constant of a hexagonally ordered pore array can be tuned by varying the electrolyte and the applied voltages from 19-160 V. AAO pores with lattice constants between $50-420 \mathrm{~nm}$ in the following order obtained were produced: $\lambda_{\mathrm{c}}$ -

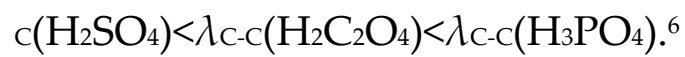

In this work, a $0.3 \mathrm{M}$ oxalic acid solution and $40 \mathrm{~V}$ were applied at $1{ }^{\circ} \mathrm{C}$ to fabricate hexagonally ordered AAO pores with $\lambda_{\mathrm{c}-\mathrm{C}}=100 \mathrm{~nm}$ with a resulting AAO thickness growth of $2.5 \mu \mathrm{m} / \mathrm{h}^{7}$ A schematic drawing of nanoporous AAO and scanning electron microscopy (SEM) micrographs are shown in Fig. 3.1.
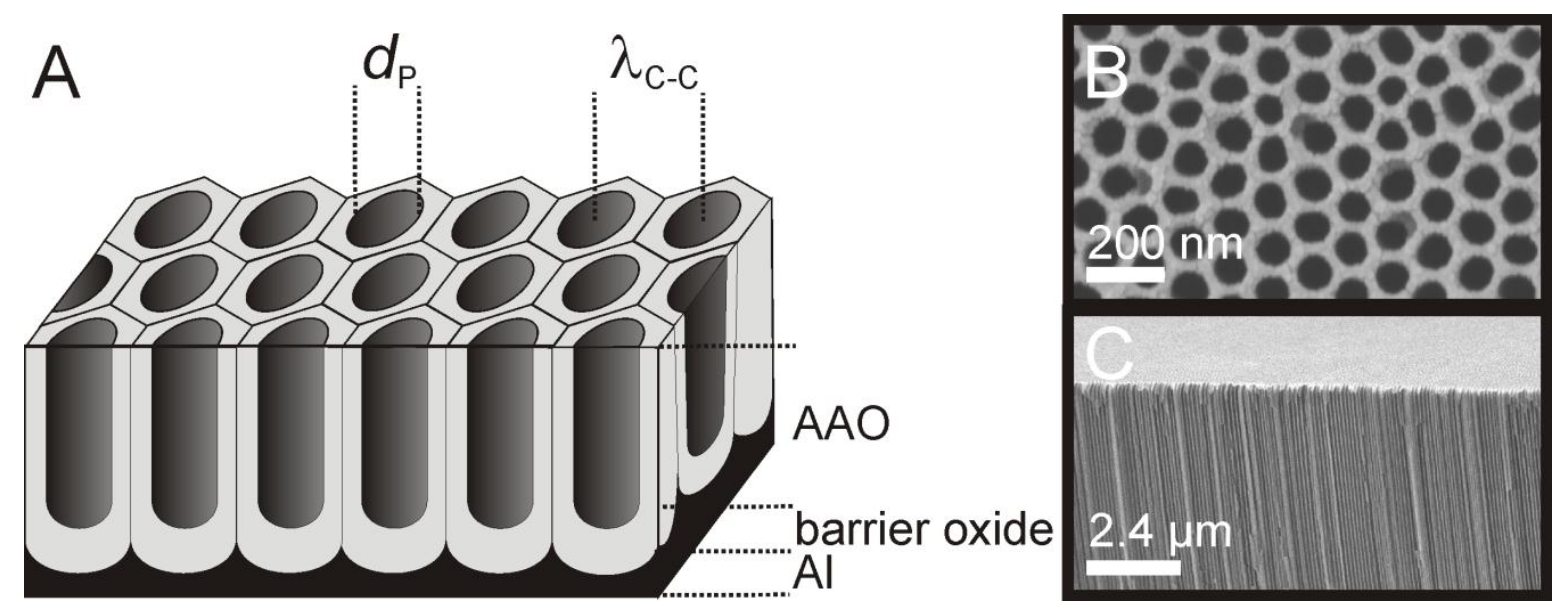

Fig. 3.1 (A) Schematic three dimensional drawing of a highly ordered AAO substrate fabricated by electrochemical anodization of $\mathrm{Al}$ metal plates. The anodization condition (electrolyte, voltage, temperature, time) controls the shown dimensions such as the lattice constant $\left(\lambda_{\mathrm{c}-\mathrm{c}}\right)$, the barrier oxide thickness and the AAO thickness. Size of the pore diameter $(d \mathrm{P})$ can be tuned via pore widening in $5 \% \mathrm{H}_{3} \mathrm{PO}_{4}$. (B) SEM micrograph of the top view and side view $(\mathbf{C})$ of highly ordered AAO pores anodized in $0.3 \mathrm{M}$ oxalic acid solution at $40 \mathrm{~V}, 1^{\circ} \mathrm{C}$ for $2.5 \mathrm{~h}$. The AAO was pore widened in $5 \% \mathrm{H}_{3} \mathrm{PO}_{4}$ for $60 \mathrm{~min}\left(d_{\mathrm{P}} \approx 65 \mathrm{~nm}\right)$ ).

In 1970 O'Sullivan proposed a model that describes the process of self regulated pore growth. ${ }^{8}$ Although this model has been refined over the past decades, pore growth is 
basically governed by a difference in electrical field distribution at the pore tops and bottoms. The alumina expansion during pore growth causes a mechanical repulsive stress that provides the actual driving force of highly ordered honeycomb shaped pores. More explicitly, growth of nanoporous aluminum oxide during electrochemical anodization can be classified into four steps (Fig. 3.2).
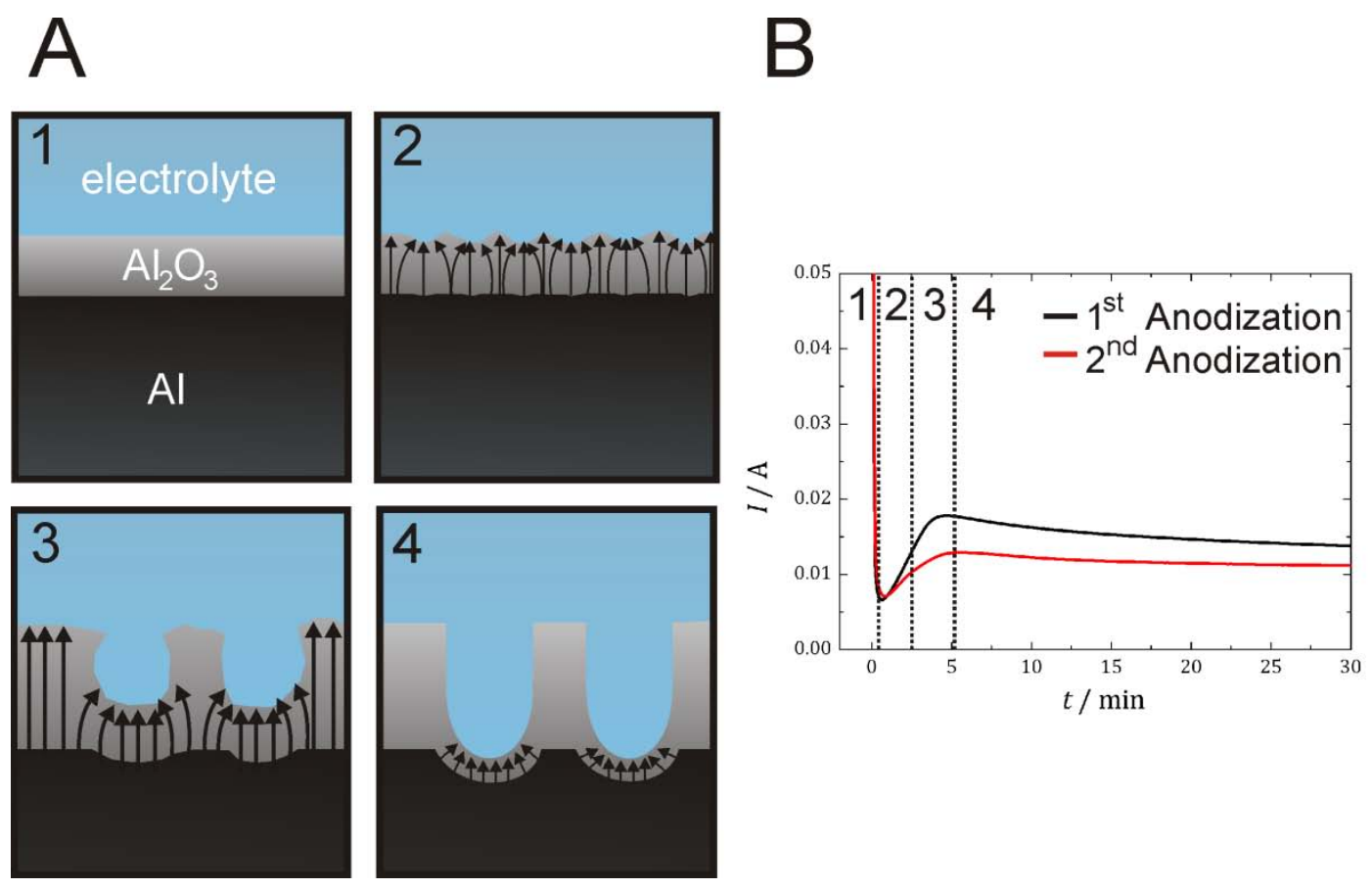

Fig. 3.2 (A) Schematic drawing of the four different steps of self regulated alumina growth leading to the formation of honeycomb structured highly ordered porous AAO. (B) Time course of the current shift during the first anodization (black) of electropolished aluminium and second anodization (red) of the pre-textured aluminum surface (first 30 minutes are shown). The respective steps (1-4) are marked inside the graph.

Step 1 refers to a drop of current flow within the first minutes of anodization indicating the formation of a thin aluminum oxide barrier layer. The current then starts to rise slowly in step 2 reflecting the development of surface perturbations. This leads to local electrical field distributions that cause an increased dissolution of $\mathrm{Al}^{3+}$ at these cavities. In step 3 disordered pores form. The current still rises until a maximum is reached corresponding to a high rate of $\mathrm{Al}$ oxidation and $\mathrm{Al}^{3+}$ dissolution inside the pores and thus 
an increased current flow. After the whole surface is covered with disordered pores the process of pore growth becomes self regulating due to repulsive forces between the walls (step 4). A state of equilibrium is reached, at which the pores grow with a constant rate characterized by a slight decrease of current flow over the whole anodization time.

To obtain highly ordered porous AAO substrates Masuda et al. developed a two step process including a first anodization as a pre-texturing step, the dissolution of the less ordered porous AAO that leaves a pre-textured, highly ordered aluminum bottom and a second anodization that results in the formation of highly ordered alumina pores. ${ }^{9}$ The two anodization steps are depicted in Fig. 3.2 B. Remarkably, the two curves show different characteristics such as a slower current increase, no overshoot phenomenon and a higher minimum in case of the second anodization. ${ }^{4}$

Besides improving the degree of order of the AAO by two step anodization, it has been reported previously that the annealing of the aluminum foil prior to further treatment improves the pore ordering significantly. ${ }^{10}$ This can be explained with a grain growth and thus a reduction of grain boundaries due to annealing of the Al metal. Within these grains the face centered cubic cell that is formed by aluminum atoms are perfectly arranged and oriented. A reduction of grain boundaries leads to a macroscopically increased ordering and additionally reduces the surface roughness of the aluminum plates. ${ }^{11}$ In this work, the two step anodization process developed by Masuda et al. was adapted with annealed Al metal plates and will be described in detail in the following sections.

\section{Electrochemical Polishing of Aluminum Plates}

High purity aluminum plates (99.999\%) with a square size of $2.5 \times 2.5 \mathrm{~cm}$ from Goodfellow (Bad Nauheim, Germany) were annealed overnight at $500{ }^{\circ} \mathrm{C}$ and slowly cooled to room temperature to allow the formation of highly ordered pores within macroscopic grains. 
Electrochemichal polishing of the alumina plates was performed in a four-hole Teflon chamber applying $24 \mathrm{~V}$ at $70{ }^{\circ} \mathrm{C}$ for $15 \mathrm{~min}$ in a strong acid solution $\left(250 \mathrm{~g} \mathrm{H}_{2} \mathrm{O}, 250 \mathrm{~g}\right.$ $85 \% \mathrm{H}_{3} \mathrm{PO}_{4}$ and $250 \mathrm{~g}$ conc. $\mathrm{H}_{2} \mathrm{SO}_{4}$ ) that immediately dissolves the formed barrier oxide and thus leaves a perfectly smooth surface. Before the four plates were placed inside the Teflon chamber, the copper bottom was treated with abrasive paper. It was taken care of a tight sealing, because any leakage during the polishing steps would affect the quality of the aluminum plates. After the first polishing step, the solution was exchanged and the 4 plates were re-polished until they were as smooth as possible indicated by a mirror-like reflectivity. The aluminum plates were subsequently rinsed with MilliQ water and ethanol p.a. and immersed in a $5 \% \mathrm{H}_{3} \mathrm{PO}_{4}$ solution for approximately one $\mathrm{h}$ to remove the film of disordered $\mathrm{Al}_{2} \mathrm{O}_{3}$ that covers the polished $\mathrm{Al}$ metal surface. It was of major importance to rinse the plates with ethanol p.a., before re-immersion with $\mathrm{H}_{3} \mathrm{PO}_{4}$ solution to reduce the surface tension and facilitate the penetration of the acid solution inside the perturbations. When all traces of $\mathrm{Al}_{2} \mathrm{O}_{3}$ in terms of a white or a dim-violet film were vanished from the aluminum plates after drying them in a stream of nitrogen, the second anodization step could be performed.

Surface roughness can be reduced from $5 \mu \mathrm{m}$ to $20-30 \mathrm{~nm}$ during the process of electrochemical polishing. ${ }^{5}$ A SEM micrograph (Fig. 3.3) shows a non-polished, annealed aluminum foil (A) and a polished aluminum plate (B). 

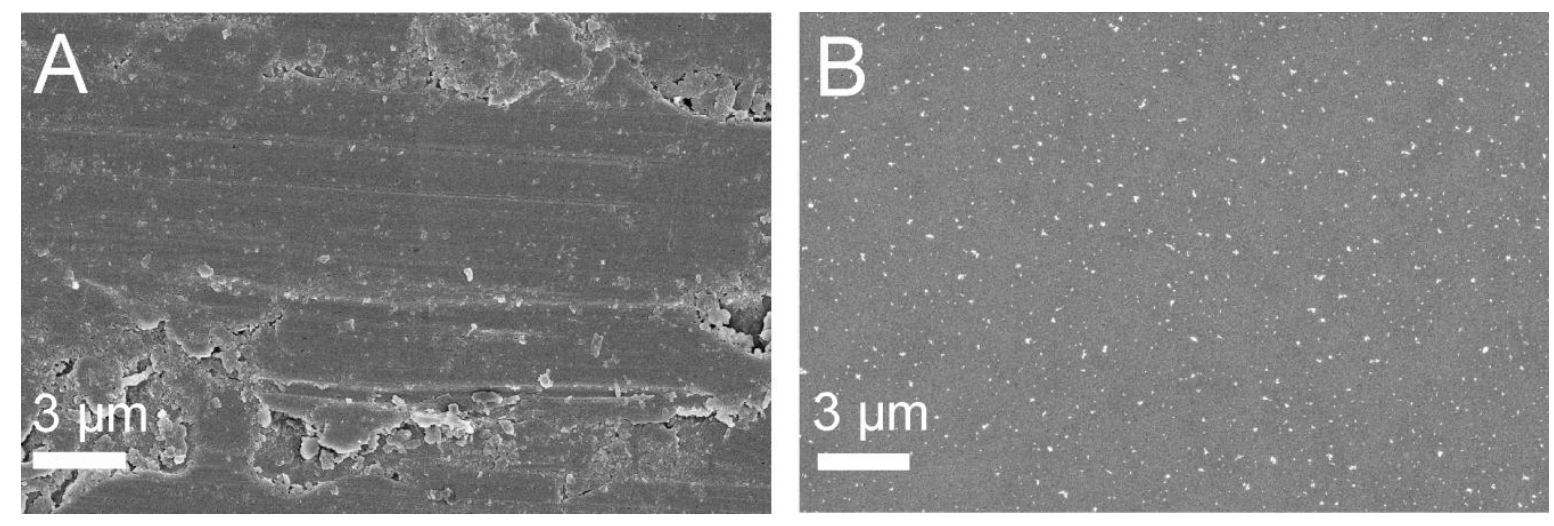

Fig. 3.3 SEM micrograph of an unpolished, annealed aluminium plate (A) and an aluminum plate after electrochemical polishing (B).

\section{Fabrication of Ordered Porous Anodic Aluminum Oxide Substrates}

After electrochemical polishing of the aluminum metal plates, they were transferred to a polyvinylchloride four-hole chamber that provided a tighter seal and thus prevents leakage during the anodization process. The chamber was placed on a cooling block that was equipped with a Peltier element for temperature control with $T=1.5^{\circ} \mathrm{C}$. Precooled $0.3 \mathrm{M}$ oxalic acid solution was poured inside the chamber to immerse the Al plates. A stirrer was attached to the aperture and the system was left for 10-30 min to reach a temperature of $1.5^{\circ} \mathrm{C}$. The first anonization was then performed at $40 \mathrm{~V}$ for $2.5 \mathrm{~h}$ to pre-texture the aluminum surface. The AAO plates were rinsed with MilliQ water and ethanol p.a., before the formed, less ordered pores (Fig. $3.4 \mathrm{~A}$ ) were dissolved in a $5 \% \mathrm{H}_{3} \mathrm{PO}_{4}$ solution for approximately 2-3 $\mathrm{h}$. The dissolution of the AAO surface was controlled as described above. A SEM micrograph of the pre-textured aluminum bottom is shown in Fig. 3.4 B. The second anodization was carried out as the first one, but with variable anodization times from 1.5-2.5 h, so that either $3.5 \mu \mathrm{m}$ thick porous AAO substrates for optical waveguide spectroscopy (OWS) experiments or AAOs with a $7 \mu \mathrm{m}$ thickness for confocal laser scanning microscopy (CLSM) experiments were obtained. A SEM micrograph shows the highly ordered pores as the result of the second anodization step (Fig. 3.4 C). 

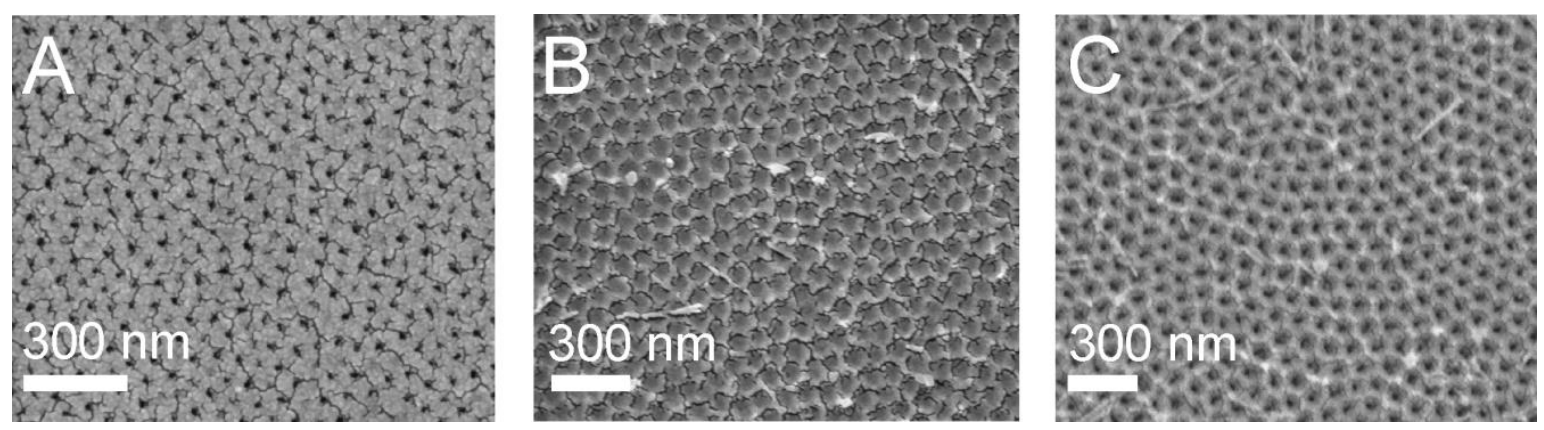

Fig. 3.4 SEM micrograph of a porous AAO film after the first anodization(A), the pre-textured aluminum pore bottom after dissolution of the AAO $(\mathbf{B})$ and the highly ordered AAO pores after the second anodization step (C).

\section{Further Processing of Porous Anodic Aluminum Oxide Substrates}

First, the AAO plates were prepared to remove the remaining aluminum with acidic $\mathrm{CuCl}_{2}$ solution (etching solution). In order to do so, each plates was surrounded with double sided tape from Tesa (Hamburg, Germany) and glued on a glass slide with the $\mathrm{AAO}$ facing the glass. On the back side of the aluminum plates, lines were drawn to delimit the AAO-Al metal boundary. The periphery was then covered with a two component epoxy glue from UHU (Brühl, Germany) to produce a seal that protects the $\mathrm{AAO}$ from the $\mathrm{CuCl}_{2}$-solution during the etching process. After the epoxy was cured (2-3 $\mathrm{h}$ ), the substrates were immersed in etching solution $\left(8.5 \mathrm{~g} \mathrm{CuCl}_{2}, 250 \mathrm{~g} \mathrm{H}_{2} \mathrm{O}, 250 \mathrm{~g}\right.$ conc. $\mathrm{HCl}$ ) that contained a hint of ethanol p.a. to reduce surface tension. During the etching process $\mathrm{Cu}^{2+}$ is reduced to $\mathrm{Cu}$ metal, while $\mathrm{Al}$ metal is oxidized to $\mathrm{Al}^{3+}$. $\mathrm{Cu}$ that was formed on top of the Al surface was immediately rinsed away with a glass pipette. After transparent spots of AAO became visible, the substrate was transferred to a petri dish filled with etching solution that was cooled on ice. Dissolution of Al metal took place as long as there were still traces of aluminum left on the AAO bottom surface. After the etching process the substrates were immersed in MilliQ water containing a hint of ethanol, rinsed thoroughly with MilliQ water and ethanol p.a. and carefully dried under a stream of nitrogen in a way that all drying stains were diminished on the surface. 
For CLSM experiments, the $7 \mu \mathrm{m}$ thick AAO substrates were broken into small pieces with extra sharp tweezers and glued on a glass plate with the pores facing upwards. To prepare the glue two drops of UV-curable optical adhesive (NOA $83 \mathrm{H})$ were dissolved in $1.5 \mathrm{~mL}$ of THF from Norland Products (Cranbury, USA). The glass plate had been cleaned via ultrasonication in $2 \%$ Hellmanex $^{\circledR}$ solution and MilliQ water, 15 min at room temperature and cut into a desired size. The glue was cured for at least $30 \mathrm{~min}$ under an UV-lamp.

For OWS experiments the AAO bottom side had to be covered with a 20-30 nm coupling layer of gold. To prevent any damages caused by the vacuum during evaporation, a small hole was cut into the glue between the $\mathrm{Al}$ plate and the glass slide before evaporation. The substrate was then placed inside a Bal-Tec MCS610 thermal evaporation unit equipped with a QSG060 thickness monitor and gold was evaporated at 5·10${ }^{9}$ bar while the substrates were rotating. After the substrates had been coated with gold, they were broken into pieces with a diameter of approximately $0.5 \mathrm{~cm}$ and glued (UVcurable glue dissolved in THF as described above) to a LaSFN9 high refractive index glass slide with the gold facing the glass and the porous side facing upwards.

The AAO pores could then be widened to obtain pores with a diameter from $25 \mathrm{~nm}$ to $75 \mathrm{~nm}$. According to eq. 3.3 the pore widening process is linearly proportional to the time it is immersed in a $5 \%(v / v)$ solution of conc. $\mathrm{H}_{3} \mathrm{PO}_{4}$ at $20^{\circ} \mathrm{C}$ under constant stirring. ${ }^{7}$

$$
d_{\text {Pore }}=0.75 \cdot t+25 \mathrm{~nm}
$$

eq. 3.3

In this work, porous AAO substrates with diameters of 25, 35, 45, and $65 \mathrm{~nm}$ were prepared to investigate the influence of pore dimensions on the penetration ability of filamentous actin (see chapter 7, page 195). It is important to note that, if any modifications 
were performed in a liquid environment (spreading, F-actin binding), that AAO substrate was first rinsed thoroughly with ethanol p.a. to allow the penetration of buffer in the pore interior.

\subsubsection{Functionalization Strategies}

Self assembled monolayers (SAMs) have gained significant attraction in corrosion inhibition, biosensing and as platform for chemical surface coupled reactions. ${ }^{12,13}$ The most popular representatives of SAMs are thiols on gold ${ }^{13,14}$ and trichloro- or tri-ethoxy silanes on silicon or aluminum oxide surfaces. ${ }^{15}$ Both systems obtain certain advantages. While SAMs on gold are easy to prepare with high reproducibility, SAMs composed of silanes on $\mathrm{SiO}_{2}$ exhibit higher physical and chemical stability once adsorbed on the surface. However, the assembly of $\mathrm{SAM}$ on $\mathrm{SiO}_{2}$ seems to be more complex compared to thiols on gold. Also, most silanes used for surface functionalization react with traces of water, so careful handling during the preparation is mandatory. ${ }^{12}$ Basically, SAM compounds can be divided in three parts: the head group, alkyl chain and terminal group that often carries a chemical functionality. Preparation of SAMs can either be carried out in liquid- or vapor-phase. Kinetics and mechanism of SAM formation have been well investigated in the past. ${ }^{14}$ The rate of formation is dependent on compound concentration, temperature, solvent and chain length. The adsorption process of thiols on gold surfaces can be characterized by two phases: the initial phase takes place within minutes and corresponds to a fast adsorption of thiols that leads to a final surface coverage of $80-90 \% .{ }^{14}$ The next phase includes processes such as lateral diffusion, additional adsorption and enhanced packing to obtain a surface coverage of up to $99 \%$. The densely packed, pseudocrystalline assemblies are tilted $20-30^{\circ}$ from the surface normal (Fig. 3.5). 


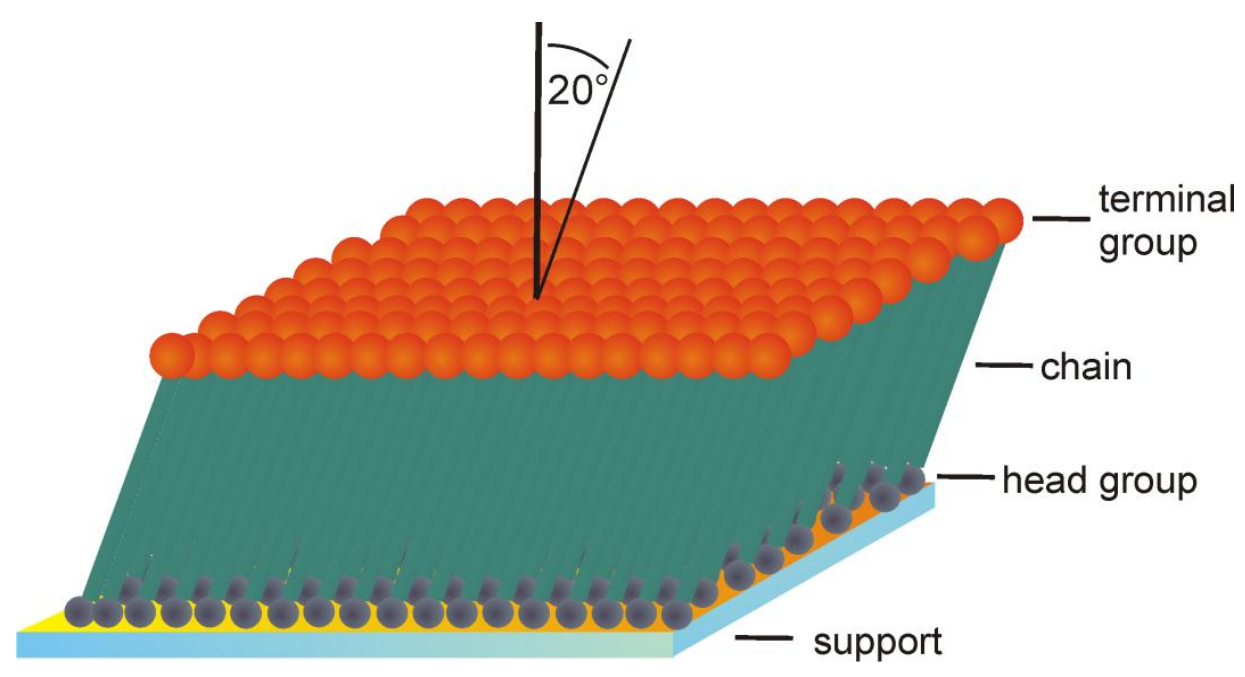

Fig. 3.5 Scheme of a self assembled monolayer (SAM). The head groups (thiol or silane) are chemisorbed on a solid support (gold or silicon/ aluminum oxide) that can be either planar or porous. The chains have a defined length and are tilted $20-30^{\circ}$ from the surface normal. The terminal group is modified in order to create desired surface characteristics.

In this work both thiol and silane chemistry was used to functionalize both planar gold and silicon surfaces and porous aluminum oxide surfaces.

\section{Octanethiol Functionalization of Planar Glass Substrates}

Before surface functionalization of the substrates by means of thiol chemistry could be conducted a defined layer of chromium and gold was evaporated onto the high refractive LaSFN9 glass plates and the AT-cut quartz crystals. The LaSFN9 glass plates that were employed for surface plasmon resonance (SPR) measurements had a square size of $2.5 \times 2.5 \mathrm{~cm}^{2}$, the quartz crystals used for quartz crystal microbalance (QCM) measurements were $1.4 \mathrm{~cm}$ in diameter $(d)$ and had a thickness of $h=0.33 \mathrm{~mm}$. Both substrates were first cleaned in a $5 \%$ Hellmanex $^{\circledR}$ solution via ultrasonication for $15 \mathrm{~min}$ at room temperature. Afterwards they were instantly rinsed with ultrapure deionized water and ultrasonically cleaned in ultrapure, deionized water (MilliQ water) two times for another $15 \mathrm{~min}$. The glass substrates were then separately rinsed with ultrapure, deionized water, dried in a stream of nitrogen and placed inside a Bal-Tec MCS610 thermal evaporation unit equipped with a QSG060 thickness monitor. The 
quartz crystals were mounted inside a master plate followed by evaporation of $10 \mathrm{~nm}$ chromium and $120 \mathrm{~nm}$ gold $\left(5 \cdot 10^{-9}\right.$ bar, $\left.4 \mathrm{~V}\right)$ on each side to obtain a gold area of $A_{\mathrm{g}}$ old $=0.255 \mathrm{~cm}^{2}$ (Fig. 3.6).

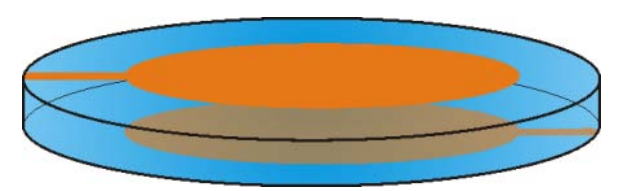

Fig. 3.6 Schematic drawing of an AT-cut quartz crystal $(d=1.4 \mathrm{~cm}, h=0.33 \mathrm{~mm})$ evaporated with a defined layer of chromium and gold on each side $\left(A_{\text {gold }}=0.255 \mathrm{~cm}^{2}\right)$.

In case of the LaSFN9 plates a $1.5 \mathrm{~nm}$ chromium layer and a layer of 45-55 $\mathrm{nm}$ of gold were applied to the plates. To provide a smooth and uniform gold layer the substrates were rotated during the evaporation process. The quality of the gold layer was controlled using a light microscope.

To hydrophobically functionalize the gold surface of both the AT-cut quartz crystals and the LaSFN9 glass plates, they were first treated with Ar plasma and then immersed in a $10 \mathrm{mM}$ ethanolic solution of octanethiol for two hours at room temperature or $12 \mathrm{~h}$ at $4{ }^{\circ} \mathrm{C}$. The substrates were thoroughly rinsed with ethanol p.a. and buffer. Formation of the self assembled monolayer (SAM) occurred as a result of a spontaneous thiol chemisorption onto the gold surface and dense packing of the alkane chains due to van der Waals interaction. ${ }^{16}$ The quality of the SAM was controlled by means of impedance spectroscopy (see chapter 3.4.1). The octanethiol-gold substrates were used immediately after functionalization to prevent any contamination that might affect the process of vesicle spreading (see chapter 3.2.3).

The SAM of the quartz crystal gold surface could be chemically removed using Piranha solution (conc. $\mathrm{H}_{2} \mathrm{SO}_{4} / 30 \% \mathrm{H}_{2} \mathrm{O}_{2}, 3: 1, v / v$ ) for two minutes. After the incubation in Piranha solution the quartz crystals were subsequently rinsed with MilliQ water and dried under a stream of nitrogen. Due to an increased surface roughness after this procedure, 
the gold coated quartz crystals were only reused as long as a sufficient quality could be guaranteed. The quality of the gold layer was again controlled by means of light microscopy.

If the quality of the gold layer was inadequate after treatment with Piranha solution, the gold and the underlying chromium were removed and the substrates recoated with chromium and gold. Therefore, the substrates were incubated in a $0.5 \mathrm{M} \mathrm{KI} / 0.25 \mathrm{M} \mathrm{I} 2$ solution for $20 \mathrm{~min}$ at room temperature to remove the gold layer and a solution composed of $5 \mathrm{M} \mathrm{KOH}$ and $1 \mathrm{M} \mathrm{K}_{3}\left[\mathrm{Fe}(\mathrm{CN})_{6}\right]$ to remove the chromium layer.

The octanethiol monolayer that covered the LaSFN9 glass plates could not be removed via Piranha treatment, because the high refractive index glass is not resistant against strong acid solutions. Instead, the gold and chromium layer was removed after each functionalization and the glasses were recoated as described above.

\section{Dodecyl-trichlorosilane Functionalization of Silicon and Anodic Aluminum Oxide Substrates}

Planar silicon wafers $\left(1.8 \times 0.5 \mathrm{~cm}^{2}\right)$ covered with $100 \mathrm{~nm} \mathrm{SiO} 2$ or porous anodic aluminum oxide (AAO) substrates (see chapter 3.1.1) were functionalized with dodecyltrichlorosilane (DTS) to ensure similar hydrophobicity to the octanethiol covered gold surfaces. Silane chemistry was used instead of thiol chemistry to retain the optical properties of the respective substrates in order to perform fluorescence microscopy and optical waveguide spectroscopy (OWS) experiments. Prior to functionalization, the silicon wafers were rinsed thoroughly with isopropanol and MilliQ water. They were immersed in a solution of $\mathrm{H}_{2} \mathrm{O} / \mathrm{NH}_{3} / \mathrm{H}_{2} \mathrm{O}_{2}(5: 1: 1, v / v / v)$ for 20 min at $70{ }^{\circ} \mathrm{C}$ to obtain a clean and hydrophilic surface. In case of the AAO substrates hydrophilization was achieved via $\mathrm{O}_{2}$ plasma treatment for $3 \mathrm{~min}$. For DTS functionalization both substrates were incubated in dry toluene with 2 vol.\% DTS for 15 min under vacuum, immediately rinsed with dry toluene and left overnight in vacuum at $65{ }^{\circ} \mathrm{C}$ according to the literature. ${ }^{17-19}$ The functionalized substrates were stored under air atmosphere for no longer than two 
days before usage. Toluene was dried over $0.4 \mathrm{~nm}$ molecular sieve (Roth, Karlsruhe, Germany) and kept no longer than two weeks before using.

\section{Aminopropyl-triethoxysilane Functionalization of Anodic Aluminum Oxide Substrates}

To obtain a positively charged, hydrophilic surface at physiological conditions ( $\mathrm{pH} 7.4)$ aminopropyl-triethoxysilane (APTES) was used as silane reagent to functionalize the anodic aluminum oxide $(\mathrm{AAO})$ pore interior. ${ }^{20}$ In order to silanize the AAO porous substrates, they were first cleaned and hydrophilized via $\mathrm{O}_{2}$ plasma treatment. They were then placed inside a glass staining jar together with $50 \mu \mathrm{l}$ of APTES pipetted in a glass test tube that was also placed inside the jar. The chamber was then sealed with Scotch electrical tape (3M, St-Paul (MN), USA) and left in an oven to heat for $10 \mathrm{~min}$ at $130{ }^{\circ} \mathrm{C}$, followed by $12 \mathrm{~h}$ under constant evacuation, a process that has been described as vapor deposition. ${ }^{21}$ The silanized substrates were stored under air atmosphere for no longer than three days before usage.

\section{Mercaptoethanol Functionalization of Porous Silicon Nitride Substrates}

FluXXion porous silicon nitride substrates (fluXXion B.V, Eindhoven, Netherlands) with a pore diameter of $1.2 \mu \mathrm{m}$ were used for membrane and F-actin/membrane indentation experiments (see Fig. 3.7). The porous area on these substrates is arranged in a pattern of 14 lines with 10 rectangles with the writing "FLUXX SIEVE 1.20" on one side of the solid support. This pattern was used as a coordinate system that allows for localization of membrane patches during the atomic force indentation experiments. 

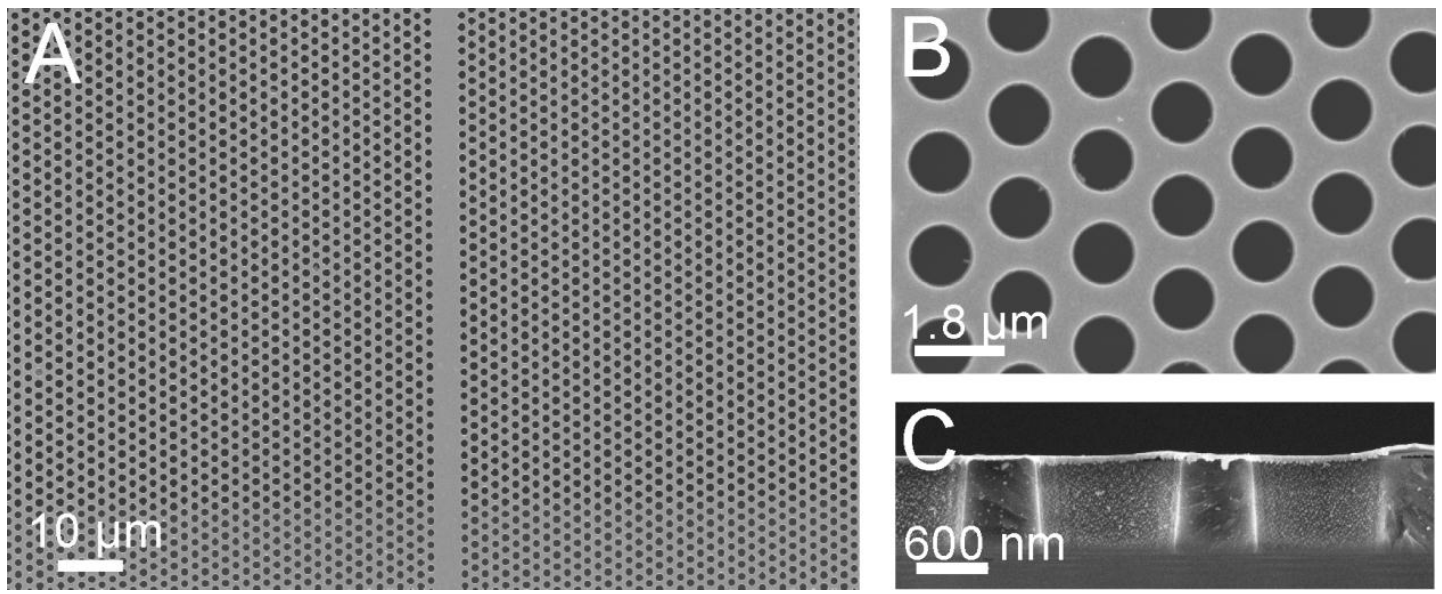

Fig. 3.7 SEM micrograph of porous silicon nitride substrates with a pore diameter of $1.2 \mu \mathrm{m}$. (A) Top view of 2000 times and (B) 25.000 times magnified porous substrates. (C) side view of silicon nitride pores evaporated with a $35 \mathrm{~nm}$ gold layer.

Before functionalization of the fluXXion porous substrates with mercaptoethanol was conducted, they had been coated with a thin adhesive layer of titanium and a 30-35 nm gold layer. First the substrates were treated with Ar plasma for $3 \mathrm{~min}$, then titanium was sputter coated (Sputter Coater 108 Auto, Cressington Instruments, Watfort, UK) on top of nine fluXXion substrates arranged quadratically at $40 \mathrm{~mA}$ for $20 \mathrm{sec}$. The porous substrates were then placed inside the Bal-Tec MCS610 thermal evaporation unit directly beyond the gold evaporation vessel. Without rotation, $30-35 \mathrm{~nm}$ of gold were evaporated onto the substrates (tooling factor: 0.71 ). This procedure was chosen to reduce the penetration depth of gold clusters inside the pores. After the AU evaporation, to ensure that no gold lined the pore interior, the interior was coated with $9 \mathrm{~nm}$ of titanium by sputtering it on the backside of the fluXXion substrates.

The fluxxion substrates were then treated with Ar plasma for $3 \mathrm{~min}$. For hydrophilic functionalization they were immersed in a $10 \mathrm{mM}$ isopropanolic mercaptoethanol solution for $1 \mathrm{~h}$ at room temperature. After incubation, the substrates were thoroughly rinsed with isopropanol and dried under a stream of nitrogen. All substrates were used immediately after functionalization (see chapter 3.2.5) 


\subsection{Lipidchemical Methods}

\subsubsection{Preparation of Lipid Films}

Lipid films were prepared from stock solutions $(2-10 \mathrm{mg} / \mathrm{ml})$ of the particular lipid in chloroform ( $\mathrm{PIP}_{2}$ was dissolved in $\mathrm{CHCl}_{3} / \mathrm{MeOH} / \mathrm{H}_{2} \mathrm{O}, 20: 9: 1$ ). All lipids used in this work were purchased from Avanti Polar Lipids (Alabaster, USA), they are listed in Table 3.1. Corresponding to the desired amount and lipid ratio, certain volumes of the stock solutions were pipetted in a test tube containing $200 \mu$ of chloroform (total lipid concentrations 0.2-0.5 mg). If $\mathrm{PIP}_{2}$ containing lipid films were prepared, a $4 \mu \mathrm{l}$ of $\mathrm{MeOH}$ p.a. was pipetted to the chloroformic solution. The test tubes were transferred to a water bath that had been heated to $32{ }^{\circ} \mathrm{C}$ and the solvent was removed in a gentle stream of nitrogen. To completely remove the remaining solvent, the test tubes were placed inside a vacuum oven at $32{ }^{\circ} \mathrm{C}$ for at least $3 \mathrm{~h}$. They were then sealed with Parafilm ${ }^{\oplus}$ and kept at $4{ }^{\circ} \mathrm{C}$ until used, but not longer than three months. 
Table 3.1 Structural formula and properties of the employed lipids.

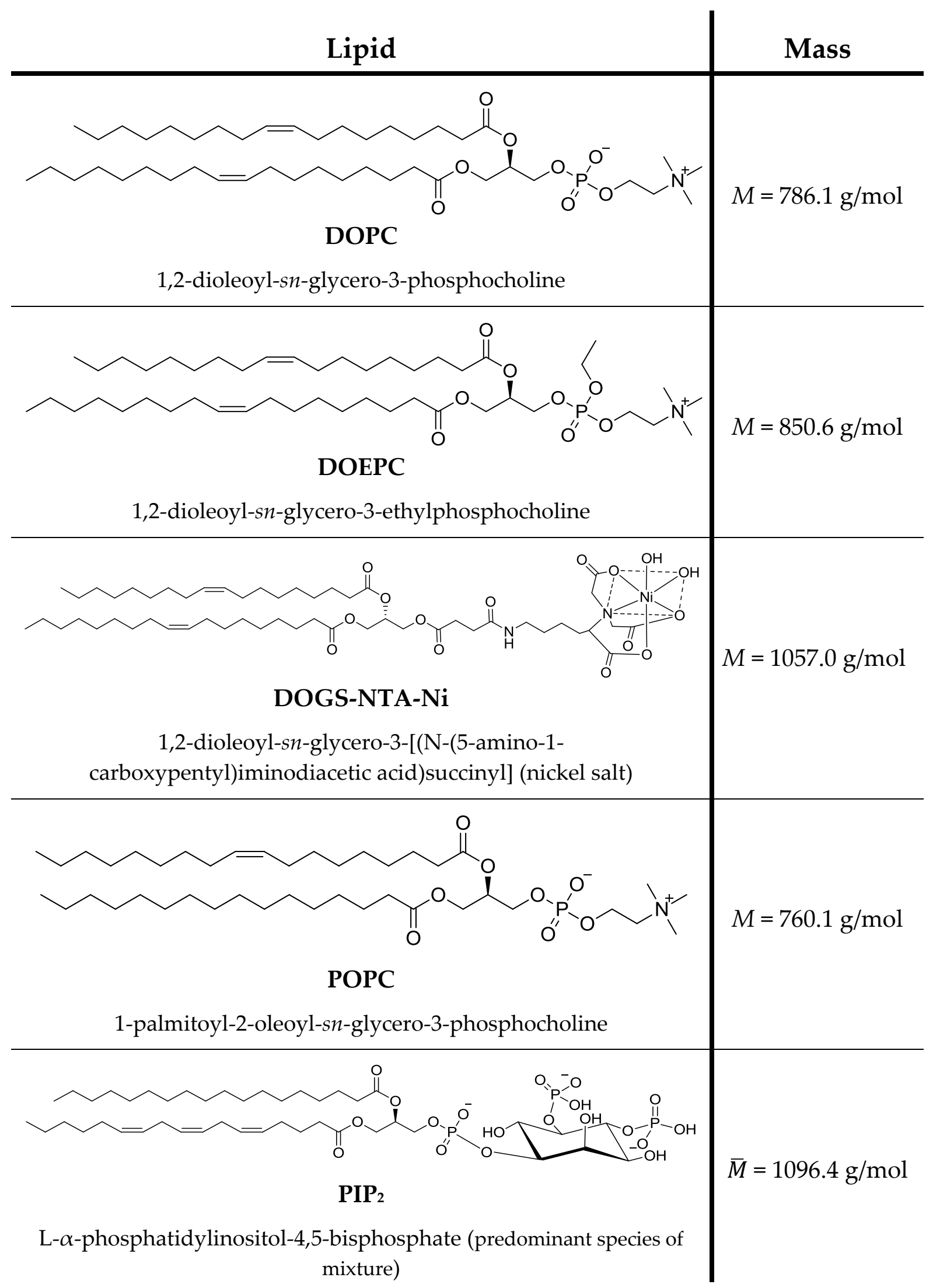


To visualize either the giant unilamellar vesicles (GUVs), solid supported or pore spanning membranes the lipid film was doped with $0.5-1 \%$ of a fluorescently labeled lipid marker (see Table 3.2).

Table 3.2 Structural formula and properties of the employed fluorescent lipid marker.

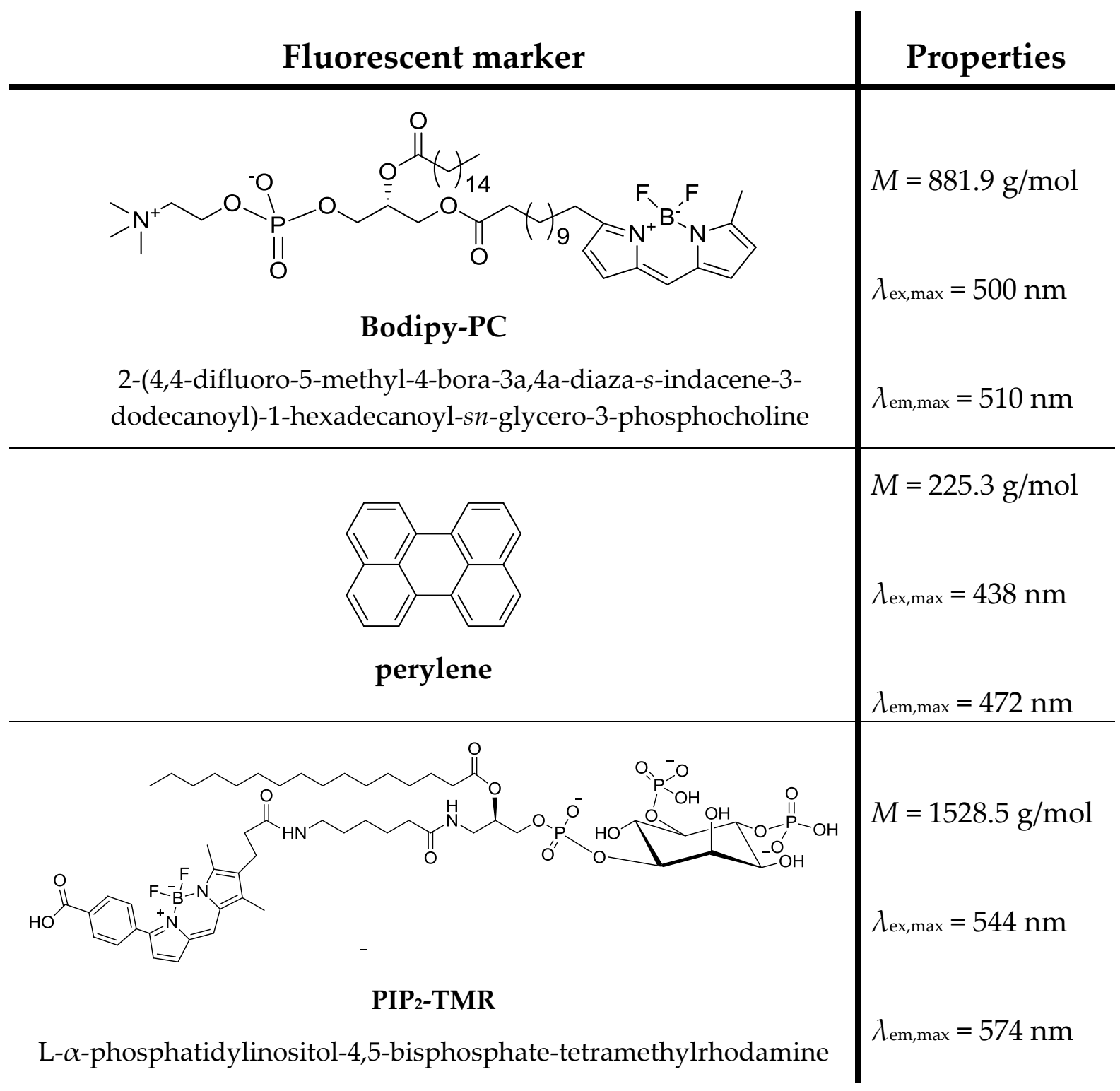




\subsubsection{Preparation of Unilamellar Vesicles}

Unilamellar vesicles have been used as a model membrane system to investigate the properties of biological membranes. Unilamellar vesicles are often used to reconstitute transmembrane proteins and due to their defined, separated inner compartment as a drug delivery system. The can be classified according to their size: small unilamellar vesicles (SUVs) have a diameter ranging from $20-50 \mathrm{~nm}$, large unilamellar vesicles (LUVs) have a diameter of 50-1000 nm and giant unilamellar vesicles (GUVs) are between 1-100 $\mu \mathrm{m}$ in diameter. While SUVs and LUVs are often employed to create solid supported membranes from vesicle spreading, GUVs can directly be addressed with an optical microscope. Also, GUVs are used to create pore spanning membranes via spreading technique.22

\section{Small Unilamellar Vesicles}

Small unilamellar vesicles (SUVs) were prepared via sonication to obtain liposomes in the range of $20-50 \mathrm{~nm}$ in diameter. A lipid film (see chapter 3.2.1) was rehydrated in at least $0.5-1 \mathrm{ml}$ of the desired buffer and left for $20 \mathrm{~min}$. The suspension was then vortexed three times for $30 \mathrm{sec}$ in intervals of $5 \mathrm{~min}$. The so formed multilamellar vesicles (MLVs) were transferred to an Eppendorf ${ }^{\oplus}$ tube and sonicated for $3 \times 10$ min using a tip-sonifier (Sonoplus HD 2070) from Bandelin (Berlin, Germany) with a pulsation cylce of $40 \%$ and amplitude of $65 \%$. Between each sonification step the water inside the sonifier was renewed to prevent any damage of the lipid material caused by water heating during sonication. SUV concentration and buffer composition differed according to the conditions needed for each individual spreading experiment.

\section{Giant Unilamellar Vesicles}

Giant unilamellar vesicles (GUVs) were prepared by the electroformation technique as described by Mathivet et al. in $1996 .{ }^{23}$ At first lipid stock solutions in chloroform and a 
fluorescent marker lipid were mixed with pure chloroform p.a. in the desired ratio (final mass $=0.15 \mathrm{mg}$, total volume $=60 \mu \mathrm{l}) .30 \mu \mathrm{l}$ of this stock solution was pipetted on each of the two cover slides that were covered with a thin, electrical conductive film of indium tin oxide (ITO slide). The solvent was removed under vacuum for $30 \mathrm{~min}$ at room temperature. The ITO glass slides were then assembled as depicted in Fig. 3.8. Electrical contact was ensured by application of a copper stripe. A $300 \mathrm{~mm}$ solution of sucrose was added to rehydrate the lipid film and an AC voltage of $3 \mathrm{~V}$ with a frequency of $5 \mathrm{~Hz}$ was applied for $3 \mathrm{~h}$ to allow for GUV formation.

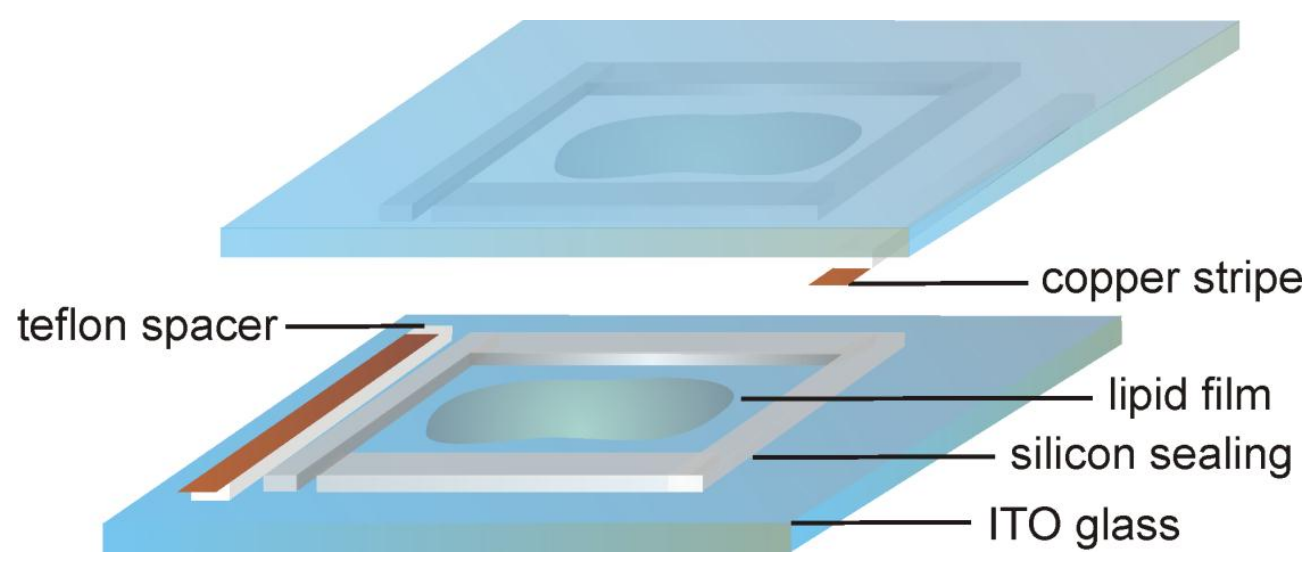

Fig. 3.8 Scheme of ITO slides assembly used as a GUV preparation chamber equipped with teflon spacers and copper stripes to achieve electrical conductivity. The lipid film is deposited between the silicon sealing.

\subsubsection{Solid Supported Monolayers on Functionalized Surfaces}

Solid supported hybrid membranes can be fabricated by spreading small or large unilamellar vesicles on a hydrophobically functionalized surface (see chapter 3.1.2) Due to the hydrophilic headgroups in the outer leaflet, membrane formation cannot take place via simple vesicle adsorption and spreading. A mechanism of liposome fusion onto hydrophobic surfaces was first suggested by Lingler et al. in 1997 as a process that involves vesicle adhesion, rupturing and lateral spreading of the monolayer. ${ }^{24}$ The 
main driving force of this process is presumably a hydrophobic effect, as the addition of a lipid adlayer on a hydrophobic alkanethiol surface causes a reduction of free energy at the SAM/water interface..$^{25}$ Some of the advantages of hybrid membranes compared to solid supported bilayers are the easy fabrication, an increased mechanical stability and the possibility of applying electrochemical techniques and biosensor techniques such as SPR or QCM, if gold is used as a solid support. ${ }^{25}$ Hybrid membranes are limited by the rigidity of the solid support, which results in a reduced fluidity and hinders the insertion of membrane proteins. ${ }^{26}$

\section{Planar Octanethiol Functionalized Gold Surfaces}

Gold coated planar AT-cut quartz crystals for quartz crystal microbalance (QCM) experiments and high refractive LaSFN9 glass slides for surface plasmon resonance (SPR) experiments were functionalized with octanethiol as described in chapter 3.1.2.

In case of the AT-cut quartz crystals, a SUV solution $(c=0.3 \mathrm{mg} / \mathrm{ml})$ was added to the hydrophobic SAM at room temperature, immediately after the formation of the self assembled octanethiol monolayer was controlled by means of impedance spectroscopy. After an incubation time of $1.5 \mathrm{~h}$, the system was thoroughly rinsed with buffer and the quality of the formed membrane was checked by means of impedance spectroscopy (see chapter 3.4.1).

In case of the LaSFN9 glass plates a $0.3 \mathrm{mg} / \mathrm{ml}$ SUV solution was pumped via a peristaltic pump to the octanethiol covered gold plate for $1-1.5 \mathrm{~h}$ at room temperature. The spreading process was monitored in situ during the SPR experiments in kinetic mode. After vesicle spreading was finished, buffer was pumped through the system for at least $15 \mathrm{~min}$ to remove vesicles that might be adhered on the membrane surface. Thickness and hence an adequate membrane quality was confirmed by fitting and comparing the SPR spectra taken before and after vesicle spreading (see chapter 3.4.5). SUVs were prepared as described in chapter 3.2.2. 
For experiments regarding the interaction of C-Polycystin-2 with C-Polycystin-1 and PIGEA14, SUVs composed of DOPC/DOGS-NTA-Ni, 9:1 in high salt P1-buffer were used. In case of the experiments performed to study the binding of ezrin and filamentous actin, SUVs composed of POPC/PIP2, 96:4 or DOPC/DOEPC, 7:3 were applied in E1 buffer.

P1 buffer: $\quad 500 \mathrm{mM} \mathrm{NaCl}, 20 \mathrm{mM}$ TRIS/HCl, pH 8.0

E1 buffer: $\quad 50 \mathrm{mM} \mathrm{KCl}, 20 \mathrm{mM}$ TRIS/HCl, $0.1 \mathrm{~mm}$ EDTA, $0.1 \mathrm{~mm} \mathrm{NaN}$, pH 7.4

Planar DTS Functionalized Silicon and AAO Surfaces

Functionalization of planar silicon and porous AAO surfaces with dodecylthrichlorosilane (DTS) was performed described in chapter 3.1.2. For confocal laser scanning microscopy (CLSM) experiments, SUVs fabricated according to the procedure described in chapter 3.2.2 were doped with $0.5-1 \mathrm{~mol} \%$ of a fluorescent lipid marker (see Table 3.2).

Planar DTS functionalized silicon wafers were incubated with a $0.3 \mathrm{mg} / \mathrm{ml} \mathrm{SUV} \mathrm{solu-}$ tion (DOPC/DOGS-NTA-Ni/Bodipy-PC, 89.5:10:0.5) for $1.5 \mathrm{~h}$ at room temperature and then rinsed with high salt P1 buffer. Membrane quality was controlled by means of CLSM images and fluorescence recovery after photobleaching (FRAP) experiments (see chapter 3.4.7).

Porous DTS functionalized AAO substrates used for CLSM experiments were incubated with $0.4 \mathrm{mg} / \mathrm{ml} \mathrm{SUV} \mathrm{solution} \mathrm{for} \mathrm{at} \mathrm{least} 2.5 \mathrm{hr}$ at room temperature. The larger SUV concentration and longer incubation time was necessary to guarantee the formation of a continuous membrane that lines the whole pore interior. The substrates were then rinsed with buffer and the quality of the membrane was controlled by means of CLSM. 
For porous DTS functionalized AAO substrates used for optical waveguide spectroscopy (OWS) experiments SUV spreading was followed in situ. For that purpose the OWS kinetic mode was used with a setup that corresponds to the SPR experimental design. The $0.4 \mathrm{mg} / \mathrm{ml}$ solution was pumped through the system for at least $1.5 \mathrm{~h}$ until vesicle spreading was finished. Buffer rinsing ensured the removal of unspecifically adsorbed vesicles. OWS spectra that were taken before and after vesicle spreading served as indication for an appropriate membrane quality.

SUVs composed of POPC/PIP2 (9:1) or DOPC/DOEPC (7:3) in E1 buffer were used for experiments performed with porous DTS-AAO substrates. For CLSM experiments, the SUVs were doped with either $0.5 \mathrm{~mol} \%$ Bodipy-PC or $1 \mathrm{~mol} \%$ perylene.

\subsubsection{Solid Supported Bilayers on Silicon Substrates}

Solid supported lipid bilayers can be formed by vesicle spreading on clean, hydrophilic surfaces such as silica/oxidized silicon, mica and titanium oxide. ${ }^{26,27}$ On the contrary to hybrid solid supported membranes the fluidity of the membrane is maintained by a 1-2 $\mathrm{nm}$ water layer trapped underneath the membrane $\left(D=1-4 \mu^{2} / \mathrm{sec}\right)$. Also, the membrane surface is more smooth than a gold-alkenathiol/monolayer surface, which allows for atomic force microscopy imaging.

Solid supported lipid bilayers were obtained by spreading SUVs with a $0 \mathrm{~mol} \%$, $10 \mathrm{~mol} \%, 20 \mathrm{~mol} \%$ and $30 \mathrm{~mol} \%$ DOEPC content (matrix lipid: DOPC) that were doped with $1 \mathrm{~mol} \%$ perylene or $0.5 \mathrm{~mol} \%$ Bodipy-PC on hydrophilic silicon wafers covered with $100 \mathrm{~nm} \mathrm{SiO}_{2}$.

First, silicon wafers that were cleaned with MilliQ water and isopropanole, were immersed in a solution of $\mathrm{H}_{2} \mathrm{O} / \mathrm{NH}_{3} / \mathrm{H}_{2} \mathrm{O}_{2}(5: 1: 1, v / v / v)$ for $20 \mathrm{~min}$ at $70{ }^{\circ} \mathrm{C}$. To the hydrophilic wafer a SUV solution $(c=0.15 \mathrm{mg} / \mathrm{ml})$ was added in E1 buffer and incubated at room temperature for $1 \mathrm{~h}$. Remaining vesicles were rinsed away with E1 buffer. The 
membrane quality was checked by means of fluorescence microscopy and FRAP experiments.

\subsubsection{Pore Spanning Membranes on Silicon Nitride Substrates}

Membranes prepared on a porous support (either porous anodic aluminum oxide or porous silicon substrates) exhibit versatile benefits compared to solid supported membranes. ${ }^{28}$ On the one hand pore spanning membranes (PSMs) are accessible from both the upper and the underlying compartment and can therefore be addressed electrically. Also a solvent free preparation allows for membrane protein incorporation. On the other hand, the solid support facilitates the formation of membranes that obtain long term stability

PSMs were obtained by spreading giant unilamellar vesicles (GUVs, see chapter 3.2.2) on porous silicon nitride substrates (fluXXion substrates, $d_{\text {Pore }}=1.2 \mu \mathrm{m}$ ) that were hydrophilized with mercaptoethanol (see chapter 3.1.2). In contrast to PSMs formed on hydrophobically functionalized fluXXion substrates exhibit a negligible prestres. The hydrophilic porous surface leads to the formation of rather flat PSMs as shown in Fig. 3.9. ${ }^{29,22}$ A freshly functionalized fluxXXon substrate was placed inside a teflon chamber filled with PBS buffer. Before fixing the substrate, it was precautiously hit against the teflon bottom to allow the release of air bubbles trapped inside the pores. After fixing the fluXXion substrate, $30 \mu \mathrm{l}$ of the GUV solution that was kept at $4{ }^{\circ} \mathrm{C}$ for no longer than a week was pipetted on the porous substrate. After $1 \mathrm{~h}$ at room temperature the surface of the fluXXion pores was thoroughly rinsed with buffer and the quality of the PSMs was inspected by means of fluorescence microscopy. 


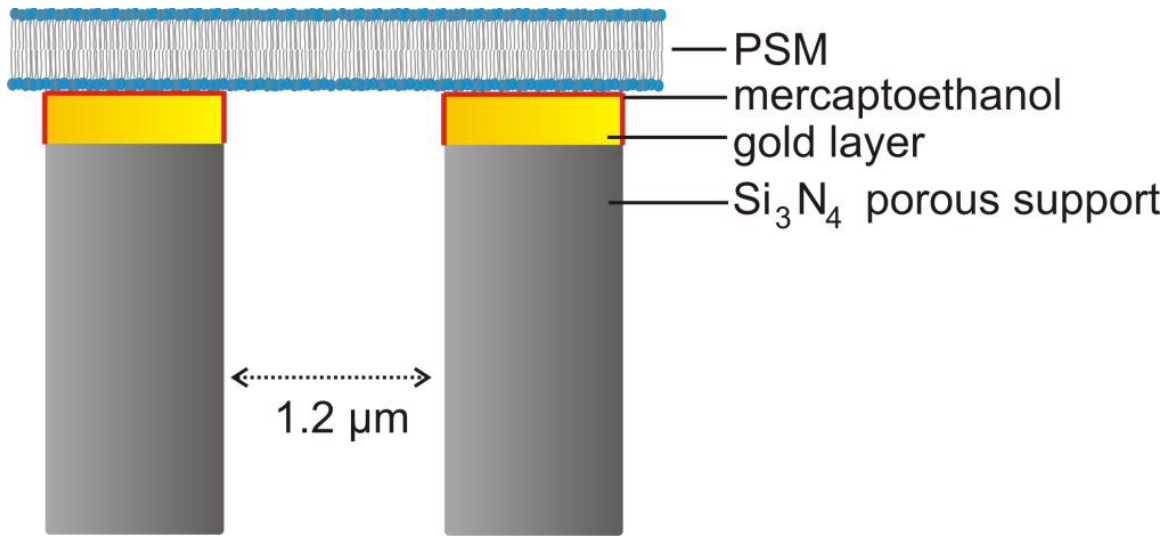

Fig. 3.9 Scheme of a pore spanning membrane. Fluxxion porous substrates with a pore diameter of $1.2 \mu \mathrm{m}$, coated with a $30 \mathrm{~nm}$ layer of gold and functionalized with mercaptoethanol were used.

PBS buffer:

$2.7 \mathrm{mM} \quad \mathrm{KCl}, \quad 136.9 \mathrm{mM} \quad \mathrm{NaCl}, \quad 1.5 \mathrm{mM} \quad \mathrm{KH}_{2} \mathrm{PO}_{4}, 8.1 \mathrm{~mm}$ $\mathrm{Na}_{2} \mathrm{HPO}_{4}, \mathrm{pH} 7,4$

\subsection{Proteinbiochemical Methods}

There are several well known protein expression systems available. Alongside the most prominent prokaryotic system Escherchia coli (E. coli), eukaryotic model organisms like yeast (i.e. Saccharomyces cerevisiae, Pichia pastoris), insect cells or animal cells (Chinese hamster ovary-, HeLa-cells) are employed.

Recombinant protein expression in E. coli allows rapid protein synthesis up to high concentrations (40-50 \% of the total cell protein) with a large number of available cloning vectors. ${ }^{30}$ The E. coli strain BL21 is convenient for protein expression using the common T7 promoter. In the recent past, the pET vector (from Novagen, Madison, USA) has become more and more popular (Fig. 3.10). In this vector system the basal transcription via T7 polymerase (DE3 fragment encodes for T7 polymerase and LacI 
repressor) without induction is inhibited via the T7 lysozyme. Lysozyme expression takes place via an additional plasmid called pLys..$^{31,32}$

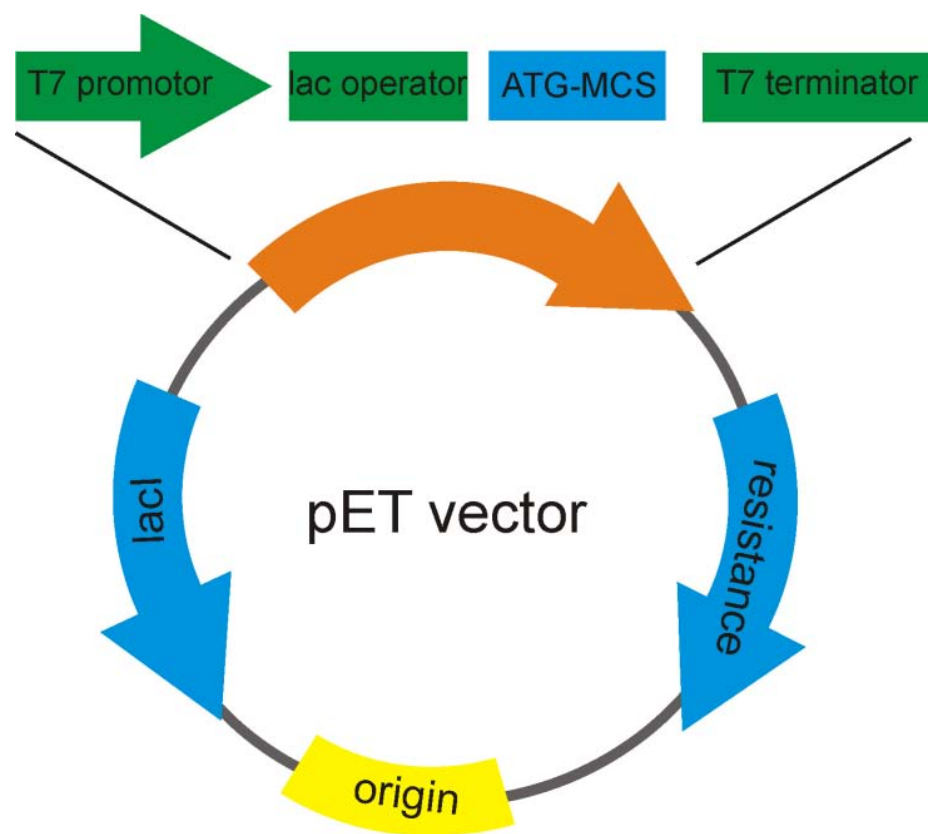

Fig. 3.10 pET vector with the origin of transcription (yellow), genes for lacI repressor and resistance marker (blue) and the gene of interest (orange) containing a T7 promoter, lac operator, the multiple cloning site for the target gene and the T7 terminator site.

Drawbacks of the E. coli expression system are the formation of inclusion bo-dies ${ }^{31}$ and the lack of posttranslational modifications. To overcome these disadvantages, eukaryotic model organisms can be utilized.

The proteins that were recombinantly expressed in E. coli in this work, are listed together with their properties, strains, vectors and antibiotic resistances in Table 3.3. 
Table 3.3 Proteins that were recombinantly expressed in E. coli, respective strains, vectors and resistances. Protein properties were extracted from expasy official website. ${ }^{33}(\mathrm{amp} .=$ ampicillin, cam. $=$ chloramphenicol, kan. $=$ kanamycin $)$

\begin{tabular}{|c|c|c|}
\hline Protein & Properties & Strain, vector,resistance \\
\hline $\begin{array}{c}\text { cPC2 } \\
\text { C-terminal polycystin-2 }\end{array}$ & $\begin{array}{c}290 \text { amino acids } \\
M=32.69 \mathrm{kDa} \\
\text { pI } 5.06 \\
\varepsilon=11,460 \mathrm{M}^{-1} \cdot \mathrm{cm}^{-1}\end{array}$ & $\begin{array}{c}\text { Rosetta, } \\
\text { pMAl-c2/TEV-PKD2c } \\
\text { amp./cam. }\end{array}$ \\
\hline $\begin{array}{c}\text { cPC1 } \\
\text { C-terminal polycystin-1 }\end{array}$ & $\begin{array}{c}134 \text { amino acids } \\
M=14.58 \mathrm{kDa} \\
\mathrm{pI} 10.98 \\
\varepsilon=1,490 \mathrm{M}^{-1} \cdot \mathrm{cm}^{-1}\end{array}$ & $\begin{array}{c}\text { BL21(DE3), } \\
\text { pET21b/PKD1c } \\
\text { amp. }\end{array}$ \\
\hline $\begin{array}{l}\text { PIGEA14 } \\
\text { polycystin-2 interactor, } \\
\text { Golgi- and endoplasmic } \\
\text { reticulum-associated pro- } \\
\text { tein with a mass of } 14 \mathrm{kDa}\end{array}$ & $\begin{array}{c}126 \text { amino acids } \\
\begin{array}{c}M= \\
\text { pI } 9.09 \\
\varepsilon=6,990 \mathrm{M}^{-1} \cdot \mathrm{cm}^{-1}\end{array}\end{array}$ & $\begin{array}{c}\text { BL21(DE3), } \\
\text { pET21b/CIP1(116/493) } \\
\text { amp. }\end{array}$ \\
\hline $\begin{array}{l}\text { ezrin wt, } \\
\text { ezrin T567A, } \\
\text { ezrin T567D }\end{array}$ & $\begin{array}{c}586 \text { amino acids } \\
\begin{array}{c}M=69.41 \mathrm{kDa} \\
\text { pI } 5.94 \\
\varepsilon=66,935 \mathrm{M}^{-1} \cdot \mathrm{cm}^{-1}\end{array}\end{array}$ & $\begin{array}{c}\text { BL21(DE3)pLysS, } \\
\text { pET28a (+) } \\
\text { kan./cam. }\end{array}$ \\
\hline
\end{tabular}




\subsubsection{Heterologous Protein Expression in Escherichia coli}

E. coli bacterial strains were kept as glycerol stocks at $-80{ }^{\circ} \mathrm{C}$ prepared from overnight cultures and sterile glycerol, 1:1 (v/v). From these stocks, bacteria colonies were transferred to a lysogeny broth (LB) containing agar plate using the streaking technique. The agar plate was incubated for $12-15 \mathrm{~h}$ at $37^{\circ} \mathrm{C}$. For overnight cultures $10 \mathrm{ml}$ of LB medium was inoculated with an individual colony and incubated overnight at $37^{\circ} \mathrm{C}$ and $150 \mathrm{rpm}$. The main culture $(250 \mathrm{ml})$ was inoculated with $5 \mathrm{ml}$ of the overnight culture (in case of cPC2-MBP fusion protein $0.5 \mathrm{~g} / 1$ glucose was added to the main culture) and incubated at $37^{\circ} \mathrm{C}$ and $150 \mathrm{rpm}$ until an optical density at $\lambda=595 \mathrm{~nm}$ (OD $\left.{ }_{595}\right)$ of 0.5-0.6 was reached (in case of $\mathrm{cPC1}$ : $\mathrm{OD}_{595}=1.0$ ). Protein expression was induced by addition of $1 \mathrm{mM}$ isopropyl $\beta$-D-1-thiogalactopyranoside (IPTG) and the main cultures were incubated at $37^{\circ} \mathrm{C}$ and $150 \mathrm{rpm}$ for $4 \mathrm{~h}$ (in case of PIGEA14: $T=30^{\circ} \mathrm{C}$, in case of ezrin: cells were harvested after $3 \mathrm{~h}$ ). The respective antibiotics (as listed in Table 3.3) were given to the LB media of agar plate, overnight and main culture before further usage with the following final concentrations: ampillicin (amp.), $\mathrm{c}=50 \mu \mathrm{g} / \mathrm{ml}$; kanamycin (kan.), c = $60 \mu \mathrm{g} / \mathrm{ml}$; chloramphenicol (cam.), c = $34 \mu \mathrm{g} / \mathrm{ml}$. After incubation with IPTG, the main culture was allowed to cool on ice for $15 \mathrm{~min}$. Cells were harvested via centrifugation $\left(4000 \times \mathrm{g}, 4^{\circ} \mathrm{C}, 20 \mathrm{~min}\right)$. The cell pellet was resuspended in lysis buffer and sonicated on ice $(5 \times 30 \mathrm{~s}$, duty cycle: $50 \%$, output control 5$)$. The cell lysates were centrifuged at $100000 \times \mathrm{g}$ and $4{ }^{\circ} \mathrm{C}$ for $1 \mathrm{~h}$.

LB medium: $\quad 10 \mathrm{~g} / \mathrm{l}$ bacto tryptone, $5 \mathrm{~g} / \mathrm{l}$ yeast extract, $10 \mathrm{~g} / \mathrm{l} \mathrm{NaCl}, \mathrm{pH} 7.4$ $(\mathrm{NaOH})$

LB agar: $\quad 15 \mathrm{~g} / \mathrm{l}$ agar, $10 \mathrm{~g} / 1$ bacto tryptone, $5 \mathrm{~g} / 1$ yeast extract, $10 \mathrm{~g} / \mathrm{l}$ $\mathrm{NaCl}, \mathrm{pH} 7.4(\mathrm{NaOH})$ 
lysis buffer $\quad 500 \mathrm{mM} \mathrm{NaCl}, 5 \mathrm{mM}$ imidazole, $20 \mathrm{~mm}$ TRIS/HCl, pH 8.0 (cPC1,PIGEA14):

lysis buffer $\quad 200 \mathrm{mM} \mathrm{NaCl}, 20 \mathrm{mM}$ TRIS/HCl, $1 \mathrm{mM}$ EDTA, pH 7.4 (cPC2):

lysis buffer (ez- $300 \mathrm{mM} \mathrm{NaCl}, 20 \mathrm{~mm}$ imidazole, $40 \mathrm{~mm}$ HEPES, 1 mM EDTA, rin): $10 \mathrm{mM}$ mercaptoethanol (right before usage), 1 tablet Roche complete protease inhibitor $/ 50 \mathrm{ml}$ (right before usage)

stock solutions: $50 \mathrm{mg} / \mathrm{ml}$ amp., $30 \mathrm{mg} / \mathrm{ml}$ kan., $34 \mathrm{mg} / \mathrm{ml}$ cam. (in ethanol p.a.), 1 M IPTG

\subsubsection{Protein Purification and TEV Protease Cleavage}

All proteins listed in Table 3.3 had a C-terminal (cPC1 and PIGEA14) or N-terminal (ezrin) hexahistidine (His6) tag, except cPC2 that was recombinantly expressed as a maltose binding protein (MBP) fusion protein to increase protein stability and facilitate correct protein folding. In case of the His6 tagged proteins, the supernatant obtained after ultracentrifugation was applied to a nitrilotriacetic acid (Ni-NTA) agarose column (Qiagen, Hilden, Germany) or an iminodiacetic acid (Ni-IDA) agarose column (Novagen, Darmstadt, Germany) that had first been charged with $50 \mathrm{~mm} \mathrm{NiSO} 4$ (column volume $=1.5-2 \mathrm{ml})$. All columns were washed with 10 column volumes of MilliQ water and equilibrated with 15 column volumes of equilibration buffer before applying the supernatant (in case of the ezrin purification the column was washed with 10 column volumes of binding buffer after the equilibration step). After the supernatant was applied to the column material, it was washed with 15 column volumes of washing buffer and then eluted with elution buffer. At least 15 fractions (volume per fraction = column volume) were collected.

As cPC2 was expressed with an MBP tag, the fusion protein was first purified using an amylose column from New England Biolabs (Frankfurt, Germany,) with a column vol- 
ume of $1.5 \mathrm{ml}$. The column was prepared by washing with MiIlliQ water and equilibration buffer. After supernatant application, the column was washed with 15 column volumes of washing buffer and the fusion protein was eluted using the respective maltose containing elution buffer. The $\mathrm{CPC} 2$ protein was then cleaved from the MBP using the Tobacco etch virus (TEV) protease $(1: 100, w / w)$. The protein/protease mixture was stirred for $24 \mathrm{~h}$ at room temperature in the presence of dithiothreitol $(1: 50, v / v)$ and EDTA $(1: 50, v / v)$. The cleaved protein was then dialysed against storage buffer and purified using a Ni-IDA agarose column that had been first charged using $50 \mathrm{mM} \mathrm{NiSO}_{4}$ and was then equilibrated with 15 bed volumes of lysis buffer. After the protein lysate was applied to the column, it was washed first with 15 column volumes of storage buffer, then eluted with storage buffer containing $30 \mathrm{~mm}$ imidazole.

All protein purification steps were carried out at $4{ }^{\circ} \mathrm{C}$. Cell lysate, washing and elution fractions were analyzed by SDS-PAGE (chapter 3.3.3).

buffers for ezrin purification:

equillibration buffer: $300 \mathrm{mM} \mathrm{NaCl}, 20 \mathrm{mM}$ imidazole, $40 \mathrm{mM}$ HEPES, $0.1 \mathrm{~mm}$ EDTA, pH 7.4

binding buffer: $\quad 300 \mathrm{mM} \mathrm{NaCl}, 20 \mathrm{mM}$ imidazole, $40 \mathrm{~mm}$ HEPES, $0.1 \mathrm{~mm}$ EDTA, $10 \mathrm{~mm}$ mercaptoethanol (right before usage), $\mathrm{pH} 7.4$

washing buffer: $\quad 300 \mathrm{mM} \mathrm{NaCl}, 30 \mathrm{mM}$ imidazole, $40 \mathrm{mM}$ HEPES, $1 \mathrm{~mm}$ $\mathrm{MgCl}_{2}, 10 \mathrm{mM}$ mercaptoethanol (right before usage), $\mathrm{pH} 7.4$

elution buffer: $\quad 250 \mathrm{mM}$ imidazole, $50 \mathrm{mM} \mathrm{KCl,} 2 \mathrm{mM}$ TRIS/HCl, pH 7.4 
buffers for $\mathrm{CPC} 1$ and PIGEA14 purification:

equilibration buffer: $\quad 500 \mathrm{mM} \mathrm{NaCl}, 5 \mathrm{~mm}$ imidazole, $20 \mathrm{mM}$ TRIS/HCl, $\mathrm{pH} 8.0$

washing buffer: $\quad 500 \mathrm{mM} \mathrm{NaCl}, 5 \mathrm{~mm}$ imidazole, $20 \mathrm{mM}$ TRIS/HCl, $\mathrm{pH} 8.0$

elution buffer (cPC1): $500 \mathrm{~mm} \mathrm{NaCl}, 1 \mathrm{M}$ imidazole, 20 mM TRIS/HCl, pH 8.0

elution buffer (PI- $500 \mathrm{~mm} \mathrm{NaCl}, 50$ mM EDTA, 20 mM TRIS/HCl, pH 8.0

GEA14):

buffers for $\mathrm{CPC} 2$ purification:

equilibration and $\quad 200 \mathrm{~mm} \mathrm{NaCl}, 1 \mathrm{~mm}$ EDTA, $20 \mathrm{mM}$ TRIS/HCl, pH 7.4

washing buffer

(amylose):

elution buffer (amy- $200 \mathrm{~mm} \mathrm{NaCl}, 1 \mathrm{mM}$ EDTA, $10 \mathrm{~mm}$ maltose, $20 \mathrm{mM}$ lose): TRIS/HCl, $\mathrm{pH} 7.4$

storage buffer $500 \mathrm{mM} \mathrm{NaCl}, 20 \mathrm{~mm}$ TRIS/HCl, pH 8.0

(P1 buffer):

elution buffer

$500 \mathrm{~mm} \mathrm{NaCl}, 30 \mathrm{~mm}$ imidazole, $20 \mathrm{~mm}$ TRIS/HCl, pH 8.0

(Ni-IDA):

\subsubsection{SDS-polyacrylamide Gel Electrophoresis}

The principle of sodium dodecylsulfate polyacrylamide gel electrophoresis (SDSPAGE) is based on separating proteins according to their size after denaturation with SDS through a sieving medium in an electric field. In more detail, SDS as a charged, amphiphatic surfactant molecule binds to the protein via hydrophobic interactions and thus denatures the protein. The intrinsic protein charges are masked by SDS molecules and the protein is denatured. Thus, the rate of protein migration in a polyacrylamide 
gel during electrophoresis is inversely proportional to the protein size/mass. Polyacrylamide is formed by polymerization of acrylamide and the crosslinker $N, N^{\prime}$ methylenebisacrylamide (bisacrylamide). Polymerization is initiated by ammonium peroxosulfate (APS) and catalyzed by $N, N, N^{\prime}, N^{\prime}$-tetramethylethylenediamine (TEMED).

Usually, the polyacrylamide gel is divided in an upper stacking gel (large pore size, $5 \%$ acrylamide, $\mathrm{pH} 6.8$ ) and a resolving gel with a lower pore size (6-20\% acrylamide, pH 8.8), with chloride ions as the only mobile anion in both gels. The tank buffer contains glycine molecules, which enter the stacking gel during electrophoresis. Due to the relatively low $\mathrm{pH}$, the glycine molecules are zwitterionic inside the stacking gel and therefore migrate relatively slowly (trailing ions), while the chloride ions migrate relatively fast (leading ions). The proteins that are carried along become stacked in a thin distinct layer. When the protein layer reaches the resolving gel, glycine molecules are deprotonated due to the high $\mathrm{pH}$. Both glycine and chloride ions run faster than the proteins and dissipate in the gel. This allows the proteins to unstuck and separate through the small pores of the resolving gel.

In this work, a $17 \%$ polyacrylamide resolving gel was used to confirm the success of cPC1 and PIGEA14 purification, while for the proteins of larger size (ezrin and cPC2) a $12.5 \%$ gel was used. First, the resolving gel was pipetted into the gel chamber and covered with isopropanol to polymerize for $1 \mathrm{~h}$ at room temperature. Then, the stacking gel was pipetted above the resolving gel and a Teflon comb was placed inside the gel to form small wells for the protein samples. After 45 min of stacking gel polymerization, the gel chamber was placed inside a plastic container. Container and the space inside the gel chamber was filled with tank buffer. The comb was pulled out carefully and the prepared protein samples were loaded inside the wells (loading volume was 10-30 $\mu$ l according to the expected protein concentration). One lane was loaded with a molecular weight standard from GE Healthcare to allow the accurate determination of protein mass. To prepare the protein samples, they were mixed with $2 \times$ sample buffer $(1: 1$, $v / v$ ) and heated inside a thermo mixer from Eppendorf (Hamburg, Germany) to $85{ }^{\circ} \mathrm{C}$ 
for $5 \mathrm{~min}$ with $300 \mathrm{rpm}$. Power leads were attached to the gel chamber assembly and a current of $15 \mathrm{~mA}$ per gel was applied. After approximately $1.5 \mathrm{~h}$ the gels were disassembled, washed with ultrapure, deionized water and put inside a plastic container filled with Coomassie Blue staining solution. The container was shaken for $5 \mathrm{~min}$, before the staining solution was replaced against a destaining solution. Gels were incubated in this solution overnight while shaking. They were then stored in ultrapure, deionized water until they were photographed and thus digitized.

$2 \%$ sample buffer $5 \%(w / v)$ SDS, $0.3 \mathrm{M} \mathrm{DTT}, 15 \%(w / v)$ glycerol, $0.02 \%$ $(w / v)$ bromphenol blue, $0.175 \mathrm{M}$ TRIS/HCl, $\mathrm{pH} 6.8$

$5 \%$ stacking gel

$1.4 \mathrm{ml} \mathrm{H} 2 \mathrm{O}, 330 \mu \mathrm{l}$ acrylamide/bisacrylamide (29:1, $30 \%)$, $20 \mu \mathrm{l} 10 \%(w / v)$ SDS, $20 \mu \mathrm{l} 10 \%(w / v)$ APS, $2 \mu \mathrm{l}$ TEMED, $0.25 \mathrm{ml} 1 \mathrm{M}$ TRIS/HCl, pH 6.8

$12.5 \%$ resolving gel $1.6 \mathrm{ml} \mathrm{H} \mathrm{H}_{2} \mathrm{O} 2.1 \mathrm{ml}$ acrylamide/bisacrylamide (29:1, $30 \%$ ), $40 \mu \mathrm{l} 10 \%(w / v)$ SDS, $40 \mu \mathrm{l} 10 \%(w / v)$ APS, $2 \mu \mathrm{l}$ TEMED, $1.3 \mathrm{ml} 1.5 \mathrm{M}$ TRIS/HCl, $\mathrm{pH} 8.8$

$17 \%$ resolving gel $\quad 0.81 \mathrm{ml} \mathrm{H}_{2} \mathrm{O}, 2.89 \mathrm{ml}$ acrylamide/bisacrylamide (29:1, $30 \%), 40 \mu \mathrm{l} 10 \%(w / v)$ SDS, $40 \mu \mathrm{l} 10 \%(w / v)$ APS, $2 \mu \mathrm{l}$ TEMED, $1.3 \mathrm{ml} 1.5 \mathrm{M}$ TRIS/HCl, pH 8.8

tank buffer 25 mM TRIS, 192 mM glycine, $0.1 \%(w / v)$ SDS, pH 8.3

Coomassie Blue $0.5 \%(w / v)$ Coomassie Brilliant Blue R-250, $45 \%$, (v/v ) staining solution methanol, $18.5 \%(v / v)$ glacial acetic acid destaining solution $5 \%(v / v)$ methanol, $7.5 \%(v / v)$ glacial acetic acid 


\subsubsection{Rebuffering Protein Solutions}

In this work, proteins were rebuffered using either dialysis for larger proteins volumes $(>0.5 \mathrm{ml})$ or centrifugal ultrafiltration devices for smaller volumes.

\section{Dialysis}

For rebuffering large protein volumes, dialysis tubings from Carl Roth (Karlsruhe, Germany) with a molecular weight cut of (MWCO) of either 5 or 14 kDa were used depending on the molecular weight of the protein that had to be rebuffered. The tubings were first cut into pieces of 5-10 cm length. They were then soaked in MilliQ water, immersed for $30 \mathrm{~min}$ in a $10 \mathrm{mM} \mathrm{NaHCO}$ solution at $80^{\circ} \mathrm{C}$, two times for $30 \mathrm{~min}$ in a $80{ }^{\circ} \mathrm{C} 10 \mathrm{mM}$ EGTA solution and once for $30 \mathrm{~min}$ at $80^{\circ} \mathrm{C}$ in MilliQ water. The cleaned tubings were then kept in a $10 \mathrm{mM} \mathrm{NaN}_{3}$ solution at $4{ }^{\circ} \mathrm{C}$ until needed. The tubings were filled with protein solution, sealed with plastic clips and transferred to a beaker containing dialysis buffer with 200-500 times the volume of the protein solution. The tubing assembly was stirred for at least $6 \mathrm{~h}$ with three changes of dialysis buffer at $4{ }^{\circ} \mathrm{C}$. Mostly one dialysis step was performed overnight. Rebuffered protein solutions were then filled in a Falcon tube and kept at $4{ }^{\circ} \mathrm{C}$ until usage. Dialysis buffer was either a protein storage buffer (high salt P1-buffer for cPC1, cPC2 and PIGEA and E1 buffer for ezrin) or a buffer needed for certain experimental conditions. Concentration of rebuffered protein samples were determined via UV/Vis spectroscopy or Bradford assay (chapter 3.3.5)

\section{Centrifugal Ultrafiltration}

Rebuffering of small protein volumes were performed using centrifugal ultrafiltration devices from Sartorius (Göttingen, Germany) with a molecular weight cut of (MWCO) of $5 \mathrm{kDa}$. In order to rebuffer protein solution $50-200 \mu \mathrm{l}$ of protein solution was pipetted together with $200 \mu \mathrm{l}$ of the desired buffer inside the ultrafiltration device. The de- 
vice was then centrifuged three times for 10-20 min with an addition of $200 \mu$ desired buffer after each centrifugation cycle. Rebuffered protein solutions were transferred in an Eppendorf $f^{\circledast}$ tube and stored at $4{ }^{\circ} \mathrm{C}$ until needed. The concentration of rebuffered protein samples was determined via UV/Vis spectroscopy or Bradford assay (chapter 3.3.5).

\subsubsection{Quantification of Protein Concentration}

There are many known assays to determine protein concentration, the Lowry assay, Bradford assay, BCA assay and UV-absorbance being the most popular ones. Each assay exhibits individual benefits and limitations. While a UV-absorbance at $280 \mathrm{~nm}$ is performed quickly and accurate if the protein has a known extinction coefficient $(\varepsilon)$, it is impossible, if either $\varepsilon$ or the protein concentration is too low or components that absorb at $280 \mathrm{~nm}$ including imidazole are in the protein buffer ( $A_{280}$ of $250 \mathrm{~mm}$ imidazole $=0.2-0.4) .{ }^{34}$ In these cases an assay developed by Bradford in 1976 would be the method of choice. However, some detergents or surfactants are not compatible with the Bradford assay. In the presence of detergens the Lowry and BCA assay can be applied. Since both assays base on a reaction with a copper containing Buiret chromophore, they are both not compatible with common reduction agents in protein solution such as mercaptoethanol and DTT. Besides, there are other components such as EDTA, TRIS and potassium ions that interfere with the Lowry reagent. Flow charts for selecting the appropriate assay in order to fulfill requirements by sample and buffer composition are provided by Current Protocols in Protein Science. ${ }^{35}$

\section{UV Absorption}

Protein samples of ezrin or CPC2 exhibited an extinction coefficient $(\varepsilon)$ and concentration to be readily measured by UV/Vis spectroscopy. Hence, their concentration was determined by means of UV/Vis spectroscopy/absorption at $280 \mathrm{~nm}$ if there was no imidazole in the protein sample (after rebuffering the purified proteins, see chapter 3.3.4). 
Absorption at $\lambda=280 \mathrm{~nm}$ occurs due to the presence of aromatic amino acids like phenylalanine, tyrosine and tryptophan. UV spectra were usually collected in a quartz cuvette from $350-210 \mathrm{~nm}$. A baseline corresponding to a UV spectrum of pure buffer solution was subtracted from the measured protein spectrum. Protein concentration was calculated using the Beer-Lamberts law (eq. 3.4):

$$
A=-\lg \frac{I}{I_{0}}=\varepsilon \cdot c \cdot l
$$

with $A=$ absorbance, $I=$ intensity of transmitted light, $I_{0}=$ intensity of incident light, $\varepsilon=$ extinction coefficient (Table 3.3), $c=$ protein concentration and $l=$ path length $(1 \mathrm{~cm})$.

As nucleic acids have an absorption maximum at $260 \mathrm{~nm}$, the correlation of $A_{260}$ and $A_{280}$ is a good indication, whether the protein sample is contaminated by nucleic acids. The $A_{280} / A_{260}$ value should be higher than 1.6 to exclude nucleic acid impurities inside the sample. Absorption at $300 \mathrm{~nm}$ or higher indicated the existence of aggregates as an effect of light scattering. ${ }^{36}$

\section{Bradford assay}

Concentration of proteins with low concentration or extinctions coefficient $\varepsilon$ (cPC1 and PIGEA14) or proteins samples including imidazole, were determined by means of the Bradford assay. ${ }^{37,38}$ This assay bases on the binding of Coomassie Brilliant Blue G250 dye to arginine, histidine, tyrosine, phenylalanine and tryptophan residues of the protein. Binding of the dye to a protein causes a shift of absorption maximum from $470 \mathrm{~nm}$ to $595 \mathrm{~nm}$. The dye response to arginine residues is eight times larger than to others, which causes adulterant values if the protein is rich in arginine. In this case other proteins than bovine serum albumin (BSA) have to be used as standards. 
In this work, BSA was the standard protein. From a BSA stock solution in MilliQ $\left(c_{\text {stock }}=10 \mathrm{mg} / \mathrm{ml}\right.$ ) mixtures of BSA and buffer (buffer corresponds to the one the protein was stored in) containing $0-0.8 \mathrm{mg} / \mathrm{ml} \mathrm{BSA}$ were prepared for a calibration curve (final volume of $1 \mathrm{ml}$ ). $1 \mathrm{ml}$ of freshly filtered Bradford assay solution (20\% in MilliQ water, $(v / v))$ was given in a plastic cuvette. Plastic cuvettes were used instead of quartz, because the Coomassie Brilliant Blue dye inside the Bradford assay solution strongly binds to quartz glass. $20 \mu \mathrm{l}$ of the BSA mixtures as well as of the protein sample of unknown concentration were pipetted in each cuvette. The calibration curve and protein samples were thoroughly mixed and incubated for $5 \mathrm{~min}$. Then the absorption of each sample was measured at $595 \mathrm{~nm}$ with a Cary $50 \mathrm{UV}$ spectrometer from Varian. With the calibration curve (the sample containing no BSA was a buffer blank to assign the zero point) it was possible to determine the concentration of the protein sample.

\subsubsection{Ezrin Labeling}

In order to visualize ezrin on a $\mathrm{PIP}_{2}$ containing solid supported membrane as evidence of the ezrin-PIP2 binding, ezrin T567A was fluorescently labeled using Alexa Fluor488 C5-Maleimide (Fig. 3.11) purchased from Invitrogen and Texas Red Maleimide ( $\lambda_{\mathrm{ex}}=583 \mathrm{~nm}, \lambda_{\mathrm{em}}=603 \mathrm{~nm}$ ) from Jena Bioscience. The labeling procedure is described in the following exemplarily for the Alexa Fluor488 C5-maleimide. The used Alexa Fluor488 C5-maleimide fluorophor exhibits an extinction coefficient $\varepsilon=71000 \mathrm{~cm}^{-1} \cdot \mathrm{M}^{-1}$ and excitation and emission maxima of $\lambda_{\mathrm{ex}}=493 \mathrm{~nm}$ and $\lambda_{\mathrm{em}}=581 \mathrm{~nm}$. 


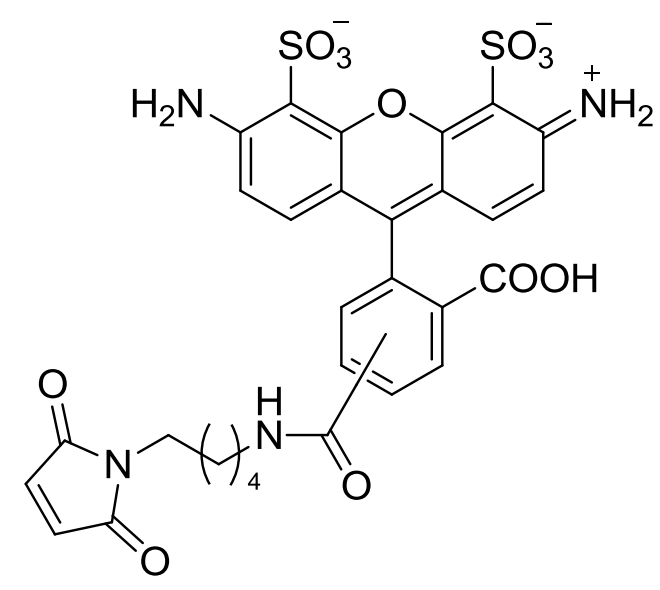

Fig. 3.11 Structural formula of Alexa Fluor488 C5-maleimide.

Protein labeling was conducted according to Bosk et al. and the manual provided by Invitrogen. ${ }^{39,40}$ First, ezrin was concentrated by means of centrifugal ultrafiltration to a final concentration of $19.5 \mu \mathrm{M}$ and a total volume of $1 \mathrm{ml}$ in E1 buffer. A 10-fold molar excess of DTT was added to the solution ( $1.95 \mu \mathrm{l}$ of a $100 \mathrm{mM}$ stock solution) to reduce possible disulfide residues. After $30 \mathrm{~min}$ of incubation, the protein was dialyzed at $4{ }^{\circ} \mathrm{C}$ overnight $(\mathrm{MWCO}=5 \mathrm{kDa}$ ) against DTT free E1 buffer. A 20-fold molar excess of Alexa Fluor488 C5-maleimide ( $28 \mu \mathrm{l}$ of a freshly prepared $10 \mathrm{mg} / \mathrm{ml}$ stock solution in degassed MilliQ water) was added drop wise and stirred overnight at $4{ }^{\circ} \mathrm{C}$. The reaction was quenched by means of $7 \mathrm{mM}$ mercaptoethanol ( $20 \mathrm{~min}$ ). The mixture was then applied to a G-25 Sephadex gel filtration column from GE Healthcare. For that purpose the $1 \mathrm{ml}$ ezrin-AlexaFluo488 solution was applied to the column until it was sunken in the column bed, so was $1.5 \mathrm{ml}$ of E1 buffer. After that $2.5 \mathrm{ml}$ of E1 buffer was applied and 13 fractions (200 $\mu \mathrm{l}$ per fraction) were eluted. UV-absorption spectroscopy and SDS-PAGE gel analysis were performed in order to confirm the success of ezrin labeling. 


\subsubsection{F-actin Polymerization}

In this work actin polymerization was performed in vitro to study the binding capacity of filamentous actin (F-actin) on solid supported and pore suspending membranes composed of DOEPC/DOPC (various composition) and on/within anodic aluminum oxide (AAO) substrates with different functionalizations. Either non muscular actin (> $95 \%$ purity, human platelet) composed of $85 \% \beta$-actin and $15 \% \gamma$-actin was used for experiments including ezrin or muscular actin (> $95 \%$ purity, rabbit skeletal muscle) consisting of pure $\alpha$-actin was utilized for all other experiments. Muscular as well as non muscular actin was purchased from Cytoskeleton (Denver, USA). Polymerization protocol was adapted from Bosk et al. and the manual provided by the cytoskeleton website. ${ }^{39,41}$

First, the lyophilized protein was aliquotted with cooled MilliQ water in $10 \mu \mathrm{l}$ portions with a concentration of $10 \mathrm{mg} / \mathrm{ml}$. Aliquots were stored at $-80^{\circ} \mathrm{C}$. For polymerization purposes one aliquot was dissolved in $238 \mu \mathrm{l} \mathrm{G}$ buffer to obtain a final globular actin (G-actin) concentration of $0.4 \mathrm{mg} / \mathrm{ml}(9.5 \mu \mathrm{M}, 1$ unit). Then $0.2 \mathrm{mM}$ ATP $(0.5 \mu \mathrm{l}$ of a $100 \mathrm{~mm}$ stock solution) and $0.5 \mathrm{mM}$ DTT ( $1.25 \mu \mathrm{l}$ of a $100 \mathrm{mM}$ stock solution) were added in order to reduce actin clustering and stabilize the globular actin monomer. Stock solutions of ATP and DTT were stored at $-80^{\circ} \mathrm{C}$ until usage. The mixture was incubated on ice for $1 \mathrm{~h}$, to allow for depolymerization of possible actin oligomers. The mixture was then centrifuged at $17000 \times \mathrm{g}$ at $4{ }^{\circ} \mathrm{C}$ for $15 \mathrm{~min}$ to pellet all remaining oligomers. After centrifugation, the supernatant was transferred in another Eppendorf ${ }^{\circledR}$ tube and polymerization was induced after addition of $\mathrm{F}$ salt solution $(25 \mu \mathrm{l}, 10 \%$ of the overall volume). After $20 \mathrm{~min}$ of polymerization $1 \mathrm{~mol} \%$ of the fluorescently labeled AlexaFluor488 phalloidin ( $5 \mu \mathrm{l}$ of a $6.6 \mu \mathrm{M}$ methanolic stock solution) was added to the polymerized actin and allowed to bind for $10 \mathrm{~min}$. Finally, the polymerization reaction was stopped by addition of $99 \mathrm{~mol} \%$ phalloidin ( $5 \mu \mathrm{l}$ of a $0.47 \mathrm{~mm}$ methanolic solution). Phalloidin binds 1:1 exclusively and with high affinity to F-actin, stabilizes the filaments and prevents depolymerization as well as further polymerization. ${ }^{42}$ It was as- 
sumed that after approximately $1.5 \mathrm{~h}$ all F-actin binding places were saturated with phalloidin. Hence, after this incubation time $0.5-1$ units of the F-actin solution was given to a solid supported or pore suspending membrane and to the functionalized AAO substrates respectively (final F-actin concentration $\sim 1 \mu \mathrm{M}$ ). The samples were incubated overnight at $4{ }^{\circ} \mathrm{C}$ in F-buffer to allow for F-actin binding on the corresponding surface.

G buffer: $\quad 0.2 \mathrm{mM} \mathrm{CaCl}_{2}, 0.2 \mathrm{mM} \mathrm{ATP}, 5 \%(w / v)$ sucrose, $1 \%(w / v)$ dextran, $5 \mathrm{mM}$ TRIS/HCl, $\mathrm{pH} 8$

F salt solution: $500 \mathrm{mM} \mathrm{KCl,} 20 \mathrm{mM} \mathrm{MgCl}$, $20 \mathrm{mM} \mathrm{ATP}$

F buffer: $\quad 50 \mathrm{mM} \mathrm{KCl}, 0.2 \mathrm{mM} \mathrm{MgCl}$, $0.1 \mathrm{mM}$ EDTA, $0.1 \mathrm{mM} \mathrm{NaN}, 20 \mathrm{mM}$ TRIS/HCl, pH 7.4

\subsection{Biophysical Methods}

\subsubsection{Impedance Spectroscopy}

Impedance spectroscopy (IS) is a versatile, non invasive technique to electrically characterize the properties of adherent cells, ${ }^{43}$ solid supported membranes ${ }^{44}$ and ion channels. ${ }^{45}$

In this work, IS was used to control, whether surface coverage of the self assembled monolayer (SAM) composed of octanethiol on the gold electrode of an AT-cut quartz crystal as well as the hybrid solid supported membrane formed after vesicle spreading, was sufficient.

\section{Physical Background}

Impedance spectroscopy is performed by applying an AC voltage $U(\omega, t)(\omega=$ angular frequency) with a small amplitude $\left(U_{0}\right)$ across the gold electrode-octanethiol-lipid sys- 
tem and measuring the corresponding current $I(\omega, t)$. The ratio $U(\omega, t) / I(\omega, t)$ defined as impedance $Z(\omega)$ as well as the phase shift $\varphi(\omega)$ between $U(\omega, t)$ and $\mathrm{I}(\omega, t)$ is recorded in dependence of the frequency $f$. The relationship between the mentioned values is specified in eq. 3.5.

$$
Z(\omega)=\frac{U(t, \omega)}{I(t, \omega)}=\frac{U_{0}}{I(t, \omega)} \cdot e^{i \varphi(\omega)}=|Z(\omega)| \cdot e^{i \varphi(\omega)} .
$$

Measured variables are the absolute value of impedance $|Z(\omega)|$ (eq. 3.6) and the phase shift $\varphi(\omega)$ described by eq. 3.7:

$$
\begin{array}{r}
|Z(\omega)|=\sqrt{\left(Z_{\text {real }}(\omega)\right)^{2}+\left(Z_{\text {im }}(\omega)\right)^{2}}, \\
\varphi(\omega)=\arctan \left(\frac{Z_{\text {im }}(\omega)}{Z_{\text {real }}(\omega)}\right),
\end{array}
$$

with the effective resistance $Z_{\text {real }}(\omega)$ and the reactive part of impedance $Z_{\text {im }}(\omega)$.

\section{Impedance Analysis of Solid Supported Membranes}

Solid supported mono- or bilayers obtain a specific resistance $\left(R_{\mathrm{m}}\right)$ and capacitance $\left(C_{\mathrm{m}}\right)$ that can be described in analogy to a plate type capacitor (eq. 3.8):

$$
C_{m}=\frac{\varepsilon_{0} \cdot \varepsilon_{r} \cdot A}{d},
$$


with $\varepsilon_{0}=8.854 \cdot 10^{-12} \mathrm{As} / \mathrm{Vm}, \varepsilon_{\mathrm{r}}$ being the relative permittivity ( $\varepsilon_{\mathrm{r}}$ of hydrophobic organic material $\simeq 2, \varepsilon_{\text {r }}$ of polar lipid head groups $\simeq 10-80$ ), $A$ as the mono- or bilayer surface and $d$ as the respective thickness.

Since a series of conductors add up reciprocally, the total membrane capacity is mainly determined by the hydrophobic part of the alkanethiol and lipid respectively. To completely electrically describe a solid supported membrane (Fig. $3.12 \mathrm{~A}$ ), an equivalent circuit can be used. The resistance of electrolyte and membrane ( $R_{\Omega}$ and $\left.R_{\mathrm{m}}\right)$ as well as membrane capacity $\left(C_{\mathrm{m}}\right)$ and the capacitance $\left(C_{\mathrm{el}}\right)$ at the electrode-electrolyte and membrane-electrolyte interface can be named as parts of equivalent circuit (Fig. 3.12 B). The circuit can be simplified under the following assumptions. First, $C_{\text {el }}$ is significantly higher than $C_{\mathrm{m}}$, which means that $C_{\mathrm{el}}$ can be neglected as the conductors are in series. Second, the high membrane resistance $R_{\mathrm{m}}$ is not detectable at the applied frequency range of $f=10^{-1}-10^{6} \mathrm{~Hz}$. Hence, the equivalent circuit can be reduced to an RC-circuit composed of $R_{\Omega}$ and $C_{\text {el+m }}$ (Fig. 3.12 C).
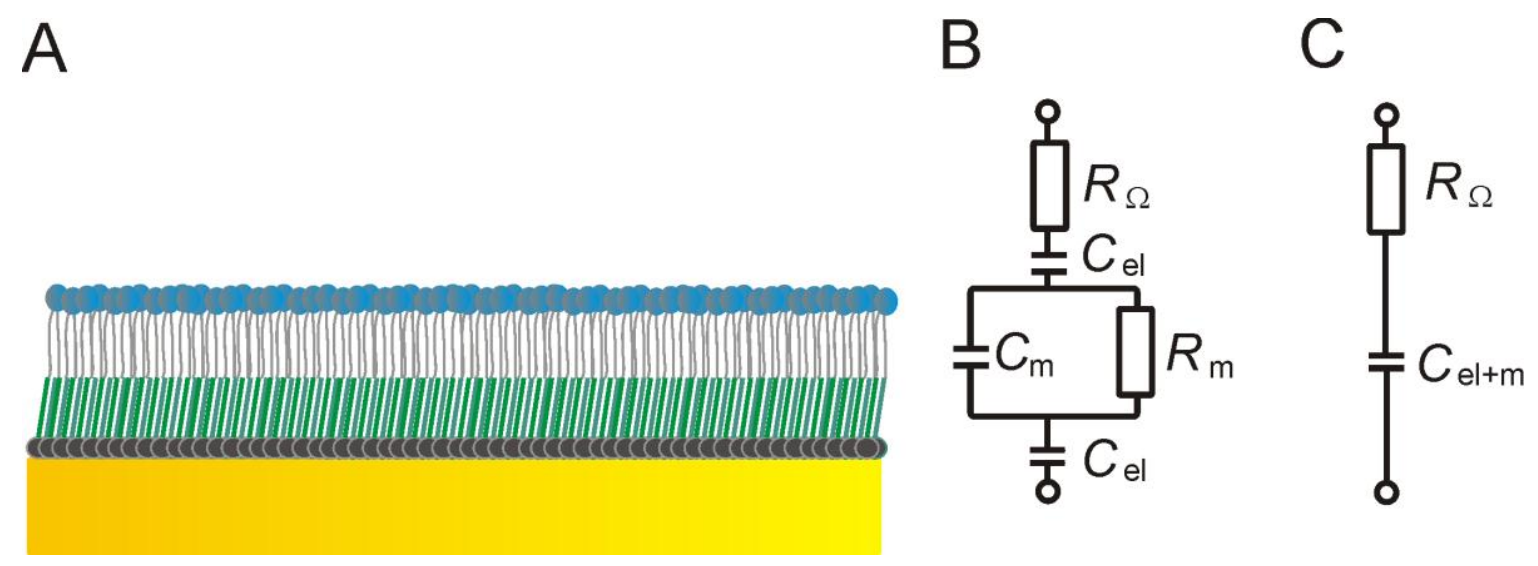

Fig. 3.12 (A) Solid supported hybrid membrane composed of an alkanethiol SAM chemisorbed on a gold electrode and a lipid monolayer. (B) Complete equivalent circuit that electrically characterizes the solid supported membrane. (C) Simplified equivalent circuit.

To evaluate impedance spectra the measured values $|Z(\omega)|$ and $\phi(\omega)$ are plotted in dependence of the frequency $\left(f=10^{-1}-10^{6} \mathrm{~Hz}\right)$ as a Bode-plot. A simulated Bode-plot 
with a capacitance $C_{\mathrm{el}+\mathrm{m}}=0.255 \mu \mathrm{F}$ and a resistance of $R_{\Omega}=200 \Omega$ is depicted in Fig. 3.13.

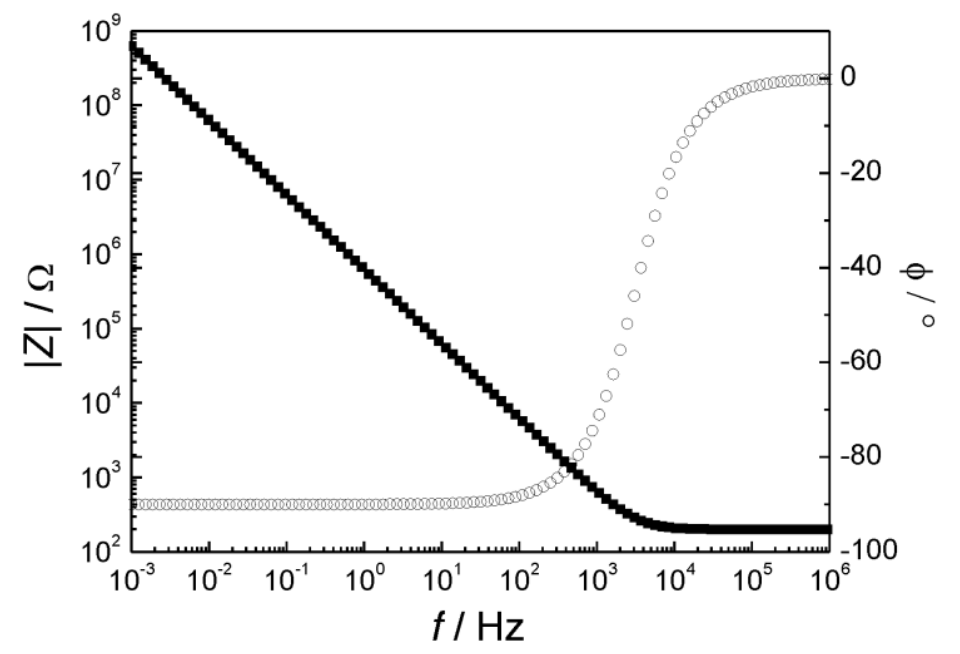

Fig. 3.13 Simulated Bode-plot of the phase shift $\varphi(\omega)$ (hollow circles) and impedance $|\mathrm{Z}(\omega)|$ (filled squares) as a function of frequency (range: $f=10^{-3}-10^{6} \mathrm{~Hz}$ ) with $C_{\mathrm{el}+\mathrm{m}}=0.255 \mu \mathrm{F}$ and $R_{\Omega}=200 \Omega$.

The Bode-plot shows that at high frequencies of $f=10^{4}-10^{6} \mathrm{~Hz},|\mathrm{Z}(\omega)|$ is mainly determined by the frequency independent resistance $\mathrm{R}_{\Omega}$ and the phase shift between $U(\omega, t)$ and $\mathrm{I}(\omega, t)$ is $\varphi(\omega)=0^{\circ}$, while at a frequency range of $f=10^{-3}-10^{4} \mathrm{~Hz}$ the membrane capacitance can be monitored in terms of an increase in $|\mathrm{Z}(\omega)|$ and a phase angle shift to $\varphi$ $(\omega)=90^{\circ}$.

\section{Experimental Setup and Data Evaluation}

Impedance spectroscopic analysis of both the octanethiol monolayer on the gold electrode and the solid supported hybrid bilayer were performed by means of the impedance analyzer SI 1260 from Solatron Instruments (Farnborough, UK) controlled by a personal computer. Working electrode was the gold electrode on top of the quartz crys- 
tal, the backing electrode was a platinum plated platinum wire that was dipped into the buffer solution covering the system (see Fig. 3.14).

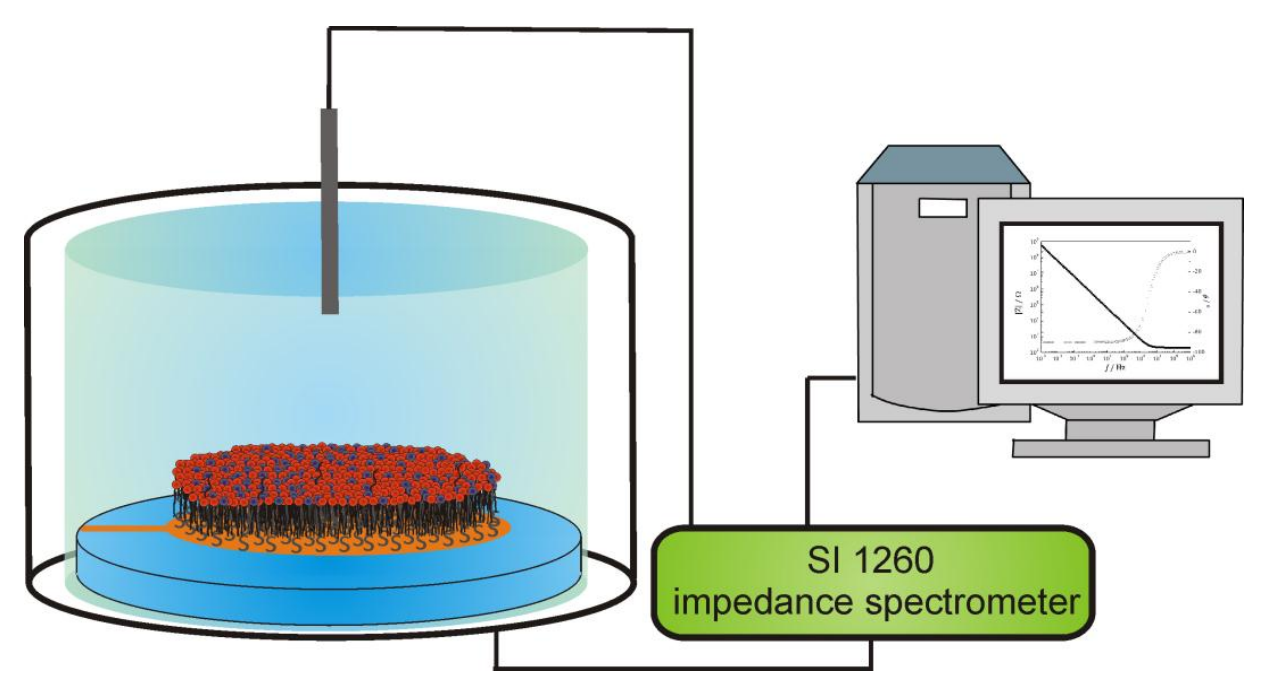

Fig. 3.14 Experimental setup for impedance spectroscopic measurements. The system is connected to an SI 1260 impedance spectrometer that is controlled by a personal computer via a working electrode (gold electrode on top of the quartz crystal) and a backing electrode (platinum plated platinum wire).

Spectra were recorded with 61 data points in a frequency range of $f=10^{-1}-10^{6} \mathrm{~Hz}$ with a small voltage amplitude of $30 \mathrm{mV}$ to prevent any membrane damage. A non linear square fit was used to extract the membrane specific parameters (eq. 3.9 and eq. 3.10).

$$
\begin{gathered}
|Z(\omega)|=\sqrt{R_{\Omega}{ }^{2}+\left(\frac{1}{\omega \cdot C_{e l+m}}\right)^{2}}, \\
\varphi(\omega)=\arctan \left(\frac{1}{\omega \cdot C_{e l+m} \cdot R_{\Omega}}\right) .
\end{gathered}
$$




\subsubsection{Circular Dichroism Spectroscopy}

Circular dichroism (CD) spectroscopy has become a versatile technique for examining the secondary and tertiary structure of proteins, folding/unfolding events and even for kinetic studies using the stopped flow technique. ${ }^{46}$

In this work, the secondary structure of the used proteins was studied by means of CD spectroscopy. Hence the success of protein purification and correct folding was confirmed. Additionally the influence of $\mathrm{Ca}^{2+}$ binding to the $\mathrm{C}$-terminal fragment of polycystin-2 (cPC2) on its secondary structure was investigated.

\section{Physical Background}

The effect of circular dichroism refers to the different absorption of left and right circular polarized light by optical active (chiral) compounds i.e. proteins. Linear polarized light can be viewed as two circularly polarized components with electrical field vectors rotating in opposite directions ( $E_{1}$ and $E_{r}$ ), but with the same amplitude (Fig. $3.15 \mathrm{~A}$ ). After passing through an optical active sample one of this component is absorbed to a greater extent. This results in elliptically polarized light, as one of the components amplitude is decreased (Fig. 3.15 B).
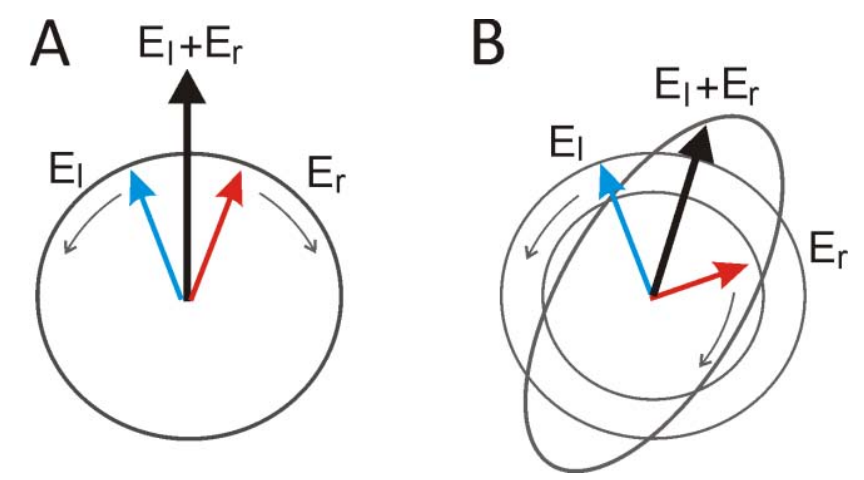

Fig. 3.15 (A) Electric field vectors of left (blue) and right (red) circularly polarized light. Superposition gives linear polarized light (black vector). (B) After passing an optical active sample that absorbs mostly right circularly polarized light elliptically polarized light arises. 
A CD spectrometer measures this effect as a difference in absorbance $(\triangle A)$ of the two circularly polarized components $\left(A_{\mathrm{L}}\right.$ as the absorbance of circular polarized light that rotates counter clockwise or clockwise $\left.A_{\mathrm{R}}\right)$, usually given as the ellipticity $\Theta$ in degrees (eq. 3.11).

$$
\Theta=32.98 \cdot \Delta A=32.98 \cdot\left(A_{\mathrm{L}}-A_{\mathrm{R}}\right) \text {. }
$$

By means of Beer-Lamberts law (eq. 3.4) eq. 3.11 can be further modified to eq. 3.12:

$$
\Theta=32.98 \cdot\left(\varepsilon_{\mathrm{L}}+\varepsilon_{\mathrm{R}}\right) \cdot d \cdot c=\Delta \varepsilon \cdot c \cdot l,
$$

with $\varepsilon_{\mathrm{L}}$ and $\varepsilon_{\mathrm{R}}$ being the extinction coefficients corresponding to $A_{\mathrm{L}}$ and $A_{\mathrm{R}}, c$ being the sample concentration and $l$ being the length of the cuvette.

In order to compare $C D$ spectra of different samples i.e. proteins the means residue ellipticity $\Theta_{M R}$ was established (eq. 3.13), where the CD signal is normalized to the number of amino acids $\left(n_{\mathrm{AA}}\right)$, protein concentration $\left(c_{\mathrm{P}}\right)$ and the length of the cuvette $(l)$.

$$
\Theta_{\mathrm{MR}}=\frac{\Theta[\mathrm{mdeg}]}{c_{P}[\mathrm{M}] \cdot l[\mathrm{~cm}] \cdot n_{\mathrm{AA}} \cdot 10}
$$




\section{Protein Structural Analysis}

Below $240 \mathrm{~nm}$, peptide bond absorption occurs (weak, but broad $n \rightarrow \pi^{*}$ transition at $220 \mathrm{~nm}$ and an intense $\pi \rightarrow \pi^{*}$ transition at $190 \mathrm{~nm}$ ). This far UV region mainly allows to determine the composition the protein's secondary structure elements. ${ }^{47} \mathrm{Far} C D$ spectra of different protein structural elements are depicted in Fig. 3.16. There are algorithms available to determine the content of secondary structure elements of a given protein CD spectrum. The online sever DICHROWEB allows to use a number of algorithms and datasets to compare CD spectra of proteins with a known crystal structure with the given spectra of unknown composition. ${ }^{48}$ In this work, the CDSSTR algorithm with a dataset that comprises the spectra of 49 soluble proteins with known crystal structure (reference set 7) was used to evaluate the content of secondary structure elements.

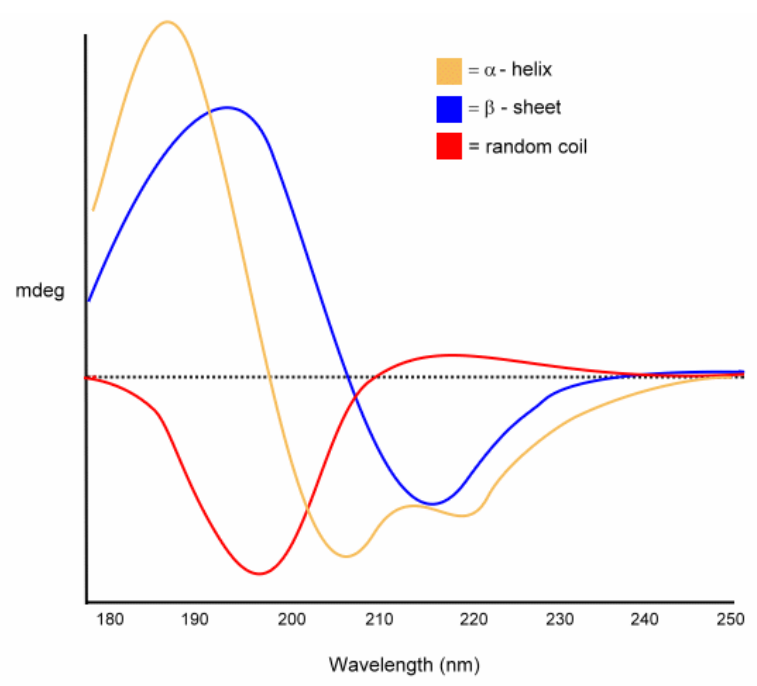

Fig. 3.16 Far UV CD spectra of different types of secondary structure elements: $\alpha$-helix (yellow), $\beta$-sheet (blue) and random coil (red). ${ }^{49}$

In order to analyze proteins in terms of their secondary structure, far UV spectra from 250-180 $\mathrm{nm}$ are required. To avoid high reference solution absorbance or scattering effects, some requirements have to be fulfilled. First, the sample should be free of scattering particles and the protein concentration has to be precisely determined and in a 
suitable range $(0.2-1 \mathrm{mg} / \mathrm{ml})$. Second, buffer components should be non-absorbing (avoid chloride ions, imidazole, Triton X-100). A good overview of components that are suitable for $\mathrm{CD}$ experiments is given by Kelly et al..46,50 In this work, a phosphate buffer was used for protein measurements. As some proteins were kept under high salt conditions in order to prevent aggregation and denaturation, they were rebuffered shortly before the experiment was conducted.

phosphate buffer: $\quad 20 \mathrm{mM} \mathrm{KH}_{2} \mathrm{PO}_{4} / \mathrm{K}_{2} \mathrm{HPO}_{4} 0.1 \mathrm{~mm}$ EDTA, pH 7.4

Experimental Setup and Performance

All CD experiments were performed using a Jasco-810 spectropolarimeter. The experimental setup is depicted in Fig. 3.17. A Xenon arc as light source gives a light output over the range of 178-1000 $\mathrm{nm}$. The light beam passes a monochromator and polarizer that produces linearly polarized light, which is equally composed of left and right circularly polarized light. A phase modulator then continuously switches the incident radiation between left and right circularly polarized component. After the light passes the sample, the absorbance ( $A_{\mathrm{L}}$ and $A_{\mathrm{R}}$ ) are detected by a photomultiplier. The signal is amplified and recorded by a personal computer. 


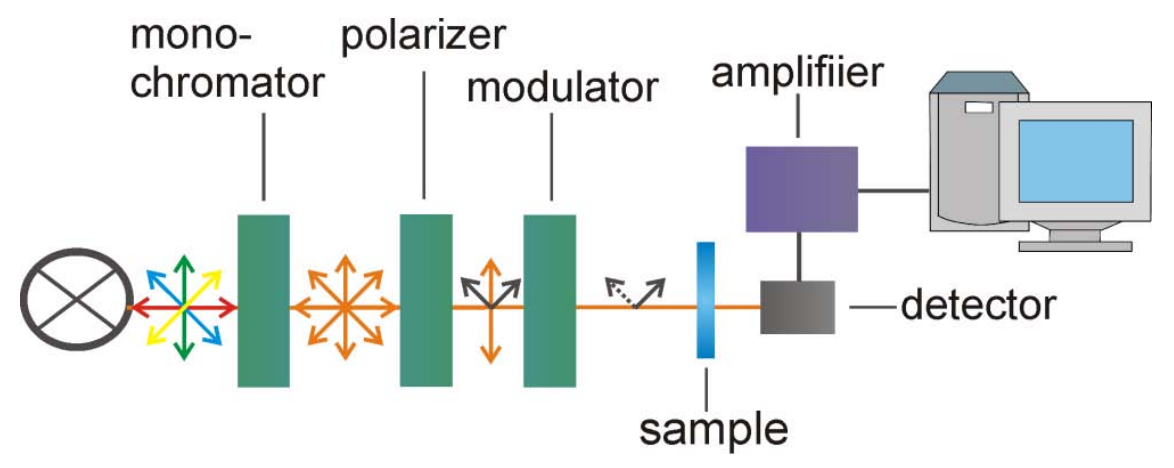

Fig. 3.17 Experimental setup of a CD spectropolarimeter. Two beams of monochromatic left and right circularly polarized light are provided by phase modulation (light source: xenon arc). The beams pass the sample and are detected by a photomultiplier. The signal is amplified and recorded by a personal computer.

The instrument was flushed with nitrogen before starting the experiment (20 min), because otherwise radiation of oxygen with $\lambda<200 \mathrm{~nm}$ would cause the formation of ozone that would damage the optical instruments. All measurements were performed with protein concentrations in the range of $2-6 \mu \mathrm{M}$ at $20^{\circ} \mathrm{C}$ in a wavelength range of $270-180 \mathrm{~nm}$ in a quartz glass cuvette $(l=0.1 \mathrm{~cm}$, minimum sample volume $=200 \mu \mathrm{l})$ with a scan speed of $20 \mathrm{~nm} / \mathrm{min}$ (averaged over seven spectra). A blank spectrum (only buffer inside the cuvette) was subtracted from the spectrum of the protein sample. The subtracted spectrum was then smoothed with a software provided by Jasco- 810 .

\subsubsection{Quartz Crystal Microbalance Technique}

Quartz resonators have gained popularity in electronic devices and analytical chemistry since the linear relationship between their frequency response and the loaded mass has been found by Sauerbrey in 1959.51,52 Since then, the quartz crystal microbalance (QCM) has been employed as an acoustic wave sensor for measuring particle deposition or thin film thickness in vacuum with a sensitivity in the range of $1 \mathrm{ng}$. However, it was only after appropriate oscillation circuits in fluids had been developed in the late 1980 's that the QCM was established as a versatile biosensor technique. ${ }^{53-55}$ Since then, numerous groups have studied adsorption processes of proteins, DNA hybridization, 
antigen antibody interactions or cell adhesion in a time resolved, label free manner. ${ }^{56-}$ 61,54

In this work, the QCM technique was used to monitor the binding event of the Cterminal domain of polycystin-2 (cPC2) with C-terminal polycystin-1 and PIGEA14 (polycystin-2 interactor, Golgi- and endoplasmic reticulum-associated protein with a mass of $14 \mathrm{kDa}$ ). These interactions were quantified in terms of their thermodynamics and kinetics as a function of $\mathrm{Ca}^{2+}$ concentration and pseudophosphorylation, respectively (see chapter 5 and 6).

\section{Physical Background}

The principle of QCM measurements is based on the piezoelectric behavior of the quartz crystal. The piezoelectric effect has first been described by Pierre and Jaques Currie in 1880. It is basically defined as an asymmetric charge accumulation and hence the generation of an electrical field when a material that lacks an inversion centre is under mechanical stress. As for quartz $\left(\mathrm{SiO}_{2}\right)$ a network composed of $\mathrm{SiO}_{4}$ tetrahedrons (Fig. $3.18 \mathrm{~A}$ ) that is put under mechanical stress leads to dislocation of negative and positive charges in the opposite direction and thus an electrical field originates (Fig. $3.18 \mathrm{~B})$. 

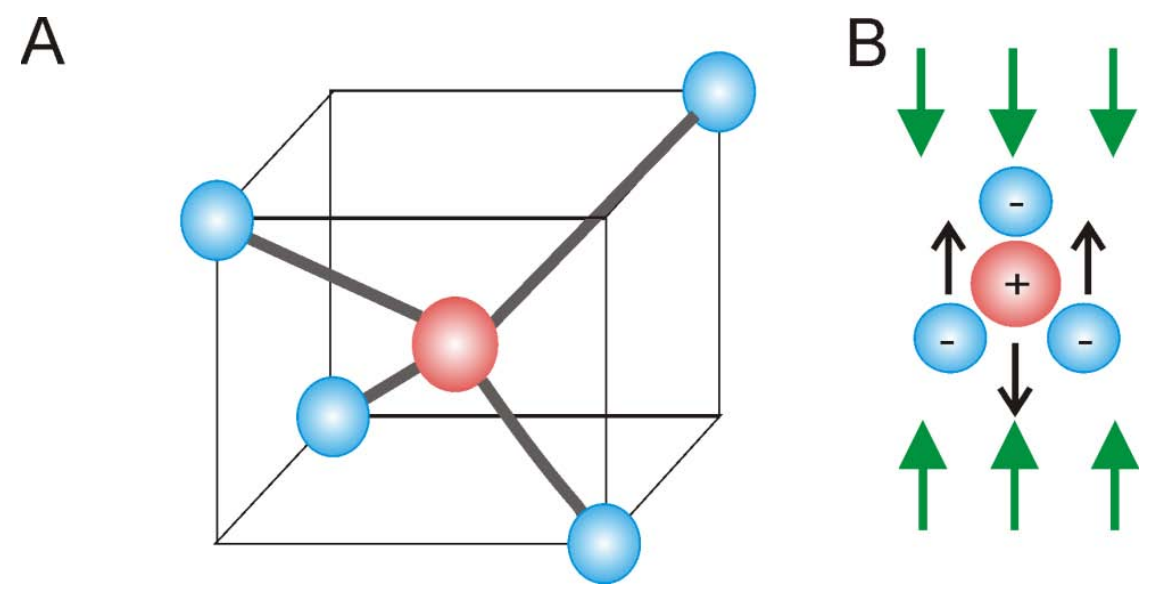

Fig. 3.18 (A) $\mathrm{A} \mathrm{SiO}_{4}$ tetrahedron as the smallest unit of the $\mathrm{Sio}_{2}$ network of quartz. (B) Side view of the tetrahedron shows the shift of positive (silicon) and negative (oxygen) charges under pressure.

Basis for the QCM technique is the so called converse piezoelectric effect, where the quartz crystal is deformed after applying an oscillating electrical field.

Due to the electrical field several deformations to the quartz crystal can be caused depending on the cut angle in relation to the crystal lattice. Thickness shear mode resonators (TSM resonators) consist of a quartz crystal with gold electrodes on each side (see Fig. 3.6 ) that generates bulk acoustic waves (BAW) travelling perpendicular to the particle displacement and sensor surface respectively, after being excited by an external stimuli i.e. an electrical potential (Fig. 3.19 B) ${ }^{62}$ Constructive interference occurs, if the acoustic wavelength $\left(\lambda_{\mathrm{Q}}\right)$ is an odd multiple of twice the crystal thickness $\left(d_{\mathrm{Q}}\right)$. This, standing waves as seen in Fig. $3.19 \mathrm{~B}$ are generated. The eigenfrequency $\left(f_{0}\right)$ of the quartz can be expressed by eq. 3.14 :

$$
f_{0}=\frac{v_{0}}{\lambda_{Q}}=\frac{v_{0}}{2 d_{Q}}
$$

The most common used TSM resonator is an AT-cut quartz crystal, which refers to a cut angle of $35.15^{\circ}$ to the optical z-axis of the crystal (Fig. 3.19 A). They have a temper- 
ature coefficient of almost zero in the range of $0-50{ }^{\circ} \mathrm{C}$ and a frequency stability of $\Delta f / f=10^{-8}$, which makes them suitable for highly sensitive biosensor applications. ${ }^{60,63}$ If a rigid mass is applied on top of the quartz surface the eigenfrequency decreases (see Fig. 3.19 B, red line).

A

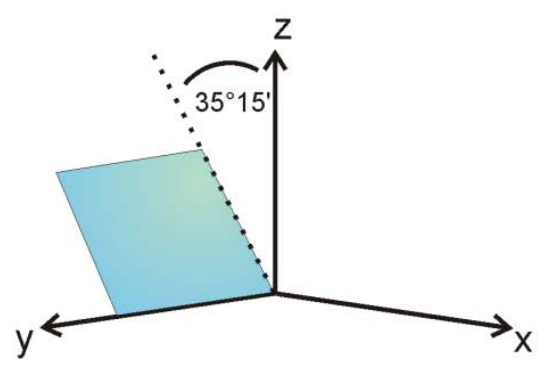

B

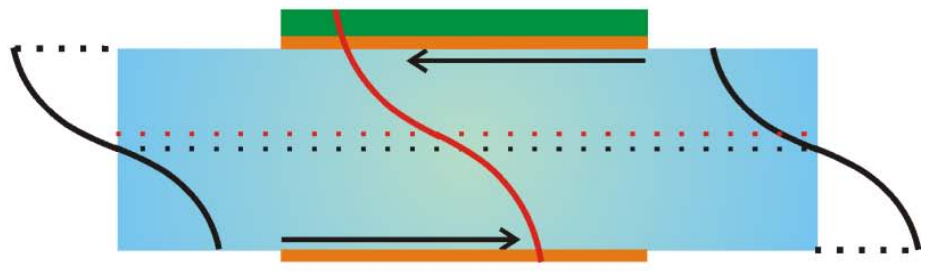

Fig. 3.19 (A) AT-cut quartz crystal. (B) Thickness shear mode (TMS) resonator with longitudinal particle displacement (back arrows) and transversal bulk acoustic waves (BAW, back lines). The BAW frequency is shifted to lower frequencies (red line), if a rigid mass (green) is applied on top of the gold electrode.

The linear relationship between the applied mass $(\Delta m)$ and the frequency shift $(\Delta f)$ has first been described by Sauerbrey et al. by eq. $3.15:^{51}$

$$
\Delta f=-\frac{2 f_{0}^{2} \Delta m}{A \sqrt{\mu_{Q} \rho_{Q}}}=-S \cdot \Delta m
$$

with $A$ being the surface area of the gold electrode $\left(A=0,255 \mathrm{~cm}^{2}\right)$, $\mu_{\mathrm{Q}}$ the shear modulus of the quartz $\left(\mu_{\mathrm{Q}}=29.47 \mathrm{GPa}\right), \rho_{\mathrm{Q}}$ the quartz density $\left(\rho_{\mathrm{Q}}=2.65 \mathrm{~g} / \mathrm{cm}^{3}\right)$ and $S$ being the Sauerbrey constant that denotes the integral mass sensitivity $(S=0.14 \mathrm{~Hz} / \mathrm{ng})$.

However, this relationship is only valid for an applied rigid mass in vacuum that has no more than $0.5 \%$ of the quartz mass and equals the quartz density. Most biosensor devises are used in a liquid environment, where the acoustic wave decays inside the 
liquid caused by damping of the viscous surrounding. ${ }^{64,65}$ Also for thick layers and elastic solids of viscoelastic films the Sauerbrey rule is no longer valid. Therefore, an extension of the equivalent circuit that describes an unperturbed resonator by additional elements is needed. The decay length of a $5 \mathrm{MHz} A T$-cut quartz at $20^{\circ} \mathrm{C}$ as used in this work is about $250 \mathrm{~nm}$.

\section{Electrochemical Coupling}

To describe the effect of mass load to an oscillating quartz crystal a mechanical model depicted in Fig. 3.20 B can be used. Within this mode, the mass $m$ is moved by an external force $F$ that provokes oscillation (spring is given with a spring constant Sauerbrey constant). Damping losses during oscillation are represented by the dashpot $r$. This mechanical mode can be transformed into an electrical model, the Butterworthvan-Dyke (BVD) equivalent circuit. This circuit contains a motional branch with a capacitance $(C)$, inductance $(L)$ and resistance $(R)$ in series. An additional capacitance $\left(C_{0}\right)$ is connected parallel to the series circuit in the static branch (Fig. 3.20 A).
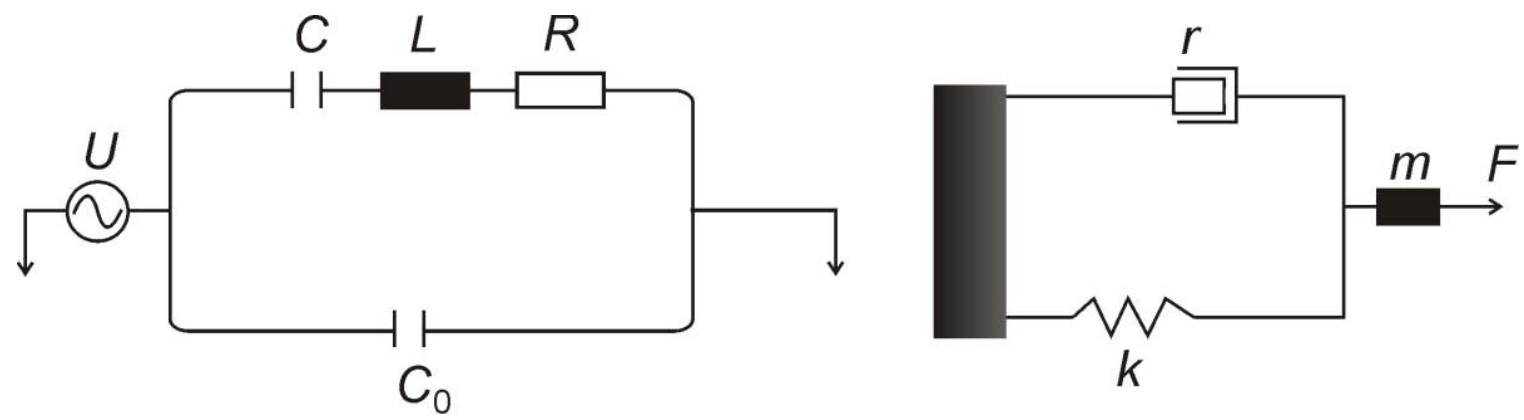

Fig. 3.20 (A) Butterworth-van-Dyke (BVD) equivalent circuit with lumped impedance elements. The circuit can be divided in a motional branch with a capacitance $(C)$, an inductance $(L)$ and a resistance (R) and a statical branch with a capacitance $C_{0}$ that represents the admittance away from resonance. (B) Mechanical model of a damped oscillator with the oscillating mass $(m)$, the dashpot $(\mathrm{r})$, the spring $(k)$.

Comparing the electrical and the mechanical model, the oscillating mass $m$ corresponds to the inductance $L$, the resistance $R$ can be compared with the dashpot $r$ and the spring 
$k$ corresponds to the conductivity $C$. The static capacitance $C_{0}$ is determined by the dielectric properties of the resonator that behaves like a capacitor far away from its resonance frequency. The altered voltage $U$ is equivalent to the external force $F$ of the mechanical model.

\section{QCM200}

As mentioned above the linear behavior between the frequency response $(\Delta f)$ is only linearly correlated to the applied mass $(\Delta m)$, if the mass is non viscoelastic. If a mass with a certain viscoelastic content adsorbs, energy of the quartz oscillation dissipates into the system, as a result of damping by the viscoelastic, adsorbed material. To assign the frequency shift during an adsorption process to the deposition of rigid mass a viscoelastic behavior has to be excluded. Hence, the amplitude controlled QCM200 from Stanford Research Systems Inc. (Sunnyvale, USA) was used to simultaneously monitor the shift of resonance frequency $(\Delta f)$ and the change of dynamical resistance $(\Delta R)$.

A shift of resonance frequency that is directly related to the applied rigid mass can be deduced from the shift of inductivity $(\Delta L)$, while the dynamical resistance $R$ is a measured variable that allows to follow and interpret the viscoelastic content of the adsorbed material. To correctly assign the respective measured variable, series resonance of the oscillator circuit has to be set and the series capacity $(C)$, which accounts for the elastic behavior of the quartz material, has to be assumed as constant. Therefore, the static $C_{\mathrm{o}}$ has to be manually compensated ("nulled") by a variable capacitor $\left(C_{\mathrm{v}}\right)$ parallel to Co. To stabilize the oscillation of the quartz in case of damping, the QCM200 includes an automatic gain control amplifier that stabilizes the quartz oscillation in case of damping.

\section{Experimental setup and performance}

QCM experiments were performed according to the experimental setup depicted in Fig. 3.21. First, a solid supported hybrid bilayer composed of octanethiol-lipid mono- 
layer (preparation described in chapter 3.1.2 and 3.2.3) was prepared on top of the gold electrode of an AT-cut quartz crystal that was placed inside a Teflon chamber. After controlling the quality of the obtained mono- and bilayer by means of impedance spectroscopy (see chapter 3.4.1), the Teflon chamber was placed inside a water jacketed Faraday cage $\left(\mathrm{T}=20^{\circ} \mathrm{C}\right)$ and connected to a flow through unit via an in- and outlet. It was made sure that no air bubbles were left inside the tubing, as they would destroy the membrane during an experiment. Protein or buffer solution (as chosen via the selection valve) was pumped with a constant rate of $0.39 \mathrm{ml} / \mathrm{min}$ to the sensor surface (stagnation flow point geometry) via a peristaltic pump. During an adsorption process a closed circuit was generated to avoid loss of protein material to the waste. Frequency shift $(\Delta f)$ and changes in dynamic resistance $(\Delta R)$ were recorded in a time resolved manner by the amplitude controlled QCM200 and monitored by a personal computer.

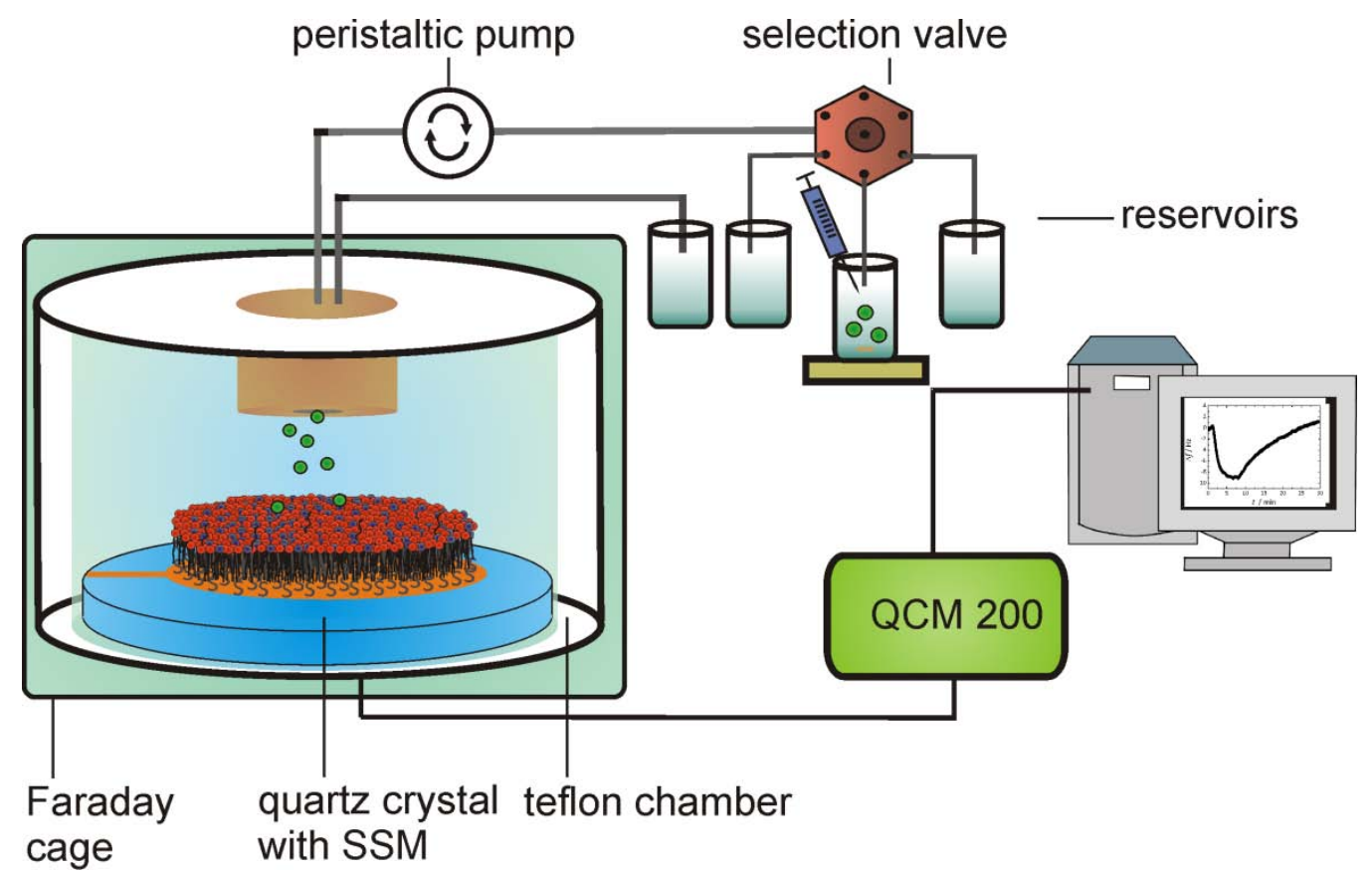

Fig. 3.21 Schematic drawing of the setup of a QCM experiment. 


\subsubsection{Kinetic Data Modeling}

Reversible adsorption kinetics of small particles such as proteins on a homogenous surface can be generally described by the Langmuir model (eq. 3.16):

$$
\frac{d \theta}{d t}=k_{\mathrm{on}} \pi a^{2} \rho_{\infty}(1-\theta)-k_{\mathrm{off}} \theta,
$$

with $k_{\text {on }}$ and $k_{\text {off }}$ being the rate constants of particle adsorption and desorption, respectively. $a$ is the particle radius, $\Theta$ the surface coverage, and $\rho_{\infty}$ is the density of the particles in bulk solution.

The model is based on the assumption that particle adsorption occurs noncooperatively on a homogeneous surface in a monomolecular fashion, where all individual binding sites have the same adsorption energy for the adsorbate. However, this model misses certain aspects of a realistic protein adsorption on a surface. First, the protein area covering one binding site can expand over more than one lattice space, which needs to be taken into account by assuming an appropriate available surface function $\Phi(\Theta) . \Phi(\Theta)$ describes the available surface for deposition of particles from bulk solution as a function of coverage. For the classical Langmuir kinetics $\Phi(\Theta)=1-\Theta$. Second, the density of the particles in bulk solution $\rho_{\infty}$ is different from the particle density near the surface $\rho(\delta)$. The flux $j$ of the particles to the surface, given by eq. 3.17, with the transport rate constant $k_{\mathrm{tr}}$, accounts for the transport of the particles to the surface:

$$
\frac{d \theta}{d t}=j=k_{t r}\left(\rho_{\infty}-\rho(\delta)\right)
$$


These two refinements lead to the generalized Langmuir-equation (eq. 3.18):

$$
\frac{d \theta}{d t}=k_{\mathrm{on}} \pi a^{2} \rho(\delta) \Phi(\theta)-k_{\mathrm{off}} \theta
$$

Combining eq. 3.17 and eq. 3.18 gives eq. 3.19:

$$
\frac{d \theta}{d t}=\frac{k_{\mathrm{on}} \pi a^{2} \rho_{\infty} \Phi(\theta)-k_{\mathrm{off}} \theta}{1+\frac{k_{\mathrm{on}}}{k_{\mathrm{tr}}} \Phi(\theta)} .
$$

As the available surface function $\Phi(\Theta)$ cannot be given in a compact analytical expression, there are different strategies (i.e. developing in a virial series) that can be pursued. The scaled particle theory (SPT) is an approach to calculate the available surface function, which provides accurate results even at high coverage (eq. 3.20):

$$
\Phi(\theta)=(1-\theta) \exp \left[-3 \frac{\theta}{1-\theta}-\left(\frac{\theta}{1-\theta}\right)^{2}\right]
$$

SPT approximates the work of cavity formation in a hard-sphere fluid, which might be a submonolayer of spheres covering a surface, exactly for cavities smaller than a solvent particle. Reiss et al. ${ }^{66}$ developed an interpolation based on a number of exact conditions imposed by geometry and thermodynamics that spans from small cavity radii to the limit of macroscopic size providing an equation of state. It has been shown that this 
approach correlates with data derived from dynamic Monte Carlo simulations up to a surface coverage near jamming limit. ${ }^{67,68}$

For kinetic fitting using eq. 3.19 we developed a Matlab routine based on a downhill simplex method (Nelder-Mead algorithm) combined with a built in Dormand-Prince solver (ODE 45) to integrate eq. 4.

\subsubsection{Surface Plasmon Resonance Spectroscopy}

Affinity based optical biosensors have gained significant popularity to monitor analyteligand interactions in real time in order to investigate the kinetics and thermodynamics of the binding processes. Optical techniques that detect changes of the refractive index/thickness at a solid/water interface are either reflection based such as ellipsometry, interference based i.e. reflectometric interference spectroscopy (RIfS) or evanescent wave techniques as surface plasmon resonance (SPR), optical waveguide spectroscopy (OWS) and attenuated total reflection infrared (ATR-IR) spectroscopy. By means of these techniques surface coupled binding processes can be analyzed in a label-free manner with the advantage that no artificial fluorescent labels that may interfere with the function of the molecules are needed to investigate the respective interaction. ${ }^{69-71} \mathrm{~A}$ grown number of commercially available evanescent wave techniques indicate that they exhibit distinct properties that qualify them as superior compared to the others mentioned above. First, a high sensitivity (refractive index changes of $\Delta n / n=10^{-5}$ ) can be achieved due to the evanescent field coupling at the interface of a dielectric medium and a noble metal. Second, small sample volumes can be employed as both excitation and detection take place at the opposite side of the flow cell machinery (see Fig. 3.26).

In this work, both SPR and OWS are used to investigate protein-membrane and protein-protein interactions in a label-free and time resolved manner. In case of the SPR experiments, planar gold coupled solid supported hybrid membranes were used to specifically immobilizes proteins that serve as ligands to investigate their binding capability to other proteins (for sample preparation see chapter 3.1.2 and 3.2.3). OWS ex- 
periments were performed to investigate interactions that exclusively take place inside the pores of porous anodic aluminum oxide substrates that were functionalized with a solid supported hybrid membrane prior to the experiments (preparation described in chapter 3.1.2 and 3.2.3).

\section{Physical Background}

Surface plasmons or surface polaritons are charge density oscillations of an electromagnetic wave that propagates parallel (along the $\mathrm{x}$-axis) to a noble metal/dielectric interface. For many reasons such as chemical resistance and capability of surface modification gold is the most frequently used noble metal, while silver, copper and indium are other alternatives. The penetration depth (z-direction) of the excited surface plasmons decays exponentially (so called evanescent field) into the dielectric. This confines the sensitivity to $\sim 250 \mathrm{~nm}$ from the sensor surface. Excitation of these plasmons can be achieved by an evanescent wave that exponentially decays into the noble metal (gold layer thickness restricted to $45-55 \mathrm{~nm}$ ) after total internal reflection (TIR: all light is reflected, no light is refracted inside the media) of an incoming light beam at the metal interface (Fig. 3.22). 


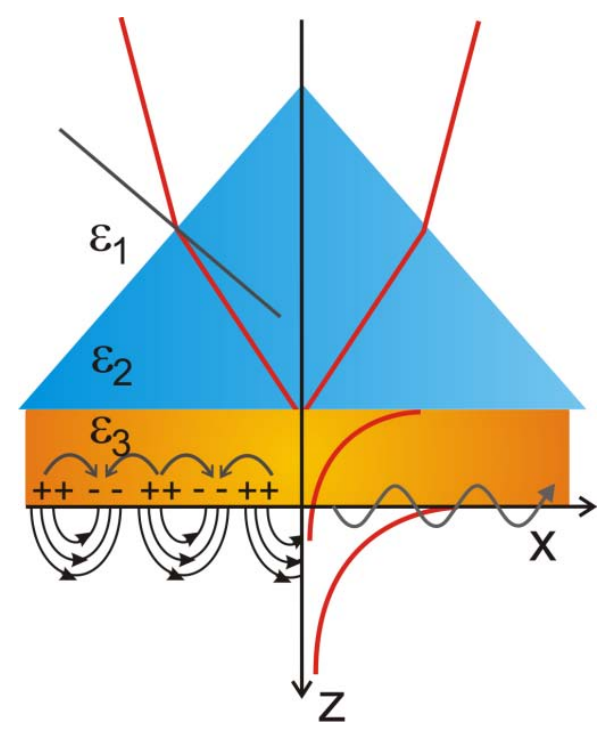

Fig. 3.22 Surface Plasmons (left: charge density oscillation, right: electromagnetic wave) that are excited at a gold/dielectric interface by an evanescent wave after total internal reflection at the prism/metal interface. The reflection and refraction a of a light beam (red) that hits a medium with a dielectric constant $\left(\varepsilon_{1}>\varepsilon_{2}, \varepsilon_{3}>\varepsilon_{2}\right)$ takes place according to Snell's law.

According to Snell's law (eq. 3.21), the phenomenon of total internal relfection (TIR) only occurs, if light travels from a medium with higher a refractive index or dielectric constant $\left(\varepsilon_{i}=\varepsilon_{2}\right)$ to one with a lower refractive index (Fig. 3.22, $\varepsilon_{m}=\varepsilon_{3}$ ). Otherwise, the refracted light beam gets closer to the normal,

$$
\frac{\sin \left(\theta_{\mathrm{i}}\right)}{\sin \left(\theta_{\mathrm{m}}\right)}=\sqrt{\frac{\varepsilon_{\mathrm{m}}}{\varepsilon_{\mathrm{i}}}},
$$

with $\Theta_{\mathrm{i}}$ and $\Theta_{\mathrm{m}}$ as the angle measured from the interface normal and the incoming and refracted light. The dielectric constants $\varepsilon_{\mathrm{i}}$ and $\varepsilon_{\mathrm{m}}$ of the corresponding medium with a refractive index of $n=\sqrt{\varepsilon}$. The subscripts $\mathrm{m}$ and i can be integral numbers that characterize different dielectric properties of the respective media.

As surface plasmons propagate along the metal/dielectric interface ( $\mathrm{x}$-direction) only light with an electromagnetic field component in $\mathrm{x}$-direction is capable of plasmon exci- 
tation. While light with an electrical field vector $(\vec{E})$ that propagates in the plane of incident (p-polarized light or transverse magnetic (TM) polarized light, $\vec{E}_{T M}=\left(E_{x,}, 0, E_{z}\right)$ ) can constructively be coupled into the gold, s-polarized or transverse electric (TE, $\left.\vec{E}_{T E}=\left(0, E_{y,}, 0\right)\right)$ polarized light cannot. ${ }^{72}$ More precisely, the moment of the photon in x-direction $\left(\vec{k}_{x}\right)$ must match with the momentum of the plasmon $\left(\vec{k}_{S P}\right)$, so that surface plasmon resonance can occur. According to eq. $3.22 \vec{k}_{x}$ is directly related to the angle of incident $\left(\Theta_{\mathrm{i}}\right)$ of the incoming light as depicted in Fig. 3.23. A higher angle of incident means a greater value of $\vec{k}_{x}$,

$$
\vec{k}_{x}=\vec{k} \cdot \sin \theta_{i}
$$

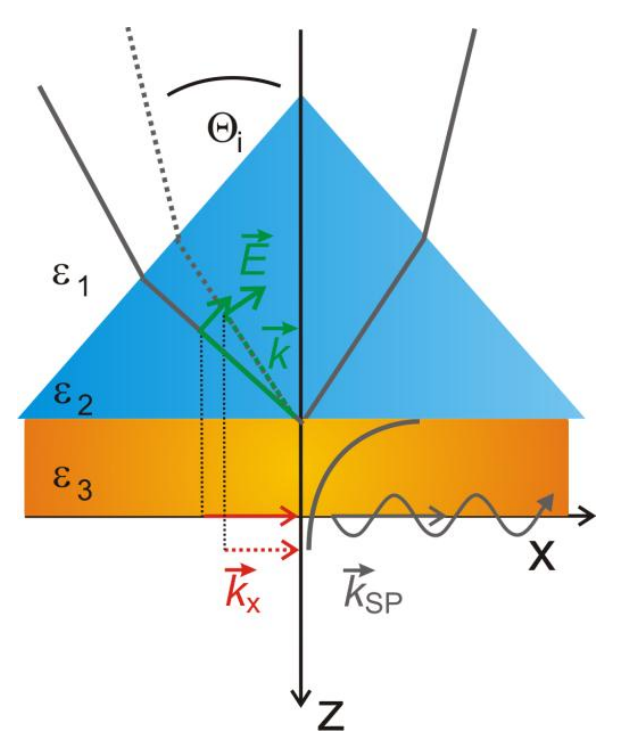

Fig. 3.23 Surface plasmon excitation via p-polarized light with an electromagnetic wave vector $(\overrightarrow{\boldsymbol{E}})$ propagating in the plane of incident. The value of the momentum in x-direction $\left(\overrightarrow{\boldsymbol{k}}_{\mathbf{x}}\right)$ is increased by increasing the angle of incident until it matches the momentum of the plasmon $\left(\overrightarrow{\boldsymbol{k}}_{\mathbf{s p}}\right)$. Dielectric constants correspond to the respective media: $1=$ air, $2=$ prism and $3=$ gold . 
However, without prism coupling it is impossible to achieve matching conditions of the two wave vectors $\vec{k}_{s p}$ and $\vec{k}_{\mathrm{x}}$. This fact can be explained by comparing the dispersion relation of the plasmon that propagates along the gold interface ( $\varepsilon_{1}$ and $\varepsilon_{3}$, eq. 3.23) and the one of the photon $\left(\vec{k}_{\mathrm{ph}}\right)$ that propagates in a medium with the dielectric constant $\varepsilon 1$ respectively (eq. 3.24)

$$
\begin{array}{r}
\omega=\vec{k}_{x} \cdot c \cdot \sqrt{\frac{1}{\varepsilon_{1}}+\frac{1}{\varepsilon_{3}}}, \\
\omega=\frac{\vec{k}_{x}}{\sin \theta} \cdot c \cdot \sqrt{\frac{1}{\varepsilon_{1}}},
\end{array}
$$

with the angular frequency $(\omega)$, velocity of light (c) and dielectric constants that correspond to those described in Fig. 3.23 .

As depicted in Fig. 3.24 A under non coupling conditions the tangent line (red) representing the dispersion relation of the photon in medium 1 (air) would not intersect with the curve (grey) that results from the dispersion relation of the plasmon at the gold/air interface (medium 1 and 3). Only by using a high refractive index prism (medium 2, with $\varepsilon_{2}>\varepsilon_{1}$ ) as coupling device, the slope of the photons dispersion relation can be decreased in a way that it intersects with the plasmon curve (blue). 

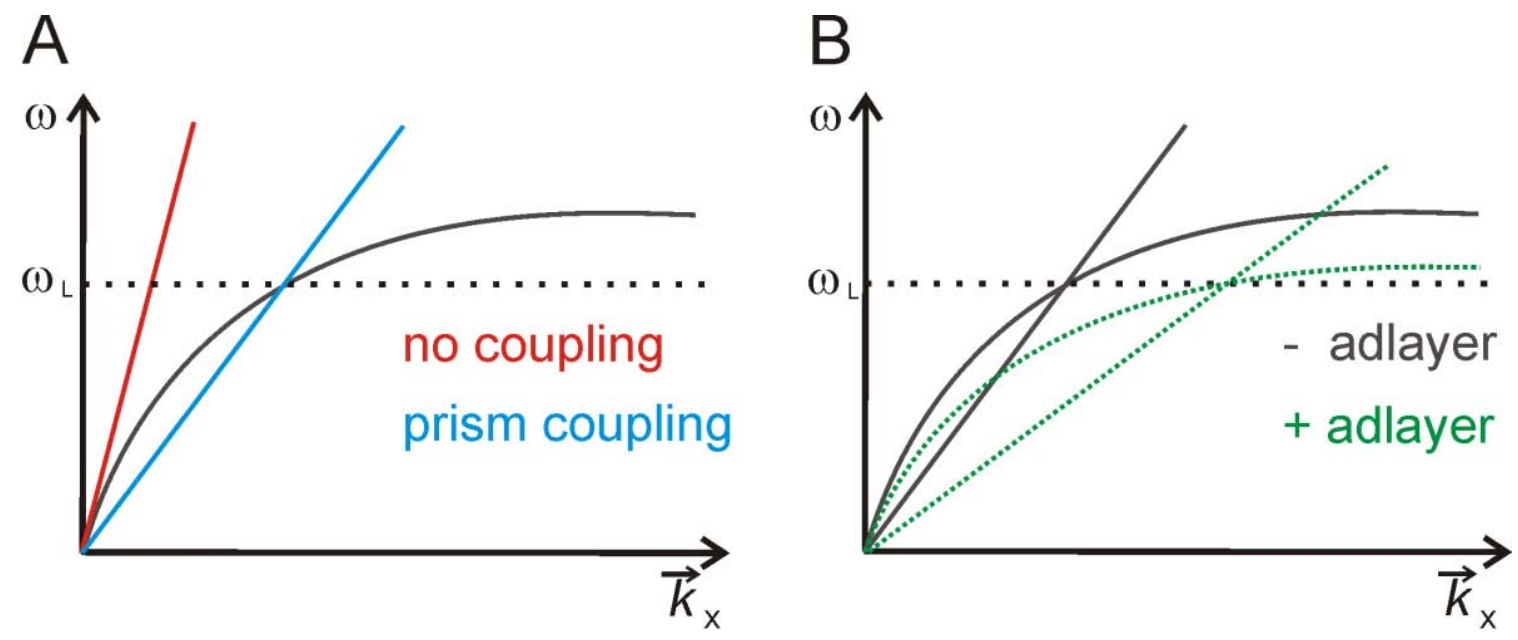

Fig. 3.24 (A) Dispersion relation of a photon that propagates in air $(\varepsilon 1$, red). With a high refractive index prism as coupling device ( $\varepsilon_{2}$, blue) the curves of the photon and the plasmon (grey curve) that propagates along a gold/air interface ( $\varepsilon_{3}$ and $\varepsilon_{1}$ ) intersect. (B) Dispersion relation of interacting photon and plasmon dispersion relation curves without a dielectric adlayer at the metal interface (straight grey curves) and with an adlayer (dotted green curves). While $\omega$ represents the angular frequency ( $\omega \mathrm{L}=$ angular laser frequency) $\overrightarrow{\boldsymbol{k}}_{\mathbf{x}}$ represent the wavevector of the plasmon and photon in x-direction.

An adlayer on the gold surface (i.e. after ligand or analyte adsorption) would damp the surface plasmon and would cause a shift in dispersion relation as depicted in Fig. 3.24 B, (dotted line) Hence, the slope of the photon dispersion relation has to be further decreased (wave vector $\vec{k}_{x}$ has to be increased) by increasing the angle of incident $\left(\Theta_{\mathrm{i}}\right)$ of the incoming light according to eq. 3.24. The shift of $\Theta_{i}(\Delta \Theta)$ is proportional to the adlayer thickness $(d)$ and the refractive index change $(n)$.

There are two possible configurations in order fulfill the prism's coupling condition. First, the Otto configuration is assembled in a way that there is a small gap ( $2000 \mathrm{~nm})$ between the prism and a thick metal layer, where adsorption processes take place. This experimental setup is quite complicated, which is why the so called Kretschmann setup has been developed later. In the Kretschmann configuration the evanescent wave propagates through a thin gold layer $(45-55 \mathrm{~nm})$ and excites the plasmon a the opposite site. 

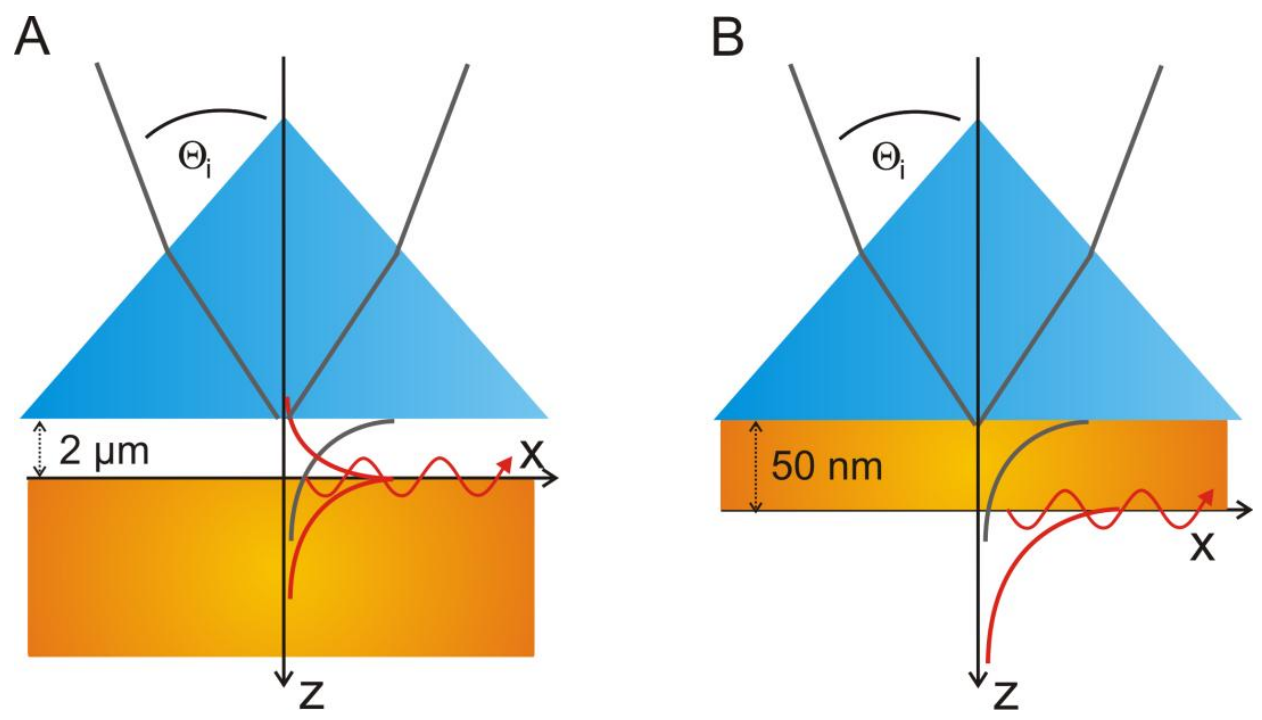

Fig. 3.25 Schematic drawing of the Otto (A) and Kretschmann (B) SPR-configuration.

Experimental Setup and Performance

SPR experiments were performed using a Res-TEC2005 spectrometer with the experimental setup depicted in Fig. 3.26. ${ }^{73}$ Light source was a HeNe-laser that emits monochromatic light with a wavelength of $\lambda=632.8 \mathrm{~nm}$. The light beam is first reflected by two mirrors, then passes a beam chopper, two polarizer and a shutter until it hits the LaSFN9 high refractive index prism $(n=1.84)$. The first polarizer is used to adjust the light intensity (intensity was given in Volts by a photon counter); the second polarizer is used to create p-polarized light. The reflected light hits a photodiode that transmits the light signal to a photon counter, which transforms the light signal in an electrical signal (in Volts) and further to a personal computer (WASPLAS software). The prism is connected with the non gold coated side of a LaSFN9 glass plate via high refractive index immersion oil. The gold coated functionalized side is facing a flow cell assembly with in- and outlet tubings that are connected with a flow through system driven by a peristaltic pump. 


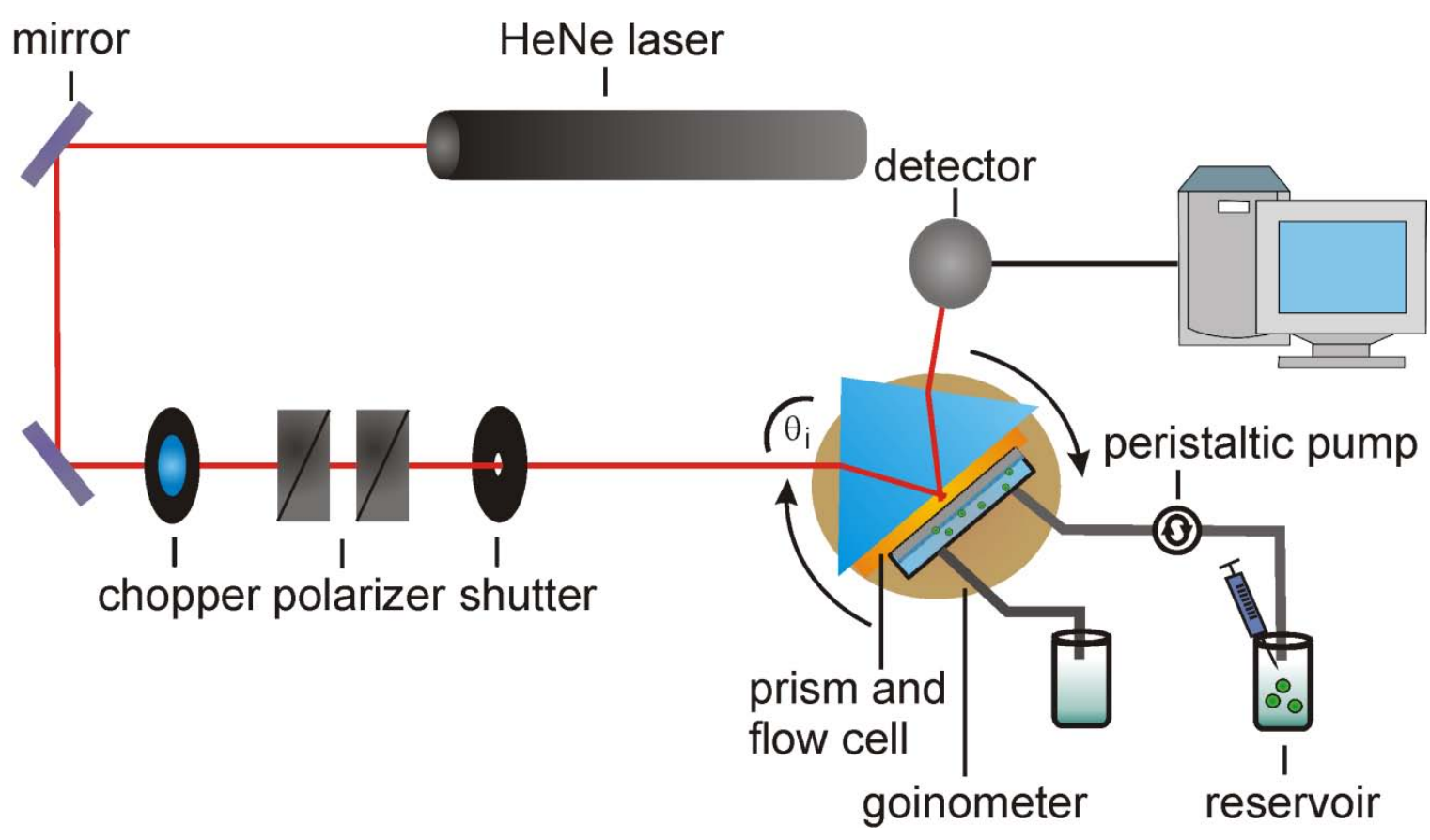

Fig. 3.26 Schematic drawing of the SPR and OWS experimental setup.

Before starting the measurements, the motors of sample and detector were adjusted by means of the goinometer to improve the optical path of the laser beam.

Vesicle spreading and protein binding was followed in situ in kinetic mode of the WASPLAS software (Fig. 3.27 B). Before and after each adlayer formation a spectrum was taken in scan mode $\left(\Theta_{i}=49-65^{\circ}\right.$ in buffered solution) (Fig. 3.27 A). A spectrum is characterized by a steep increase of reflectivity at TIR ( $\Theta_{c}=$ critical angle) followed by a sudden dip, at which the plasmon is excited (Fig. 3.27 A, black curve). An angle at the linear region $\left(\Theta_{\text {kin }}\right)$ right before the minimum is appropriate for kinetic measurements (Fig. $3.27 \mathrm{~B})$. There, the change of reflectivity $(\Delta R)$ is linearly depended on the thickness/refractive index change at the metal interface. Hence, membrane spreading or protein binding can be followed as a time course of the reflectivity shift using the kinetic mode. After the addition of the membrane/protein adlayer, another spectrum was recorded (Fig. 3.27 A, red curve), which is shifted to the right due to changing coupling conditions mentioned above. 

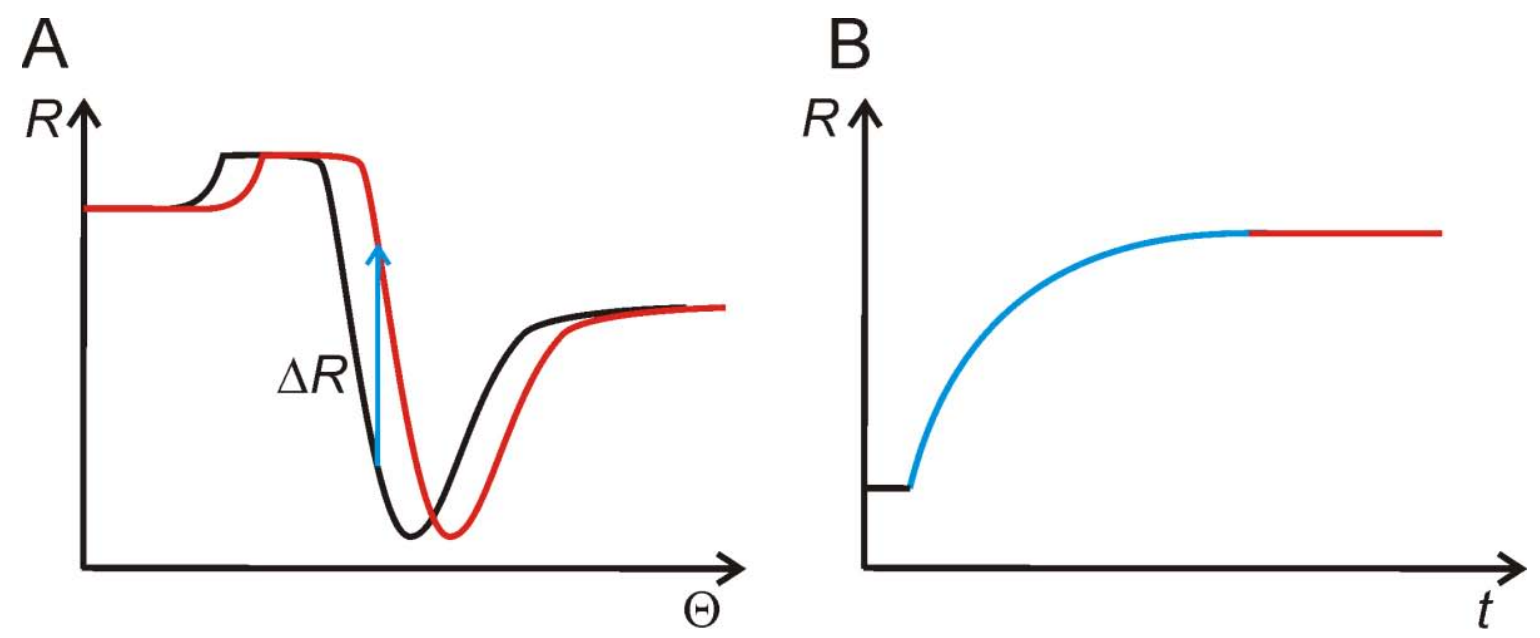

Fig. 3.27 (A) SPR spectrum taken before (black) and after adlayer formation (red) performed in scan mode ( $\Theta_{\mathrm{C}}$ is the critical angle at total internal reflection). (B) Time course of the reflectivity change at a certain angle $\left(\Theta_{\text {kin }}\right)$, the blue region shows the formation of an adlayer, the red baseline indicates saturation.

\section{Data evaluation}

SPR spectra were evaluated using the software WinSpall (freeware available online).$^{74}$ WinSpall software allows to manually fit the SPR spectra based on the Fresnel formalism by varying the thickness $d$ and reflective indices of each adlayer $\left(\varepsilon^{\prime}=\right.$ real part of dielectric constant and $\varepsilon^{\prime \prime}=$ imaginary part of dielectric constant). It is important only to fit the parameters of each adlayer at a time, because otherwise they would not be distinguishable. Knowing the approximate dielectric constants of each layer enables one to determine the thickness of each adsorbed material. All used dielectric constants are listed in Table 3.4. 
Table 3.4 Materials and corresponding dielectric constants $\left(\varepsilon^{\prime}=\right.$ real part, $\varepsilon^{\prime \prime}=$ imaginary part).

\begin{tabular}{c|c|c} 
Material & $\mathcal{E}^{\prime}$ & $\mathcal{E}^{\prime \prime}$ \\
\hline LaSFN9 glass & 3.404 & - \\
\hline chromium & -1.15 & 1.29 \\
\hline gold & $-(10.9-12.4)$ & - \\
\hline organics & 2.103 & - \\
\hline thiols, vesicles, proteins) & 1.778 & -
\end{tabular}

\subsubsection{Optical Waveguide Spectroscopy}

If the thickness of the optical transparent dielectric layer at the gold interface exceed a certain thickness (>500 nm) optical waveguide modes are excited instead or additionally to the surface plasmon by means of optical waveguide spectroscopy (OWS). ${ }^{75}$ In terms of their experimental setup both SPR and OWS are identical, but there are distinct differences described in the section below.

In this work, porous anodic aluminum oxide (AAO) films with a thickness of 3-4 $\mu \mathrm{m}$ and a pore diameter of 50-70 nm was utilized as transparent dielectric film. ${ }^{76}$ Adsorption processes that take place inside the porous area were followed in a time resolved manner i.e. vesicle spreading and protein binding within functionalized AAO pores. ${ }^{21,19}$ 


\section{Physical Background and Properties}

Laser light can be guided laterally inside an optical transparent AAO film, after being coupled inside the film via a semitransparent thin metal layer (i.e. gold). ${ }^{77}$ The light then zigzags through the medium (see Fig. $3.28 \mathrm{~A}$ ), if the condition of total internal reflection is fulfilled at both the AAO/gold and the AA0/outer environment respectively (see Snell's law, eq. 3.21 and Fig. 3.22 B. Hence, the refractive indices of both the gold $\left(n_{3}\right)$ and the environment (buffer, air, $n_{1}$ ) have to be smaller than the AAO refractive index $\left(n_{1}\right)$.

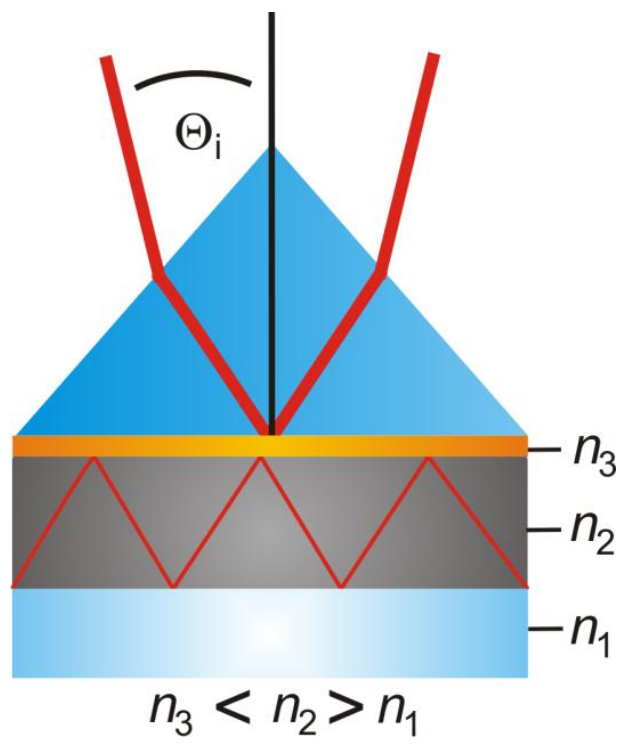

Fig. 3.28 Geometry of a planar slab waveguide.After coupling of the incoming light beam (red) with the angle of incidence $\Theta_{\mathrm{i}}$ The confined wave is guided in a high refractive index film $\left(n_{2}>n_{1}, n_{3}\right)$ with low attenuation.

In dependence on the film thickness and the effective dielectric properties, modes of different orders $\left(M_{\mathrm{i}}\right)$ can be detected at a respective coupling angle $\left(\Theta_{\mathrm{Mi}}\right)$. Modes are defined as the stationary properties of standing waves that can be compared with trajectories of a particle in a box with two adjacent low refractive index materials (gold, buffer) as barrier with large potential. Three of these modes and their appearance in a scan mode reflectivity curve are depicted in Fig. 3.29 A and B. The mode $M_{0}$ at $\Theta_{\mathrm{M} 0 \text { cor- }}$ 
responds to the surface plasmon that can only be detected, if the dielectric layer is sufficiently thin. One eminent advantage of OWS is that both types of linearly polarized light, p-polarized as well as s-polarized light (TE- and TM- polarized light) can be coupled into the dielectric film and multiple modes can be excided. This allows to determine more than one dielectric parameter for examples if the dielectric film has anisotropic dielectric constants i.e. porous AAO films $\left(\varepsilon_{\mathrm{x}}=\varepsilon_{\mathrm{y}}<\varepsilon_{\mathrm{z}}\right)$ evaluating the data collected in scan mode. Adsorption events in the pore interior alter the coupling conditions of the waveguide modes by enhancing the effective medium of the porous substrate leading to an increasing coupling angle (Fig. $3.29 \mathrm{C}$ ). The adsorption of particles (i.e. proteins, vesicles) inside the pores was followed using the angle tracking mode provided by the WASPLAS program.
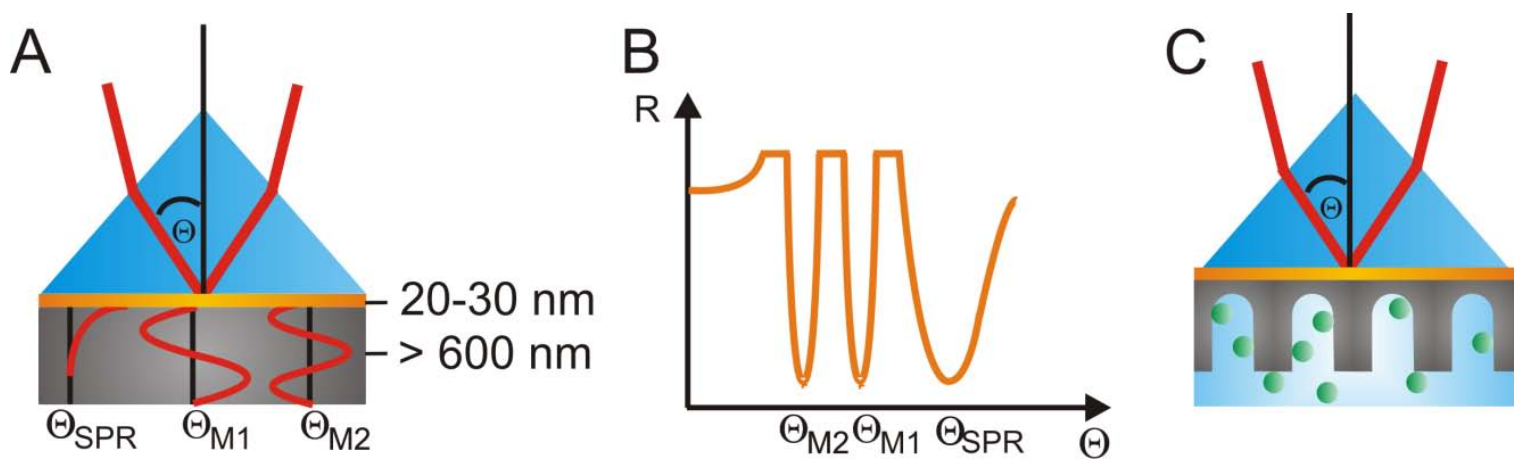

Fig. 3.29 (A) SPR/waveguide spectroscopy combination. At specific angles of incidents In addition to the SP $\left(\Theta_{\mathrm{sP}}\right)$ different waveguide modes $\left(\Theta_{\mathrm{M}}\right)$ can be excited. (B) Reflectivity $(\mathrm{R})$ as a function of angle of incident $(\Theta)$. Different dips of the spectra correspond to each waveguide mode. (C) Adsorption of particles (green circles) inside the AA0 pore interior.

\section{Effective medium theory based data evaluation}

The measured values of the dielectric constants can only be effective values ( $\left.\varepsilon_{\text {eff }}\right)$ as the AAO domain size $(d)$ is effectively smaller than the scattering limit of incident radiation ( $\sim 1 / 10 \lambda_{\text {light }}$. The contribution derives from both the anisotropic AAO dielectric constants $\varepsilon_{x, y}$ and $\varepsilon_{z}$ and the medium filling the pore interior ( $\left.\varepsilon_{\text {pore }}\right)$. To discriminate each 
individual dielectric constant, a Maxwell Garnett (MG) approach of the effective medium theory is adopted separately for the pore interior and AAO material assuming cylindrical shaped pores. ${ }^{78,7,79}$ The general approach is given by eq. 3.25:

$$
\varepsilon_{\text {eff }}=\varepsilon_{\text {matrix }} \cdot \frac{\varepsilon_{\text {matrix }}+\left(f_{\text {domain }}+f_{\text {matrix }} \cdot P\right) \cdot\left(\varepsilon_{\text {domain }}-\varepsilon_{\text {matrix }}\right)}{\varepsilon_{\text {matrix }}+f_{\text {matrix }} \cdot\left(\varepsilon_{\text {domain }}-\varepsilon_{\text {matrix }}\right) \cdot P},
$$

with $\varepsilon$ matrix as the dielectric constant of the continuous matrix of the porous support (here: $\left.\varepsilon_{\text {alumina }}\right), \varepsilon$ domain as the dielectric constant of pore interior ( $\left.\varepsilon_{\text {pore }}\right)$ and the fractions of $f_{\text {domain }}=1-f_{\text {matrix }}\left(f_{\text {pore }}=1-f_{\text {alumina }}\right)$. The depolarization factor $P$ is determined by the shape and orientation of the domains $(0 \leq P \geq 1)$. For cylindrical shaped pores the spatial contents of $P$ are given as $P_{\mathrm{x}}=P_{\mathrm{y}}=0.5$ and $P_{\mathrm{z}}=0$.

The pore diameter of the AAO porous substrate ( $\left.d_{\text {Pore }}\right)$ was first identified by comparing the constants of the alumina matrix $\varepsilon_{x, y}$ and $\varepsilon_{z}$ determined by OWS spectra fitting (TMand TE-mode) with the dielectric constants calculated by the MG approach (eq. 3.25, $\varepsilon_{x, y}=\varepsilon_{\text {eff }}$ with $P=0.5, \varepsilon_{x z}=\varepsilon_{\text {eff }}$ with $P=0$ ) using the geometric relation between the pore fraction $\left(f_{\text {pore }}\right)$ and pore diameter $d$ Pore given for hexagonal cylindrical pores. ${ }^{7}$

The dielectric response for the pore interior after particle deposition can be calculated by applying the MG equation. The pore interior now consists of an unfilled fraction $f_{\text {unfilled pore }}$ (buffer) and of the deposited material $f_{\text {material }}$ with the respective dielectric constants (eq. 3.26):

$\varepsilon_{\text {eff }}^{\text {pore }}=\varepsilon_{\text {unfilled pore }} \cdot \frac{\varepsilon_{\text {unfilled pore }}+\left(f_{\text {material }}+f_{\text {unfilled pore }} \cdot P\right) \cdot\left(\varepsilon_{\text {material }}-\varepsilon_{\text {unfilled pore }}\right)}{\varepsilon_{\text {unfilled pore }}+f_{\text {unfilled pore }} \cdot\left(\varepsilon_{\text {material }}-\varepsilon_{\text {unfilled pore }}\right) \cdot P}$,

with $\varepsilon_{\text {unfilled pore }}=\varepsilon$ buffer $=1.775$ and $\varepsilon_{\text {material }}=\varepsilon_{\text {organic }}=2.10$. 
The layer thickness of the particle adlayer can be then calculated from the material fraction by:

$$
d_{\text {organic }}=\frac{d_{\text {pore }}}{2} \cdot\left(1-\sqrt{1-f_{\text {material }}}\right)
$$

Thus, the thickness of each adlayer deriving from vesicle spreading or protein binding can be determined to ensure an appropriate membrane quality and protein binding.

\subsubsection{Confocal Laser Scanning Microscopy}

As a further development of standard epifluorescence techniques, the confocal laser scanning microscope (CLSM) offers the possibility to collect high resolution images of one focal plane and even compile three dimensional images or z-stacks. The CLSM technique has become invaluable for a wide range of medical, biological and biophysical investigations, imaging living and fixed cells, tissues or artificial membranes. ${ }^{80}$

In this work, the quality of solid supported hybrid membranes and bilayers as well as of pore spanning membranes (PSMs) that were doped with a fluorescent marker (Bodipy-PC or perylene, see Table 3.2, page 33) was controlled by means of the CLSM. Also, fluorescently labeled F-actin was visualized and investigated via the CLSM.

\section{Physical background}

The basic principle of a fluorescence microscope is the excitation of a fluorescently labeled specimen after illumination with light (source of light: xenon arc lamp, mercury vapor lamp). An electron of the molecule that serves as fluorescence label (see Table 3.2) will reach a higher electronic quantum state $\left(S_{1}\right)$ after being excited by light of a defined wavelength $\left(\lambda_{\mathrm{ex}}\right)$. It then relaxes back to the ground state $\left(\mathrm{S}_{0}\right)$ emitting a photon 
of higher wavelength $\left(\lambda_{\mathrm{em}}>\lambda_{\mathrm{ex}}\right)$. This so called Stokes shift is due to non-radiative vibronic relaxation of the electron in the higher quantum state. ${ }^{81}$

The confocal principle as the main difference of conventional epifluorescence microscope is presented in Fig. 3.30.

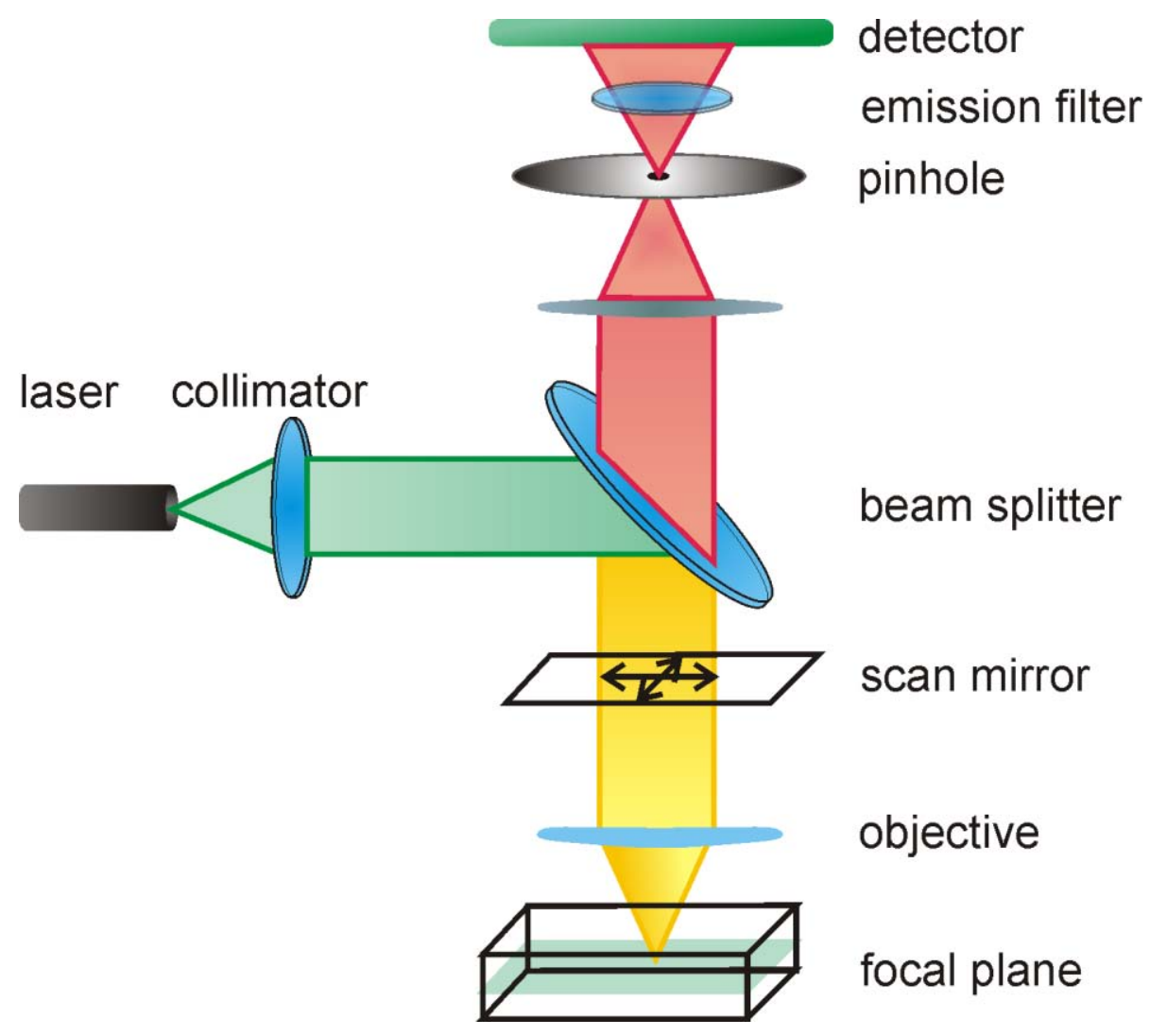

Fig. 3.30 Schematic setup and optical path of a confocal laser scanning microscope (CLSM).

Instead of incoherent lamp light, monochromatic laser light passes through a collimator to a beam splitter that separates the excitation from the emitted light in the beam path. The excitation light of the laser then hits a focal plane of the specimen after passing the objective lens. A scan mirror guides the focused laser beam pixel by pixel across the focal plane. A motorized z-control unit below the specimen allows to access any focal plane. The emitted light then passes through the beam splitter, a lens and an adjustable pinhole that excludes the out of focus light. A pinhole that is set to 1 airy unit (1 A.U.) 
further improves the $x-y$-resolution of an epifluorescence microscope by excluding higher orders of diffraction pattern. An emission filter ensures, that only light emitted with a certain wavelength reaches the detector (photomultiplier tube).

The $x-y$-resolution limit of an image $(d)$ is given by the law of Abbé (eq. 3.28):

$$
d=\frac{\lambda_{e x}}{2 n \cdot \sin \alpha}=\frac{\lambda_{e x}}{2 \cdot \mathrm{NA}}
$$

with $n$ being the refractive index of the lens medium and $\alpha$ the half angle of the objective. It can be enhanced using objective lenses with a high numerical aperture (NA). Mostly the $\mathrm{NA} \approx 1$, so that the $\mathrm{x}-\mathrm{y}$ resolution is approximately half of the laser wavelength. The z-resolution is significantly poorer with $\sim 1 \mu \mathrm{m}$.

In this work, the LSM 710 (Carl Zeiss, Jena, Germany) that was equipped with the software Zen 2008 and a water immersion $63 \times$ objective with NA = 1 (W Plan Achromat, Carl Zeiss, Jena, Germany) was used. To excite Bodipy-PC, Alexa Fluor488 Phalloidin and the Alexa Fluor488 C5-maleimide an Ar-ion laser $\left(25 \mathrm{~mW}, \lambda_{\mathrm{ex}}=488 \mathrm{~nm}\right.$, $\left.\lambda_{\mathrm{em}}=496-575 \mathrm{~nm}\right)$ was used, while for perylene excitation a diode laser $(30 \mathrm{~mW}$, $\left.\lambda_{\mathrm{ex}}=405 \mathrm{~nm}, \lambda_{\mathrm{em}}=409-479 \mathrm{~nm}\right)$ was chosen. To excite the TexasRed-maleimide a HeNelaser $\left(5 \mathrm{~mW}, \lambda_{\mathrm{ex}}=633 \mathrm{~nm}, \lambda_{\mathrm{em}}=638-648 \mathrm{~nm}\right)$ and for PIP 2 -TMR excitation a DPPS laser $\left(20 \mathrm{~mW}, \lambda_{\mathrm{ex}}=561 \mathrm{~nm}, \lambda_{\mathrm{em}}=574-619 \mathrm{~nm}\right)$ was used.

\section{Fluorescence recovery after photobleaching}

Fluorescence recovery after photobleaching (FRAP) is an important technique to study the lateral mobility of lipids in artificial membranes or living cells. ${ }^{82,83}$ Further methods to investigate lateral diffusion of lipids or proteins inside a membrane are fluorescence correlation spectroscopy (FCS) or single particle tracking (SPT).${ }^{84}$ 
Several theoretical models were developed in the past i.e. by Axelrod et al that enable one to determine the diffusion rate constant $D$ by fitting the fluorescnence recovery curve obtained during a FRAP experiment. ${ }^{85}$ A general FRAP curve as well as the corresponding fluorescence images are shown in Fig. $3.31 \mathrm{~A}$ and B. First of all, a round region of interest $(\mathrm{ROI})$ is defined. The ROI should be chosen in a way that it is located in the middle of a homogenous, defect free membrane patch. Then a high energetic laser pulse photochemically destroys the fluorescently marked molecules inside the ROI. After this short bleaching period ( 1-3 s) the fluorescence recovery inside the ROI due to lateral diffusion is recorded as a function of time (Fig. 3.31 A).

A

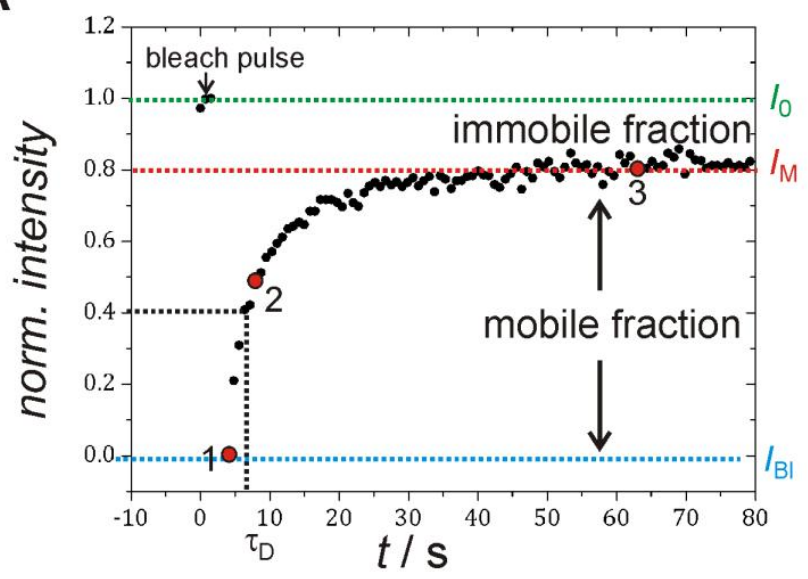

B
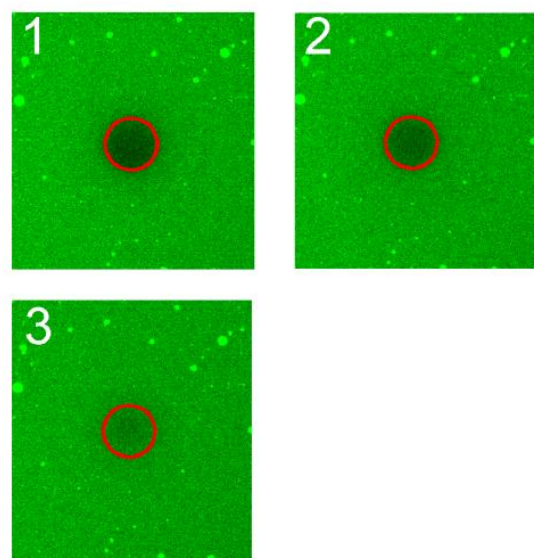

Fig. 3.31 (A) Normalized FRAP cuvre with essential parameters ( $I_{0}=$ normalized fluorescence intensity before bleaching, $I_{\mathrm{bl}}=$ intensity after bleaching, $I_{\mathrm{m}}=$ intesity after recovery). (B) Exemplary fluorescence images showing the time course of fluorescence recovery as observed after bleaching a ROI of a solid supported hydrbid membrane doped with Bodipy-PC. Numbers correspond to the moment marked in the FRAP curve.

Data evaluation a non normalized FRAP curve was performed using the built in software Zen2008 by fitting the recovery curve monoexponentially (eq. 3.29): 


$$
I(t)=I_{M}-\left(I_{M}-I_{B L}\right) \exp (-\tau \cdot t)
$$

with $I(\mathrm{t})=$ the fluorescence intensity at a certain time, $I_{\mathrm{M}}=$ the intensity after recovery, $I_{\mathrm{BL}}=$ the intensity after bleaching and $\tau=$ is the obtained fitting parameter.

From $\tau$ the half life of the recovery can be calculated (eq. 3.30):

$$
\tau_{\mathrm{D}}=\frac{\ln 0.5}{-\tau}
$$

The diffusion coefficient $D$ can be obtained by (eq. 3.31):

$$
D=\frac{r^{2}}{4 \cdot \tau_{\mathrm{D}}},
$$

with $r=$ radius or the ROI.

If the intensity after recovery $\left(I_{\mathrm{M}}\right)$ does not reach the level of the intensity before bleaching $\left(I_{0}\right)$ a cetrain immobile fraction is apparent. This fraction ( $\left.I_{\mathrm{IM}}\right)$ can be determined as follows: (eq. 3.32)

$$
I_{I M}=\frac{I_{0}-I_{M}}{I_{0}-I_{B l}}
$$


In this work, the quality of solid supported hybrid membranes and bilayers as well as pore spanning membranes was controlled by means of FRAP measurements. Diffusion coefficients and immobile fractions were extracted and compared to similar membrane systems.

\subsubsection{Scanning Electron Microscopy}

While the resolution $d$ of a light microscope is limited by the law of Abbé ( $d \sim 200 \mathrm{~nm}$ ) with a scanning electron microscope (SEM) it can be reduced to $1 \mathrm{~nm}$, since the wavelength of an electron beam is much lower compared to visible light. The main disadvantage is that only metal coated specimen that are stable in high vacuum can be visualized. Performing experiments in a liquid environment is thus impossible.

In this work, SEM was utilized to characterize and control anodic aluminum oxide (AAO) porous substrates, silicon nitride pores and cantilever. Prior to SEM imaging each specimen was coated with 5-30 nm gold by means of a Bal-Tec MCS610 thermal evaporation unit.

\section{Physical Background and Setup}

Instrumental setup and electron beam path are presented in Fig. 3.32. The electron gun generates electrons by thermionic emission from a tungsten filament at high temperatures under high vacuum ( $\left.\sim 5 \cdot 10^{-10} \mathrm{mbar}\right)$. The electrons are accelerated towards an anode $(0.2-30 \mathrm{kV})$. A condenser lens system then focuses the electron, while a magnetic field generated by scan coils deflects the electron beam back and forth. After being focused by the objective lens the specimen is scanned (electron beam spot size $~ 1-5 \mathrm{~nm}$ ) and backscattered or secondary electrons (SE) are detected by several possible detectors (here SE are detected by an in-lens or the SE2 defector and backscattered electrons by the BSE detector). ${ }^{86,87}$ 


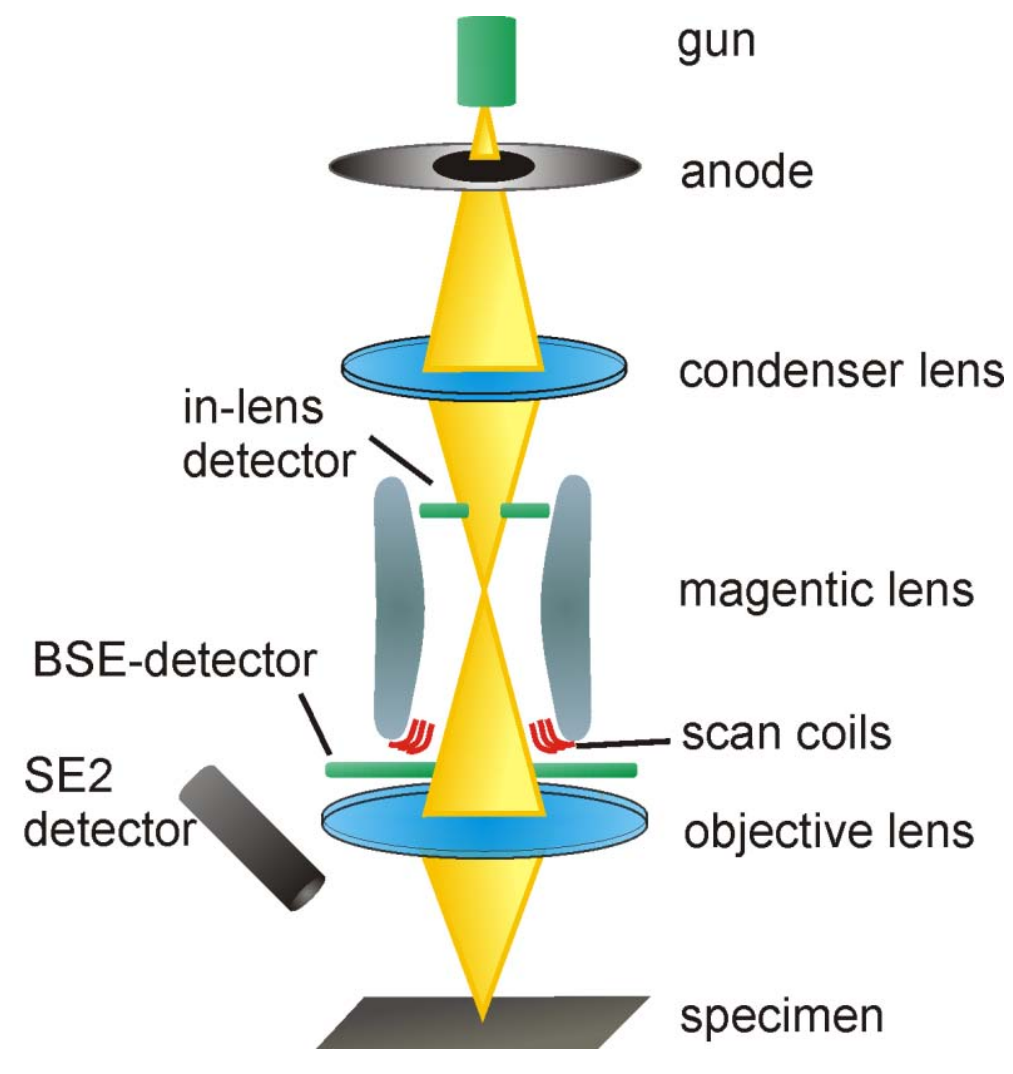

Fig. 3.32 Schematic daring of the scanning electron microscope (SEM) setup and optical path.

Interaction of highly accelerated electrons with the specimen surface produces a variety of electrons that are either reflected by or emitted from the substrate. Secondary electrons (SE) result from inelastic collision of the incident electrons with the specimen electrons. Loosely bound electrons of the specimens surface gain enough energy to be emitted. Hence, information about the specimen's topographic is provided. Backscattered electrons (BSE) are reflected electrons after elastic collision with the specimen. They penetrate deeper into the specimen compared to SE and thus provide information about the topographic and the atomic number contrast with a lower resolution than SE. Besides these electrons, X-rays and Auger electrons are generated, which give information about the specimen's composition. 


\section{Experimental Performance}

In this work, a LEO supra-35 SEM from Carl Zeiss (Jena, Germany) was used for SEM imaging. After flushing with nitrogen and obtaining a high vacuum $\left(5 \cdot 10^{-10} \mathrm{mbar}\right)$, electrons were accelerated with a gun power of $5 \mathrm{kV}$. Higher voltages normally lead to a higher resolution, if the material is highly conductive. However, higher voltages often cause charging effects or substrate damage. An in-lens SE detector that was perpendicular to the specimen surface was chosen to provide the best possible $x-y$ resolution (a $45^{\circ}$ SE2 detector in the contrary was used for z-topographic information). Contrast and brightness were automatically regulated and a standard aperture stop of $30 \mu \mathrm{m}$ was chosen. After focusing onto the specimen, the optical path of the electron beam was optimized by centering the aperture stop (aperture align/focus wobble) and correcting the astigmatism of the electron beam (stigmation $x, y$ ). This procedure had to be repeated in order to optimize the image quality.

\subsubsection{Atomic Force Microscopy}

Atomic force microscopy $(\mathrm{AFM})$ is a high resolution microscopic technique $(\mathrm{x}, \mathrm{y}-$ resolution $1 \mathrm{~nm}$, z-resolution $0.1 \mathrm{~nm}$ ) that was developed in the 1980's as a successor of the scanning tunneling microscope (STM). The AFM allows imaging the specimens surface by scanning it with a cantilever. By means of force spectroscopy the mechanical properties of the specimen can be characterized. Although it is a non-destructive technique that allows to examine biological specimen in an aqueous environment with a higher z-resolution than SEM, its drawbacks include long measuring times of only small scaled images ( $\mu \mathrm{m}$ range).

In this work, AFM was applied to investigate the mechanical properties of pore spanning membranes by means of indentation force maps and the influence of an attached F-actin network. Additionally it was utilized to image the porous structure of anodic aluminum oxide pores. 


\section{Physical Background and Setup}

The measuring principle of AFM is based on emerging forces between cantilever tip and surface of the specimen after the cantilever approaches the surface. A SEM micrograph of a cantilever is shown in Fig. 3.33 A together with an energy potential diagram (B) and force distance curve (C) that describes the behavior of a cantilever that comes in contact with a surface.
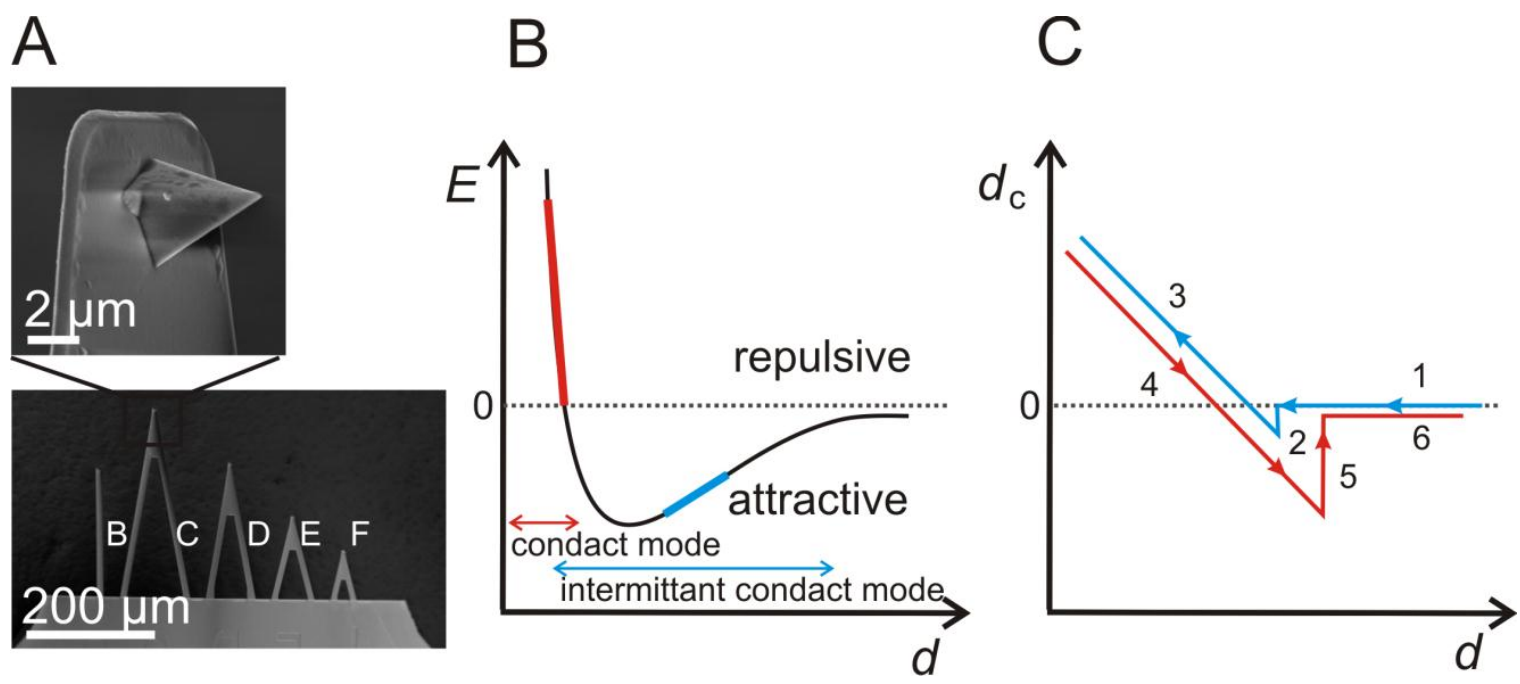

Fig. 3.33 (A) SEM micrograph of MLCT cantilever (B-F) (bottom) and the used C-cantilever (top) with a mean spring constant of $k=30 \mathrm{mN} / \mathrm{m}$. (B) Energy potential $(E)$ diagram and (C) idealized cantilever deflection $\left(d_{c}\right)$ versus distance $d$ curve: cantilever approaches the surfaces (1), snaps on (2) and gets deflected (3) the cantilever is retracted (4), loses contact (5) and is withdrawn from the surface.

A cantilever that scans the specimens topography gets bend due to attractive or repulsive forces from the surface (Fig. 3.34). This leads to deflection (lateral and/or vertical) of the laser beam that is reflected from the cantilever, which is detected by a photodiode. A feedback mechanism is employed that readjusts the cantilever displacement via a z-piezo element. Piezo crystals of the $z$ and the $x, y$ element respectively contract or expand in the presence of a voltage, so that three dimensional movements are possible. The cantilever deflection $(d c)$ is linearly proportional to the force $(F)$ that acts on the tip (Hooke's law, eq. 3.33): 


$$
F=k_{\mathrm{C}} \cdot d_{\mathrm{C}}
$$

Hence, with a priory determined cantilever spring concstant $\left(k_{c}\right)$ the force can be calculated.

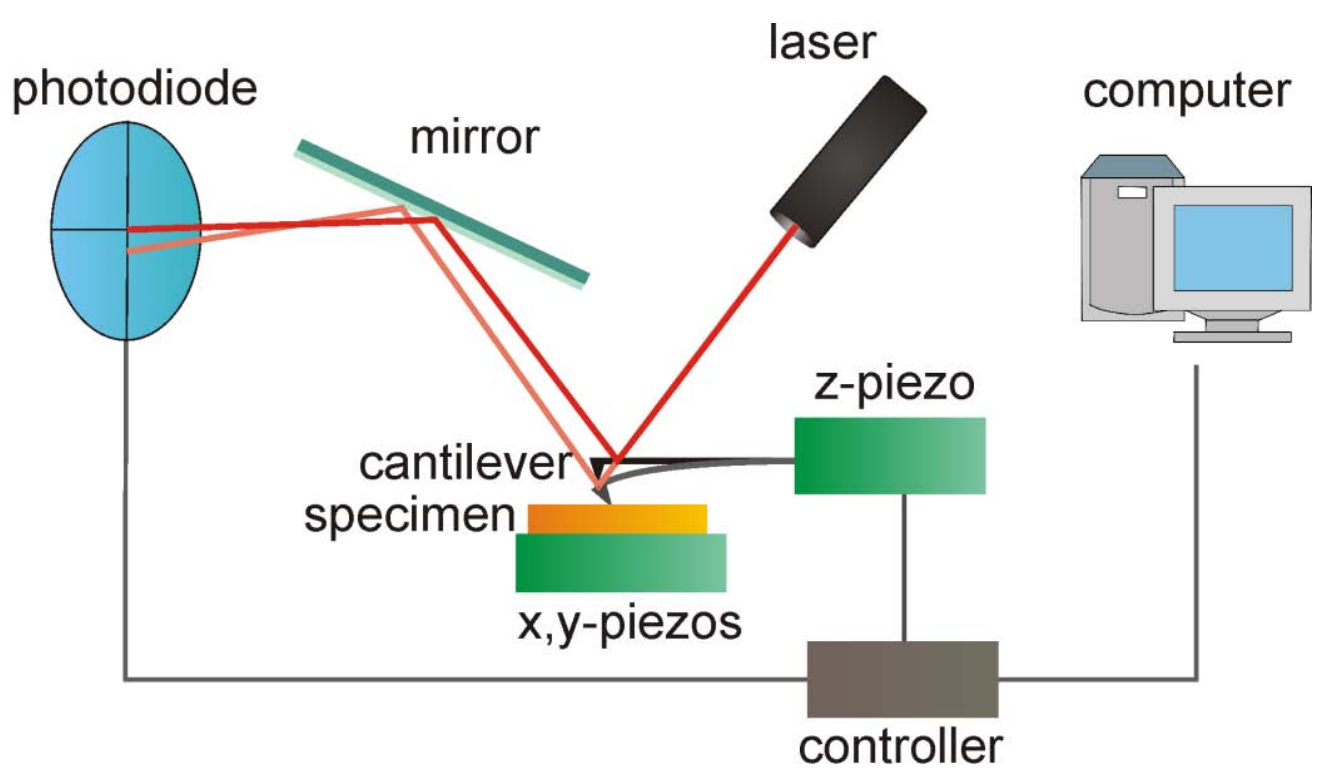

Fig. 3.34 Schematic drawing of an AFM setup.

AFM imaging can either be performed in contact or intermittent contact mode (so called tapping mode). In contact mode, the generally scans the surface with a constant force, where the deflection setpoint is kept constant. In intermittent contact mode, the cantilever constantly oscillates close to its resonance frequency with a constant amplitude that its readjusted by a feedback loop.

Beside the imaging mode, force distance curves can be employed to measure mechanical properties of cells or artificial membranes as well as interaction forces between biomolecules. ${ }^{88,29,89}$ By means of indentation experiments performed on cells or artificial 
membranes, the stiffness (Young's modulus, elastic response) and viscoelastic response can be examined. Also, the contribution of cellular components i.e. the cytoskeleton to the mechanical properties can be determined. Therefore, force distance curves are recorded and plotted as the force response $F$ of the cantilever against the sample-tip distance $d$ (see Fig. 3.33 C). As the cantilever gets in contact to the surface (Fig. 3.33 C, blue curve, section 3), repulsion from the surface leads to a cantilever deflection. The force response can be calculated from the cantilever deflection, if the spring constant of the cantilever is known. The slope of the corresponding curve is, in case of pore spanning membranes, mainly determined by the lateral tension of the membrane, so that the apparent spring constant $k_{\text {app }}$ can be evaluated by linear regression.

A non-elastic behavior of the specimen during indentation/retraction reflected in a hysteresis between the indentation and retraction curve. This phenomenon is an indication of energy loss during the indentation/retraction process, caused by viscoelastic effects. The cantilever response during retraction gives further information about adhesion or stretching phenomena.

\section{Experimental Performance}

Force mapping indentation experiments of pore spanning membranes with and without an attached F-actin network were performed with the MFP-3D stand alone AFM from Asylum Research (Santa Barbara, USA) using a soft, triangular MLCT lever $(k c=10-60 \mathrm{mN} / \mathrm{m}$, see Fig. 3.33 A) from AFM Probes (Camarillo, USA). Force maps consisting of $40 \times 40$ force distance curves (images size $7 \times 7 \mu \mathrm{m}^{2}$ ) were performed with different approaching velocities $(v=0.3-20 \mu \mathrm{m} / \mathrm{s})$.

Prior to each experiment, reference curves on a hard surface (silicon nitride) were recorded to determine both, the contact point of the cantilever tip and the sensitivity of the photodiode (deflection signal/ output voltage) by linear regression of the obtained curve. 
Before force distance curves were recorded, the exact spring constant of the cantilever $\left(k_{c}\right)$ was determined using the thermal noise calibration method (see eq. 3.33). The spring constant is obtained from eq. 3.34:

$$
k_{C}=\frac{k_{\mathrm{B}} \cdot T}{\left\langle z^{2}\right\rangle}
$$

with the mean square deflection $\left\langle z^{2}\right\rangle$ of the cantilever arousing from thermal fluctuations. The method is based on the assumption that the mean thermal energy of the system is $0.5 k_{\mathrm{B}} \mathrm{T}$ per degree of freedom (equipartition theorem). 


\subsection{References}

[1] Thompson, G. (1997) Porous anodic alumina: fabrication, characterization and applications. Thin Solid Films 297, 192-201.

[2] Steltenkamp, S., Müller, M. M., Deserno, M., Hennesthal, C., Steinem, C., and Janshoff, A. (2006) Mechanical properties of pore-spanning lipid bilayers probed by atomic force microscopy. Biophys. J. 91, 217-226.

[3] Drexler, J., and Steinem, C. (2003) Pore-Suspending Lipid Bilayers on Porous Alumina Investigated by Electrical Impedance Spectroscopy. J. Phys. Chem. B $107,11245-11254$.

[4] Li, F., Zhang, L., and Metzger, R. M. (1998) On the Growth of Highly Ordered Pores in Anodized Aluminum Oxide. Chem. Mater 10, 2470-2480.

[5] Jessensky, O., Müller, F., and Gösele, U. (1998) Self-organized formation of hexagonal pore arrays in anodic alumina. Appl. Phys. Lett 72, 1173.

[6] Li, A. P., Müller, F., Birner, A., Nielsch, K., and Gösele, U. (1998) Hexagonal pore arrays with a 50-420 nm interpore distance formed by self-organization in anodic alumina. J. Appl. Phys. 84, 6023.

[7] Lazzara, T. D. (2011) Directing macromolecular assemblies by tailored surface functionalizations of nanoporous alimuna. Dissertation, Göttingen.

[8] O'Sullivan, J. P., and Wood, G. C. (1970) The Morphology and Mechanism of Formation of Porous Anodic Films on Aluminium. P. Roy. Soc. A-Mat. Phy. 317, 511-543.

[9] Masuda, H., and Fukuda, K. (1995) Ordered metal nanohole arrays made by a two-step replication of honeycomb structures of anodic alumina. Science 268, $1466-1468$. 
[10] Lo, D., and Budiman, R. A. (2007) Fabrication and Characterization of Porous Anodic Alumina Films from Impure Aluminum Foils. J. Electrochem. Soc. 154, C60.

[11] Oh, H. J., Jang, K. W., Lee, J. H., Ki, B. S., Heo, C. H., Kwon, S. H., and Chi, C. S. (2006) Effects of Annealing Treatment of Aluminum Substrate on Nanopore Arrangements in Anodic Alumina. Sol. St. Phen. 118, 549-554.

[12] Haensch, C., Hoeppener, S., and Schubert, U. S. (2010) Chemical modification of self-assembled silane based monolayers by surface reactions. Chem Soc Rev 39, 2323-2334.

[13] Poirier, and Pylant (1996) The Self-Assembly Mechanism of Alkanethiols on $\mathrm{Au}(111)$. Science 272, 1145-1148.

[14] Bain, C. D., Troughton, E. B., Tao, Y. T., Evall, J., Whitesides, G. M., and Nuzzo, R. G. (1989) Formation of monolayer films by the spontaneous assembly of organic thiols from solution onto gold. J. Am. Chem. Soc. 111, 321-335.

[15] Wasserman, S. R., Tao, Y. T., and Whitesides, G. M. (1989) Structure and reactivity of alkylsiloxane monolayers formed by reaction of alkyltrichlorosilanes on silicon substrates. Langmuir 5, 1074-1087.

[16] Love, J. C., Estroff, L. A., Kriebel, J. K., Nuzzo, R. G., and Whitesides, G. M. (2005) Self-assembled monolayers of thiolates on metals as a form of nanotechnology. Chem. Rev 105, 1103-1169.

[17] Carson, G., and Granick, S. (1989) Self-assembly of octadecyltrichlorosilane films on mica. J. Appl. Polym. Sci. 37, 2767-2772.

[18] Brzoska, J. B., Azouz, I. B., and Rondelez, F. (1994) Silanization of Solid Substrates: A Step Toward Reproducibility. Langmuir 10, 4367-4373.

[19] Lazzara, T. D., Kliesch, T.-T., Janshoff, A., and Steinem, C. (2011) Orthogonal functionalization of nanoporous substrates: control of 3D surface functionality. ACS Appl Mater Interfaces 3, 1068-1076. 
[20] Bezanilla, M., Manne, S., Laney, D. E., Lyubchenko, Y. L., and Hansma, H. G. (1995) Adsorption of DNA to Mica, Silylated Mica, and Minerals: Characterization by Atomic Force Microscopy. Langmuir 11, 655-659.

[21] Lazzara, T. D., Behn, D., Kliesch, T.-T., Janshoff, A., and Steinem, C. (2012) Phospholipids as an alternative to direct covalent coupling: surface functionalization of nanoporous alumina for protein recognition and purification. J. Colloid. Interf. Sci. 366, 57-63.

[22] Kocun, M., Lazzara, T. D., Steinem, C., and Janshoff, A. (2011) Preparation of solvent-free, pore-spanning lipid bilayers: modeling the low tension of plasma membranes. Langmuir 27, 7672-7680.

[23] Mathivet, L., Cribier, S., and Devaux, P. F. (1996) Shape change and physical properties of giant phospholipid vesicles prepared in the presence of an AC electric field. Biophys. J. 70, 1112-1121.

[24] Lingler, S., Rubinstein, I., Knoll, W., and Offenhäusser, A. (1997) Fusion of Small Unilamellar Lipid Vesicles to Alkanethiol and Thiolipid Self-Assembled Monolayers on Gold. Langmuir 13, 7085-7091.

[25] Plant, A. L. (1999) Supported hybrid bilayer membranes as rugged cell membrane mimics. Langmuir 15, 5128-5135.

[26] Castellana, E. T., and Cremer, P. S. (2007) Solid-Supported Lipid Bilayers: From Biophysical Studies to Sensor Design. ChemInform 38.

[27] Richter, R. P., Bérat, R., and Brisson, A. R. (2006) Formation of solid-supported lipid bilayers: an integrated view. Langmuir 22, 3497-3505.

[28] Mey, I., Steinem, C., and Janshoff, A. (2012) Biomimetic functionalization of porous substrates: towards model systems for cellular membranes. J. Mater. Chem.

[29] Mey, I., Stephan, M., Schmitt, E. K., Müller, M. M., Ben Amar, M., Steinem, C., and Janshoff, A. (2009) Local membrane mechanics of pore-spanning bilayers. J. Am. Chem. Soc. 131, 7031-7039. 
[30] Baneyx, F. (1999) Recombinant protein expression in Escherichia coli. Curr. Opin. Biotechnol. 10, 411-421.

[31] Sørensen, H. P., and Mortensen, K. K. (2005) Soluble expression of recombinant proteins in the cytoplasm of Escherichia coli. Microb. Cell Fact. 4, 1.

[32] Boesler, B., Müller, S., Überexpression rekombinanter Proteine. BiuZ 38, 287.

[33] Expasy, http://web.expasy.org/protparam/ (2012), cited on 2012 Aug. 29.

[34] Qiagen FAQ No. 132, http://www.qiagen.com/faq/ (2012) cited on 2012 Dec. $9 . \quad$.

[35] Olson, B. J., and Markwell, J. Assays for Determination of Protein Concentration.

[36] Hoffmeister, H. (2008) Struktur- und Funktionsanalyse von Polycystin-2. Disser tation, Regensburg.

[37] Bradford, M. M. (1976) A rapid and sensitive method for the quantitation of microgram quantities of protein utilizing the principle of protein-dye binding. Anal. Biochem. 72, 248-254.

[38] Zor, T., and Selinger, Z. (1996) Linearization of the Bradford protein assay in creases its sensitivity: theoretical and experimental studies. Anal. Biochem 236, 302-308.

[39] Bosk, S., Braunger, J. A., Gerke, V., and Steinem, C. (2011) Activation of F-actin binding capacity of ezrin: synergism of $\mathrm{PIP}_{2}$ interaction and phosphorylation. Bio phys. J. 100, 1708-1717.

[40] Invitrogen, http://products.invitrogen.com/ivgn/product/A10254 (2012) cited on 2012 Dec. 9.

[41] Cytoskeleton, datasheets. http://www.cytoskeleton.com/actin/proteins (2012) cited on 2012 Dec. 9.

[42] Cooper, J. A. (1987) Effects of cytochalasin and phalloidin on actin. J. Cell. Biol. 105, 1473-1478. 
[43] Tarantola, M., Sunnick, E., Schneider, D., Marel, A.-K., Kunze, A., and Janshoff, A. (2011) Dynamic changes of acoustic load and complex impedance as reporters for the cytotoxicity of small molecule inhibitors. Chem. Res. Toxicol 24, 1494-1506.

[44] Steinem, C., Janshoff, A., Ulrich, W. P., Sieber, M., and Galla, H. J. (1996) Impedance analysis of supported lipid bilayer membranes: a scrutiny of different prepa-ration techniques. Biochim. Biophys. Acta 1279, 169-180.

[45] Steinem, C., Janshoff, A., Galla, H.-J., and Sieber, M. (1997) Impedance analysis of ion transport through gramicidin channels incorporated in solid supported lipid bilayers. Bioelectroch. Bioener. 42, 213-220.

[46] Kelly, S. M., Jess, T. J., and Price, N. C. (2005) How to study proteins by circular dichroism. BBA-Proteins Proteom. 1751, 119-139.

[47] Ranjbar, B., and Gill, P. (2009) Circular dichroism techniques: biomolecular and nanostructural analyses- a review. Chem. Biol. Drug Des. 74, 101-120.

[48] DICHROWEB, http://dichroweb.cryst.bbk.ac.uk (2012), cited on 2012 Dec. 9.

[49] SDS Gel Electrophoresis, http://mach7.bluehill.com/proteinc/tutorial/ sdspage.html (2012) cited on 2012 Dec. 9.

[50] Kelly, S., and Price, N. (2000) The Use of Circular Dichroism in the Investigation of Protein Structure and Function. curr protein pept sci 1, 349-384.

[51] Sauerbrey, G. (1959) Verwendung von Schwingquarzen zur Wägung dünner Schichten und zur Mikrowägung. Z. Phys. 155, 206-222.

[52] Janshoff, Galla, and Steinem (2000) Piezoelectric Mass-Sensing Devices as Biosensors-An Alternative to Optical Biosensors? Angew. Chem. Int. Ed. Engl 39, 4004-4032.

[53] Minunni, M., Mascini, M., Guilbault*, G. G., and Hock, B. (1995) The Quartz Crystal Microbalance as Biosensor. A Status Report on Its Future. Analytical Letters 28, 749-764. 
[54] Cheng, C. I., Chang, Y.-P., and Chu, Y.-H. (2012) Biomolecular interactions and tools for their recognition: focus on the quartz crystal microbalance and its diverse surface chemistries and applications. Chem Soc Rev 41, 1947-1971.

[55] Becker, B., and Cooper, M. A. (2011) A survey of the 2006-2009 quartz crystal microbalance biosensor literature. J. Mol. Recognit. 24, 754-787.

[56] Caruso, F., Rodda, E., Furlong, D. N., Niikura, K., and Okahata, Y. (1997) Quartz crystal microbalance study of DNA immobilization and hybridization for nucleic acid sensor development. Anal. Chem 69, 2043-2049.

[57] Herrig, A., Janke, M., Austermann, J., Gerke, V., Janshoff, A., and Steinem, C. (2006) Cooperative Adsorption of Ezrin on PIP 2 -Containing Membranes. Biochemistry 45, 13025-13034.

[58] Kastl, K., Ross, M., Gerke, V., and Steinem, C. (2002) Kinetics and thermodynamics of annexin A1 binding to solid-supported membranes: a QCM study. Biochemistry 41, 10087-10094.

[59] Janshoff, A., Steinem, C., Sieber, M., and Galla, H.-J. (1996) Specific binding of peanut agglutinin to G M1 -doped solid supported lipid bilayers investigated by shear wave resonator measurements. Eur. Biophys. J. 25, 105-113.

[60] Wegener, J., Janshoff, A., and Steinem, C. (2001) The quartz crystal microbalance as a novel means to study cell-substrate interactions in situ. Cell Biochem. Biophys. 34, 121-151.

[61] Behn, D., Bosk, S., Hoffmeister, H., Janshoff, A., Witzgall, R., and Steinem, C. (2010) Quantifying the interaction of the C-terminal regions of polycystin-2 and polycystin-1 attached to a lipid bilayer by means of QCM. Biophys. Chem. 150, $47-53$.

[62] Kaspar, M., Stadler, H., Weiss, T., and Ziegler, C. (2000) Thickness shear mode resonators ("mass-sensitive devices") in bioanalysis. Fresen. J. Anal. Chem. 366, 602-610. 
[63] O'Sullivan, C., and Guilbault, G. (1999) Commercial quartz crystal microbalances - theory and applications. Biosens. Bioelectron. 14, 663-670.

[64] Rickert, J., Brecht, A., and Göpel, W. (1997) QCM Operation in Liquids: Constant Sensitivity during Formation of Extended Protein Multilayers by Affinity. Anal. Chem 69, 1441-1448.

[65] Thompson, M., Kipling, A. L., Duncan-Hewitt, W. C., Rajakovi, L. V., and ČavićVlasak, B. A. (1991) Thickness-shear-mode acoustic wave sensors in the liquid phase. A review. Analyst 116, 881.

[66] Reiss, H., Frisch, H. L., and Lebowitz, J. L. (1959) Statistical Mechanics of Rigid Spheres. J. Chem. Phys. 31, 369.

[67] Lüthgens, E. (2005) Modellierung der Oberflächenreaktionen von Vesikeln und Proteinen mit Hilfe dynamischer Monte Carlo - Simulationen. Dissertation, Mainz.

[68] Kastl, K., Menke, M., Lüthgens, E., Faiß, S., Gerke, V., Janshoff, A., and Steinem, C. (2006) Partially Reversible Adsorption of Annexin A1 on POPC/POPS Bilayers In-vestigated by QCM Measurements, SFM, and DMC Simulations. ChemBio Chem. 7, 106-115.

[69] Fan, X., White, I. M., Shopova, S. I., Zhu, H., Suter, J. D., and Sun, Y. (2008) Sensitive optical biosensors for unlabeled targets: A review. Analytica Chimica Acta $620,8-26$.

[70] Ramsden, J. J. (1997) Optical biosensors. J. Mol. Recognit. 10, 109-120.

[71] Baird, C. L., and Myszka, D. G. (2001) Current and emerging commercial optical biosensors. J. Mol. Recognit. 14, 261-268.

[72] Zayats, A. V., Smolyaninov, I. I., and Maradudin, A. A. (2005) Nano-optics of surface plasmon polaritons. Phys. Rep. 408, 131-314.

[73] Res-Tec, http://www.res-tec.de/. (2012) cited on 2012 Dec. 9. 
[74] WINSPALL Software download, http://www.mpip-mainz.mpg.de/groups/knoll/ software, (2012) cited on 2012 Dec. 9.

[75] Knoll, W. (1998) Interfaces and thin films as seen by bound electromagnetic waves. Annu Rev Phys Chem 49, 569-638.

[76] Yamaguchi, A., Hotta, K., and Teramae, N. (2009) Optical Waveguide Sensor Based on a Porous Anodic Alumina/Aluminum Multilayer Film. Anal. Chem. 81, 105-111.

[77] Knoll, W. (1998) Interfaces and thin films as seen by bound electromagnetic waves. Annu Rev Phys Chem 49, 569-638.

[78] Lazzara, T. D., Lau, K. H. A., and Knoll, W. (2010) Mounted nanoporous anodic alumina thin films as planar optical waveguides. J. Nanosci. Nanotechno. 10, 42934299.

[79] Foss, C. A., Tierney, M. J., and Martin, C. R. (1992) Template synthesis of infrared transparent metal microcylinders: comparison of optical properties with the predictions of effective medium theory. J. Phys. Chem. 96, 9001-9007.

[80] Paddock, S. W. (1999) Confocal laser scanning microscopy. Biotechniques 27, 9926, 998-1002, 1004.

[81] Lichtman, J. W., and Conchello, J.-A. (2005) Fluorescence microscopy. Nat Meth 2, 910-919.

[82] Jacobson, K., Derzko, Z., Wu, E.-S., Hou, Y., and Poste, G. (1976) Measurement of the lateral mobility of cell surface components in single living cells by fluorescence recovery after photobleaching. J. Supramol. Str. Cell 5, 565-576.

[83] Ladha, S., Mackie, A., Harvey, L., Clark, D., Lea, E., Brullemans, M., and Duclohier, H. (1996) Lateral diffusion in planar lipid bilayers: a fluorescence recovery after photobleaching investigation of its modulation by lipid composition, cholesterol, or alamethicin content and divalent cations. Biophys. J. 71, 1364-1373. 
[84] Chen, Y., Lagerholm, B. C., Yang, B., and Jacobson, K. (2006) Methods to measure the lateral diffusion of membrane lipids and proteins. Methods 39, 147-153.

[85] Axelrod, D., Koppel, D. E., Schlessinger, J., Elson, E., and Webb, W. W. (1976) Mobility measurement by analysis of fluorescence photobleaching recovery kinetics. Biophys. J. 16, 1055-1069.

[86] SubsTech, http://www.substech.com/dokuwiki/doku.php?id=scanning _electron_micros-cope\&s=sem (2012) cited on 2012 Dec. 9.

[87] Cheney, B., Sem Guide, http://www.sjsu.edu/people/anastasia.micheals/courses/MatE143/s1/SEM_GUIDE.pdf (2012) cited on 2012 Dec. 9

[88] Lorenz, B., Mey, I., Steltenkamp, S., Fine, T., Rommel, C., Müller, M. M., Maiwald, A., Wegener, J., Steinem, C., and Janshoff, A. (2009) Elasticity Mapping of Pore-Suspending Native Cell Membranes. Small 5, 832-838.

[89] Lorenz, B., Álvarez Cienfuegos, L. de, Oelkers, M., Kriemen, E., Brand, C., Stephan, M., Sunnick, E., Yüksel, D., Kalsani, V., Kumar, K., Werz, D. B., and Jan shoff, A. (2012) Model system for cell adhesion mediated by weak carbohydratecarbohydrate interactions. J. Am. Chem. Soc. 134, 3326-3329. 


\section{Biosensor Assay Development Based on Solid Supported Membranes: Studying Protein-Protein Interactions}

\subsection{Abstract}

The transmembrane protein polycystin-2 (PC2) is a member of the transient receptor potential (TRP) family and functions as a $\mathrm{Ca}^{2+}$-permeable, non selective cation channel. PC2 is in the centre of a protein interaction network, where interactions are mediated by its $\mathrm{C}$-terminus (cPC2). These interactions are claimed to be responsible for regulating cellular proliferation and differentiation.

Here, developed a membrane based biosensor assay that mimics the natural environment of the membrane anchored protein fragments. This platform can serve as a screening assay, that would allow us to determine the binding affinities and kinetic rate constants of $\mathrm{cPC} 2$ and its interaction partners in order to evaluate their biological relevance.

As an accepted cPC2 interaction partner, $C$-terminal polycystin-1 (cPC1) was employed to build up the a membrane based biosensor assay using two independent techniques, the surface plasmon resonance (SPR) and quartz crystal microbalance (QCM). Specific immobilization of the hexahistidine tagged protein $\mathrm{CPC} 1$ on a solid supported DOPC membrane doped with 10 mol\% DOGS-NTA-Ni and subsequent cPC2 binding and desorption was monitored by means of the two biosensor techniques. Binding affinities 
and kinetic rate constants were evaluated and compared in order to elucidate the applicability of the SPR and QCM instruments as biosensor devices.

\subsection{Introduction}

As a member of the transient receptor potential (TRP) channel family, Polycystin-2 (PC2) or TRPP2 functions as a $\mathrm{Ca}^{2+}$ permeable, non-selective cation channel that plays a fundamental role in cell development and proliferation. The gene $p k d 2$ that encodes for PC2 is one of at least two genes, whose mutation leads to the development of renal cysts and eventually causes renal failure. This autosomal polycystic kidney disease (ADPKD) is one of the most common monogenetic disorders with a prevalence of 1:1000. PC2 is composed of 968 amino acids ( 110 kDa) with six membrane spanning regions and intracellular $N$ - and $C$-terminal domains. The $C$-terminus of PC2 (cPC2), especially its coiled-coil domain, has been identified as a region with many potential interaction partners (see Fig. 4.1). 


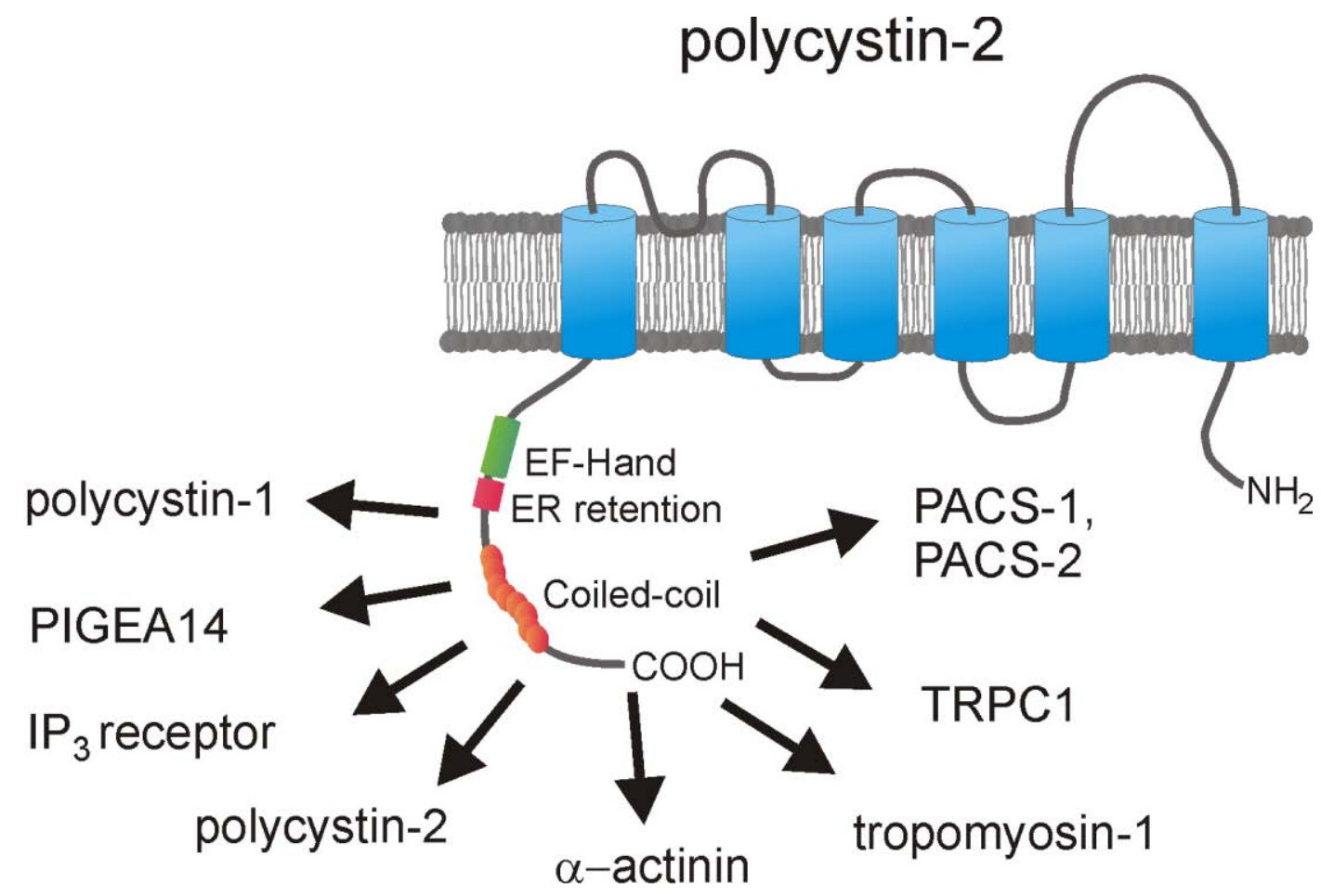

Fig. 4.1 Schematic drawing of polycystin-2 embedded in a cellular membrane. A selection of potential interaction partners, that putatively interacts with the C-terminal region of polycystin-2 are listed.

The topology of the C-terminal PC2 (Ile $\left.e^{680}-\mathrm{Val}^{968}\right)$ is depicted in Fig. $4.2 \mathrm{~A}$ together with the structural model (Fig. 4.2 B) obtained from ROBETTA by Célic et al. in 2008. ${ }^{1}$ As structural motifs, it harbors an EF hand domain as a potential $\mathrm{Ca}^{2+}$ binding site, a retention signal to the endoplasmatic reticulum (ER) and most interestingly one accepted and one proposed coiled-coil domain, which have been narrowed down as interaction sites of some of its potential interaction partners. 


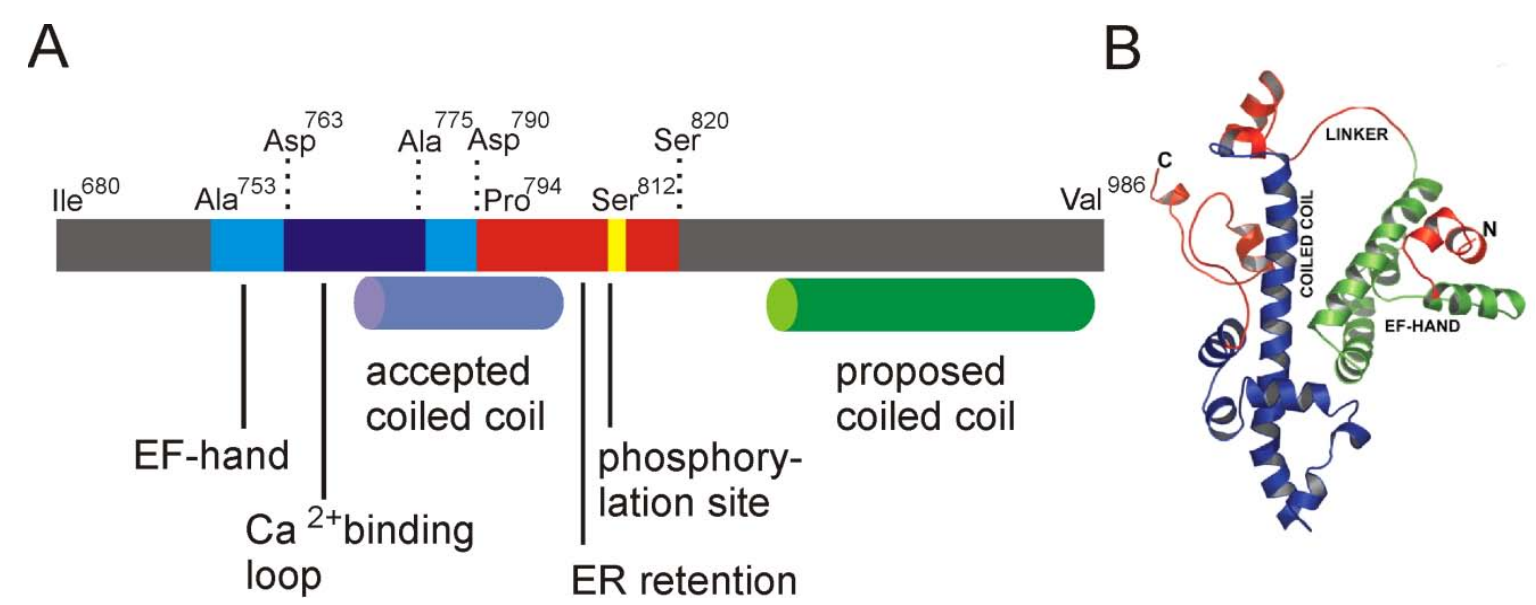

Fig. 4.2 (A) Topology of the cPC2 predicted domains (Ile $\left.{ }^{680}-\mathrm{Va}{ }^{968}\right)$. (B) Model of cPC2 (Ile $\left.{ }^{704-V a l}{ }^{968}\right)$ obtained from ROBETTA. ${ }^{1}$

The interactions between CPC2 and these proteins however, have yet not been investigated in a quantitative manner. However, quantifying the interactions would be of great interest, because it offers the possibility to distinguish those that are of biological relevance, indicated by a strong affinity, from those, which can biologically be neglected.

One of this interaction partners is a proteins named polycystin-1 (PC1). PC1 ( 4003 amino acids, $462 \mathrm{kDa}$ ) is encoded by $p k d 1$, the second gene, whose mutation causes ADPKD. This plasma membrane anchored protein is composed of 11 transmembrane spanning regions with an extracellular $N$-terminus and an intracellular $C$ terminal region. ${ }^{2}$ By means of a co-immunuprecipitation assay Hanaoka et al. found that a coiled-coil domain within the $C$-terminal domain of PC1 was sufficient to mediate cPC1-cPC2 complex formation. ${ }^{3}$

Due to its large extracellular N-terminus, PC1 is capable of sensing fluid shear stress, being localized in the ciliary plasma membrane. This stress causes a conformational changes, which facilitates the interaction of the two intracellular coiled-cloil domains of PC1 and PC2. PC2 in turn produces a $\mathrm{Ca}^{2+}$ influx, leading to a total increase of intracellular $\mathrm{Ca}^{2+}$ concentration and thus to cellular activities, which regulate cell development and growth. ${ }^{4}$ A schematic drawing of the PC2/PC1 complex is shown in Fig. 4.3. 


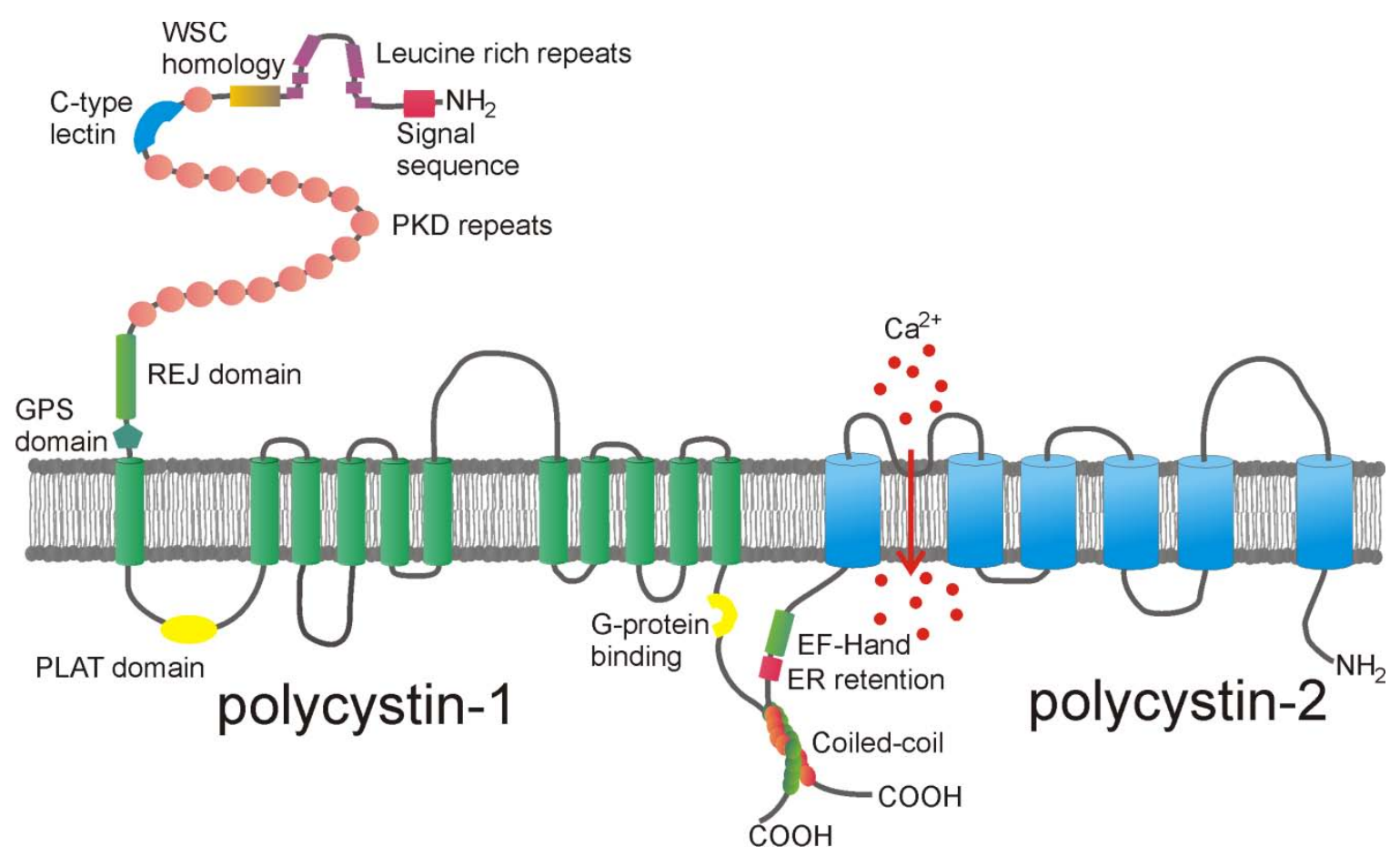

Fig. 4.3 Schematic drawing of the domain structure of polycystin-1 and polycystin-2 during complex formation.

The sequence of cPC1 used in this work (136 amino acids, original PC1 sequence from Ser $\left.{ }^{4192-T h r}{ }^{4303}\right)$ is depicted in Fig. 4.4 A together with a helical wheel diagram of the coiled coil domain (Arg $\left.{ }^{4214}-G l y{ }^{4248}\right)$ in Fig. 4.4 B. 
A

$\begin{array}{cccccr}10 & 20 & 30 & 40 & 50 & 60 \\ \text { MASMTGGQQM } & \text { GRDPSQLDGL SVSLGRLGTR CEPEPSRLQA VFEALLTQFD RLNQATEDVY } \\ 70 & 80 & 90 & 100 & 110 & 120 \\ \text { QLEQQLHSLQ } & \text { GRSSSRAPAG SSRGPSPGLR PALPSRLARA SRGVDLATGP SRTPLRAKNK }\end{array}$

$\stackrel{130}{130}$ VHPSSTLEHH HHHH

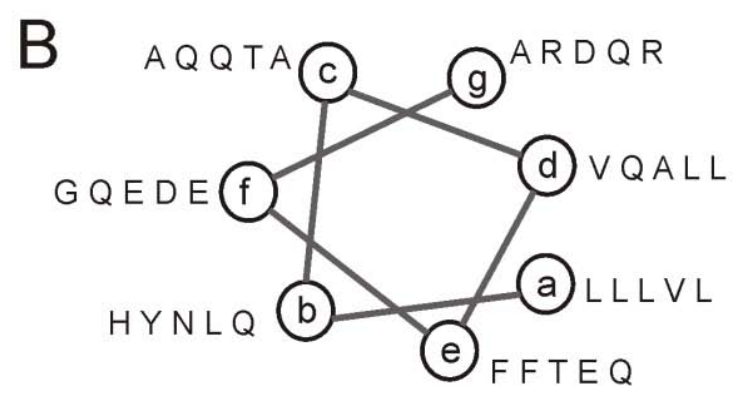

Fig. 4.4 (A) Sequence of the C-terminal portion of PC1 (134 amino acids) used in this work. Red letters indicate sequence elements derived from the original protein sequence, the coiled coil domain (yellow square) is presented as a helical wheel diagram (B).

Commonly often applied, solution based techniques to quantitatively investigate protein-protein interactions such as isothermal titration calorimetry (ITC) or fluorescence based techniques provide only thermodynamic, but no kinetic information or often require high protein concentration. Also, the introduction of fluorescent labels could affect the conformation an thus reactivity of the respective protein, which might alter the measured parameters.

These drawbacks were overcome by introducing surface coupled biosensors 5,6 , with which thermodynamic as well as kinetic data evaluation is possible in a time resolved manner and label free- manner (see chapter 1.2.1) Besides several techniques that are capable of detecting surface coupled binding events, the surface plasmon resonance (SPR) technique became the most commonly used method to study analyte-ligand interactions. ${ }^{7}$ The quartz crystal microbalance $(\mathrm{QCM})$ on the contrary is an acoustic biosensor that detects binding events as changes in resonance frequency and resistance of the quartz crystal resonator. $6,8,9$ Both the QCM and SPR technique are extremely sensitive gold coupled biosensors with a detection limit of $\sim 1 \mathrm{pM} / \mathrm{mm}^{2}$. 
To allow coupling of one interaction partner (ligand) on the biosensor surface, the gold surface has to be chemically modified. Therefore either planar, chemically modified alkanethioles $^{10}$ or three dimensional carboxylated dextran layers $(\sim 100-200 \mathrm{~nm})$ are used to specifically immobilize the ligand atop the surface (Fig. 4.5 A and B). ${ }^{11,5}$ Dextran surfaces are flexibly, non-crosslinked layers with reduced unspecific binding events that allow to homogenously immobilize a large amount of ligand within the three dimensional network. However, being immobilized on a two dimensional alkanethiol surface, a comparable accessibility of protein binding sites is guaranteed and diffusion processes are limited. ${ }^{5}$

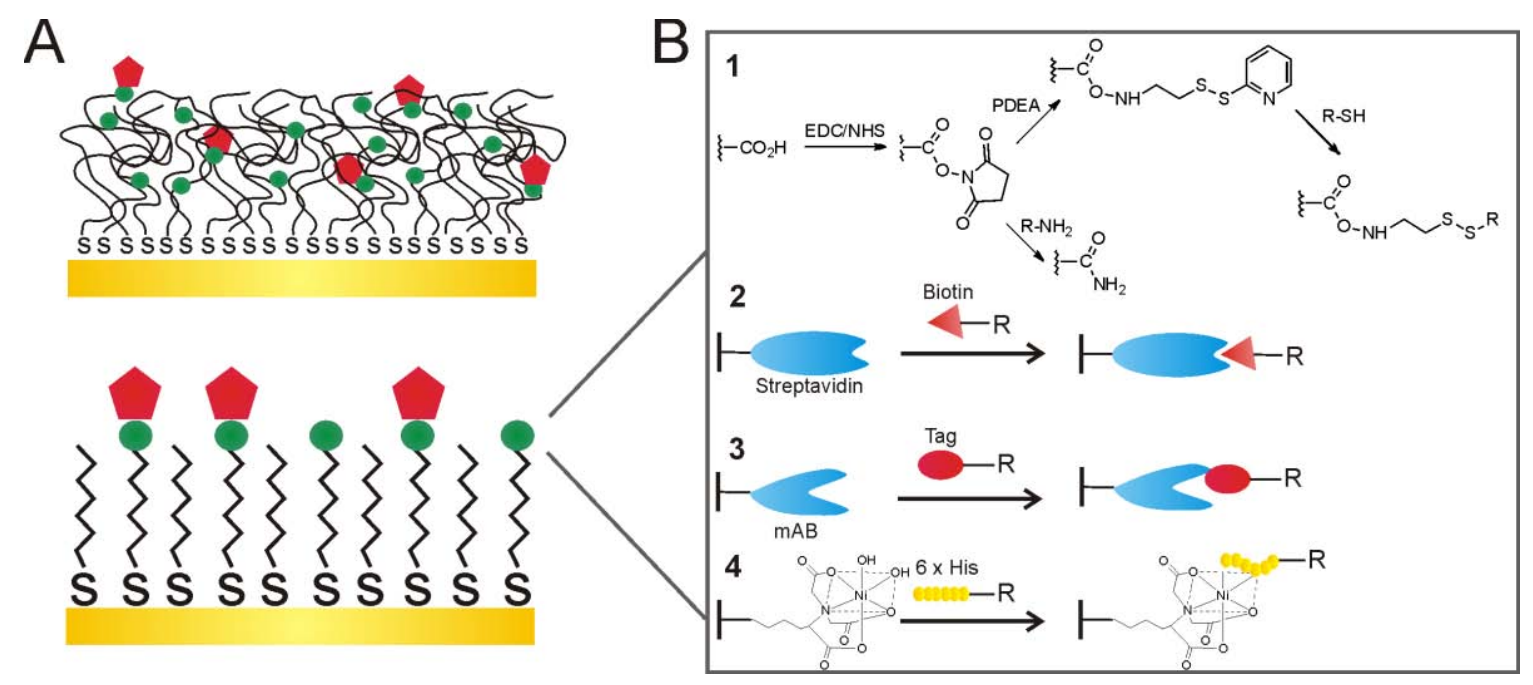

Fig. 4.5 (A) Scheme showing the three-dimensional structure of a dextran (upper part) and alkanethiol (lower part) covered gold surface (scales are not comparable). Dextrans and alkanethiols are chemically modified (green circles) to facilitate ligand (red pentagon) immobilization. (B) Coupling chemistry commonly used to immobilize ligands via amine or thiol coupling (1), streptavidin/biotin interaction (2), monoclonal antibody (mAB)/TAG (i.e. GST, HSV, FLAG) interaction (3) and Ni-NTA/hexahistidin $(6 \times$ His) interaction $(4)$.

Although in most biosensor applications one of the above mentioned immobilization strategies are utilized, solid supported lipid bilayers ${ }^{12}$ with specifically modified phospholipid head groups have the main advantage to mimic the cellular environment and maintain the lateral mobility of surface coupled proteins. Interactions of proteins with 
their respective lipid receptors such as the peanut agglutinin-GM1 ${ }^{13}$, annexin a1phosphatidylserine ${ }^{14}$ and ezrin- $\mathrm{PIP}_{2}{ }^{15}$ interaction were matter of investigation in the recent past.

As the adsorption of proteins on surfaces is governed and influenced by individual protein binding behaviors and biosensor setups, different kinetic models were developed to satisfy each distinct requirement. ${ }^{16}$ Important kinetic adsorption mechanisms and their corresponding model is depicted in Fig. 4.6
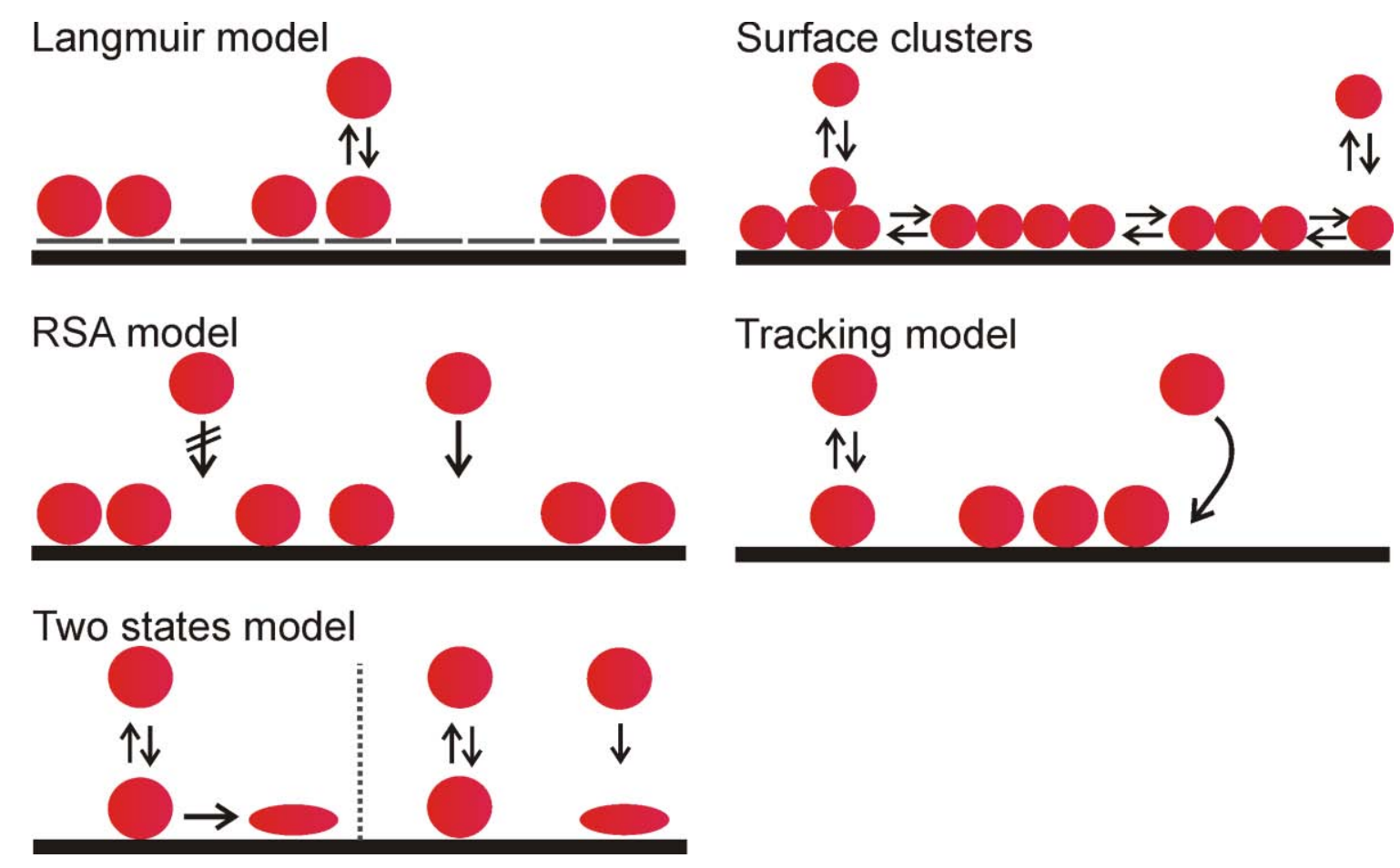

Fig. 4.6 Schematic drawing showing different kinetic models of protein adsorption. In the Langmuir model, adsorption takes place at distinct available surface sites, while in the RSA model the adsorption takes place on random available surface sites. The two states model refers to conformational changes upon adsorption leading to a higher affinity (left: transition model, right: two path model). Surface clusters can form either via the piggyback pathway (left) or lateral monomer diffusion (right). Cooperative adsorption takes place at the tracking model, where proteins preferably adsorb close to other surface bound proteins. ${ }^{16}$

The simplest model that describes the adsorption of small, rigid particles at distinct available surface sites has been proposed by Langmuir in 1932. This, non cooperative 
adsorption leads to the formation of a complete monolayer at a maximal surface coverage $\left(\Theta_{\max }=1\right)$. Moreover, the Langmuir model assumes that small, rigid particles adsorb on individual, independent binding sites with the same adsorption energy. This assumption however, is not valid for large, non-rigid proteins, which might cover more than one binding site. This weakness had been overcome by the development of the random sequential adsorption (RSA) model, at which the probability of protein binding is decreased at higher surface coverage, leading to a jamming limit of maximal surface coverage $\Theta_{\max } \approx 0.55 .{ }^{17}$ An exact description of the surface coverage can be obtained as a function of available surface sites (available surface function, $\Phi(\Theta)$ ). As the available surface function cannot be expressed analytically, other models such as the scaled particle theory (SPT, see section 3.4 .3 on kinetic data modeling) have to be adopted. ${ }^{18}$ In case of surface coupled interactions, mass transport of the respective particle from the bulk solution to the sensor surface is another factor that needs to be implemented in terms of a mass transport rate constant $k_{\text {tr. }}$. Although there are some possibilities to reduce mass transport effects, such as increase of the flow rate and lowering the surface concentration of the ligand, it can usually not be completely eliminated. ${ }^{19}$ If mass transport is influencing the binding kinetics of the interaction, both the rate constants of association $\left(k_{\mathrm{on}}\right)$ and dissociation $\left(k_{\mathrm{off}}\right)$ are distorted. ${ }^{20}$ While $k_{\mathrm{on}}$ is under-determined, due to lack of proteins in the so called unstirred layer directly above the sensor surface, $k_{\text {off }}$ is underdetermined due to rebinding of proteins to the sensor surface. Hence, kinetic models that take mass transport effects into account, leading to the extraction of a mass transport rate constant $k_{\mathrm{tr}}$, are often employed, if a detailed kinetic analysis is performed.

Other adsorption phenomenon are mainly associated with the protein characteristics. Lateral interaction, such as cooperative binding or protein clustering can influence the protein binding kinetics. ${ }^{15}$ Cooperative binding was first described by Minton and Chatelier, leading to a sigmoidal adsorption isotherm. ${ }^{21}$ Reorientation or conformational changes of proteins that have been adsorbed on a surface leading to a different binding behavior compared to proteins in solution, is also commonly observed. ${ }^{16}$ 
In this work, we aimed to establish a membrane based biosensor assay that would allow us to quantify the interaction between C-terminal polycystin-2 (cPC2, $\mathrm{Ile}^{680}-\mathrm{Val}^{986}$ ) and some of its potential interaction partners in a label-free and time-resolved manner. To be able to develop this biosensor assay, we used C-terminal PC1 (cPC1), which is an accepted interaction partner of $\mathrm{CPC} 2$, with a yet unknown affinity. A membrane based assay was chosen to, on the one hand provide the lateral mobility of the immobilized protein and, on the other hand mimic the cellular environment of the two membrane coupled protein fragments.

Therefore, the hexahistidine tagged protein CPC1 was specifically immobilized on a DOGS-NTA-Ni containing solid supported hybrid membrane. ${ }^{22}$ The cPC2-cPC1 interaction was investigated by means of either the QCM (set up is depicted in Fig. 4.7) or SPR biosensor and the protein-protein interaction was then analyzed using a kinetic model that based on the scaled particle theory in order to extract the dissociation constant $\left(K_{\mathrm{D}}\right)$ and rate constants of adsorption $\left(k_{\mathrm{on}}\right)$, desorption $\left(k_{\mathrm{off}}\right)$ and mass transport ( $\left.k_{\text {trans }}\right)$. After establishing this assay with the two model proteins cPC2 and cPC1, it should serve as a screening platform that allows to investigate the interactions that centre PC2 (see Fig. 4.1), varying possible parameters (see chapter 5 und 6). 


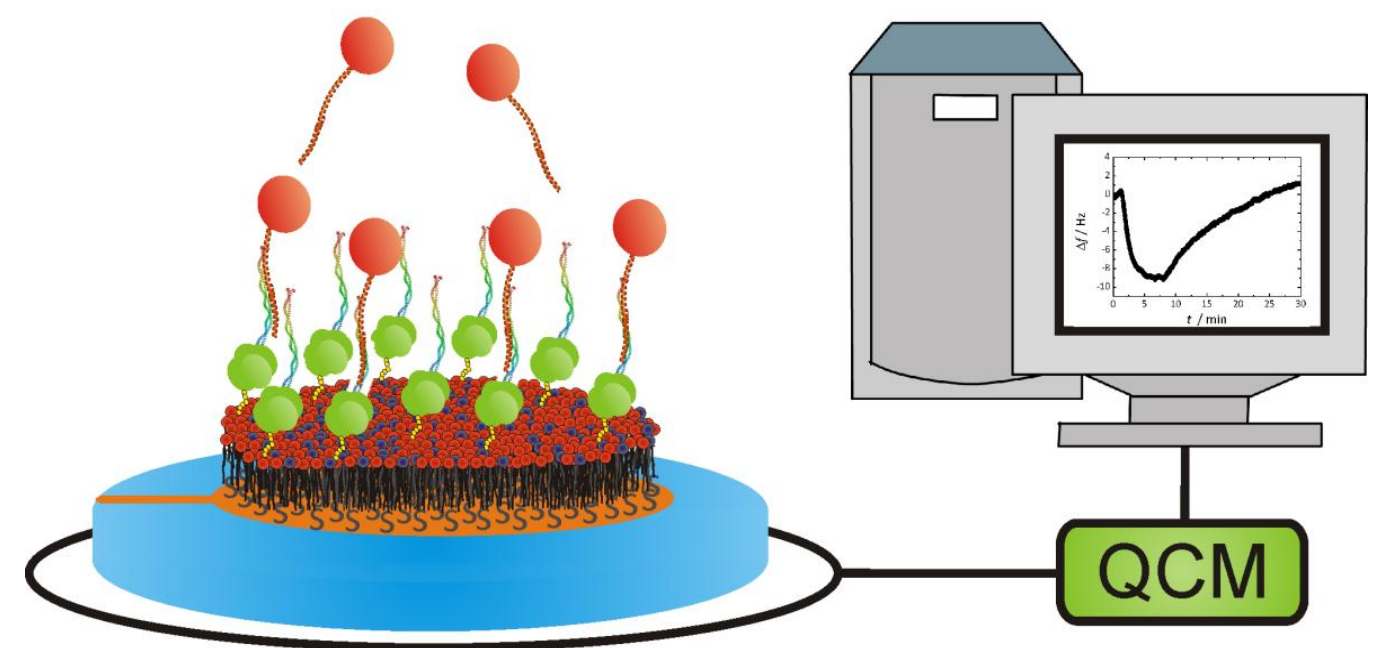

Fig. 4.7 Schematic drawing of the binding assay. cPC1 (shown in green) is immobilized on a solid supported membrane composed of octanethiol-DOPC/DOGS-NTA-Ni, 9:1 via its histidine tag (depicted in yellow). The solid supported membrane is prepared on a gold electrode of a $5 \mathrm{MHz}$ quartz crystal. cPC2 (shown in red) is added in solution and binding is monitored by changes in the resonance frequency of the quartz plate.

\subsection{Results}

\subsubsection{Protein Expression and Purification}

\section{C-Polycystin-1}

The C-terminal domain of polycystin-1 (cPC1, 136 amino acids, Ser ${ }^{4192}$-Thr ${ }^{4303}$, Fig. 4.4 A) was recombinantly expressed in Escherichia coli (strain: BL21(DE3)) with a hexahistidine tag at the C-terminus. The high purity of the protein solution after Niiminodiacetic (Ni-IDA) affinity purification was confirmed by SDS-PAGE analysis (Fig. 4.8, lane L and E). The gel shows CPC1 at approximately $14 \mathrm{kDa}$ (lane E). By means of Western Blot analysis the accessibility and functionality of the hexahistidine tag was confirmed (Fig. 4.8,WB, lane E) using a pentahistidine antibody from Qiagen (Hilden, Germany). 


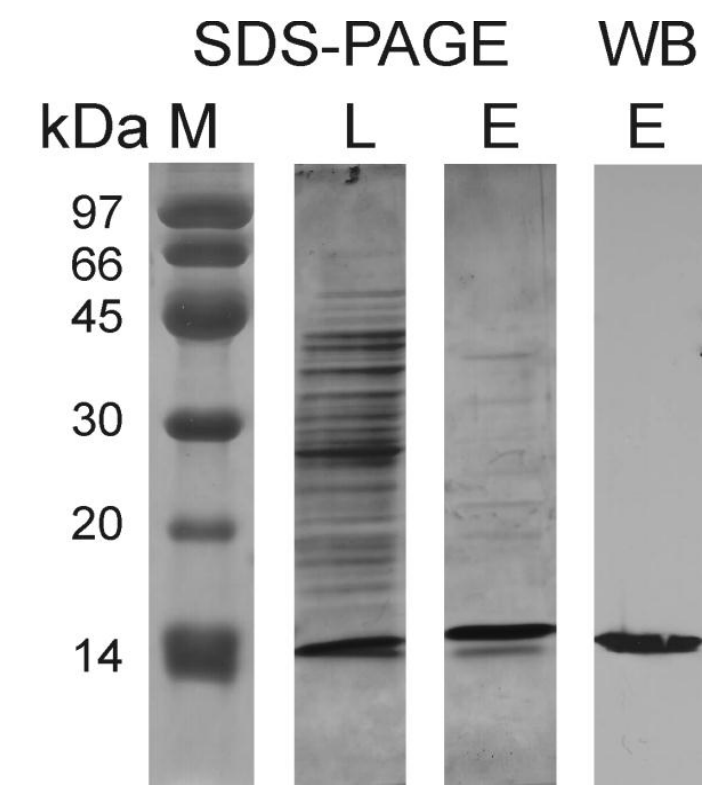

Fig. 4.8 SDS gels and Western blot analysis of C-Polycystin-1 ( $\mathrm{CPC1}$ ) protein fractions. The Coomassie stained SDS gel shows the molecular marker (M), cell lysate (L) and elution fraction (E) of cPC1. According to the SDS gel and Western blot analysis (WB, anti Hiss) the molecular weight of cPC1 is approximately $14 \mathrm{kDa}$.

\section{C-Polycystin-2}

The C-terminal fragment of polycystin-2 (cPC2, 290 amino acids, Ile $\left.{ }^{680}-\mathrm{Val}^{968}\right)$ was expressed and purified as a maltose binding protein (MBP) fusion protein to increase protein solubility and stability of $\mathrm{CPC} 2$ during all expression and the purification steps. A SDS-PAGE gel analysis of CPC2-MBP was performed after amylose affinity purification (compare Fig. 4.9, cPC2-MBP, lane L and E of cell lysate and elution fraction). The fusion protein was obtained with an approximate mass of $76 \mathrm{kDa}$. After TEV-protease cleavage, the cleaved protein was isolated from the MBP construct using a Niiminodiacetic (Ni-IDA) agarose column (see chapter 3.3.2). Due to internal histidine residues the affinity of $\mathrm{CPC} 2$ to the Ni-IDA column material was higher compared to that of the MBP. While the MBP was found exclusively in the flow through (FT) fraction (Fig. 4.9, cPC2 lane FT, M 40 kDa), the cleaved cPC2 fragment could be eluted with $30 \mathrm{~mm}$ imidazole (Fig. 4.9, cPC2, lane E, M $33 \mathrm{kDa}$ ). Compared to proteins that 
possess an artificial hexahistidine tag, the imidazole concentration was quite low, which denotes the lower affinity of $\mathrm{CPC} 2$ to the column material.

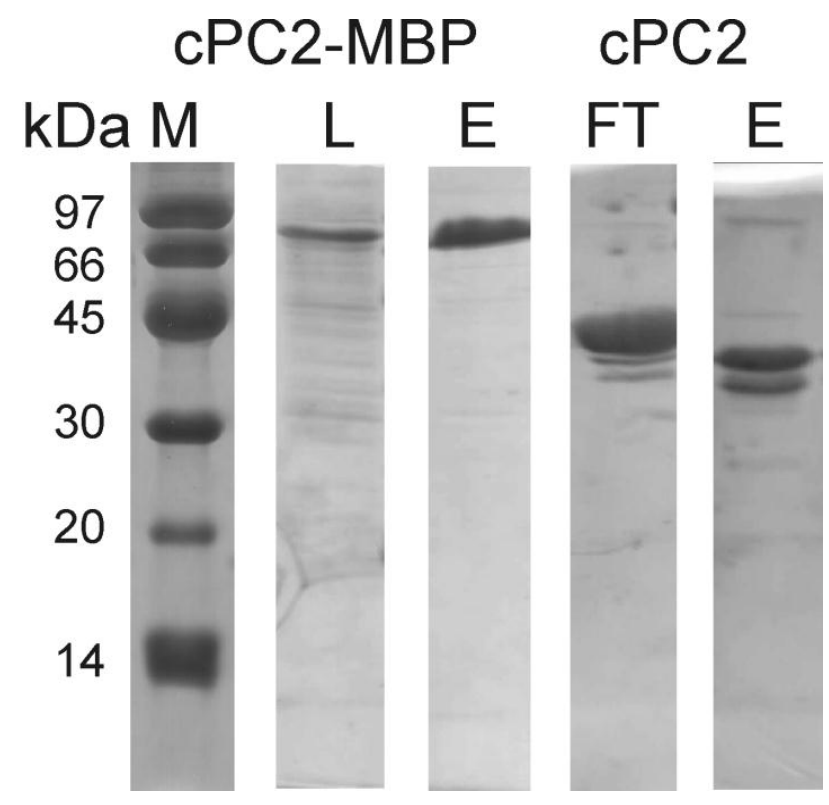

Fig. 4.9 SDS gels of cPC2-MBP as fusion protein and after TEV protease cleavage (cPC2). The Coomassie stained gel shows the molecular marker (M), the cell lysate $(C)$ and elution fraction (E) of the fusion protein cPC2-MBP with $76 \mathrm{kDa}$ (left hand side) and of cPC2 with $33 \mathrm{kDa}$ after cleavage from MBP (right hand side). The flow through (FT) after cleavage shows the MBP at $40 \mathrm{kDa}$.

\subsubsection{Impedance and SPR Analysis of Hybrid Solid Supported Mem- branes on Gold}

Solid supported hybrid membranes were prepared on gold as described in chapter 3.2.3. The lipid composition of the membranes was chosen so that specific coupling of the regarding protein of interest was possible (i.e. DOGS-NTA-Ni as a receptor lipid for hexahistidine tag proteins). As a defect free membrane was essential to prevent unspecific protein binding and thus to correctly determine binding constants and kinetic parameters of the protein-protein interaction, a high value was set on controlling the membrane quality and identifying optimal spreading conditions. Hence, in situ moni- 
toring of the vesicle spreading and thickness determination of the lipid monolayer by means of SPR as well as the impedance spectroscopic membrane characterization were employed to guarantee an adequate membrane quality.

\section{SPR Analysis of Solid Supported Hybrid Membrane Formation on Octanethiole Gold Surfaces}

Spreading of small unilamellar vesicles (SUVs) composed of either DOPC/DOGS-NTA$\mathrm{Ni}$ (9:1) or pure DOPC on a octanethiol-gold surface of an LaSFN9 glass plate was monitored in situ by means of the SPR in kinetic mode (see chapter 3.4.5, page 79). The shape of the curve as well as the monolayer thickness determined after the spreading process provides valuable information about the membrane quality i.e. the existence of defects or vesicles adsorbed on the surface.

The time cores of the relative reflectivity change of pure DOPC vesicle spreading performed in low salt E1 buffer ( $50 \mathrm{mM} \mathrm{KCl,} 20 \mathrm{mM}$ TRIS/HCl, $0.1 \mathrm{mM}$ EDTA, $0.1 \mathrm{mM}$ $\mathrm{NaN}_{3}, \mathrm{pH}$ 7.4) is depicted in (Fig. 4.10, black curve). After vesicle addition (A), the sudden increase of relative reflectivity indicated spreading of the vesicles. In the case of pure DOPC vesicles, however, a baseline that would demonstrate the formation of a monolayer atop the hydrophobic octanethiol surface was not observed. Instead, the curve increased steadily, most likely due to vesicle adsorption atop the monolayer surface. After rinsing with buffer (B), a small drop in relative reflectivity indicated the desorption of a few surface adhered vesicles. A membrane thickness of $d=3.9 \mathrm{~nm}(\mathrm{n}=1)$ could then be determined according to a procedure described in chapter 3.4.5 on page 81.

While spreading of DOPC/DOGS-NTA-Ni, 9:1 vesicles in high salt P1 buffer (500 mM NaCl, 20 mM TRIS/HCl, pH 8.0) was usually completed after 60-90 min with a mean thickness of $d=(3.4 \pm 1.6) \mathrm{nm}(\mathrm{n}=17$, Fig. 4.10, blue curve), spreading of identically composed vesicles in low salt $\mathrm{E} 1$ buffer in the presence of $10 \mathrm{mM} \mathrm{CaCl} 2$ resulted in the formation of a monolayer $(d=(1.2 \pm 0.5) \mathrm{nm}, \mathrm{n}=3)$ within $20-30 \mathrm{~min}$ (Fig. 4.10, red curve). 


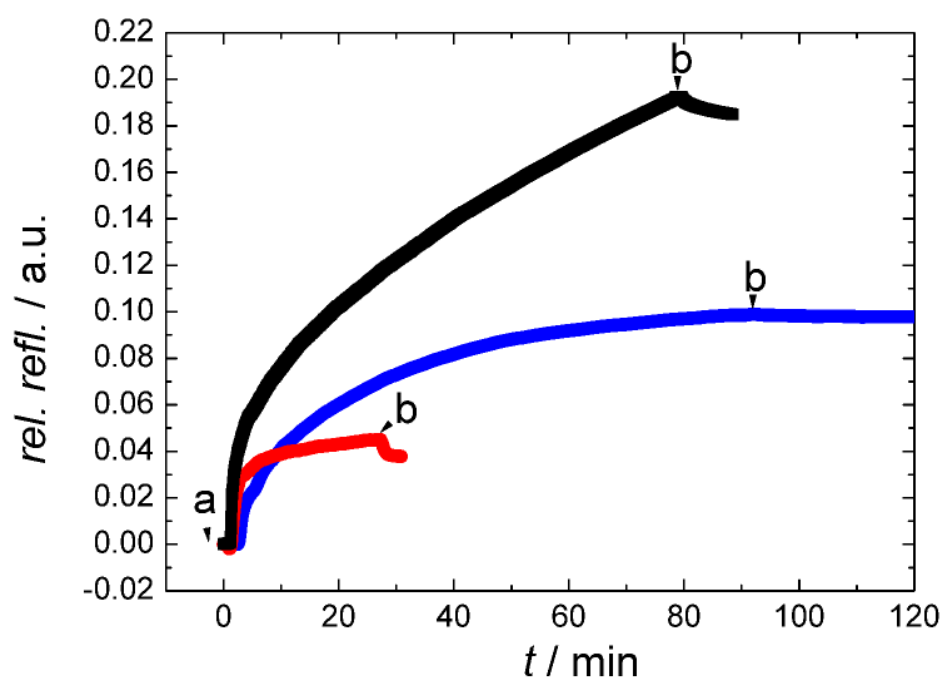

Fig. 4.10 Exemplary time courses of the relative reflectivity change after addition of SUVs (a) and buffer rinsing (b). DOPC SUVs (black curve) were added in low salt E1 buffer (50 mM KCl, $20 \mathrm{mM}$ TRIS/HCl, $0.1 \mathrm{~mm}$ EDTA, $0.1 \mathrm{mM} \mathrm{NaN}_{3}$, pH 7.4), while SUVs containing DOPC/DOGS-NTA-Ni (9:1) were applied in high salt P1 buffer (500 mM NaCl, $20 \mathrm{mM}$ TRIS/HCl, pH 8.0, blue curve) or low salt E1 buffer in the presence of $10 \mathrm{mM} \mathrm{CaCl}_{2}$ (red curve).

These values differ from the one expected for a perfect lipid monolayer $(d=2 \mathrm{~nm})$, a fact, that will be discussed in chapter 4.4. Despite the significant deviation in monolayer thickness, DOGS-NTA-Ni containing membranes formed under both high salt and low salt conditions were used to specifically immobilize hexahistidine tagged proteins. It was assumed that multilayers or vesicles adhered at the membrane surface would not interfere with the immobilization of proteins on the membrane.

\section{Impedance Analysis of an Octanethiol-gold Layer and a Solid Supported Membrane}

Both the octanethiol monolayer on gold coated AT-cut quartz crystals used for QCM experiments and the solid supported hybrid monolayer (DOPC/DOGS-NTA-Ni, 9:1) formed atop were characterized by means of impedance spectroscopy in order to ensure an appropriate monolayer coverage. Characteristic impedance spectra of both lay- 
ers are presented in Fig. 4.11 (octanethiole monolayer: hollow squares, bilayer: hollow circles). The Bode plots show the phase shift $\varphi(\omega)$ (blue squares and circles) and magnitude of impedance $|Z(\omega)|$ (black squares and circles) as a function of frequency (range: $f=10^{-1}-10^{6} \mathrm{~Hz}$ (see chapter 3.4.1).

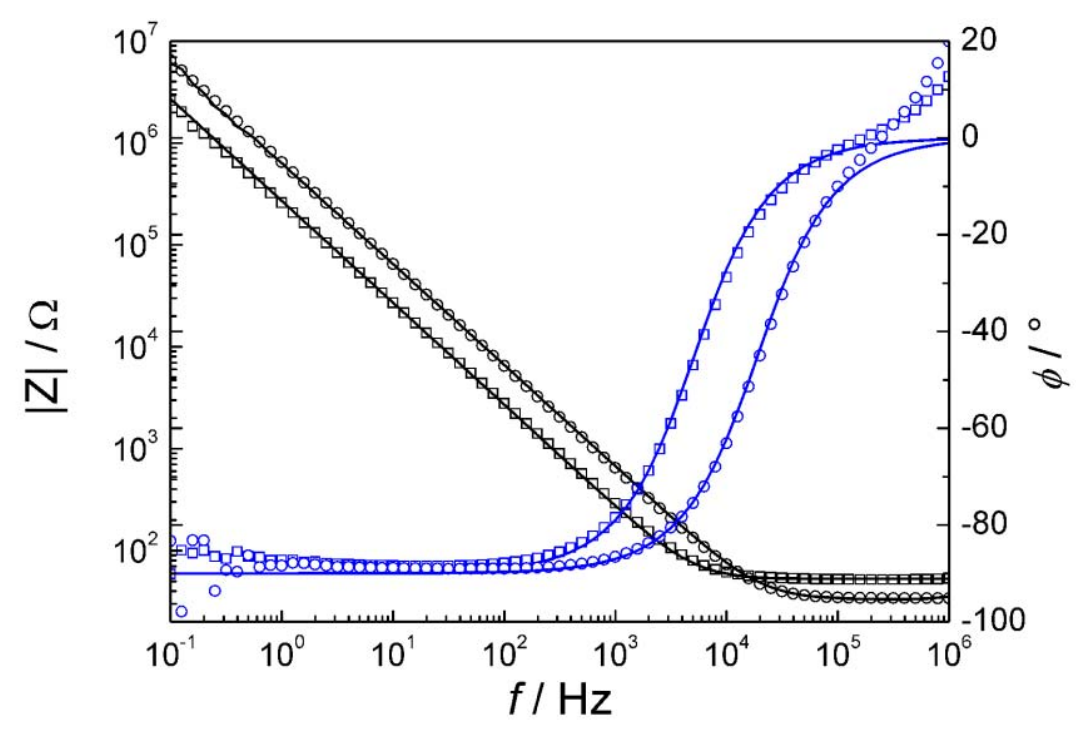

Fig. 4.11 Bode plot of the phase shift $\varphi(\omega)$ (blue squares and circles) and impedance $|\mathrm{Z}(\omega)|$ (black squares and circles) as a function of frequency (range: $f=10^{-1}-10^{6} \mathrm{~Hz}$ ). Impedance spectra of an octanethiol monolayer (hollow squares) and solid supported bilayer (hollow circles) were recorded and the parameters of a CR-equivalent circuit were fit to the data (black and blue lines).

Fitting the parameters of a CR-equivalent circuit to the data lead to a mean specific capacitance of Сот $=(2.3 \pm 0.2) \mu \mathrm{F} / \mathrm{cm}^{2} \quad(\mathrm{n}=27)$ for the octanethiol monolayer and $C_{\mathrm{BL}}=(1.1 \pm 0.1) \mu \mathrm{F} / \mathrm{cm}^{2}(\mathrm{n}=26)$ for the bilayer. These values are in very good agreement with results obtained previously. ${ }^{15}$ As the capacitances of each layer ad up reciprocally, the capacitance of the lipid monolayer can be calculated to $C_{\mathrm{ML}}=(2.1 \pm 0.5) \mu \mathrm{F} / \mathrm{cm}^{2}$ $(n=26)$. 


\subsubsection{Fluorescence Microscopy and FRAP Analysis of Hybrid Solid Supported Membranes on Silicon}

DOPC membranes doped with 0, 5, and 15 mol\% DOGS-NTA-Ni receptor lipid and $0.5 \mathrm{~mol} \%$ Bodipy-PC were prepared on silicon substrates that were hydrophobically functionalized with dodecyl trichlorosilane (DTS) resulting in hybrid lipid bilayers similar to those prepared on octanethiol-functionalized gold surfaces used for the QCM studies. A contact angle of approximately $\Theta_{\text {water }}=93^{\circ}$ points out the significantly increased hydrophobicity of the DTS functionalized surface compared to a hydrophilic silicon wafer that has a contact angle of $\Theta_{\text {water }}=44^{\circ}$ (Fig. 4.12). The values were in accordance with those reported in the literature for similar systems like alkanethiols and other hydrophobic surfaces. ${ }^{23,24}$

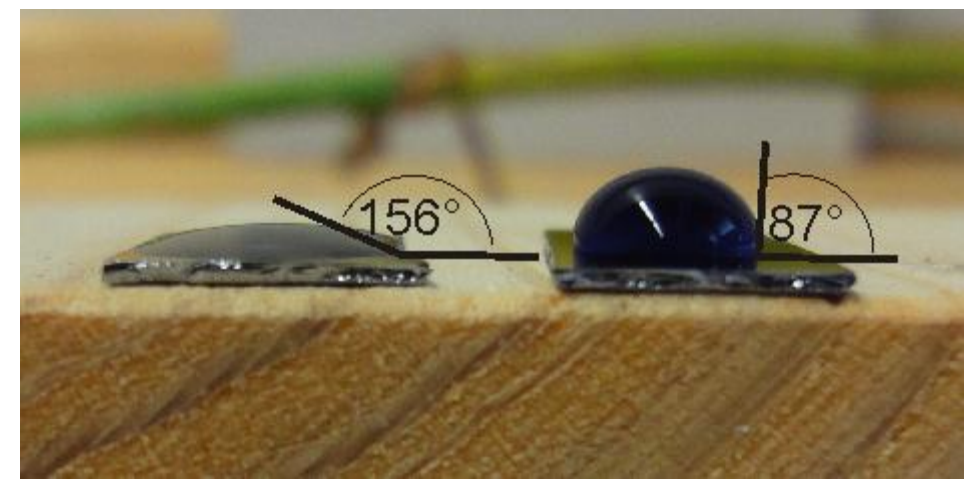

Fig. 4.12 Contact angle $\left(\Theta_{\text {water }}=180^{\circ}-\Theta\right)$ measurement of a water drop deposited on a hydrophilic silicon wafer (left hand side, $\Theta_{\text {water }}=93^{\circ}$ ) and a wafer that was hydrophobically functionalized with dodecyltrichlorosilane $\left(\Theta_{\text {water }}=44^{\circ}\right.$, right hand side).

Fluorescence microscopy images showed a homogeneous fluorescence indicating the successful formation of rather defect-free solid supported lipid bilayers independent of the DOGS-NTA-Ni content. Few non-spread vesicles were attached atop the membrane visible as bright spots. Fluorescence recovery after photobleaching (FRAP) analysis further corroborated the formation of continuous and fluid planar lipid bilayers with a 
mean diffusion coefficient of $D=(1.6 \pm 0.3) \mu \mathrm{m}^{2} \cdot \mathrm{s}^{-1}(\mathrm{n}=23)$, which was found to be independent of the DOGS-NTA-Ni content (Fig. 4.13). The immobile fraction was estimated to be $(26 \pm 9) \%$. The determined diffusion coefficient and immobile fraction is in the range of what has been found for hybrid solid supported membranes $\left(D=0.5-2 \mu \mathrm{m}^{2} \cdot \mathrm{s}^{-1} \text {, immobile fraction of about } 20 \%\right)^{35}$ and verifies the lateral mobility of the lipids independent of the DOGS-NTA-Ni content.

A

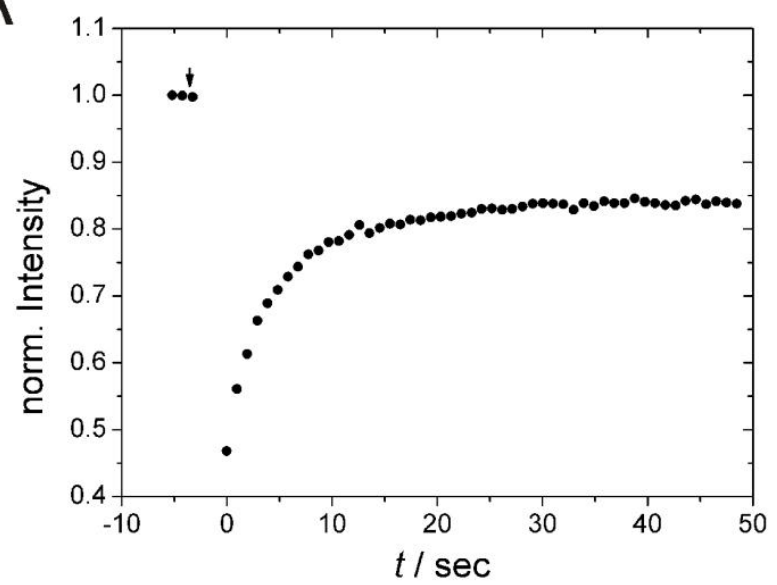

B
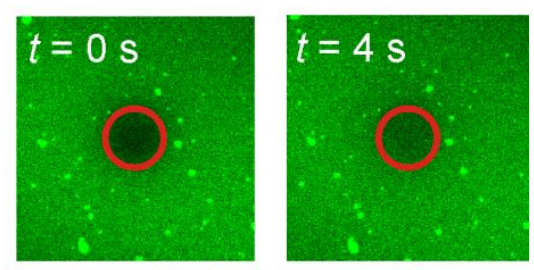

$t=40 \mathrm{~s}$

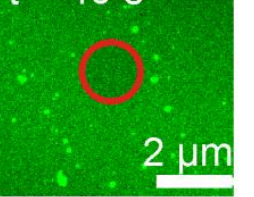

Fig. 4.13 (A) Exemplary FRAP curve obtained from solid supported hybrid membrane composed of DOPC/DOGS-NTA-Ni/Bodipy-PC, 84.5:15:0.5. The arrow indicates the time point of the bleaching pulse. (B) Exemplary fluorescence images observed after bleaching a region of interest (ROI) of the solid supported hybrid membrane.

\subsubsection{His6-tag Protein Immobilization on DOGS-NTA-Ni Containing Membranes}

In the next step, the immobilization of hexahistidine tagged proteins PIGEA14 and ezrin on DOGS-NTA-Ni containing membranes was investigated. Both QCM and SPR experiments were conducted to study the influence of the DOGS-NTA-Ni content on protein binding and the specificity of protein binding by means of an imidazole elution experiment. 
Immobilization of PIGEA14 on Solid Supported Membranes with Variable DOGS-NTA-Ni Content by Means of QCM

For QCM experiments solid supported DOPC membranes with different contents of the DOGS-NTA-Ni receptor lipid (0 mol\%, $2.5 \mathrm{~mol} \%, 5 \mathrm{~mol} \%, 7.5 \mathrm{~mol} \%, 10 \mathrm{~mol} \%$ and $15 \mathrm{~mol} \%$ DOGS-NTA-Ni) were prepared on the octanethiol-gold surface of an AT-cut quartz crystal as described in chapter 3.2.3.

In Fig. 4.14 A, typical time courses of the frequency shifts $(\Delta f)$ are shown after the addition of $0.3 \mu \mathrm{M}$ PIGEA14 to DOPC membranes with various DOGS-NTA-Ni contents. This protein concentration was chosen for all experiments, because a further increase of PIGEA14 concentration would not alter the resonance frequency. Hence, saturation of the binding sites with PIGEA14 was guaranteed. As the resonance frequency did not alter after addition of the hexahistidine tagged protein PIGEA14 to a pure DOPC membrane (reference experiment), unspecific protein adsorption was ruled out. Increasing the DOGS-NTA-Ni content from $2.5 \mathrm{~mol} \%$ to $5 \mathrm{~mol} \%$ and $7.5 \mathrm{~mol} \%$ lead to higher resonance frequency shifts at equilibrium $\left(\Delta f_{\mathrm{e}}\right)$. As a further increase of the DOGS-NTA-Ni content up to $15 \mathrm{~mol} \%$ did not significantly alter the measured frequency shifts $\Delta f_{e}$, is was concluded that a full surface coverage could be obtained at a minimum content of $\sim 7.5 \mathrm{~mol} \%$ DOGS-NTA-Ni. In Fig. $4.14 \mathrm{~B}$ the frequency shift $\Delta f_{\mathrm{e}}$ after protein addition is plotted as a function of DOGS-NTA-Ni content. The intercept of two linear fits defines the critical DOGS-NTA-Ni content of full protein coverage at approximately $7.5 \mathrm{~mol} \%$. DOGS-NTA-Ni. 

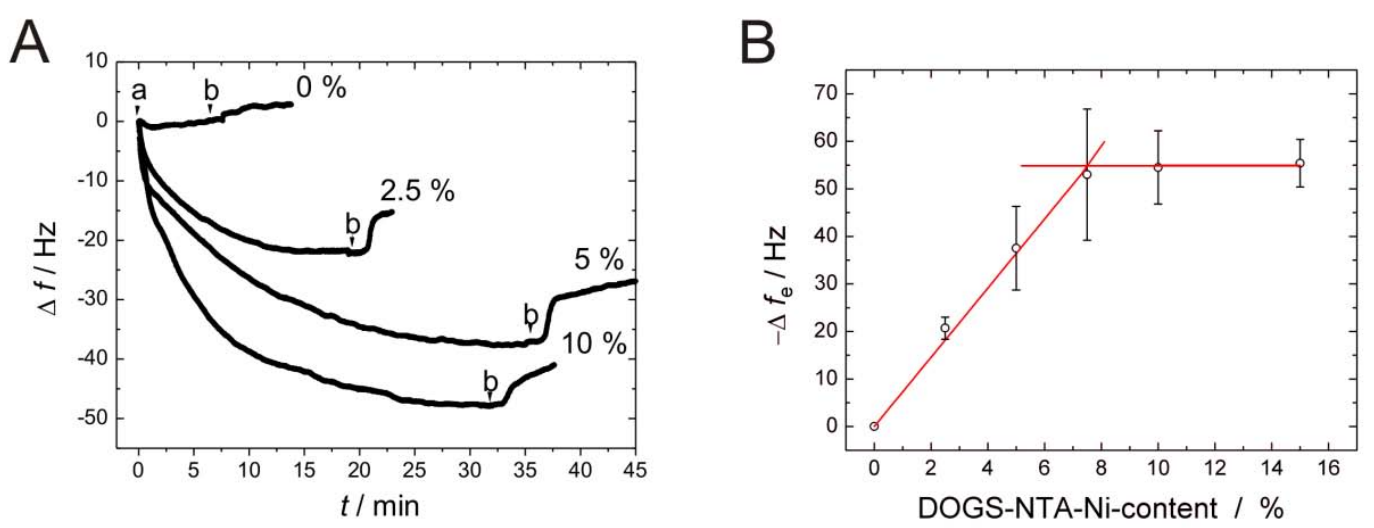

Fig. 4.14 (A) Exemplary time courses of the frequency shifts $(\Delta f)$ after the addition of $0.3 \mu \mathrm{M}$ PIGEA14 (a) to solid supported membranes composed of DOPC and different DOGS-NTA-Ni contents in high salt P1 buffer (500 mM NaCl, 20 mM TRIS/HCl, pH 8.0). Rinsing with buffer is indicated by (b). (B) Mean equilibrium frequency shifts $\left(-\Delta f_{e}\right)$ after the adsorption of $0.3 \mu \mathrm{M}$ PIGEA 14 to membranes composed of DOPC and various DOGS-NTA-Ni contents.

Mean values of $-\Delta f_{\mathrm{e}}$ after PIGEA14 addition to DOPC membranes with various DOGSNTA-Ni contents are listed in Table 4.1.

Table 4.1 Mean values of $-\Delta f_{\mathrm{e}}$ after addition of $0.3 \mu \mathrm{M}$ PIGEA14 to DOPC membranes with different DOGS-NTA-Ni contents.

\begin{tabular}{c|c} 
DOGS-NTA-Ni content $/ \mathbf{m o l} \%$ & $-\Delta f_{\mathrm{e}} / \mathbf{H z}$ \\
\hline $\mathbf{2 . 5}$ & $(21 \pm 2), \mathrm{n}=3$ \\
\hline $\mathbf{5}$ & $(38 \pm 9), \mathrm{n}=5$ \\
\hline $\mathbf{7 . 5}$ & $(53 \pm 14), \mathrm{n}=5$ \\
\hline $\mathbf{1 0}$ & $(54 \pm 8), \mathrm{n}=4$ \\
\hline $\mathbf{1 5}$ & $(55 \pm 5), \mathrm{n}=2$
\end{tabular}


However, all experiments that required a completely protein covered membrane were performed with $10 \mathrm{~mol} \%$ DOGS-NTA-Ni to reproducibly ensure full protein coverage.

Specific Binding and Elution of Ezrin on and from DOGS-NTA-Ni Containing Membranes Investigated by Means of SPR

The specific binding of hexahistidine tagged proteins to DOGS-NTA-Ni containing membranes was further investigated by means of SPR spectroscopy. This was to prove that both affinity constants and kinetic rate constants can bedetermined by means of the QCM technique as well as the optical SPR method. Besides this, experiments were performed with the hexahistidin tagged protein ezrin (for further information see chapter 7.2, page 196) to ensure that binding specifity is independent of the chosen protein. First, specific binding of ezrin to the receptor lipid DOGS-NTA-Ni was investigated by comparing the binding of $0.8 \mu \mathrm{M}$ ezrin to a solid supported hybrid membrane composed of DOPC containing 10 mol\% DOGS-NTA-Ni (Fig. $4.15 \mathrm{~A}$, red curve) and a pure DOPC membrane (Fig. 4.15 A, black curve). This protein concentration guaranteed complete saturation of all available binding sites. ${ }^{25}$ It turned out that the content of unspecifically bound ezrin to a pure DOPC membrane constitutes $14 \%$ of the ezrin bound to a membrane with $10 \mathrm{~mol} \%$ DOGS-NTA-Ni content. As this was a non negligible content, a further experiment was conducted to proof that ezrin binds only specifically to a $10 \mathrm{~mol} \%$ containing DOGS-NTA-Ni membrane (Fig. $4.15 \mathrm{~B}$ ). Ezrin, that was immobilized on a DOPC membrane containing 10 mol\% DOGS-NTA-Ni was firstly rinsed with low salt E1 buffer ( $50 \mathrm{~mm} \mathrm{KCl}, 20 \mathrm{mM}$ TRIS/HCl, $0.1 \mathrm{~mm}$ EDTA, $0.1 \mathrm{~mm} \mathrm{NaN}$, pH 7.4) to allow for desorption of unspecifically bound protein. Then, the system was rinsed with ezrin elution buffer ( $250 \mathrm{mM}$ imidazole, $50 \mathrm{mM} \mathrm{KCl,} 20 \mathrm{mM}$ TRIS/HCl, pH 7.4) which lead to the complete elution of ezrin from the membrane surface. 

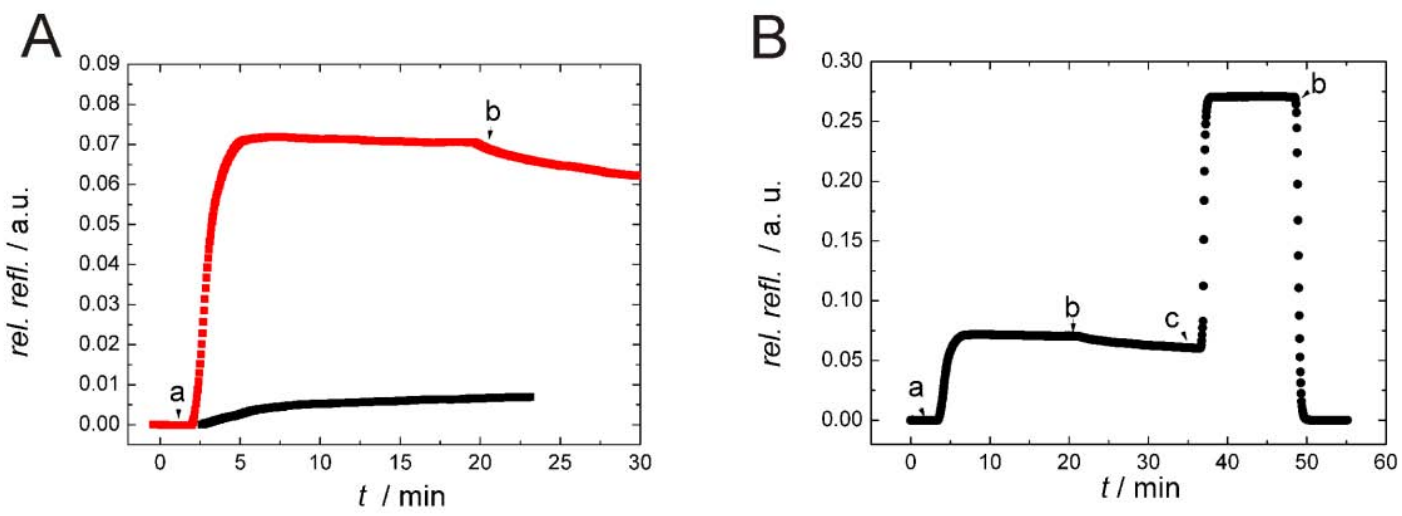

Fig. 4.15 (A) Exemplary time course of the relative reflectivity shift after the addition of $0.8 \mu \mathrm{M}$ ezrin (a) to a membrane composed of DOPC/DOGS-NTA-Ni, 9:1 (red curve) and a pure DOPC membrane (black curve). Rinsing with low salt E1 buffer ( $50 \mathrm{mM} \mathrm{KCl}, 20 \mathrm{mM}$ TRIS/HCl, $0.1 \mathrm{mM}$ EDTA, $0.1 \mathrm{mM} \mathrm{NaN}$, $\mathrm{pH}$ 7.4) is indicated by (b). (B) Exemplary time course of the relative reflectivity shift after the addition of $0.8 \mu \mathrm{M}$ ezrin (a) to a membrane composed of DOPC/DOGS-NTA-Ni, 9:1. Rinsing with E1 buffer (b) only led to desorption of unspecifically bound ezrin, while rinsing with a $250 \mathrm{mM}$ imidazole buffer $(250 \mathrm{mM}$ imidazole, $50 \mathrm{mM} \mathrm{KCl}, 20 \mathrm{~mm}$ TRIS/HCl, $\mathrm{pH}$ 7.4) (c) lead to the specific elution of ezrin.

This effect could only be obesevred after rinsing with E1 buffer, which readjusted the starting refractive index conditions necessary to determine the amount of eluted ezrin. It turned out that $100 \%$ of the ezrin bound to the DOGS-NTA-Ni containing membrane was eluted from the membrane surface. Thus, it was proven hexahistidine tagged proteins specifically bind to DOPC membranes with a 10 mol\% DOGS-NTA-Ni content. Both biosensor methods the SPR and the QCM technique are appropriate to investigate this protein-membrane interaction.

\subsubsection{Interaction of cPC1 and cPC2 on Solid Supported Membranes In- vestigated by Means of QCM and SPR}

After the formation of solid supported hybrid membranes composed of DOPC/DOGSNTA-Ni, 1:9 with specifically bound hexahistidine tagged $\mathrm{cPC} 1$, the aim was to quantitatively investigate the interaction of $\mathrm{CPC} 1$ with its putative interaction partner $\mathrm{CPC} 2$. It was important that all available DOGS-NTA-Ni binding sites were saturated with cPC1 
(or other binding partners), as cPC2 binds to DOGS-NTA-Ni most likely via internal histidine residues. Thus, saturation of the binding sites with CPC1 was ensured by adding as much $\mathrm{CPC} 1$ to the system until no more change of the measured signal indicated further binding to the membrane.

Both the optical SPR technique as well as the acoustic QCM method were applied in order to examine, whether both techniques are equally appropriate to investigate the protein-protein interaction in a quantitative manner. First, specific binding of $\mathrm{cPC} 2$ to cPC1 immobilized on a DOPC/DOGS-NTA-Ni, 9:1 membrane was investigated by the QCM technique. Fig. 4.16 A shows a characteristic time course of the frequency shift after the addition of $0.22 \mu \mathrm{M}$ cPC2 in $\mathrm{Ca}^{2+}$ containing buffer $(150 \mathrm{mM} \mathrm{NaCl}, 20 \mathrm{mM}$ HEPES, $1 \mathrm{mM} \mathrm{CaCl}$, $\mathrm{pH} 7.4$ ) that led to a frequency shift of $\Delta f_{\mathrm{e}}=-10.5 \mathrm{~Hz}$. Rinsing with buffer lead to a complete cPC2 desorption indicated by an increase in resonance frequency to a value of $\Delta f_{\mathrm{e}}=0 \mathrm{~Hz}$. This demonstrates an almost fully reversible interaction in this experiment. A mean value of $(66 \pm 13) \%, n=5$ was determined as the content of cPC2 desorption. The same experiment was then performed by means of the SPR technique. Fig. $4.16 \mathrm{~B}$ shows an exemplary time course of the relative reflectivity change after the addition of $0.3 \mu \mathrm{M} \mathrm{CPC} 2$ to $\mathrm{CPC} 1$ that was immobilized on a solid supported hybrid DOPC membrane doped with 10 mol\% DOGS-NTA-Ni. An immediate increase of relative reflectivity of rel.refl. $=0.011$ corresponding to a thickness of $d=0.7 \mathrm{~nm}$ (see chapter 3.4.5, page 81 for details regarding the thickness calculation). After rinsing with buffer only $40 \%$ of the bound $\mathrm{CPC} 2$ desorbed from the surface. A mean value of $(36 \pm 16) \%, n=7$ was determined as the content of desorbed proteins after buffer rinsing during the SPR experiments. 
A

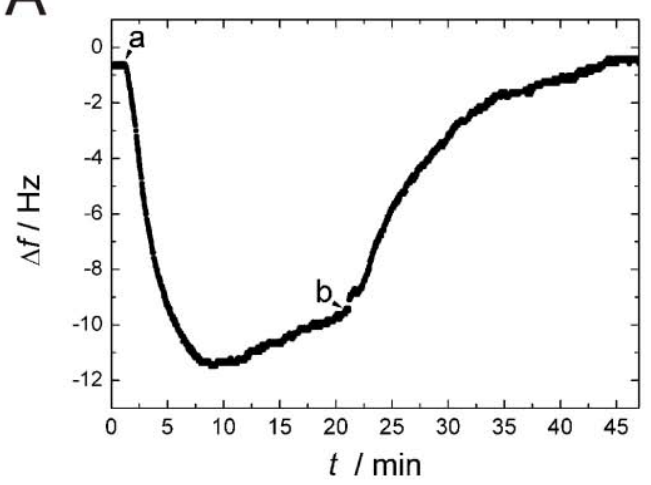

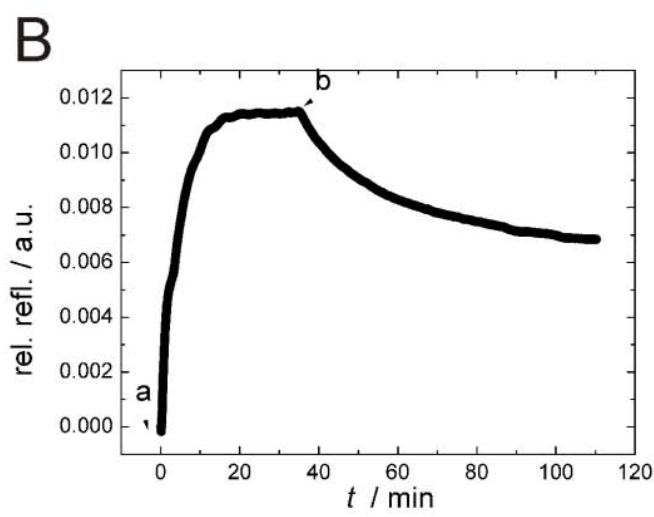

Fig. 4.16 (A) Representative time course of the frequency shift $(\Delta f)$ after addition of $0.22 \mu \mathrm{M} \mathrm{CPC} 2$ (a) to a DOPC/DOGS-NTA-Ni, 9:1 membrane with bound cPC1 in $\mathrm{Ca}^{2+}$ containing buffer $(150 \mathrm{mM} \mathrm{NaCl}, 20 \mathrm{mM}$ HEPES, $1 \mathrm{mM} \mathrm{CaCl}$, pH 7.4); (b) indicates the time point of buffer rinsing. (B) Exemplary time course of the relative reflectivity shift after addition of $0.30 \mu \mathrm{M} \mathrm{CPC2}$ (a) to a DOPC/DOGS-NTA-Ni, 9:1 membrane covered with $\mathrm{cPC} 1$ in $\mathrm{Ca}^{2+}$ containing buffer.

The mean thickness of cPC2 bound to $\mathrm{CPC} 1$ considering only $\mathrm{cPC} 2$ concentration that ensure saturation of all available binding sites $\left(c_{\mathrm{CPC} 2}>0.3 \mu \mathrm{M}\right)$ was determined to $d=(0.6 \pm 0.2) \mathrm{nm}(\mathrm{n}=5)$.

\subsubsection{Binding Affinity Determination}

In order to quantify the interaction of cPC2 with $\mathrm{cPC} 1$ immobilized on a membrane composed of DOPC/DOGS-NTA-Ni, 9:1 in $\mathrm{Ca}^{2+}$ containing buffer (150 mM NaCl, $20 \mathrm{mM}$ HEPES, $\left.1 \mathrm{mM} \mathrm{CaCl}_{2}, \mathrm{pH} 7.4\right)$ the resonance frequency shift $\left(\Delta f_{\mathrm{e}}\right)$ and the relative reflectivity change was plotted versus the corresponding cPC2 concentration (Fig. 4.17). The parameters of a simple Langmuir adsorption isotherm (eq. 4.1) were fitted to the data:

$$
\Delta x=\Delta x_{\max } \frac{c_{\mathrm{cPC} 2}}{K_{\mathrm{D}}+c_{\mathrm{cPC} 2}},
$$


with $\Delta x$ being the respective measured variable either $\Delta f_{\mathrm{e}}$ or the thickness change $d$, $\Delta x_{\max }$ as the corresponding maximal value and $K_{\mathrm{D}}$ as the dissociation constant of the cPC2/cPC1 interaction. As the binding affinity of large proteins in complex cannot be correctly described by means of the Langmuir isotherm (see chapter 4.2), it was only applied in this case in order to compare the result obtained by the two techniques, not to provide correct thermodynamic information.

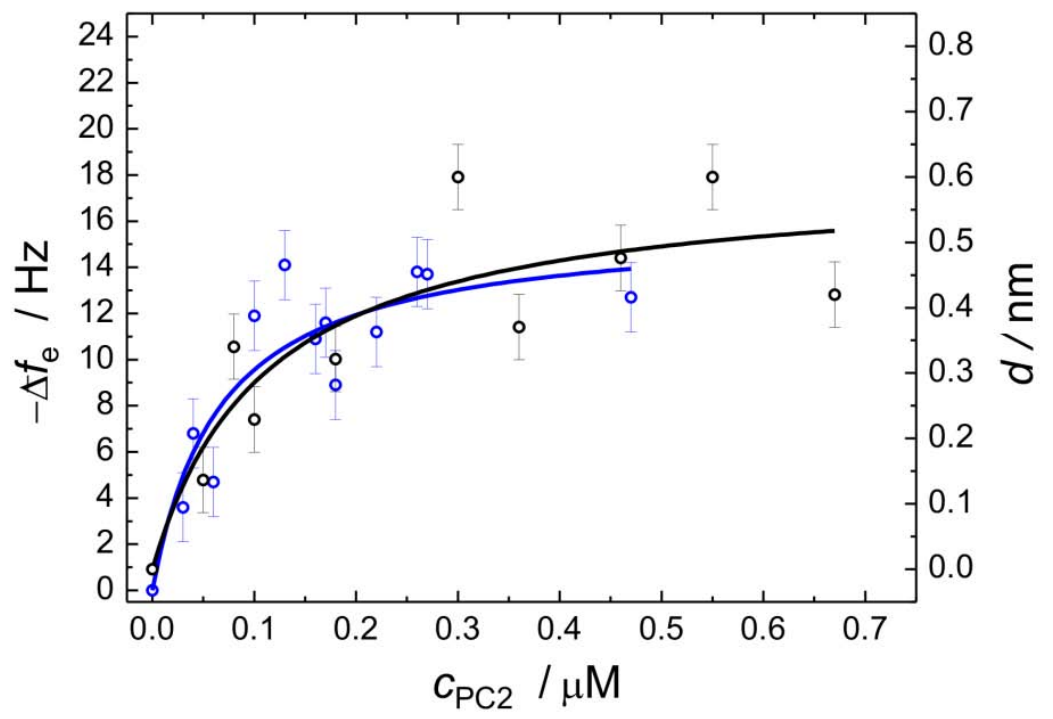

Fig. 4.17 Adsorption isotherms of the $\mathrm{cPC} 2 / \mathrm{CPC} 1$ interaction in $\mathrm{Ca}^{2+}$ containing buffer $(150 \mathrm{mM} \mathrm{NaCl}$, $20 \mathrm{mM}$ HEPES, $1 \mathrm{mM} \mathrm{CaCl}$, $\mathrm{pH}$ 7.4). The parameters of a Langmuir adsorption isotherm were fitted to the resonance frequency shifts $\left(-\Delta f_{\mathrm{e}}\right)$ obtained from QCM data (blue circles, blue line) and to the thickness change ( $d$ ) obtained from SPR experimental data (black circles, black line). A dissociation constant of $K_{\mathrm{D}}=(66 \pm 28) \mathrm{nM}$ was determined from $\mathrm{QCM}$ data $\left(\Delta f_{\max }=(16 \pm 2) \mathrm{Hz}\right)$, while from the obtained SPR data a dissociation constant of $K_{\mathrm{D}}=(92 \pm 53) \mathrm{nM}$ was determined $\left(d_{\max }=(0.6 \pm 0.1) \mathrm{nm}\right)$.

In case of the QCM experiments, a dissociation constant of $K_{\mathrm{D}}=(66 \pm 28) \mathrm{nM}$ and maximal shift of resonance frequency of $\Delta f_{\max }=(16 \pm 2) \mathrm{Hz}$ was determined, while the experimental data derived from SPR experiments resulted in a $K_{\mathrm{D}}=(92 \pm 52) \mathrm{nM}$ and a maximal thickness change of $d_{\max }=(0.6 \pm 0.1) \mathrm{nm}$. Both dissociation constants are basically identical taking the experimental error into account. 


\subsubsection{Kinetic Data Analysis}

\section{Langmuir Model}

Besides the dissociation constant $K_{\mathrm{D}}$ of the $\mathrm{CPC} 2-\mathrm{cPC} 1$ interaction, kinetic rate constants of the adsorption $\left(k_{\mathrm{on}}\right)$ and desorption $\left(k_{\mathrm{off}}\right)$ process were determined using the Langmuir model. Therefore, the time course of the frequency shift $(\Delta f)$ is fitted monoexponentially (eq. 4.2) assuming rate-limiting kinetics and neglecting the influence of mass transport:

$$
\Delta f(t)=\Delta f_{\mathrm{e}}\left(1-\exp \left(-k_{s} t\right)\right)
$$

with $\Delta f_{\mathrm{e}}$ being the equilibrium frequency.

An exemplary time course of the frequency shift, after the addition of $0.18 \mu \mathrm{M}$ cPC2 to cPC1 immobilized on a DOPC/DOGS-NTA-Ni, 9:1 membrane, along with the result of the monoexpoential fit $\left(k_{\mathrm{s}}=(8.20 \pm 0.03) \cdot 10^{-3} \mathrm{~s}^{-1}\right.$. $)$ is depicted in Fig. 4.18 . 


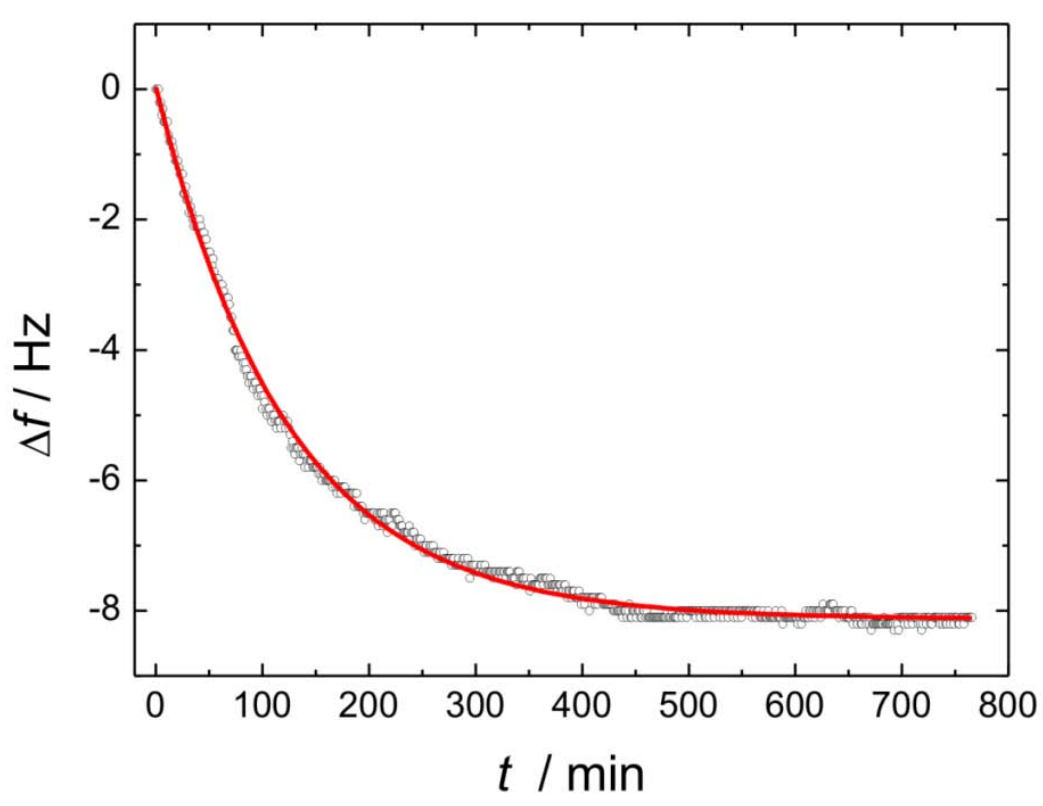

Fig. 4.18 Time course of the frequency shift after the addition of $0.18 \mu \mathrm{M} \mathrm{cPC} 2$ to a DOPC/DOGS-NTA$\mathrm{Ni}$, 9:1 membrane covered with cPC1 in $\mathrm{Ca}^{2+}$ containing buffer $(150 \mathrm{mM} \mathrm{NaCl}, 20 \mathrm{mM}$ HEPES, $1 \mathrm{mM}$ $\mathrm{CaCl}_{2}, \mathrm{pH}$ 7.4). The concentration dependent rate constant of protein adsorption $\left(k_{\mathrm{s}}\right)$ was fitted to the dat by means of a monoexponential fit. The rate constant was determined to $k_{\mathrm{s}}=(8.20 \pm 0.03) \cdot 10^{-3} \mathrm{~s}^{-1}$.

The linear relation between the concentration dependent rate constant $k_{\mathrm{s}}$ and the cPC2 concentration $\left(\mathcal{C}_{\mathrm{CPC} 2}\right)$ can be used to determine both $k_{\text {on }}$ and $k_{\text {off }}$ via a linear regression (eq. 4.3):

$$
k_{\mathrm{s}}=k_{\mathrm{on}} \cdot c_{\mathrm{cPC} 2}+k_{\mathrm{off}},
$$

In Fig. 4.19, $k_{\mathrm{s}}$ is plotted as a function of cPC2 concentration for results obtained by means of SPR (black circles) and QCM (blue circles) measurements. 


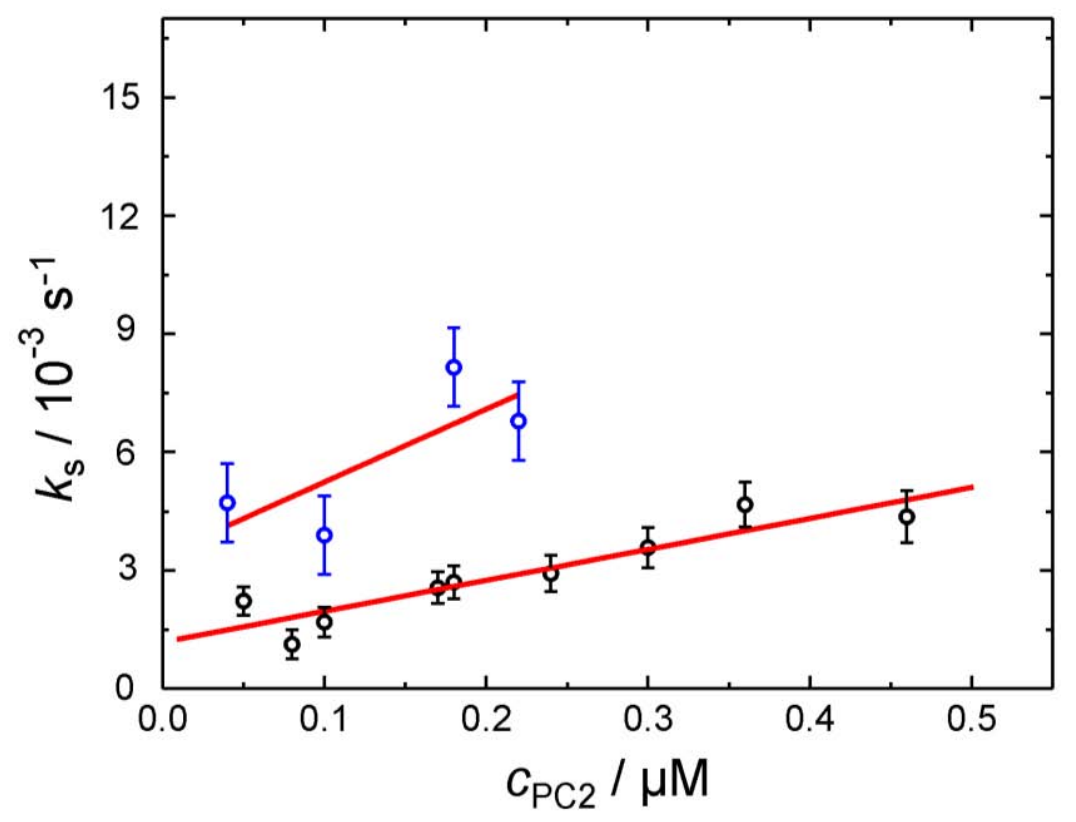

Fig. 4.19 Concentration dependent rate constants $\left(k_{s}\right)$ of $\mathrm{CPC} 2$ adsorption to $\mathrm{cPC} 1$ immobilized on a DOPC/DOGS-NTA-Ni, 9:1 membrane as a function of $\mathrm{CPC} 2$ concentration $\left(\mathcal{C}_{\mathrm{CPC}}\right)$ in $\mathrm{Ca}^{2+}$ containing buffer (150 mM NaCl, $20 \mathrm{mM}$ HEPES, $1 \mathrm{mM} \mathrm{CaCl}$, pH 7.4). From SPR measurements (black circles) values of $k_{\text {on }}=(7.9 \pm 1.2) \cdot 10^{-3} \mathrm{M}^{-1} \cdot \mathrm{s}^{-1}$ and $k_{\text {off }}=(1.2 \pm 0.3) \cdot 10^{-3} \mathrm{~s}^{-1}$ were determined, resulting in a $K_{\mathrm{D}}=(150 \pm 46) \mathrm{nM}$. From QCM measurements (blue circles) values of $k_{\text {on }}=(1.8 \pm 1.1) \cdot 10^{-2} \mathrm{M}^{-1} \cdot \mathrm{s}^{-1}$ and $k_{\text {off }}=(3.4 \pm 1.7) \cdot 10^{-3} \mathrm{~s}^{-1}$ were determined, resulting in a $K_{\mathrm{D}}=(180 \pm 140) \mathrm{nM}$.

In case of the SPR experiments the rate constants of association and dissociation were determined to $k_{\text {on }}=(7.9 \pm 1.2) \cdot 10^{-3} \mathrm{M}^{-1} \cdot \mathrm{s}^{-1}$ and $k_{\text {off }}=(1.2 \pm 0.3) \cdot 10^{-3}{ }^{1} \mathrm{~s}^{-1}$, while for the QCM experiment they were determine to $k_{\text {on }}=(1.8 \pm 1.1) \cdot 10^{-2} \mathrm{M}^{-1} \cdot \mathrm{s}^{-1}$ and $k_{\text {off }}=(3.4 \pm 1.7) \cdot 10^{-3} \mathrm{~s}^{-1}$. Comparing the course of the plots and the respective linear regression, it becomes obvious that they notably differ, although the determined kinetic rate constants are similar taking the error into account. This issue is discussed in detail in chapter 4.4. Applying eq. 4.4 , we were able to calculate the dissociation constant $K_{\mathrm{D}}$.

$$
K_{\mathrm{D}}=\frac{k_{\mathrm{off}}}{k_{\mathrm{on}}}
$$


A dissociation constants of $K_{\mathrm{D}}=(150 \pm 46) \mathrm{nM}$ was thus calculated from the values obtained by SPR measurements and $K_{\mathrm{D}}=(180 \pm 140) \mathrm{nM}$ from QCM measurements, respectively. These values significantly differ from those determined by means of the Langmuir adsorption isotherm (SPR: $\left.K_{\mathrm{D}}=(92 \pm 53) \mathrm{nM}, \mathrm{QCM} K_{\mathrm{D}}=(66 \pm 28) \mathrm{nM}\right)$. A deviation of the dissociation constants determined by means of an isotherm and applying the kinetic rate constants can provide an indication of wrongly determined kinetic rate constants. Mass transport of the proteins of the sensor surface, an effect that often arouses when adsorption phenomena are studied ad solid interfaces (see chapter 4.2), could cause this deviation. Hence, we decided to change the evaluation of the experimental data in order to obtain accurate kinetic rate constants. The kinetic model that bases on the scaled particle (see chapter 4.2) and implements the evaluation of the mass transport rate constant $k_{\text {tr }}$ seems to fulfill the requirements needed, if protein adsorption takes place at a solid interface.

\section{Model Based on the Scaled Particle Theory}

Due to the fact that the Langmuir model seemed insufficient to quantify the interaction of $\mathrm{CPC} 2$ with $\mathrm{cPC} 1$, the rate constants of adsorption $\left(k_{\mathrm{on}}\right)$ and desorption $\left(k_{\mathrm{off}}\right)$ were determined applying a model based on the scaled particle theory (see chapter 3.4.3, page 71) using the relation between the rate constant and the dissociation constant $K_{D}$ (eq. 4.4).

The adsorption rate constant were fitted to the data based on the SPT model (eq. 3.19 and eq. 3.20) that takes mass transport effects $\left(k_{\mathrm{tr}}\right)$ into account. Exemplary time courses of the frequency shift and relative reflectivity change along with the fitting results are depicted in Fig. 4.20 A and B. 

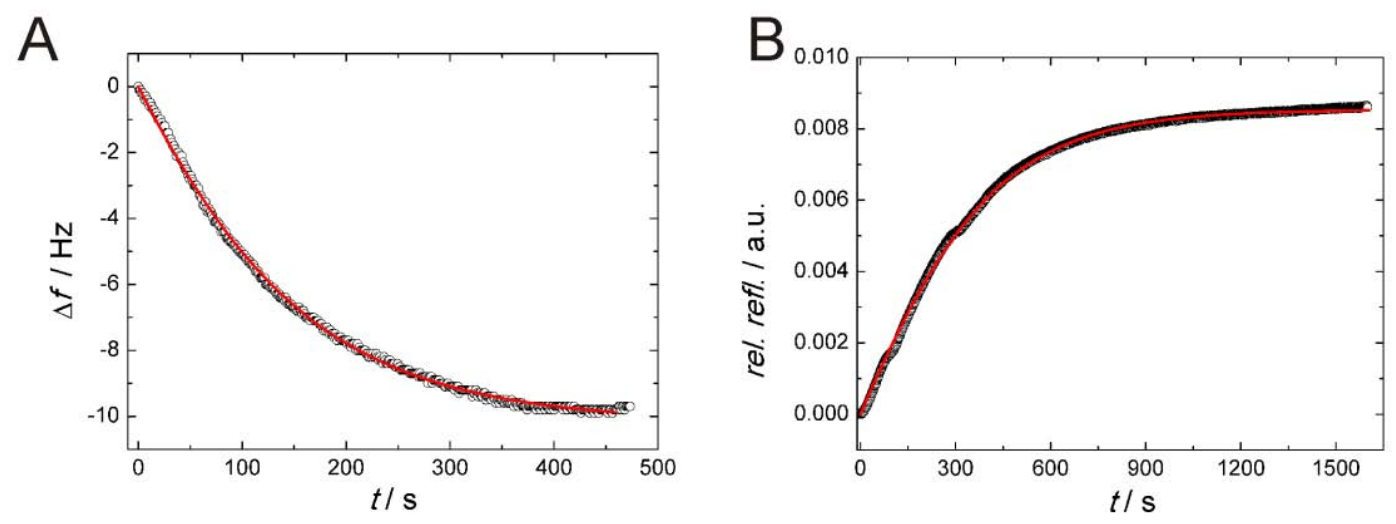

Fig. 4.20 (A) Time dependent frequency shift after the addition of $0.22 \mu \mathrm{M} \mathrm{cPC} 2$ to a DOPC/DOGS-NTA$\mathrm{Ni}$, 9:1 membrane covered with cPC1 in $\mathrm{Ca}^{2+}$ containing buffer $(150 \mathrm{mM} \mathrm{NaCl}, 20 \mathrm{mM}$ HEPES, $1 \mathrm{mM}$ $\mathrm{CaCl}_{2}, \mathrm{pH} 7.4$ ). The rate constant of protein adsorption ( $\left.k_{\text {on }}\right)$ was fitted to the data according to a SPT based model that takes mass transport effects into account $\left(k_{\mathrm{tr}}\right)$. The rate constants were determined to be $k_{\mathrm{on}}=(2.1 \pm 0.5) \cdot 10^{4} \mathrm{M}^{-1} \cdot \mathrm{s}^{-1}, k_{\text {off }}=(1.7 \pm 0.4) \cdot 10^{-3} \mathrm{~s}^{-1}$ and $k_{\mathrm{tr}}=(2.7 \pm 1.3) \cdot 10^{4} \mathrm{M}^{-1} \cdot \mathrm{s}^{-1}$. (B) Time dependent relative reflectivity change after the addition of $0.24 \mu \mathrm{M}$ cPC2 to a DOPC/DOGS-NTA-Ni, 9:1 membrane covered with CPC1 in Ca ${ }^{2+}$-buffer. Rate constants were determined to $k_{\text {on }}=(1.0 \pm 0.4) \cdot 10^{4} \mathrm{M}^{-1} \mathrm{~s}^{-1}, k_{\text {off }}=(0.8 \pm 0.3) \cdot 10^{-}$ ${ }^{3} \mathrm{~s}^{-1}$ and $k_{\mathrm{tr}}=(1.6 \pm 1.1) \cdot 10^{4} \mathrm{M}^{-1} \cdot \mathrm{s}^{-1}$.

The rate constants of adsorption and desorption determined from either QCM or SPR experiments are listed together with the respective dissociation constants in Table 4.2.

Table 4.2 Rate constants $k_{\text {on }}$ and $k_{\text {off }}$ obtained from the changes in $\Delta f$ or relative reflectivity as a function of time measured by the QCM or SPR technique. Fits to the data based on a SPT model were performed. The dissociation constant $K_{\mathrm{D}}$ was determined fitting the parameters of a Langmuir adsorption isotherm to the changes in $\Delta f$ or relative reflectivity as a function of $\mathrm{CPC} 2$ concentration.

\begin{tabular}{c|c|c|c}
$\mathbf{c P C} 2 / \mathbf{c P C} \mathbf{1}$ & $\boldsymbol{k}_{\mathrm{on}} / \mathbf{1 0}^{\mathbf{4}} \mathbf{M}^{-\mathbf{1} \cdot \mathbf{s}^{-\mathbf{1}}}$ & $\boldsymbol{k}_{\text {off }} / \mathbf{1 0}^{-\mathbf{3}} \mathbf{s}^{-\mathbf{1}}$ & $K_{\mathrm{D}} / \mathbf{~} \mathbf{M}$ \\
\hline $\mathrm{QCM}$ & $(2.1 \pm 0.5)$ & $(1.7 \pm 0.4)$ & $(66 \pm 28)$ \\
\hline $\mathrm{SPR}$ & $(1.0 \pm 0.4)$ & $(0.8 \pm 0.3)$ & $(92 \pm 53)$
\end{tabular}


The results demonstrate that the kinetic constants determined from both QCM and SPR data in the same range but not identical within the experimental error.

\subsubsection{Influence of Mass Transport on the Kinetics of the cPC2/cPC1 Complex Formation}

In order to elucidate, whether the mass transport could be minimized, further experiments were performed.

First, the dependence of the concentration dependent rate constant $k_{\mathrm{s}}$ and the mass transport rate constant $k_{\mathrm{tr}}$ on the flow rate was measured by means of QCM experiments. Rate constants were measured as a function of flow rate (Fig. $4.21 \mathrm{~A}$ and B), after adding $0.3 \mu \mathrm{M}$ cPC2 to a DOPC/DOGS-NTA-Ni, 9:1 membrane that was completely covered with cPC1.
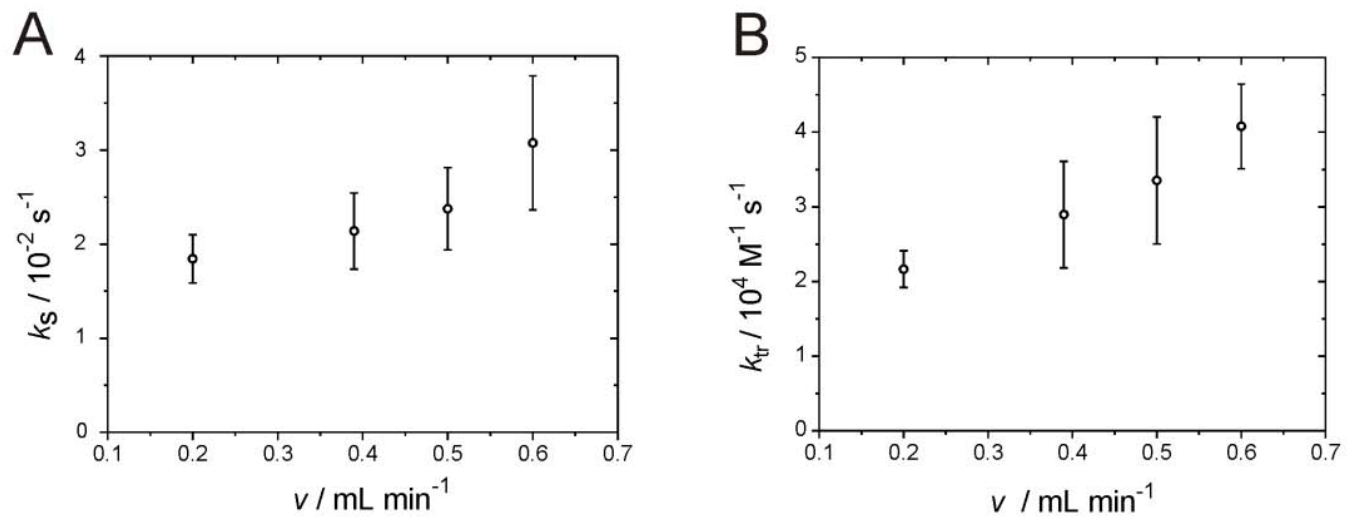

Fig. 4.21 (A) Concentration dependent rate constant $k_{\mathrm{s}}$ and mass transport rate constant $(\mathrm{B})$ as a function of flow rate $(v)$ measured after the addition of $0.3 \mathrm{mM} \mathrm{cPC} 2$ to $\mathrm{CPC} 1$ immobilized on a membrane that was composed of DOPC/DOGS-NTA-Ni, 9:1.

It turned out that, indeed both the transport and hence the CPC2 binding kinetics, could be accelerated by increasing the flow rate from $0.2 \mathrm{ml} / \mathrm{min}$ to $0.6 \mathrm{ml} / \mathrm{min}$, indicated by an increase of both rate constants (in all other experiments, the flow rate was set to 
$0.4 \mathrm{ml} / \mathrm{min}$ ). That no saturation of the values was observed at higher flow rates, that the meass transport influence could be further reduces by increasing the flow rate. However, it was not possible to do so, as the signal to noise ratio of the resonance frequency signal $(\Delta f)$ became unacceptably low with flow rates higher than $0.6 \mathrm{ml} / \mathrm{min}$. The exact values of the measured kinetic rate constants and flow rates are listed in Table 4.3.

Table 4.3 Concentration dependent rate constant $k_{\mathrm{s}}$ and mass transport rate constant $k_{\mathrm{tr}}$ determined at different flow rates.

\begin{tabular}{c|c|c} 
flow rate $/ \mathbf{~} \mathbf{l} \cdot \mathbf{m i n}^{-\mathbf{1}}$ & $\boldsymbol{k}_{\mathrm{s}} / \mathbf{1 0}^{\mathbf{- 2}} \mathbf{s}^{\mathbf{- 1}}$ & $\boldsymbol{k}_{\mathrm{tr}} / \mathbf{1 0}^{\mathbf{4}} \mathbf{M}^{\mathbf{- 1}} \cdot \mathbf{s}^{-\mathbf{1}}$ \\
\hline 0.2 & $(1.8 \pm 0.3), \mathrm{n}=3$ & $(2.2 \pm 0.2)$ \\
\hline 0.4 & $(2.1 \pm 0.4), \mathrm{n}=3$ & $(2.9 \pm 0.7)$ \\
\hline 0.5 & $(2.4 \pm 0.4), \mathrm{n}=4$ & $(3.4 \pm 0.9)$ \\
\hline 0.6 & $(3.1 \pm 0.7), \mathrm{n}=6$ & $(4.1 \pm 0.6)$
\end{tabular}

Besides this, the impact of the CPC1 surface coverage on the mass transport was investigated (flow rate $=0.4 \mathrm{ml} / \mathrm{min}$ ). As reported in the literature, a lower surface concentration of the immobilized binding partner should reduce mass transport effects. ${ }^{19}$ In Fig. 4.22 , the concentration dependent rate constant $k_{\mathrm{s}}$ is plotted against the DOGS-NTA-Ni membrane content. 


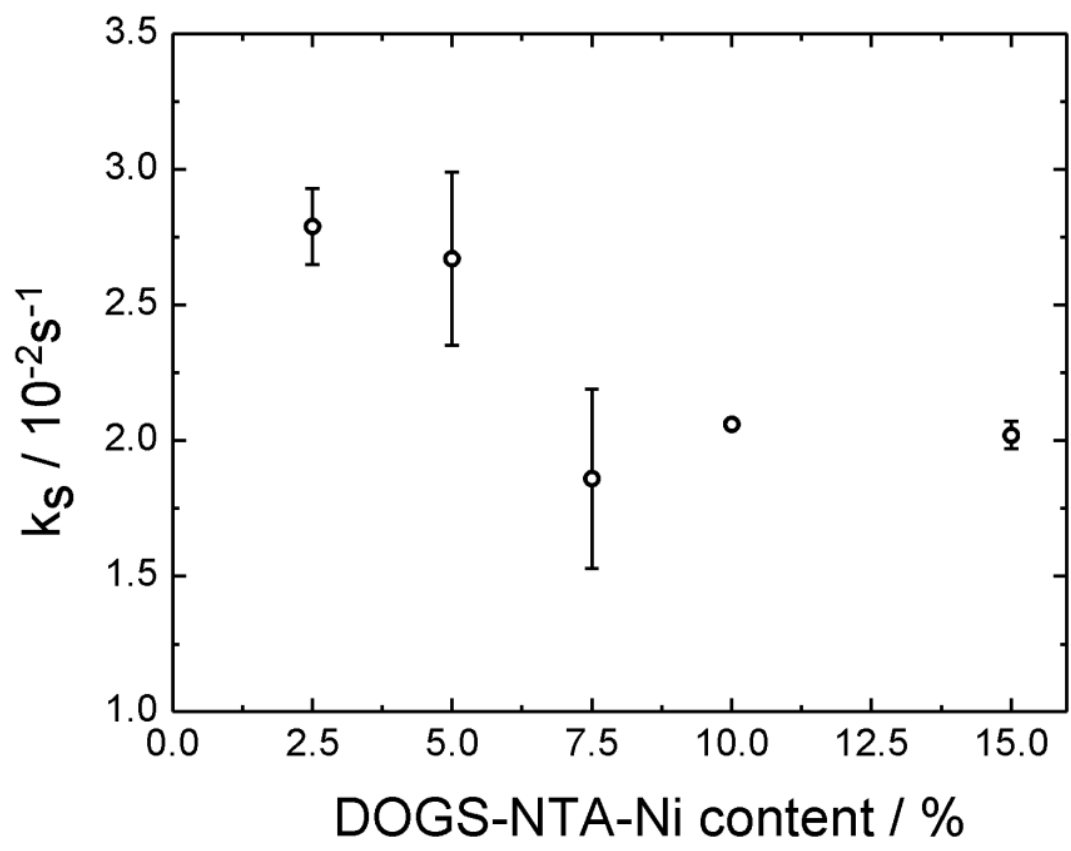

Fig. 4.22 Concentration dependent rate constant as a function of the DOGS-NTA-Ni content of a DOPC solid supported hybrid membrane.

Indeed, $k_{\mathrm{s}}$ is slightly increased at lower DOGS-NTA-Ni contents (2.5 and $5 \mathrm{~mol} \%$ ), which accounts for a reduced mass transport influence. The exact values of $k_{\mathrm{s}}$ are listed together with the respective DOGS-NTA-Ni content and frequency equilibrium shift $\Delta f_{\mathrm{e}}$ in Table 4.4 . 
Table 4.4 Concentration dependent rate constants $k_{\mathrm{s}}$ together with the corresponding DOGS-NTA-Ni contents, and frequency equilibrium shifts $\Delta f_{\mathrm{e}}$.

\begin{tabular}{c|c|c} 
DOGS-NTA-Ni content $/ \%$ & $-\Delta f_{\mathrm{e}} / \mathbf{H z}$ & $\boldsymbol{k}_{\mathrm{s}} / \mathbf{1 0 ^ { - 2 }} \mathbf{s}^{-\mathbf{1}}$ \\
\hline 2.5 & $(8 \pm 3)$ & $(2.3 \pm 0.4), \mathrm{n}=2$ \\
\hline 5 & $(10 \pm 5)$ & $(2.7 \pm 0.3), \mathrm{n}=3$ \\
\hline 7.5 & $(12 \pm 1)$ & $(1.9 \pm 0.2), \mathrm{n}=2$ \\
\hline 10 & $(13)$ & $2.0, \mathrm{n}=1$ \\
\hline 15 & $(16 \pm 1)$ & $(2.0 \pm 0.1), \mathrm{n}=2$
\end{tabular}

\subsection{Discussion}

The aim of this work was to establish a biosensor screening assay based on solid supported membranes that allows to quantitatively investigate the interaction between membrane coupled protein $\mathrm{CPC} 2$ and its putative interaction partners, starting with cPC1. The hexahistidine tagged ligand protein $\mathrm{CPC} 1$ was therefore specifically immobilized on a DOPC membrane surface containing $10 \mathrm{~mol} \%$ of the DOGS-NTA-Ni receptor lipid until surface saturation was reached. The analyte protein CPC2 was the given to the system and binding was monitored as either a change in resonance frequency $\Delta f$ or relative reflectivity in a time resolved and label-free manner.

In order to develop this membrane based assay, first the quality of the solid supported hybrid membrane was controlled and the existence of large membrane defects was ruled out. For membranes prepared on the gold electrode of an AT-cut quartz crystal used for QCM experiments the determined specific capacitance for the octanethiol monolayer $C_{\text {от }}=(2.3 \pm 0.2) \mu \mathrm{F} / \mathrm{cm}^{2}(\mathrm{n}=27)$, the hybrid bilayer $C_{\mathrm{BL}}=(1.1 \pm 0.1) \mu \mathrm{F} / \mathrm{cm}^{2}$ $(\mathrm{n}=26)$ and calculated phospholipid monolayer $C_{\mathrm{ML}}=(2.1 \pm 0.5) \mu \mathrm{F} / \mathrm{cm}^{2}(\mathrm{n}=26)$ were in 
accordance with results reported in literature. Subramanian et al. determined a final capacitance of $2.45 \mu \mathrm{F} / \mathrm{cm}^{2}$ for an octanethiol monolayer on gold ${ }^{26}$, while Herrig et al. reported a capacitance of Сот $=(2.0 \pm 0.2) \mu \mathrm{F} / \mathrm{cm}^{2}$ for the octanethiol monolayer and $C_{\mathrm{BL}}=(1.1 \pm 0.2) \mu \mathrm{F} / \mathrm{cm}^{2}$ for a hybrid bilayer system composed of POPC with variable $\mathrm{PIP}_{2}$ content. ${ }^{15}$ Independent studies performed by Steinem et al. and Plant et al. found a phospholipid monolayer capacitance of $C_{M L}=(1.96 \pm 0.23) \mu F / \mathrm{cm}^{2}$ and $C_{\mathrm{ML}}=1.92 \mu \mathrm{F} / \mathrm{cm}^{2}$ in case of POPC monolayers. This value is in agreement with the results obtained for monolayers composed of DOPC/DOGS-NTA-Ni, 9:1. In this study, analyzing in situ spreading experiments performed by the SPR technique showed that the resulted monolayer thickness as well as the spreading kinetics were dependent on both the lipid composition and the ionic strength of the buffer. While in low salt E1 buffer and $10 \mathrm{mM} \mathrm{CaCl}_{2}(50 \mathrm{mM} \mathrm{KCl}, 20 \mathrm{mM}$ TRIS/HCl, $0.1 \mathrm{mM}$ EDTA, $0.1 \mathrm{mM} \mathrm{NaN}$, $\mathrm{pH}$ 7.4) the monolayer thickness of a DOPC/DOGS-NTA-Ni, 9:1 membrane was only $d=(1.2 \pm 0.5) \mathrm{nm}(\mathrm{n}=3)$, a layer thickness of $d=(3.4 \pm 1.6) \mathrm{nm}(\mathrm{n}=17)$ resulted, if vesicles of the same composition were spread in high salt P1 buffer (500 mM NaCl, $20 \mathrm{mM}$ TRIS/HCl, pH 8.0). Pure DOPC monolayers were formed with a final thickness of $d=3.9 \mathrm{~nm}(\mathrm{n}=1)$ and a kinetic that indicates the adhesion of vesicles on the membranes surface monitored as a steady increase of the relative reflectance (see Fig. 4.10). In previous studies by Naumean et al. and Rossi et al. a monolayer thickness of $d=2 \mathrm{~nm}$ and $d=(2.2 \pm 0.3) \mathrm{nm}$ for egg-PC was determined by means of SPR experiments. ${ }^{27,28}$ Both the thickness value of the pure DOPC membrane and membranes containing DOPC/DOGS-NTA-Ni, 9:1 spread in high salt P1 buffer were larger than the values reported in literature. Possible reasons for this discrepancy are the formation of multilayers or the adhesion of vesicles on the membrane surface. It is well known that vesicles tend to adhere to surfaces or to each other as a function of their $\xi$-potential, a value that depends on the lipid headgroup properties and the ionic strength of the surrounding solution..$^{29,30}$ In 2010 Jothi et al. showed that DOPC vesicles tend to form multilayers if the $\mathrm{NaCl}$ concentration was increased from 5 to $20 \mathrm{mM} \cdot{ }^{31}$ They concluded that high $\mathrm{NaCl}$ concentration promote the coalescence of vesicles, which results in the formation 
of multilayers. They also found that an increased amount of unruptured vesicles adhered on the membrane surface at high electrolyte concentrations. The corresponding spreading curves measured by means of QCM or reflectrometry showed the same course as the DOPC spreading curve in this work. As the monolayer thickness of DOPC/DOGS-NTA-Ni, 9:1 membranes formed in low salt E1 buffer with addition of $10 \mathrm{mM} \mathrm{CaCl}_{2}$ was slightly lower than average values reported in the, one could assume that this was caused by membrane defects. The hexahistidine tagged protein binding experiment however, showed that specific binding of ezrin to a DOPC membrane with a $10 \mathrm{~mol} \%$ DOGS-NTA-Ni content and further elution with $250 \mathrm{~mm}$ imidazol containing buffer took place. By means of this SPR experiment, it was demonstrated that no unspecific binding in potential membrane defects occurred, as all ezrin could be eluted after rinsing with the imidazol containing buffer (see Fig. 4.15 B).

Besides this, the specificity of hexahistidine tagged protein binding to the DOGS-NTANi receptor lipid was demonstrated by the only marginal, unspecific binding of the protein ezrin and PIGEA14 to pure DOPC membranes measured by means QCM and SPR experiments (Fig. 4.14 and Fig. 4.15). To more explicitly examine the DOGS-NTANi-hexahistindine tagged protein interaction in terms of a possible DOGS-NTA recruitment, QCM experiments were performed in order to investigate the frequency response after the addition of $0.3 \mu \mathrm{M}$ PIGEA14 to membranes with various DOGS-NTANi contents (0-15 mol\%, see Fig. 4.14 B). Mokhtarzada et al. determined the radius of PIGEA14 to be $3 \mathrm{~nm}$ by means of dynamic light scattering, a value relatively large for a protein with a mass of only $14 \mathrm{kDa} .{ }^{32}$ Hypothetically assuming that value, a DOGSNTA-Ni content of $2.5 \mathrm{~mol} \%$ would be sufficient to obtain a maximal surface coverage $\left(\right.$ A PIgea14 $=28.28 \mathrm{~nm}^{2}$, AdOGs-NTA-Ni $\left.=0.7 \mathrm{~nm}^{2}\right)$. However, as summarized in Table 3.1 the DOGS-NTA-Ni lipid offers only two potential binding sites and a protein with six accessible histidine residues would most likely bind with a stoichiometry of 1-3. QCM experiments suggested that maximal coverage of PIGEA14 was achieved at $\sim 7.5 \mathrm{~mol} \%$ DOGS-NTA-Ni. This implies the recruitment of three DOGS-NTA-Ni molecules under one PIGEA14 molecule. Although it makes sense that more than one receptor lipid 
binds a hexahistidine tagged protein a radius of $3 \mathrm{~nm}$ for a protein with a mass of $14 \mathrm{kDa}$ appears to be quite large. Mokhtarzada et al. augmented than this high value could be caused by protein dimerization, partial unfolding or formation of elongated coiled coil structures. ${ }^{32}$ Thus, if no ideal, globular geometry of PIGEA14 can be expected or the radius of PIGEA14 may be over determined the binding stoichiometry of PIGEA14/DOGS-NTA-Ni would be less than 1:3.

To test whether the investigation of protein-protein interaction by means of the newly developed membrane based biosensor assay was possible, the hexahistidine tagged CPolycystin-1 (cPC1) and its specific interactor C-Polycystin-2 (cPC2) were used. Owing to the fact that the dissociation constants determined by fitting the parameters of a Langmuir adsorption isotherm (see Fig. 4.17 and eq. 4.1) and the plot and linear fit of the concentration dependent rate constant $k_{\mathrm{s}}$ against the $\mathrm{cPC} 2$ concentration (see Fig. 4.19 and eq. 4.4) did not match, it was concluded that the simple Langmuir model was not sufficient to fully describe the adsorption phenomenon of proteins on solid interfaces. Difference in the thermodynamically and kinetically determined dissociation constants normally occur due to mass transport effect during the binding process. There are several possibilities to minimize the influence of mass transport effects on a binding study. ${ }^{19}$ First, a high flow rate would reduce the height of the unstirred layer above the sensor surface. Second, lowering the content of ligand protein immobilized on the sensor surface would lower the depletion rate and thus decrease the impact of the mass transport on the binding kinetics. Testing these two options by means of the QCM technique, we found that increasing the flow rate indeed led to a reduction of mass transport effects, indicated by higher values of $k_{\mathrm{s}}$ and $k_{\text {tr.. }}$ Unfortunately, a lower signal to noise ratio of the resonance frequency signal, forced us to work with a flow rate $(0.6 \mathrm{ml} / \mathrm{min})$, at which mass transport still seems to influence the reaction kinetics; as there was no saturation in the course of the measured values (see Fig. 4.21 A and B).

Lowering the surface capacity of the ligand protein indeed leads to a reduction of mass transport effects, as higher adsorption rate constants were determined for DOGS-NTANi contents of 2.5 and $5 \mathrm{~mol} \%$ (Fig. 4.22). Nevertheless, it also causes a twofold de- 
crease of the measured frequency shift $\Delta f_{\mathrm{e}}$, which resulted in higher errors of the meas-

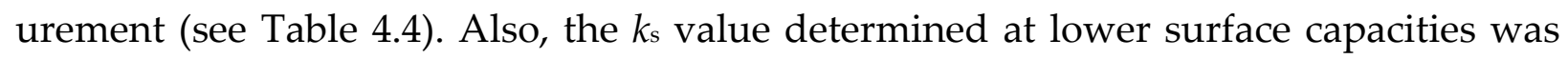
only in the range of the one measure at a flow rate of $0.6 \mathrm{ml} / \mathrm{min}$. Thus, it can be strongly assumed that mass transport was still influencing the kinetics. Combining the effects of an increased flow rate $(0.6 \mathrm{ml} / \mathrm{min})$ and a low cPC1 surface capacity (DOGS-NTA-Ni content $=5 \%$ ) would probably give the best result possibly in terms of minimizing mass transport effects, but would also cause an even worse signal to noise ratio.

Different methods and models that deal with a non ideal adsorption behavior of biomolecules on solid interfaces are described and discussed in chapter 1 and 9. Here, a model that bases on the scaled particle theory (SPT) was used (see chapter 3.4.3) to determine the rate constants of adsorption $\left(k_{\mathrm{on}}\right)$, desorption $\left(k_{\mathrm{off}}\right)$ and the transport of proteins to the biosensor surface $\left(k_{\mathrm{tr}}\right)$. The determined rate constant of mass transport was in good agreement with values found in the literature..$^{33,34}$

Regarding the $\mathrm{CPC} 2 / \mathrm{CPC} 1$ binding kinetics, it turned out that astonishingly, while the adsorption and desorption kinetics of cPC2 exhibit a reversibility of $(66 \pm 13) \%, n=5$ being examined by means of the QCM, only a reversibility of $(36 \pm 16) \%, n=7$ was monitored by means of the SPR technique (Fig. 4.16 A and B). This surprising finding consequently had an influence on the kinetic rate constants of adsorption and desorption determined by the QCM and SPR technique respectively. Both the rate of adsorption and desorption determined by SPR experiments were twofold smaller compared to the ones obtained by QCM experiments, while the dissociation constants did not significantly differ (Table 4.2). The most obvious explanation is that secondary binding/desorption processes took place during the SPR experiments. This could be due to binding of CPC2 to the hydrophobic surface of putative membrane defects or to denatured cPC1 inside the defects. Both binding events would explain the non reversible content of the desorption as denatured cPC2 would not as easily desorb as specifically bound protein. Nevertheless, the elution experiment performed with ezrin (see Fig. 4.15) excludes this assumption. Protein denaturation might also have been caused by the long residence time of the laser spot. Other effects such as a putative rebinding of 
CPC2 during the desorption process caused by the different flow cell geometry of the SPR experiment compared to the QCM setup, are also unlikely, because they would only reduce the rate of desorption, but not explain the lower reversibility. As the lower reversibility of the $\mathrm{CPC} / \mathrm{cPC} 1$ binding during SPR experiments could neither be explained nor prevented, the QCM technique was chosen for all studies that were performed to quantitatively investigate the binding of protein-protein interactions (see chapter 5 and 6).

\subsection{Co-workers}

Schön, Markus; Schatz, Michaela; Hoffmeister, Hellen; Witzgall, Ralph; Janshoff, Andreas 


\subsection{References}

[1] Celić, A., Petri, E. T., Demeler, B., Ehrlich, B. E., and Boggon, T. J. (2008) Domain mapping of the polycystin-2 C-terminal tail using de novo molecular modeling and biophysical analysis. J. Biol. Chem. 283, 28305-28312.

[2] The International Polycystic Kidney Disease Consortium* (1995) Polycystic kidney disease: the complete structure of the PKD1 gene and its protein. Cell 81, 289-298.

[3] Hanaoka, K., Qian, F., Boletta, A., Bhunia, A. K., Piontek, K., Tsiokas, L., Sukhatme, V. P., Guggino, W. B., and Germino, G. G. (2000) Co-assembly of polycystin-1 and -2 produces unique cation-permeable currents. Nature 408, 990-994.

[4] Nauli, S. M., Alenghat, F. J., Luo, Y., Williams, E., Vassilev, P., Li, X., Elia, A. E. H., Lu, W., Brown, E. M., Quinn, S. J., Ingber, D. E., and Zhou, J. (2003) Polycystins 1 and 2 mediate mechanosensation in the primary cilium of kidney cells. Nat. Genet. 33, 129-137.

[5] Cooper, M. A. (2003) Label-free screening of bio-molecular interactions. Analytical and Bioanalytical Chemistry 377, 834-842.

[6] Haake, H.-M., Schütz, A., and Gauglitz, G. (2000) Label-free detection of biomolecular interaction by optical sensors. Fresen. J. Anal. Chem. 366, 576-585.

[7] Rich, R. L., and Myszka, D. G. (2008) Survey of the year 2007 commercial optical biosensor literature. J. Mol. Recognit. 21, 355-400.

[8] Cheng, C. I., Chang, Y.-P., and Chu, Y.-H. (2012) Biomolecular interactions and tools for their recognition: focus on the quartz crystal microbalance and its diverse surface chemistries and applications. Chem Soc Rev 41, 1947-1971. 
[9] Janshoff, Galla, and Steinem (2000) Piezoelectric Mass-Sensing Devices as Biosensors-An Alternative to Optical Biosensors? Angew. Chem. Int. Ed. Engl 39, 4004-4032.

[10] Tinazli, A., Tang, J., Valiokas, R., Picuric, S., Lata, S., Piehler, J., Liedberg, B., and Tampé, R. (2005) High-Affinity Chelator Thiols for Switchable and Oriented Im mobilization of Histidine-Tagged Proteins: A Generic Platform for Protein Chip Technologies. Chem. Eur. J. 11, 5249-5259.

[11] Fong, C.-C., Wong, M.-S., Fong, W.-F., and Yang, M. (2002) Effect of hydrogel matrix on binding kinetics of protein-protein interactions on sensor surface. Analytica Chimica Acta 456, 201-208.

[12] Castellana, E. T., and Cremer, P. S. (2007) Solid-Supported Lipid Bilayers: From Biophysical Studies to Sensor Design. ChemInform 38.

[13] Janshoff, A., Steinem, C., Sieber, M., and Galla, H.-J. (1996) Specific binding of peanut agglutinin to GM1 -doped solid supported lipid bilayers investigated by shear wave resonator measurements. Eur. Biophys. J. 25, 105-113.

[14] Kastl, K., Ross, M., Gerke, V., and Steinem, C. (2002) Kinetics and thermodynamics of annexin A1 binding to solid-supported membranes: a QCM study. Bio chemistry 41, 10087-10094.

[15] Herrig, A., Janke, M., Austermann, J., Gerke, V., Janshoff, A., and Steinem, C. (2006) Cooperative Adsorption of Ezrin on PIP 2 -Containing Membranes. Biochemistry 45, 13025-13034.

[16] Rabe, M., Verdes, D., and Seeger, S. (2011) Understanding protein adsorption phenomena at solid surfaces. Adv Colloid Interface Sci 162, 87-106.

[17] Feder, J. (1980) Random sequential adsorption. J. Theor. Biol. 87, 237-254.

[18] Brusatori, and Van Tassel PR (1999) A Kinetic Model of Protein Adsorption/Surface-Induced Transition Kinetics Evaluated by the Scaled Particle Theory. J. Colloid. Interf. Sci. 219, 333-338. 
[19] Myszka, D. G. (1997) Kinetic analysis of macromolecular interactions using surface plasmon resonance biosensors. Curr. Opin. Biotechnol. 8, 50-57.

[20] Schuck, P., and Minton, A. P. (1996) Analysis of Mass Transport-Limited Binding Kinetics in Evanescent Wave Biosensors. Analytical Biochemistry 240, 262-272.

[21] Chatelier, R., and Minton, A. (1996) Adsorption of globular proteins on locally planar surfaces: models for the effect of excluded surface area and aggregation of adsorbed protein on adsorption equilibria. Biophys. J. 71, 2367-2374.

[22] Behn, D., Bosk, S., Hoffmeister, H., Janshoff, A., Witzgall, R., and Steinem, C. (2010) Quantifying the interaction of the C-terminal regions of polycystin-2 and polycystin-1 attached to a lipid bilayer by means of QCM. Biophys. Chem. 150, 47-53.

[23] Pan, W. D. C. J. T. N. J. (1996) Kinetics of Alkanethiol Adsorption on Gold. Langmuir 12, 4469-4473.

[24] Abbott, N. L., Gorman, C. B., and Whitesides, G. M. (1995) Active Control of Wetting Using Applied Electrical Potentials and Self- Assembled Monolayers. Langmuir 11, 16-18.

[25] Bosk, S., Braunger, J. A., Gerke, V., and Steinem, C. (2011) Activation of F-actin binding capacity of ezrin: synergism of $\mathrm{PIP}_{2}$ interaction and phosphorylation. Biophys. J. 100, 1708-1717.

[26] Subramanian, R., and Lakshminarayanan, V. (2000) A study of kinetics of ad sorption of alkanethiols on gold using electrochemical impedance spectroscopy. Electrochim. Acta 45, 4501-4509.

[27] Naumann, R., Jonczyk, A., Kopp, R., van Esch, J., Ringsdorf, H., Knoll, W., and Gräber, P. (1995) Incorporation of Membrane Proteins in Solid-Supported Lipid Layers. Angew. Chem. Int. Ed. Engl 34, 2056-2058.

[28] Rossi, C., Homand, J., Bauche, C., Hamdi, H., Ladant, D., and Chopineau, J. (2003) Differential mechanisms for calcium-dependent protein/membrane asso- 
ciation as evidenced from SPR-binding studies on supported biomimetic membranes. Biochemistry 42, 15273-15283.

[29] Egawa, H., and Furusawa, K. (1999) Liposome Adhesion on Mica Surface Studied by Atomic Force Microscopy. Langmuir 15, 1660-1666.

[30] Lis, L. J., McAlister, M., Fuller, N., Rand, R. P., and Parsegian, V. A. (1982) Interactions between neutral phospholipid bilayer membranes. Biophys. J. 37, 657665.

[31] Edward Gnana Jothi, G., Kamatchi, S., and Dhathathreyan, A. (2010) Adsorption of DOPC vesicles on hydrophobic substrates in the presence of electrolytes: A QCM and reflectometry study. J. Chem. Sci. 122, 341-348.

[32] Mokhtarzada, S., Yu, C., Brickenden, A., and Choy, W.-Y. (2011) Structural characterization of partially disordered human Chibby: insights into its function in the Wnt-signaling pathway. Biochemistry 50, 715-726.

[33] Mol, N. J. de, Plomp, E., Fischer, M. J., and Ruijtenbeek, R. (2000) Kinetic Analysis of the Mass Transport Limited Interaction between the Tyrosine Kinase lck SH2 Domain and a Phosphorylated Peptide Studied by a New Cuvette-Based Surface Plasmon Resonance Instrument. Analytical Biochemistry 279, 61-70.

[34] Myszka, D. G., Morton, T. A., Doyle, M. L., and Chaiken, I. M. (1997) Kinetic analysis of a protein antigen-antibody interaction limited by mass transport on an optical biosensor. Biophys. Chem. 64, 127-137. 



\section{Quantifying the Interaction of the $C$ -}

terminal Regions of Polycystin-2 and Polycystin-1 Attached to a Lipid Bilayer by Means of QCM

\subsection{Abstract}

The $p k d 1$ and $p k d 2$ genes encode for the proteins polycystin-1 (PC1) and polycystin-2 (PC2). These genes are mutated in patients diagnosed with autosomal dominant polycystic kidney disease. PC1 and PC2 interact via their C-terminal, cytosolic regions, which is an essential step in the regulation of cell proliferation and differentiation.

Here, we applied an earlier developed assay that allowed us to quantitatively monitor the interaction of the C-terminal region of PC1 (cPC1) with that of PC2 (cPC2) to be able to answer the question of how $\mathrm{Ca}^{2+}$ influences the PC1/PC2 complex formation. By means of the quartz crystal microbalance (QCM) technique, we were able to determine binding affinities and kinetic constants of the $\mathrm{cPC} 1 / \mathrm{cPC} 2$ interaction using a model based on the scaled particle theory. The results suggest that CPC2 forms trimers in solution in the absence of $\mathrm{Ca}^{2+}$, which bind in a one step process to $\mathrm{CPC} 1$. 


\subsection{Introduction}

The autosomal dominant polycystic kidney disease (ADPKD) is one of the most common monogenetic disorders that results in renal cysts development and finally leads to end stage renal disease and renal failure. ${ }^{1}$ ADPKD occurs in 1:1000 humans as a result of a mutation in one of two genes, $p k d 1$ or $p k d 2$. In $85 \%$ of the individuals diagnosed with ADPKD $p k d 1$ is mutated, while in $5-15 \%$ of the cases it is $p k d 2 .^{2} p k d 1$ and $p k d 2$ encode for the proteins polycystin-1 (PC1) and polycystin-2 (PC2). PC1 is a $462 \mathrm{kDa}$ integral membrane protein composed of 11 transmembrane-spanning regions, with a large extracellular N-terminus, and a smaller intracellular C-terminal part.

As PC1 is located in the plasma membrane, its extracellular N-terminal domain is capable of acting as a sensor unit for fluid shear stress and it is thus very likely that PC1 plays a role in cell proliferation and differentiation.,4 PC2 is composed of 968 amino acids $(\sim 110 \mathrm{kDa})$ with both the $C$ - and $N$-terminus located inside the cell. With six membrane spanning regions, PC2 can be classified as a member of the transient receptor potential (TRP) channels (TRPP2). ${ }^{5}$ Its C-terminus harbors an EF-hand motif (Ala ${ }^{753}$ $\mathrm{Asp}^{781}$ ) as a potential $\mathrm{Ca}^{2+}$ binding site as well as a retention signal for the endoplasmic reticulum (ER) $\left(\mathrm{Asp}^{790}-\mathrm{Ser}^{820}\right){ }^{6}$

Although it has been shown that PC2 is located in the plasma membrane in certain cell lines, it is more commonly found at the ER acting as a $\mathrm{Ca}^{2+}$ permeable, non-selective cation channel.7,8 Both PC1 and PC2 are supposed to have a C-terminal coiled-coil domain, which are discussed to mediate the formation of a heteromeric protein-protein complex. 9,10

As PC2 and PC1 are only colocalized in the plasma membrane of certain cell lines, i.e. the primary cilium of kidney cells, the physiological role of the heteromeric proteinprotein complex formation has been discussed intensively. The primary cilium could act as an antenna and gets bent due to fluid movement in the extracellular space as suggested by Nauli et al.. ${ }^{4}$ This mechanical stress is sensed by PC1, which transmits the 
signal from the extracellular space to the cytosol by forming a protein-protein complex with PC2. Owing to the proposed conformational change of $\mathrm{PC} 2$, a $\mathrm{Ca}^{2+}$ influx is induced that activates the intracellular rynodine receptor leading to an increase of intracellular $\mathrm{Ca}^{2+}$ concentration. This signal transduction cascade is presumably responsible for tissue morphology, cell proliferation and differentiation.

Besides this activation caused by fluid shear stress, a regulation mediated by cell surface receptor stimulation as well as cell adhesion activated regulation has been proposed. ${ }^{9}$ By electrophysiological studies on chinese hamster ovary cells containing $p k d 1$ and $p k d 2$ genes, Hanaoka et al. ${ }^{11}$ found that only a co-assembly of PC1 and PC2 produces a cation channel. Cai et al. ${ }^{12}$ discovered that the channel activity depends on the cytoplasmic $\mathrm{Ca}^{2+}$ concentration and can be modulated by $\mathrm{Ser}^{812}$ phosphorylation. In a recent study by Schumann et al. ${ }^{13}$, the role of $\mathrm{Ca}^{2+}$ to induce conformational changes in a C-terminus of PC2 ( $\mathrm{CPC} 2)$ has been elucidated in detail. By means of circular dichroism-, fluorescence-, and NMR spectroscopy, they were able to identify two $\mathrm{Ca}^{2+}$ binding sites with different affinities $\left(K_{\mathrm{D} 1}=55 \mu \mathrm{M}, K_{\mathrm{D} 2}=179 \mu \mathrm{M}\right)$. Moreover, they suggested a $\mathrm{Ca}^{2+}$-dependent homodimerisation of $\mathrm{cPC} 2$. In the absence of $\mathrm{Ca}^{2+}, \mathrm{cPC} 2$ putatively forms a homodimer, while in the presence of $\mathrm{Ca}^{2+}$ it exists as a momoner. Yu et al. ${ }^{14}$ however, reported that $\mathrm{CPC} 2$ forms a trimer, both in solution and crystal structure. Pull down experiments with cPC2 mutants demonstrated that only the full length, trimer forming fragment (Gly $\left.{ }^{833-G l y}{ }^{895}\right)$ is capable of interacting with the C-terminal coiled-coil domain of PC1 (cPC1). ${ }^{14}$ The protein-protein complex was composed of three cPC2 molecules and one cPC1 domain. These findings suggest that $\mathrm{Ca}^{2+}$ is capable of modulating the complex formation of $\mathrm{CPC} 1$ with $\mathrm{cPC} 2$.

To investigate the protein-protein complex formation, we made use of an earlier established assay (see chapter 4) that allows us to quantitatively monitor the interaction of CPC1 with CPC2 in a time resolved and label free manner. The assay should allow us to answer questions of how $\mathrm{Ca}^{2+}$ influences the PC1/PC2 complex formation. The idea was to mimic the natural environment of the $\mathrm{C}$-terminal part of $\mathrm{PC} 1$ by attaching $\mathrm{CPC} 1$ to a lipid bilayer. To achieve this goal, we used solid supported membranes and immobi- 
lized cPC1 via its histidine tag to a $\mathrm{Ni}^{2+}$ nitrilotriacetic acid (NTA-Ni) modified lipid. By means of the quartz crystal microbalance (QCM) technique we were able to investigate the binding of $\mathrm{CPC} 2$ without attaching any label in a time resolved manner. From the obtained results, we managed to extract binding affinities and kinetic parameters applying the scaled particle theory ${ }^{15}$ as a function of the $\mathrm{Ca}^{2+}$-concentration.

The QCM technique has proven to be a powerful tool to observe adsorption processes at solid-liquid interfaces in a label-free and time-resolved manner. ${ }^{16,17}$ Owing to the fact that the frequency response of the quartz crystal is directly proportional to the adsorbed mass at the solid-liquid interface, the QCM techniques allows to study binding processes such as protein-membrane interactions, protein-protein interactions or cell and liposome adhesion. ${ }^{16,18-22}$ Additionally, viscoelastic changes during the binding process can be monitored, which gives a closer insight into the nature of the adhered biomaterial. ${ }^{23,24}$

\subsection{Results}

\subsubsection{Analysis of cPC2 as a Function of $\mathrm{Ca}^{2+}$}

Besides the analysis of secondary structure elements performed by means of circular dichroism (CD) spectroscopy in phosphate buffer (see chapter 3.4.2) described in chapter 6.3.2, CD spectra of $\mathrm{CPC} 2$ were recorded as a function of $\mathrm{Ca}^{2+}$ concentration to find out whether CPC2 undergoes $\mathrm{Ca}^{2+}$ induced structural changes.

Fig. 5.1 A shows the CD spectra of cPC2 (black line) in an EDTA containing buffer (150 mM NaCl, $0.1 \mathrm{mM}$ EDTA, $20 \mathrm{mM}$ TRIS/HCl, pH 7.4). After the addition of $1.4 \mathrm{mM}$ $\mathrm{CaCl}_{2}$ (red line, $\mathrm{Ca}^{2+}$ titration buffer: $150 \mathrm{mM} \mathrm{NaCl}, 20 \mathrm{mM} \mathrm{CaCl}$, $20 \mathrm{mM}$ TRIS/HCl, $\mathrm{pH}$ 7.4) the spectra is shifted to greater negative values of mean residue ellipticity $(\Theta)$ indicating a change of secondary structural elements upon $\mathrm{Ca}^{2+}$ binding to $\mathrm{CPC} 2$ at the 
EF Hand (see Fig. 4.2). This effect was reproducibly obtained also the other way around (addition of EDTA to a $\mathrm{Ca}^{2+}$ containing buffer), which showed the exact opposite response. After each $\mathrm{CaCl}_{2}$ or EDTA addition the solution was mixed and allowed to equilibrate for $10 \mathrm{~min}$ to ensure $\mathrm{Ca}^{2+}$ binding or desorption. Seven averaged spectra were taken from $260-200 \mathrm{~nm}$ at $20^{\circ} \mathrm{C}$ with a scan speed of $20 \mathrm{~nm} / \mathrm{min}$ in each experiment.
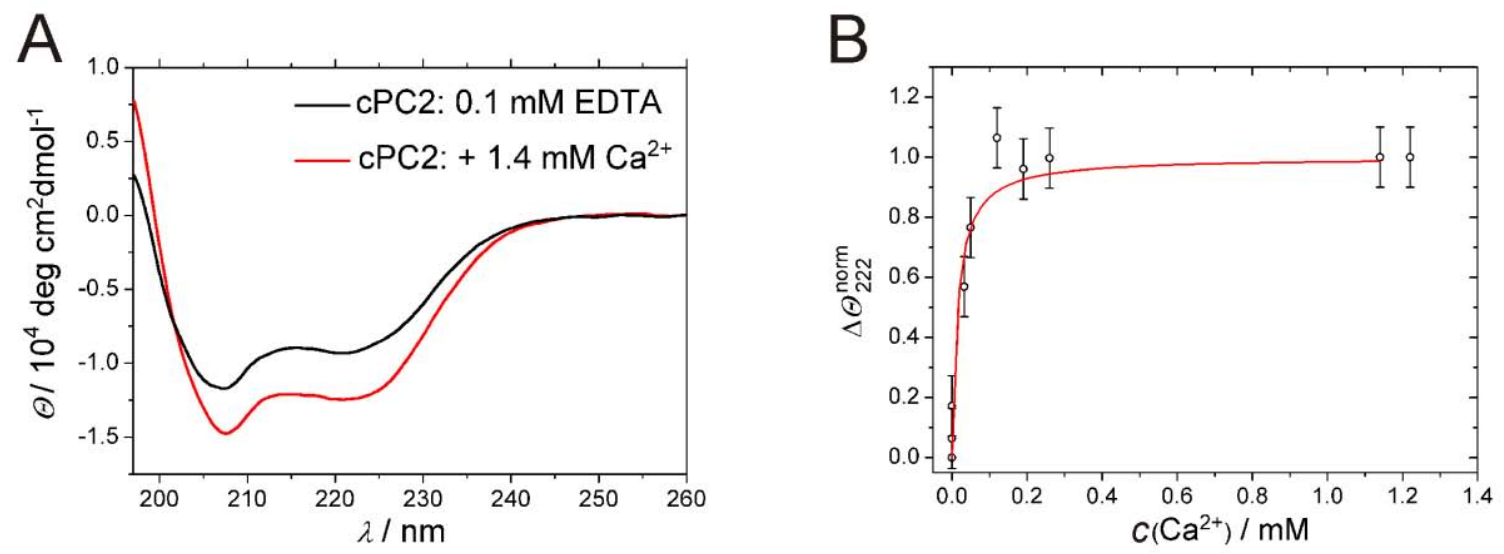

Fig. 5.1 (A) CD spectra of cPC2 recorded in the absence (black line) and after the addition of $\mathrm{Ca}^{2+}$ (red line). (B) cPC2/ $\mathrm{Ca}^{2+}$-titration isotherm that shows the change of normalized mean residue ellipticity $\Delta \Theta_{222}$ after addition of different $\mathrm{Ca}^{2+}$ concentrations. A dissociation constant of $K_{\mathrm{D}}=(15 \pm 4 \mu \mathrm{M})$ was determined assuming a 1:1 binding stoichiometry.

In addition, it was aimed to quantify the $\mathrm{Ca}^{2+} / \mathrm{CPC} 2$ binding by means of a CD-titration experiment. Different $\mathrm{Ca}^{2+}$ concentrations were titrated to a cPC2 solution in EDTA containing buffer. The normalized mean residue ellipticity at $222 \mathrm{~nm} \Delta \Theta_{222}$ was plotted against the free $\mathrm{Ca}^{2+}$ concentration (Fig. $5.1 \mathrm{~B}$ ). The free $\mathrm{Ca}^{2+}$ concentration was calculated using EDTA $\mathrm{Ca}^{2+}$ binding constants by means of a self written MathCad routine.

The parameters of eq. 5.1 were fitted to the obtained data: 


$$
\Delta \theta_{222}^{\text {norm }}=\frac{\left[\mathrm{Ca}^{2+}\right]^{x}}{K_{\mathrm{D}}+\left[\mathrm{Ca}^{2+}\right]^{x}}
$$

with $\Delta \theta_{222}^{\text {norm }}$ as normalized mean residue ellipticity at $222 \mathrm{~nm}, \mathrm{x}$ as the stoichiometry parameter. A dissociation constant of $K_{\mathrm{D}}=(15 \pm 4 \mu \mathrm{M})$ was determined assuming a 1:1 binding stoichiometry.

\subsubsection{Immobilization of cPC1 on a Solid Supported Membrane}

To investigate the interaction of $\mathrm{CPC} 1$ and $\mathrm{cPC} 2$, we first established an immobilization strategy for $\mathrm{CPC} 1$ on the surface of the gold electrode of a quartz plate, which provides a natural environment for $\mathrm{CPC} 1$ mimicking the situation in the living cell including that the lateral mobility of the protein on the surface is guaranteed (see chapter 4.3.4)

Following this idea, we made use of solid supported membranes composed of DOPC doped with $10 \mathrm{~mol} \%$ DOGS-NTA-Ni. The high content of DOGS-NTA-Ni ensures an almost irreversible binding of $\mathrm{CPC} 1$ to the chelator lipid via its histidine tag as well as full coverage of the membrane with $\mathrm{CPC1}$.This strategy is advantageous over a covalent attachment of the protein to the surface, where the protein is completely laterally immobile.

We investigated the success of immobilization by the QCM technique. cPC1 was added to the DOPC/DOGS-NTA-Ni membranes in both $\mathrm{Ca}^{2+}$-free and $\mathrm{Ca}^{2+}$-containing buffer with increasing concentrations and the change in resonance frequency of the $5 \mathrm{MHz}$ quartz crystal was monitored until the resonance frequency did not further change upon protein addition indicating full coverage of the binding sites (Fig. 5.2), which prevents any non-specific binding of cPC2. Rinsing with buffer results in only very little desorption of proteins showing the almost fully irreversible attachment of CPC1 to the membrane. 


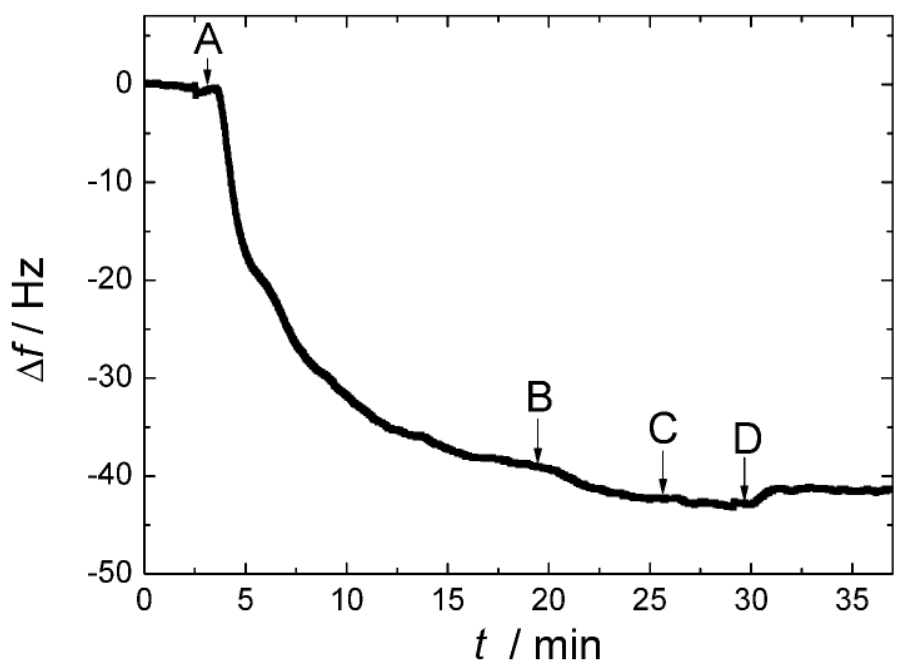

Fig. 5.2 Time course of the frequency shift of a $5 \mathrm{MHz}$ quartz plate functionalized with a solid supported membrane (octanethiol-DOPC/DOGS-NTA-Ni, 9:1) after addition of $0.87 \mu \mathrm{M}(\mathrm{A}), 0.98 \mu \mathrm{M}$ (B), $1.02 \mu \mathrm{M}$ (C) CPC1 in $\mathrm{Ca}^{2+}$ containing buffer. (D) indicates the time point of rinsing with buffer.

In $\mathrm{Ca}^{2+}$-free buffer ( $150 \mathrm{~mm} \mathrm{NaCl}, 20 \mathrm{mM} \mathrm{KH} 2 \mathrm{PO}_{4} / \mathrm{K}_{2} \mathrm{HPO}_{4}, 0.1 \mathrm{~mm}$ EDTA, $\mathrm{pH}$ 7.4) saturation of the binding sites was achieved at a CPC1 concentration of $(1.1 \pm 0.5) \mu \mathrm{M}$ resulting in an average decrease in resonance frequency of $-\Delta f_{\mathrm{e}}=(29 \pm 5) \mathrm{Hz}(\mathrm{n}=8)$. In the $\mathrm{Ca}^{2+}$-containing buffer (150 mM NaCl, $20 \mathrm{mM}$ HEPES, $1 \mathrm{mM} \mathrm{CaCl}$, $\mathrm{pH}$ 7.4) a mean final concentration of $\mathrm{cPC} 1$ of $(0.8 \pm 0.2) \mu \mathrm{M}$ was required, which produced an average shift in resonance frequency of $-\Delta f_{e}=(37 \pm 5) \mathrm{Hz}(\mathrm{n}=5)$. Simultaneously, for both buffer systems, a small change in the dynamic resistance $\Delta R$ was monitored demonstrating a slight viscoelastic contribution of $\mathrm{cPC} 1$ upon binding (in $\mathrm{Ca}^{2+}$-free buffer: $\Delta R=(7.9 \pm$ 0.6) $\Omega$, in $\mathrm{Ca}^{2+}$-containing buffer: $\left.\Delta R=(5.1 \pm 1.1) \Omega\right)$. 


\subsubsection{Interaction of cPC2 with cPC1 Immobilized on a Solid Supported Membrane}

Prior to the investigation of the $\mathrm{CPC} 2 / \mathrm{cPC} 1$ interaction, control experiments were performed to rule out any non-specific binding of cPC2. As a control, the protein ezrin harboring a histidine tag, was immobilized on the DOPC/DOGS-NTA-Ni (9:1) membrane. This protein does not specifically interact with cPC2. After immobilization of ezrin, which was observed by QCM, different concentrations of cPC2 were added up to $0.4 \mu \mathrm{M}\left(\mathrm{Ca}^{2+}\right.$-free buffer), and $0.2 \mu \mathrm{M}$ ( $\mathrm{Ca}^{2+}$ containing buffer). No significant change in resonance frequency was monitored independent of the chosen buffer demonstrating that non-specific binding of CPC2 to a protein decorated membrane can be excluded.

The specific interaction of cPC2 with $\mathrm{cPC} 1$ was also investigated by QCM after immobilization of cPC1 on the DOPC/DOGS-NTA-Ni membrane. cPC2 was added to the system, which results in an immediate decrease in resonance frequency. An addition of $0.34 \mu \mathrm{M} \mathrm{cPC} 2$ in $150 \mathrm{mM} \mathrm{NaCl}, 20 \mathrm{mM} \mathrm{KH} 2 \mathrm{PO}_{4} / \mathrm{K}_{2} \mathrm{HPO}_{4}, 0.1 \mathrm{mM}$ EDTA, pH 7.4 results in an immediate decrease in $\Delta f$ indicating the specific binding of cPC2 to CPC1 immobilized on the lipid membrane (Fig. 5.3) 


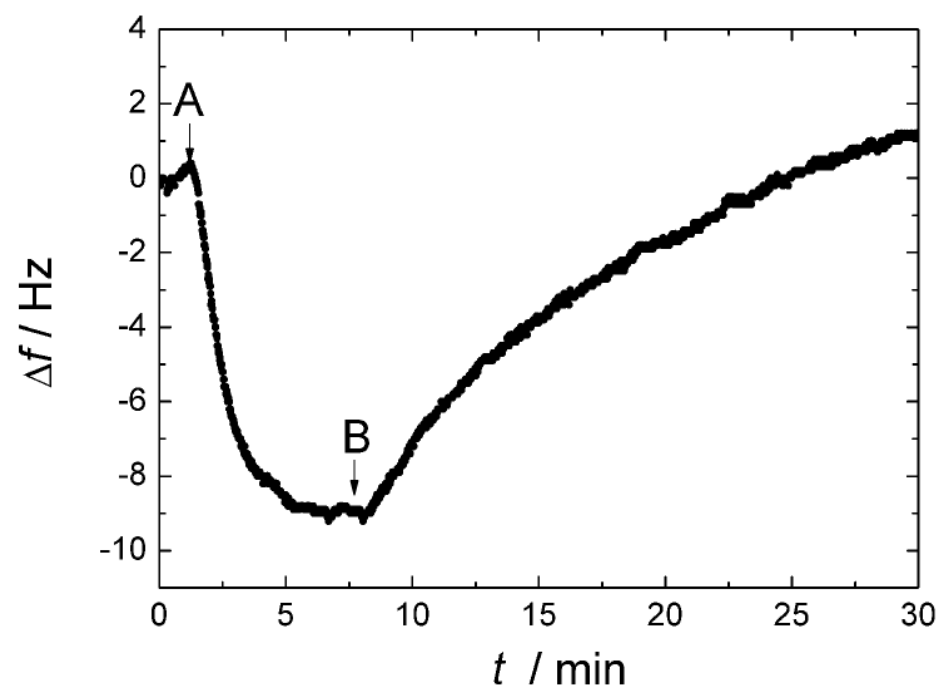

Fig. 5.3 Representative time course of the frequency shift upon addition of $0.34 \mu \mathrm{M} \mathrm{CPC} 2$ (A) to a solidsupported membrane (octanethiol-DOPC/DOGS-NTA-Ni, 9:1) with bound cPC1. (B) indicates the time point of rinsing with $\mathrm{Ca}^{2+}$-free buffer (150 mM NaCl, $20 \mathrm{mM} \mathrm{KH}_{2} \mathrm{PO}_{4} / \mathrm{K}_{2} \mathrm{HPO}_{4}, 0.1 \mathrm{mM}$ EDTA, pH 7.4).

A change in the dynamic resistance of $\Delta R=1.3 \Omega$ after protein addition was detected, characteristic for an almost negligible contribution of viscoelastic effects upon binding. After a constant frequency shift of $\Delta f_{\mathrm{e}}=-8.5 \mathrm{~Hz}$ was reached, the system was rinsed with buffer. An instant increase in resonance frequency to a value of $\Delta f_{\mathrm{e}}=0.5 \mathrm{~Hz}$ demonstrates that the binding of $\mathrm{CPC} 1$ to $\mathrm{CPC} 2$ is fully reversible. Reversibility of the interaction of cPC2 with cPC1 was found under both conditions, in the presence and absence of $\mathrm{Ca}^{2+}$.

\subsubsection{Binding Affinity of the $\mathrm{CPC} 1 / \mathrm{cPC} 2$ Interaction in the Absence and Presence of $\mathrm{Ca}^{2+}$}

To quantify the interaction of $\mathrm{cPC} 1$ with $\mathrm{cPC} 2$ as a function of $\mathrm{Ca}^{2+}$, the concentration dependent change in resonance frequency $\Delta f_{\mathrm{e}}$ upon addition of cPC2 to a $\mathrm{CPC} 1$ covered lipid bilayer was monitored by means of QCM. The $\Delta f_{\mathrm{e}}$ values were plotted versus the monomeric $\mathrm{CPC} 2$ concentration in solution and the parameters of the adsorption iso- 
therm based on the scaled particle theory (SPT) were fitted to the data (eq. 5.2) assuming the radius of $\mathrm{cPC} 2$ to be $a=1.7 \mathrm{~nm}:{ }^{13}$

$$
c_{\mathrm{cPC} 2}=\frac{K_{\mathrm{D}} \theta}{1-\theta} \exp \left[3 \frac{\theta}{1-\theta}+\left(\frac{\theta}{1-\theta}\right)^{2}\right]
$$

with $\theta=\frac{\Delta f_{\mathrm{e}}}{\Delta f_{\max }} . K_{\mathrm{D}}$ is the dissociation constant of the $\mathrm{CPC} 1 / \mathrm{CPC} 2$ interaction.

In $\mathrm{Ca}^{2+}$-free buffer ( $150 \mathrm{~mm} \mathrm{NaCl}, 20 \mathrm{mM} \mathrm{KH}_{2} \mathrm{PO}_{4} / \mathrm{K}_{2} \mathrm{HPO}_{4}, 0.1 \mathrm{~mm}$ EDTA, pH 7.4) $\mathrm{K}_{\mathrm{D}}$ was determined to be $(260 \pm 45) \mathrm{nM}$ with a maximal resonance frequency shift of $\Delta f_{\max }=$ $(35 \pm 5) \mathrm{Hz}$ (Fig. 5.4 A). However, in $\mathrm{Ca}^{2+}$ containing buffer $(150 \mathrm{mM} \mathrm{NaCl}, 20 \mathrm{mM}$ HEPES, $1 \mathrm{mM} \mathrm{CaCl}$, $\mathrm{pH} 7.4$ ) the binding constant of $\mathrm{CPC} 2$ to $\mathrm{CPC} 1$ was about three times larger exhibiting a dissociation constant of $K_{\mathrm{D}}=(81 \pm 30) \mathrm{nM}$, while the maximum shift in resonance frequency remains nearly the same $\left(\Delta f_{\max }=(37 \pm 5) \mathrm{Hz}\right.$, (Fig. $\left.5.4 \mathrm{~B}\right)$. 

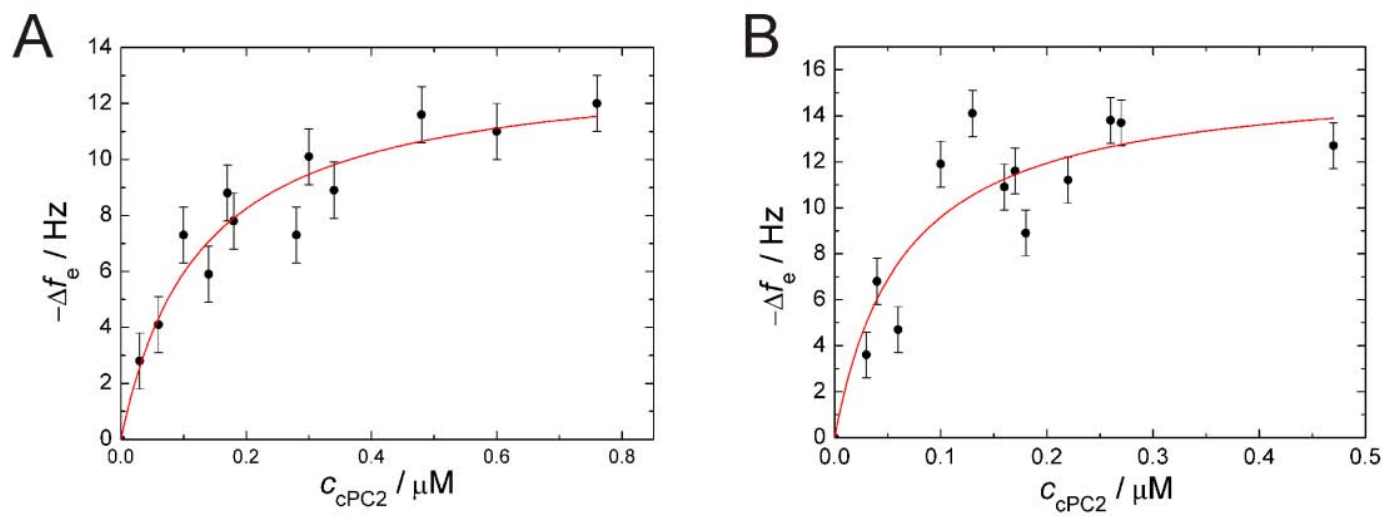

Fig. 5.4 (A) Adsorption isotherm of the cPC2/cPC1 interaction in $\mathrm{Ca}^{2+}$-free buffer $(150 \mathrm{mM} \mathrm{NaCl}, 20 \mathrm{mM}$ $\mathrm{KH}_{2} \mathrm{PO}_{4} / \mathrm{K}_{2} \mathrm{HPO}_{4}, 0.1 \mathrm{mM}$ EDTA, $\mathrm{pH}$ 7.4). The parameters of the adsorption isotherm (eq. 5.2) were fit to the resonance frequency shifts obtained from the QCM data. A dissociation constant of $(260 \pm 45) \mathrm{nM}$ was determined for the $\mathrm{cPC} 2 / \mathrm{CPC} 1$ complex with a maximal shift in resonance frequency of $\Delta f_{\max }=(35 \pm$ 5) Hz. (B) Adsorption isotherm of the cPC2/cPC1 interaction in $\mathrm{Ca}^{2+}$ containing buffer $(150 \mathrm{mM} \mathrm{NaCl}, 20$ mM HEPES, $1 \mathrm{mM} \mathrm{CaCl}$, pH 7.4). A dissociation constant of $(81 \pm 30) \mathrm{nM}$ for the cPC2/cPC1 complex was found with a maximal shift in resonance frequency of $\Delta f_{\max }=(37 \pm 5) \mathrm{Hz}$.

\subsubsection{Kinetics of the $\mathrm{cPC} 1 / \mathrm{cPC} 2$ Interaction in the Absence and Presence of $\mathrm{Ca}^{2+}$}

Besides the binding affinities, we asked the question, how the binding kinetics change as a function of the $\mathrm{Ca}^{2+}$-concentration. Thus, the rate constants of adsorption and desorption $k_{\text {on }}$ and $k_{\text {off, }}$ respectively were extracted from the time resolved changes in resonance frequency using the known $K_{D}$ values according to eq. 5.3:

$$
K_{\mathrm{D}}=\frac{k_{\text {off }}}{k_{\text {on }}}
$$

A representative result of the fitting routine to a plot of $\Delta f(t)$ obtained after the addition of $0.34 \mu \mathrm{M} \mathrm{cPC} 2$ to a membrane coated with $\mathrm{CPC} 1$ is depicted in Fig. 5.5. 


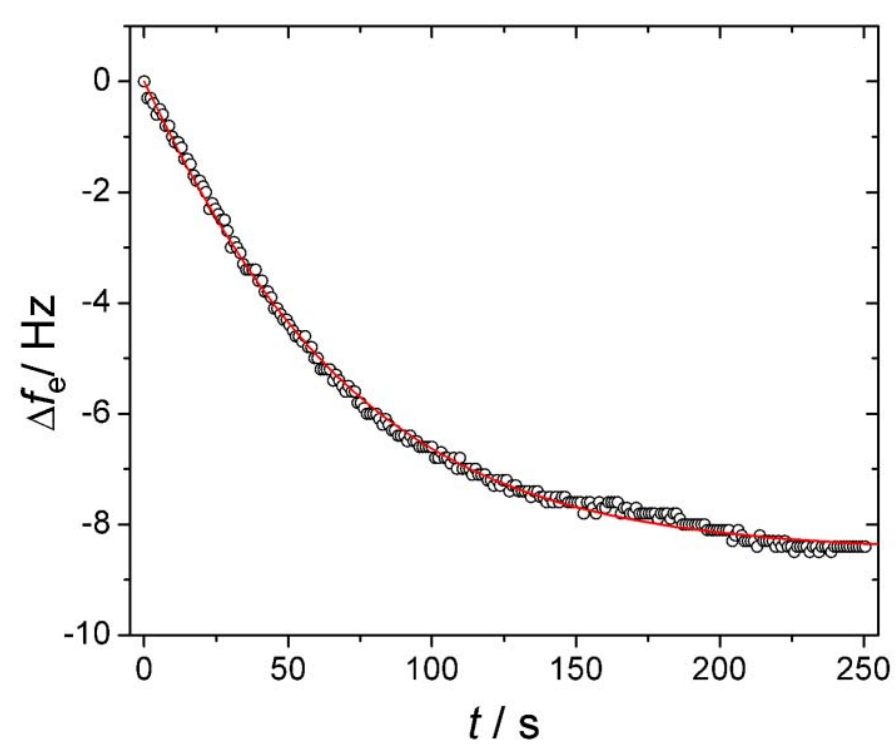

Fig. 5.5 Time dependent frequency shift after the addition of $0.34 \mu \mathrm{M} \mathrm{cPC} 2$ in $\mathrm{Ca}^{2+}$-free buffer $(150 \mathrm{mM}$ $\mathrm{NaCl}, 20 \mathrm{mM} \mathrm{KH}_{2} \mathrm{PO}_{4} / \mathrm{K}_{2} \mathrm{HPO}_{4}, 0.1 \mathrm{mM}$ EDTA, $\left.\mathrm{pH} 7.4\right)$. The adsorption rate constant $k_{\text {on }}$ was fit to the data based on the SPT model taking the mass transport to the surface into account $\left(k_{\mathrm{tr}}=(4.4 \pm 2.7) \cdot 10^{4} \mathrm{M}^{-}\right.$ $\left.{ }^{1} \cdot \mathrm{s}^{-1}(\mathrm{n}=6)\right)$. $k_{\text {on }}$ was determined to be $(2.5 \pm 0.7) \cdot 10^{4} \mathrm{M}^{-1} \cdot \mathrm{s}^{-1}(\mathrm{n}=6)$. From the obtained dissociation constant $K_{\mathrm{D}}=(260 \pm 45) \mathrm{nM}$ the rate constant of dissociation $k_{\text {off }}=(6.4 \pm 1.8) \cdot 10^{-3} \mathrm{~s}^{-1}(\mathrm{n}=6)$ was calculated.

The rate constants of adsorption and desorption in the absence of $\mathrm{Ca}^{2+}$ were determined to be $k_{\text {on }}=(2.5 \pm 0.7) \cdot 10^{4} \mathrm{M}^{-1} \cdot \mathrm{s}^{-1}$, and $k_{\text {off }}=(6.4 \pm 1.7) \cdot 10^{-3} \mathrm{~s}^{-1}$, while the transport rate constant reads $k_{\mathrm{tr}}=(4.4 \pm 2.7) \cdot 10^{4} \mathrm{M}^{-1} \cdot \mathrm{s}^{-1}(\mathrm{n}=6)($ Table 5.1$)$.

The rate constants of adsorption and desorption in the presence of $\mathrm{Ca}^{2+}$ were determined in the same manner resulting in $k_{\text {on }}=(2.1 \pm 0.5) \cdot 10^{4} \mathrm{M}^{-1} \cdot \mathrm{s}^{-1}$ and $k_{\text {off }}=(1.7 \pm 0.4) \cdot 10^{-3}$ $\mathrm{s}^{-1} \quad(\mathrm{n}=3)$. The transport rate constant could be determined to $k_{\mathrm{tr}}=(2.7 \pm 1.3) \cdot 10^{4} \mathrm{M}^{-1} \cdot \mathrm{s}^{-1}(\mathrm{n}=2)$ (Table 5.1). These results demonstrate that $k_{\mathrm{on}}$ is independent of the $\mathrm{Ca}^{2+}$-concentration, while the desorption process is 3-4 times faster in a $\mathrm{Ca}^{2+}$ free solution. 
Table 5.1 Rate constants $k_{\text {on }}$ and $k_{\text {off }}$ obtained from the changes in $\Delta \mathrm{f}$ as a function of time in the presence and absence of $\mathrm{Ca}^{2+}$. Fits to the data based on a SPT model were performed assuming a momoneric and trimeric form of cPC2 in the absence of $\mathrm{Ca}^{2+}$, respectively.

\begin{tabular}{c|c|c} 
& $k_{\text {on }} / \mathbf{1 0}^{\mathbf{4}} \mathbf{M}^{-\mathbf{1} \cdot \mathbf{s}^{-1}}$ & $k_{\text {off }} / \mathbf{1 0}^{-3} \mathbf{~ s}^{-\mathbf{1}}$ \\
\hline $\mathbf{1} \mathbf{~ m M ~ C a}^{2+}$ & $(2.1 \pm 0.5)$ & $(1.7 \pm 0.4)$ \\
\hline $\mathbf{0 . 1}$ mM EDTA (monomer) & $(2.5 \pm 0.7)$ & $(6.4 \pm 1.8)$ \\
\hline $\mathbf{0 . 1}$ mM EDTA (trimer) & $(7.7 \pm 2.3)$ & $(6.7 \pm 2.0)$
\end{tabular}

\subsection{Discussion}

By means of $\mathrm{CD}$ spectroscopy titration experiments a dissociation constant of $K_{\mathrm{D}}=(15 \pm 4 \mu \mathrm{M})$ could be extracted with an assumed binding stoichoimetry of $1: 1$. This binding stoichiometry was chosen, according to Petri et al., who found that the EF-hand domain of cPC2 harbors a single $\mathrm{Ca}^{2+}$ binding site (Fig. 5.6). ${ }^{25}$

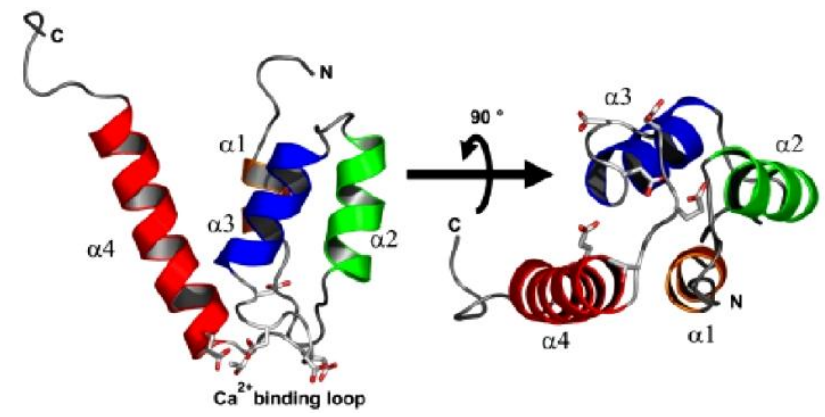

Fig. 5.6 Ribbon diagram of cPC2 EF-hand motif. ${ }^{25}$

The determined dissociation constant is in good agreement with the one determined by Célic et al. in 2008 by means of isothermal titration and in fairly good agreement with the one determined by Schumann et al. by means of fluorescence spectroscopy in 
2009.6,13 While Célic et al. found a dissociation constant of $K_{D}=(12.0 \pm 0.3 \mu \mathrm{M})$ with a $\mathrm{Ca}^{2+} / \mathrm{cPC} 2$ binding stoichoimetry of $\mathrm{x}=0.4$, Schumann et al. reported a $K_{\mathrm{D}}$ of $(68 \pm 2 \mu \mathrm{M})$ assuming a 1:1 stoichiometry. However, the cPC2 construct used by Célic et al. (Ile ${ }^{704}$ $\mathrm{Val}^{986}$ ) better resembles the $\mathrm{CPC} 2$ domain used in this work ( $\mathrm{Ile}^{680}-\mathrm{Val}^{968}$ ) compared to the shorted portion used by Schumann et al. (Ile $\left.{ }^{680}-\mathrm{Leu}^{796}\right)$. As shorter constructs (PC2EF, Lys ${ }^{704-M^{2}{ }^{800}}$ ) as used by Célic et al. also exhibited a larger dissociation constant of $K_{\mathrm{D}}=(214 \pm 3 \mu \mathrm{M})$, the construct length seems to been a crucial factor that needs to be considered, when comparing the cPC2 affinity to $\mathrm{Ca}^{2+}$.

By means of the quartz crystal microbalance technique, we were able to show that cPC2 specifically interacts with cPC1 with high affinity in the absence as well as in the presence of $\mathrm{Ca}^{2+}$. Using a model based on the scaled particle theory, binding constants were determined resulting in $K_{\mathrm{D}}\left(\mathrm{Ca}^{2+}\right)=(81 \pm 30) \mathrm{nM}$ and $K_{\mathrm{D}}($ EDTA $)=(260 \pm 45) \mathrm{nM}$. As these dissociation constants indicate the specificity of the binding, it is worth discussing the protein domains that might be involved in this interaction. It has been shown that the interaction between PC1 and PC2 is mediated through their C-termini and includes amino acid residues 742-986 of human PC2. ${ }^{26}$ It has further been suggested that coiled coil domains in the C-terminus of both PC2 and PC1 are involved in the interaction. By means of a yeast two-hybrid system Qian et al. ${ }^{10}$ demonstrated that cPC2 interacts with CPC1 and with itself. They predicated a coiled-coil domain of PC1 between residues 4214-4248 and one in PC2 between residues 772-796 with probabilities of more than $80 \%$ using the program COILS (version 2.0). ${ }^{9}$ However, another program (MULITCOIL) only scores probabilities of $5.4 \%$ (all scoring dimensions included). Even though there are a few known trimeric coiled coil structures, which show a total coiled coil probability of less than $10 \%$, the two different results highlight the ambiguity of coiled coil domain predictions. ${ }^{27,28}$ Our results clearly demonstrate that there is a specific interaction between the C-termini of PC1 and PC2. However, whether coiled coil domains drive this interaction, remains to be elucidated.

The results we obtained were analyzed under the assumption that $\mathrm{CPC} 2$ is monomeric in solution, which is corroborated by the hydrodynamic radii of $\mathrm{cPC} 2$ found in the 
presence of $\mathrm{Ca}^{2+} .^{13}$ However, Schumann et al ${ }^{13}$ also showed that the oligomerization state of $\mathrm{cPC} 2$ changes in the absence of $\mathrm{Ca}^{2+}$. They determined apparent molar masses and hydrodynamic radii of $\mathrm{CPC} 2$ in the presence and absence of $\mathrm{Ca}^{2+}$ and found that the ratio of the determined apparent molar masses of $\mathrm{cPC} 2$ suggests a dimer or trimer in the absence of $\mathrm{Ca}^{2+}$. These findings propose that the oligomerization state of cPC2 is probably a function of the $\mathrm{Ca}^{2+}$ concentration. However, Ferreira et al. reported a homotetrameric assembly of cPC2 $\left(\mathrm{Ile}^{680}-\mathrm{Val}^{968}\right)$ that was $\mathrm{Ca}^{2+}$ independent. ${ }^{29}$

With no excess of $\mathrm{Ca}^{2+}, \mathrm{Yu}$ et al. $^{14}$ found a trimeric form of $\mathrm{cPC} 2$ in solution as well as in the crystal structure. Moreover, they demonstrated that only the trimeric form of cPC2 interacts with $\mathrm{CPC} 1$, while a mutant (six residues critical for the trimer formation were simultaneously mutated to Ala) that fails to form a trimer, did not interact with $\mathrm{CPC}$. These findings were supported by molecular dynamic (MD) simulations performed by Zhu et al. in $2011 .{ }^{30}$ A structural model of the cPC2/cPC1 complex derived from their MD simulations is depicted in Fig. 5.7.

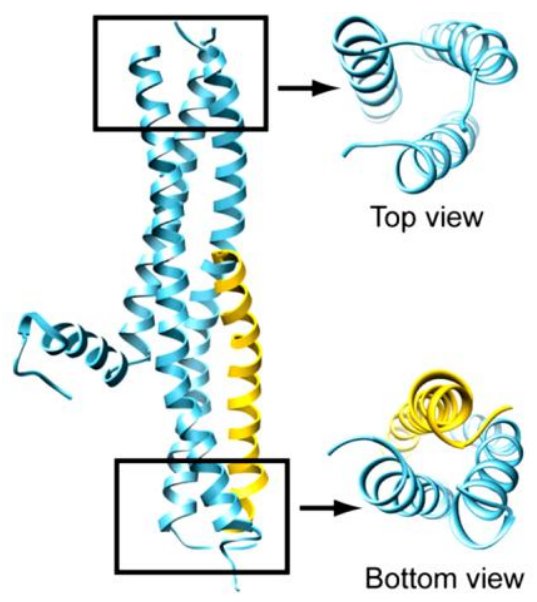

Fig. 5.7 Structural model of the cPC2/cPC2 coiled coil domain complex derived from MD simulation performed by Zhu et al..30

It remains still an open question, whether the disturbed interaction between the cPC2 mutant and cPC1 only occurs as a result of the non-existing trimer, or whether the mu- 
tation itself affects the ability of cPC2 to interact with cPC1. Another evidence of the preferential formation of trimers of $\mathrm{cPC} 2$ is given by the results using the program MULTICOIL, which predicts a probability for the formation of a trimer of about $80 \% .^{28}$

These results let us assume that $\mathrm{cPC} 2$ is trimeric in the absence of $\mathrm{Ca}^{2+}$, and binds as a trimer to $\mathrm{CPC} 1$. If we assume that only trimers exist that bind to $\mathrm{CPC} 1$, the dissociation constant for this reaction would change to $K_{D}(E D T A)=(87 \pm 15) n M$. Interestingly, in this case the affinity for both monomeric $\mathrm{CPC} 2\left(+\mathrm{Ca}^{2+}\right)$ and trimeric $\mathrm{cPC} 2\left(-\mathrm{Ca}^{2+}\right)$ to $\mathrm{cPC} 1$ is almost the same. From the overall maximum frequency changes it becomes, moreover, obvious that the total coverage is the same for $\mathrm{cPC} 2$ bound as a monomer $\left(\Delta f_{\max , \mathrm{Ca}^{2+}}\right.$ $=(37 \pm 5) \mathrm{Hz})$ or as the presumed trimer $\left(\Delta f_{\max , \mathrm{EDTA}}=(35 \pm 5) \mathrm{Hz}\right)$ to $\mathrm{CPC} 1$.

The assumption of cPC2 trimers in solution would also influence the rate constants of adsorption and desorption of the $\mathrm{cPC} 1 / \mathrm{cPC} 2$ interaction. If a trimer is proposed, the radius of the adsorbing particle changes by a factor of 2.15, while the bulk concentration of $\mathrm{cPC} 2$ as well as the dissociation constant is three times smaller. The resulting rate constants obtained by fitting the SPT model to the data are given in Table 1 as a function of the $\mathrm{Ca}^{2+}$-concentration. The adsorption rate constant becomes by a factor of 3.6 larger in the absence of $\mathrm{Ca}^{2+}$ than in its presence, while the desorption rate constant is about 4 times larger.

A conceivable scenario of the situation at the surface is depicted in Fig. 5.8. Binding of the $\mathrm{cPC} 2$ monomer to $\mathrm{CPC} 1$ on the membrane surface (Fig. $5.8 \mathrm{~A}$ ) in the presence of $\mathrm{Ca}^{2+}$ can occur in three independent steps with an apparent adsorption rate constant $k_{\mathrm{on} \text {,mono, }}$ which is the result of up to three individual rate constants $k_{\mathrm{on} 1}, k_{\mathrm{on} 2}$ and $k_{\mathrm{on} 3}$. The adsorption process of the assumed trimeric cPC2 in the absence of $\mathrm{Ca}^{2+}$ (Fig. 5.8 B) would oc-

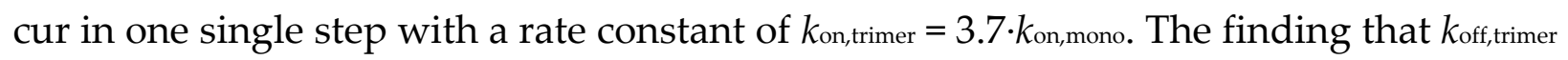
$=3.9 \cdot k_{\text {off,mono }}$ may indicate that the topologies of the $\mathrm{cPC} 1 / \mathrm{cPC} 2$ complex are different under the given conditions or that the desorption itself again occurs in multiple steps. 


\section{A}

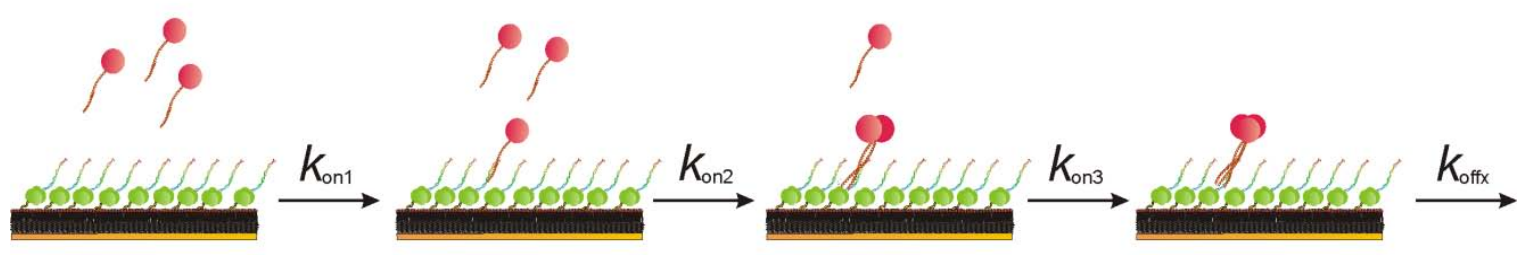

$\mathrm{B}$

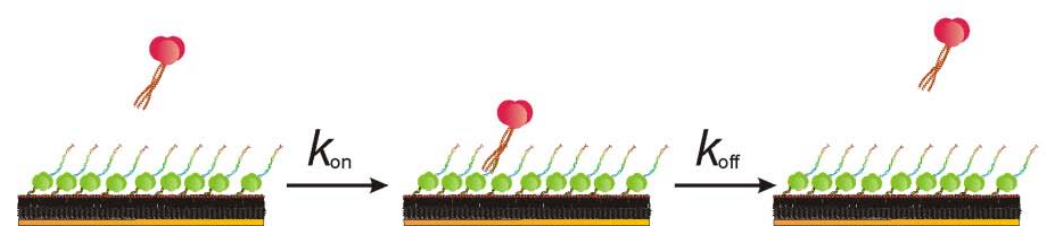

Fig. 5.8 Schematic drawing explaining the binding behavior of a cPC2 monomer (depicted in red) to CPC1 (shown in green) immobilized on a solid supported membrane (A) in $\mathrm{Ca}^{2+}$ containing buffer as a three step adsorption model. The assumed cPC2 trimer binds in a one step adsorption kinetics (B).

As yet, the SPT model appears to be a versatile tool to evaluate the rate constants of adsorption and desorption of protein binding taking also mass transport processes into account. Mass transport limitations are a result of the large number of binding sites at the membrane provided by cPC1. ${ }^{31}$ Effects from mass transport can be further reduced by adding an interaction inhibitor to the system or by increasing the flow rate in the QCM experiments. ${ }^{31,32}$ However, the flow rate of the QCM setup can only be increased within a small regime to ensure a good signal to noise ratio.

In conclusion, we were able to demonstrate that CPC2 binds to CPC1 in the absence as well as in the presence of $\mathrm{Ca}^{2+}$. That the dissociation constant of the interaction of cPC1 and $\mathrm{CPC} 2$ in the absence of $\mathrm{Ca}^{2+}$ is about three times larger than in its presence suggests that $\mathrm{CPC} 2$ trimers exist in the absence of $\mathrm{Ca}^{2+}$, which bind to $\mathrm{CPC} 1$.

\subsection{Co-workers}

Hoffmeister Hellen; Witzgall, Ralph; Janshoff, Andreas 


\subsection{References}

[1] Harris, P. C., and Torres, V. E. (2009) Polycystic kidney disease. Annu. Rev. Med. $60,321-337$.

[2] Wilson, P. D. (2001) Polycystin: new aspects of structure, function, and regulation. J. Am. Soc. Nephrol. 12, 834-845.

[3] Newby, L. J., Streets, A. J., Zhao, Y., Harris, P. C., Ward, C. J., and Ong, A. C. M. (2002) Identification, characterization, and localization of a novel kidney polycystin-1-polycystin-2 complex. J. Biol. Chem. 277, 20763-20773.

[4] Nauli, S. M., Alenghat, F. J., Luo, Y., Williams, E., Vassilev, P., Li, X., Elia, A. E. H., Lu, W., Brown, E. M., Quinn, S. J., Ingber, D. E., and Zhou, J. (2003) Polycystins 1 and 2 mediate mechanosensation in the primary cilium of kidney cells. Nat. Genet. 33, 129-137.

[5] Birnbaumer, L. (2009) The TRPC class of ion channels: a critical review of their roles in slow, sustained increases in intracellular $\mathrm{Ca}(2+)$ concentrations. Annu. Rev. Pharmacol. Toxicol 49, 395-426.

[6] Celić, A., Petri, E. T., Demeler, B., Ehrlich, B. E., and Boggon, T. J. (2008) Domain mapping of the polycystin-2 C-terminal tail using de novo molecular modeling and biophysical analysis. J. Biol. Chem. 283, 28305-28312.

[7] Luo, Y., Vassilev, P. M., Li, X., Kawanabe, Y., and Zhou, J. (2003) Native polycystin-2 functions as a plasma membrane $\mathrm{Ca}^{2+}$-permeable cation channel in renal epithelia. Mol. Cell. Biol. 23, 2600-2607.

[8] Vassilev, P. M., Guo, L., Chen, X. Z., Segal, Y., Peng, J. B., Basora, N., Babakhanlou, H., Cruger, G., Kanazirska, M., Ye Cp, Brown, E. M., Hediger, M. A., and Zhou, J. (2001) Polycystin-2 is a novel cation channel implicated in defective 
intracellular $\mathrm{Ca}(2+)$ homeostasis in polycystic kidney disease. Biochem. Biophys. Res. Commun. 282, 341-350.

[9] Tsiokas, L. (2009) Function and regulation of TRPP2 at the plasma membrane. Am. J. Physiol. Renal Physiol. 297, F1-9.

[10] Qian, F., Germino, F. J., Cai, Y., Zhang, X., Somlo, S., and Germino, G. G. (1997) PKD1 interacts with PKD2 through a probable coiled-coil domain. Nat. Genet 16, 179-183.

[11] Hanaoka, K., Qian, F., Boletta, A., Bhunia, A. K., Piontek, K., Tsiokas, L., Sukhatme, V. P., Guggino, W. B., and Germino, G. G. (2000) Co-assembly of polycystin-1 and -2 produces unique cation-permeable currents. Nature 408, 990-994.

[12] Cai, Y., Anyatonwu, G., Okuhara, D., Lee, K.-B., Yu, Z., Onoe, T., Mei, C.-L., Qian, Q., Geng, L., Wiztgall, R., Ehrlich, B. E., and Somlo, S. (2004) Calcium dependence of polycystin-2 channel activity is modulated by phosphorylation at Ser812. J. Biol. Chem. 279, 19987-19995.

[13] Schumann, F., Hoffmeister, H., Bader, R., Schmidt, M., Witzgall, R., and Kalbitzer, H. R. (2009) $\mathrm{Ca}^{2+}$-dependent conformational changes in a C-terminal cytosolic domain of polycystin-2. J. Biol. Chem. 284, 24372-24383.

[14] Yu, Y., Ulbrich, M. H., Li, M.-H., Buraei, Z., Chen, X.-Z., Ong, A. C. M., Tong, L., Isacoff, E. Y., and Yang, J. (2009) Structural and molecular basis of the assembly of the TRPP2/PKD1 complex. Proc. Natl. Acad. Sci. U.S.A 106, 11558-11563.

[15] Reiss, H., Frisch, H. L., and Lebowitz, J. L. (1959) Statistical Mechanics of Rigid Spheres. J. Chem. Phys. 31, 369.

[16] Janshoff, Galla, and Steinem (2000) Piezoelectric Mass-Sensing Devices as Bio sensors-An Alternative to Optical Biosensors? Angew. Chem. Int. Ed. Engl 39, 4004-4032.

[17] Steinem, C., and Janshoff, A. (2007) Piezoelectric Sensors, Springer Berlin Heidelberg. 
[18] Höök, F., and Kasemo, B. (2007) The QCM-D Technique for Probing Biomacromolecular Recognition Reactions, Ser. Chem. Sens. Biosens. 5, 425-447.

[19] Richter, R. P., Him, J. L. K., Tessier, B., Tessier, C., and Brisson, A. R. (2005) On the kinetics of adsorption and two-dimensional self-assembly of annexin A5 on supported lipid bilayers. Biophys. J. 89, 3372-3385.

[20] Lüthgens, E., Herrig, A., Kastl, K., Steinem, C., Reiss, B., Wegener, J., Pignataro, B., and Janshoff, A. (2003) Adhesion of liposomes: a quartz crystal microbalance study. Meas. Sci. Technol. 14, 1865-1875.

[21] Faiss, S., Kastl, K., Janshoff, A., and Steinem, C. (2008) Formation of irreversibly bound annexin A1 protein domains on POPC/POPS solid supported membranes. Biochim. Biophys. Acta 1778, 1601-1610.

[22] Janshoff, A., and Steinem, C. (2005) Label-free detection of protein-ligand interacttions by the quartz crystal microbalance. Method. Mol. Biol. 305, 47-64.

[23] Tellechea, E., Johannsmann, D., Steinmetz, N. F., Richter, R. P., and Reviakine, I. (2009) Model-independent analysis of QCM data on colloidal particle adsorption. Langmuir 25, 5177-5184.

[24] Bingen, P., Wang, G., Steinmetz, N. F., Rodahl, M., and Richter, R. P. (2008) Solvation effects in the quartz crystal microbalance with dissipation monitoring response to biomolecular adsorption. A phenomenological approach. Anal. Chem $80,8880-8890$.

[25] Petri, E. T., Celic, A., Kennedy, S. D., Ehrlich, B. E., Boggon, T. J., and Hodsdon, M. E. (2010) Structure of the EF-hand domain of polycystin-2 suggests a mechanism for Ca2+-dependent regulation of polycystin-2 channel activity. Proc. Natl. Acad. Sci. U.S.A 107, 9176-9181.

[26] Tsiokas, L., Kim, E., Arnould, T., Sukhatme, V. P., and Walz, G. (1997) Homoand heterodimeric interactions between the gene products of PKD1 and PKD2. Proc. Natl. Acad. Sci. U.S.A 94, 6965-6970. 
[27] Lupas, A. (1997) Predicting coiled-coil regions in proteins. Curr. Opin. Struct. Biol. 7, 388-393.

[28] Wolf, E., Kim, P. S., and Berger, B. (1997) MultiCoil: a program for predicting two- and three-stranded coiled coils. Protein Sci. 6, 1179-1189.

[29] Ferreira, F. M., Oliveira, L. C., Germino, G. G., Onuchic, J. N., and Onuchic, L. F. (2011) Macromolecular assembly of polycystin-2 intracytosolic C-terminal domain. Proc. Natl. Acad. Sci. U.S.A 108, 9833-9838.

[30] Zhu, J., Yu, Y., Ulbrich, M. H., Li, M.-h., Isacoff, E. Y., Honig, B., and Yang, J. (2011) Structural model of the TRPP2/PKD1 C-terminal coiled-coil complex produced by a combined computational and experimental approach. Proc. Natl. Acad. Sci. U.S.A 108, 10133-10138.

[31] Myszka, D. G. (1997) Kinetic analysis of macromolecular interactions using surface plasmon resonance biosensors. Curr. Opin. Biotechnol. 8, 50-57.

[32] O'Shannessy, D. J., and Winzor, D. J. (1996) Interpretation of deviations from pseudo-first-order kinetic behavior in the characterization of ligand binding by biosensor technology. Anal. Biochem 236, 275-283. 

Phosphorylation of $C$-terminal Poly-

\section{cystin-2 Influences the Interaction with PIGEA14: A QCM Study Based on Solid Supported Membranes}

\subsection{Abstract}

The transmembrane protein polycystin-2 (PC2) is generally located at the membrane of the endoplasmic reticulum. However, it is known to form a complex with polycystin-1 via its $C$-terminal domain at the plasma membrane, which is an essential regulatory step in cell proliferation and differentiation. A mechanism for PC2 trafficking was proposed, in which the interaction of PIGEA14 with PC2 is supposed to be dependent on the phosphorylation state of PC2. Here, we quantitatively investigated the interaction of PIGEA14 with the C-terminal part of polycystin-2 wild type (cPC2wt) and the pseudophosphorylated mutant (cPC2S812D) to elucidate the influence of PC2 phosphorylation on PIGEA14 binding. Solid supported membranes composed of octanethiol/1,2dioleoyl-sn-glycero-3-phosphocholine doped with the receptor lipid DOGS-NTA-Ni were used to attach PIGEA14 to the membrane via its hexahistidine tag. By means of the quartz crystal microbalance technique, binding affinities as well as kinetic constants of the interaction were extracted in a label-free manner by applying the scaled particle theory. The results show that the binding affinity of cPC2wt to PIGEA14 is twofold larger than that of cPC2S812D, which is in accordance with the proposed model. 


\subsection{Introduction}

The autosomal dominant polycystic kidney disease (ADPKD) is one of the most common inherited human renal diseases (prevalence of $\sim 1: 800$ ) characterized by the development of fluid filled renal cysts leading to end stage renal failure by the age of $60 .^{1,2}$ In $85 \%$ of the diagnosed patients, ADPKD is caused by mutations of the pkd1 gene. This gene encodes for an integral membrane protein called polycystin-1 (PC1). PC1 is composed of 4,300 amino acids ( 462 kDa) with 11 membrane spanning regions, an extracellular N-terminus and an intracellular C-terminal part. ${ }^{3}$ A second gene, pkd2, is mutated in $15 \%$ of all affected patients, encoding for the type 2 transmembrane protein polycystin-2 (PC2). ${ }^{4-6}$ PC2, composed of 968 amino acids ( 110 kDa), has six membrane spanning regions with the $C$ - and $N$-terminus located inside the cell. The $C$-terminus of PC2 (cPC2) harbors one accepted and one proposed coiled-coil domain between Glü 772 $\mathrm{Leu}^{796}$ and $\mathrm{Phe}^{839}$-Asp ${ }^{919}$, respectively, which facilitate protein-protein interactions and an EF-hand motif (Ala ${ }^{711}-\mathrm{Pro}^{797}$ ) as a potential $\mathrm{Ca}^{2+}$ binding site. ${ }^{7}$ Furthermore, the $\mathrm{C}$ terminal region exhibits a retention signal for the endoplasmic reticulum (ER) composed of a cluster of acidic amino acid residues $\left(\mathrm{Asp}^{790}-\mathrm{Ser}^{820}\right)$ and three known phosphorylation sites at $\operatorname{Ser}^{76}, \operatorname{Ser}^{801}$ and Ser ${ }^{812} .8,9$

While PC1 acts as a receptor sensitive to fluid shear stress, ${ }^{10,11}$ PC2 being a member of the transient receptor potential (TRP) ion channel family (TRPP2) ${ }^{12}$ functions as a non selective, $\mathrm{Ca}^{2+}$-permeable cation channel. ${ }^{13} \mathrm{PC} 2$ channel activity is regulated by numerous factors such as complex formation with PC1, ${ }^{14}$ PC2 oligomerization, $\mathrm{Ca}^{2+}$ concentration, $^{7}$ and phosphorylation. The formation of a heteromultimeric PC1-PC2 channel complex mediated through their C-terminal coiled-coil domains plays a crucial role in kidney development. Function and activation mechanism of this complex is highly dependent on its localization: In primary cilia, $\mathrm{Ca}^{2+}$-channel activity of PC2 is induced by fluid shear stress and regulates tissue development as suggested by Nauli 
et al. ${ }^{11}$ At the basolateral membrane, the PC1/PC2 complex can be implicated in cell-cell or cell-matrix adhesion..$^{15,16}$

Whereas PC1 is embedded in the plasma membrane, the subcellular localization of PC2 still remains a matter of discussion: depending on the cell line and cellular conditions, PC2 was found at the ER, the plasma membrane and primary cilium. ${ }^{17,18}$ Hanaoka et al. ${ }^{19}$ showed that PC1 facilitates the targeting of PC2 to the plasma membrane. Köttgen et al. ${ }^{20}$ discovered that the subcellular distribution of PC2 is influenced and directed by binding to phosphofurin acidic cluster sorting proteins PACS-1 and PACS-2. However, the mechanism of PC2 trafficking remains unclear as the PACS proteins only induce the retrieval of PC2 at the ER and Golgi compartment. Hidaka et al. ${ }^{21}$ identified PIGEA14 (polycystin-2 interactor, Golgi- and endoplasmic reticulum-associated protein with a mass of $14 \mathrm{kDa}$ ) as a PC2 interactor employing a yeast-two-hybrid screen with the C-terminus of PC2 (Fig. 6.1).

A

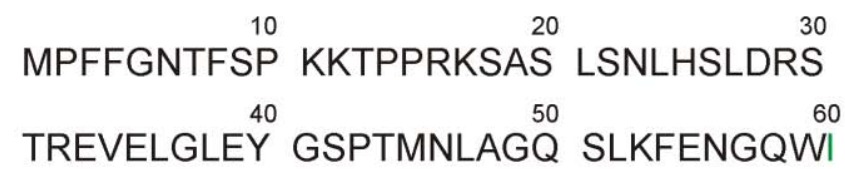

AETGVSGGVD RREVQRLRRR NQQLEEENNL

$\frac{100}{110}{ }^{100}{ }^{26}$

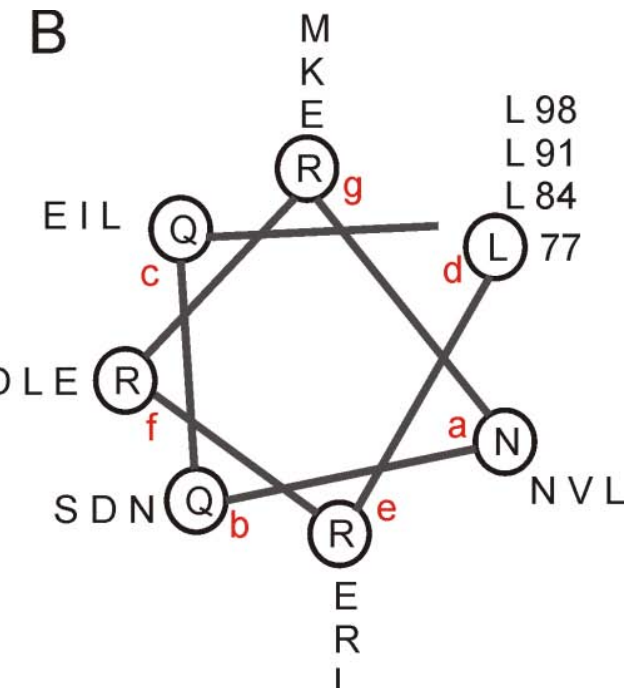

L

Fig. 6.1 (A) Amino acid sequence of PIGEA14 (Q9Y3M2) with the coiled coil domain (red square) and minimal interaction site for polycytsin-2 (green letters) (B) Helical wheel diagram of the coiled coil region (Arg77-Glu $\left.{ }^{98}\right)$. 
Co-expression of PC2 and PIGEA14 cDNA in HeLa cells showed a redistribution of these proteins from the ER to the trans-Golgi network. In another two hybrid screening with PIGEA14 as the bait protein, GM130 was isolated as an interaction partner, which is a component of the cis-Golgi compartment. These results implicate that PIGEA14 facilitates PC2 transport from the ER to the Golgi. ${ }^{22}$ Recently, it has been shown by means of NMR spectroscopy, circular dichroism and dynamic light scattering that the C-terminus of PIGEA14 harbors a coiled-coil domain between residue 73 and 100, ${ }^{23}$ which is essential for interacting with PC2..$^{24,21}$

Köttgen et al. ${ }^{17}$ suggested a mechanism of PC2 trafficking including PIGEA14 as the first PC2 interactor known to date, which is capable of mediating forward tracking of PC2. They hypothesized that PC2 is active at both the ER and the plasma membrane and that binding of PC2 to PACS-1/-2 after phosphorylation by casein kinase 2 (CK2) retrieves PC2 to the Golgi/ER. Dephosphorylation of PC2 releases PC2 from the PACSproteins and allows PC2 binding to PIGEA14. Thus, it is well conceivable that binding to PIGEA14 is governed by PC2 phosphorylation at Ser ${ }^{812}$. However, as yet there is no experimental evidence for this hypothesis.

To quantitatively investigate the interaction of PC2 with PIGEA14 and elucidate the influence of the phosphorylation of PC2, we made use of a recently established assay ${ }^{25}$ based on the quartz crystal microbalance (QCM) technique 26,27 , which allows us to monitor protein-protein complex formation in a time resolved and label free manner. Solid supported membranes doped with the receptor lipid DOGS-NTA-Ni were used, which allow to specifically bind the hexahistidine (His6)-tagged protein PIGEA14. The interaction of the C-terminal part of PC2 wild type (cPC2wt) as well as a mimic of the phosphorylated cPC2 protein, cPC2S812D was quantified by means of the QCM technique. From our results, we conclude that the phosphorylation of cPC2 significantly alters its binding affinity to PIGEA14, which supports the proposed model of PC2 recruitment to the plasma membrane. 


\subsection{Results}

\subsubsection{Expression, Purification and Characterization of PIGEA14}

PIGEA14 was recombinantly expressed in E. coli with a hexahistidine tag at the $C$ terminus. To increase the yield of soluble, active protein and minimize the formation of inclusion bodies, protein expression was performed at a temperature of $30^{\circ} \mathrm{C}$ rather than $37^{\circ} \mathrm{C} .{ }^{88,29}$ Protein purification by affinity chromatography using a Ni-iminodiacetic acid (Ni-IDA) agarose column was achieved with $50 \mathrm{mM}$ EDTA instead of imidazole, as the use of imidazole resulted in protein denaturation/precipitation during dialysis against storage buffer. Denaturation/precipitation of PIGEA14 after elution with imidazole containing buffer was confirmed by means of quartz crystal microbalance (QCM) binding experiments performed after dialysis in storage buffer. No binding of the hexahistidine tagged protein was monitored in terms of changes in resonance frequency after protein addition to a DOGS-NTA-Ni containing membrane, indicating that functional protein was not obtained. Of note, purification of the PIGEA14 cell lysate was unsuccessful if a commonly applied Ni-nitrilotriacetic acid (Ni-NTA) agarose was used; only Ni-IDA agarose led to successful protein purification. IDA chelates $\mathrm{Ni}^{2+}$ ions with lower affinity than NTA and thus PIGEA14 together with the $\mathrm{Ni}^{2+}$ can be eluted more efficiently with EDTA containing buffer. ${ }^{30}$ High purity of the protein solution was documented by SDS-PAGE showing PIGEA14 at about 17 kDa (Fig. 6.2, SDS-PAGE, lane E). Western Blot analysis using a pentahistidine antibody that recognizes the hexahistidine tag (anti His5, Qiagen, Hilden, Germany) confirmed the presence of an accessible histidine tag of PIGEA14 (Fig. 6.2, WB, lane E). 


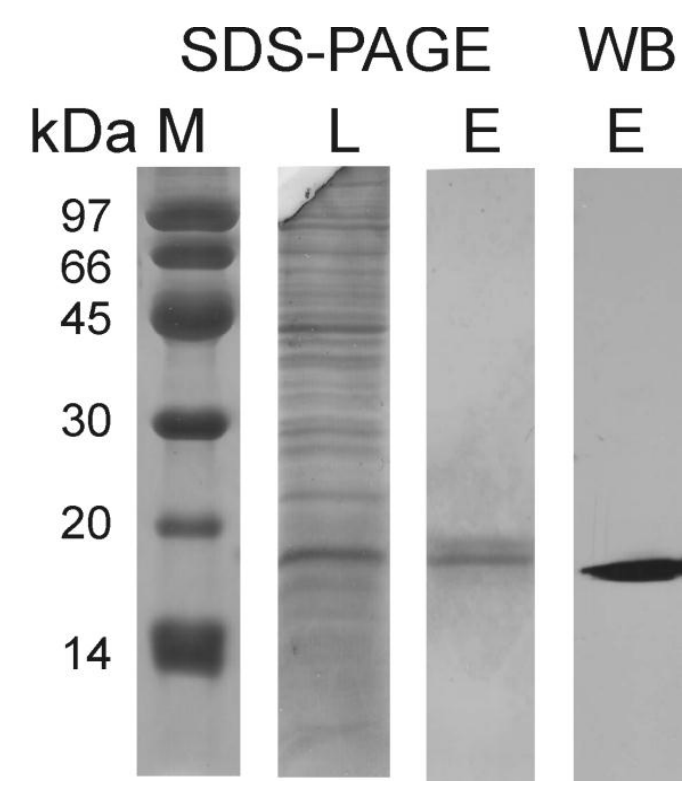

Fig. 6.2 SDS gels and Western blot analysis of PIGEA14 protein fractions. The Coomassie stained SDS gel shows the molecular marker (M), cell lysate (L) and elution fraction (E) of PIGEA14. According to the SDS gel and Western blot analysis (WB, anti Hiss) the molecular weight of PIGEA14 is approx. $17 \mathrm{kDa}$.

\subsubsection{Analysis of cPC2wt and cPC2S812D}

By means of circular dichroism (CD), we analyzed the secondary structure of both CPC2wt and S812D mutant to ensure that the pseudophosphorylation does not alter the protein structure. CD spectra of cPC2wt and cPC2S812D (Fig. 6.3) demonstrate that no significant difference between the two proteins' secondary structures can be deduced. 


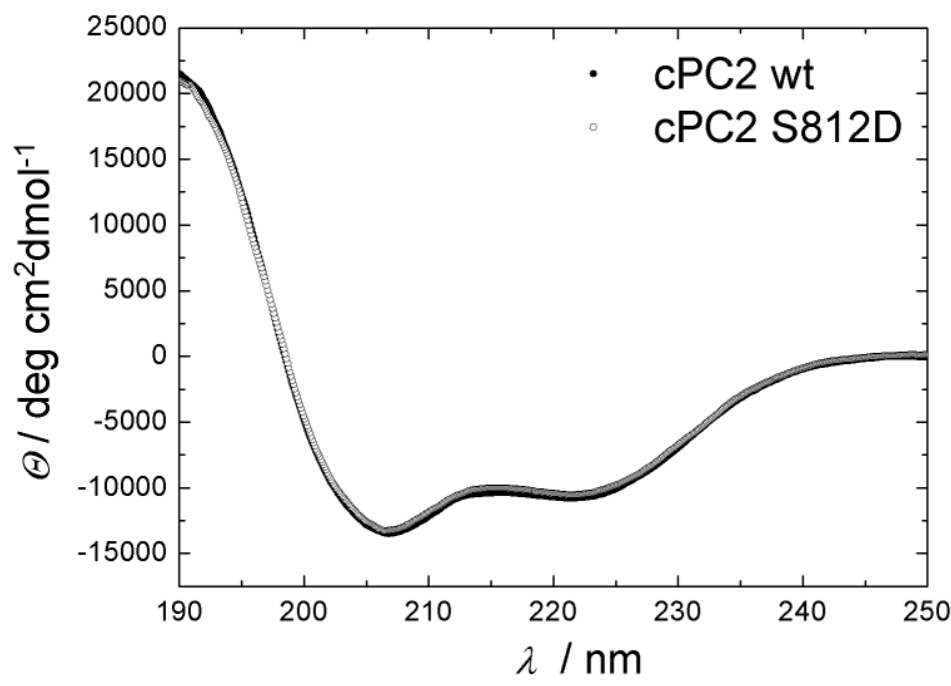

Fig. 6.3 CD spectra of cPC2wt and cPC2S812D mutant recorded in $20 \mathrm{mM} \mathrm{KH}_{2} \mathrm{PO}_{4} / \mathrm{K}_{2} \mathrm{HPO}_{4}, 1 \mathrm{~mm}$ EDTA, pH 7.4. Data evaluation assigns $36 \%$ helix, $13 \%$ strand, $16 \%$ turn and $35 \%$ unordered structures.

The different secondary structural elements of both proteins were calculated using DICHROWEB (CDSSTR algorithm, reference set 7). $36 \% \alpha$-helix, $13 \% \beta$-strand, $16 \%$ turns and $35 \%$ random coil was determined. The calculated $\alpha$-helicity is in good agreement with the $\alpha$-helical content of $34 \%$ determined by Celic et al. ${ }^{8}$ and $30 \%$ determined by Schumann et al. ${ }^{31}$ for $\mathrm{CPC} 2 \mathrm{wt}$ using CD spectroscopy in $\mathrm{Ca}^{2+}$ free buffer.

\subsubsection{Immobilization of PIGEA14 on Solid Supported Membranes}

To be able to investigate the interaction of PIGEA14 with cPC2wt and cPC2S812D, respectively, PIGEA14 was first bound to a hybrid solid supported membrane containing DOGS-NTA-Ni via its histidine tag. ${ }^{25}$ Hybrid solid supported membranes composed of DOPC doped with $10 \mathrm{~mol} \%$ of DOGS-NTA-Ni were used to ensure an almost irreversible immobilization of the hexahistidine-tagged PIGEA14 and maximal surface coverage. In contrast to a covalent attachment of PIGEA14 to the surface, binding of the protein to a solid supported membrane allows for its lateral mobility, which might influence the interaction with $\mathrm{CPC} 2$ as a result of conceivable protein reorganization. To 
investigate the specificity of PIGEA14 binding to DOGS-NTA-Ni, control experiments with neat DOPC membranes were performed. The addition of $0.3 \mu \mathrm{M}$ PIGEA14 does not result in any non-specific binding, i.e. no shift in resonance frequency of the quartz crystal was observed (Fig. 6.4 A). However, the addition of $0.65 \mu \mathrm{M}$ PIGEA14 to a DOPC/DOGS-NTA-Ni (9:1) hybrid membrane results in a significant decrease in resonance frequency of the $5 \mathrm{MHz}$ quartz plate. The change in resonance frequency $(\Delta f)$ was monitored until the resonance frequency does not further change upon protein addition indicating maximum protein binding. Rinsing with buffer only leads to a small increase in resonance frequency, indicating an almost irreversible binding of PIGEA14 (Fig. 6.4 B).
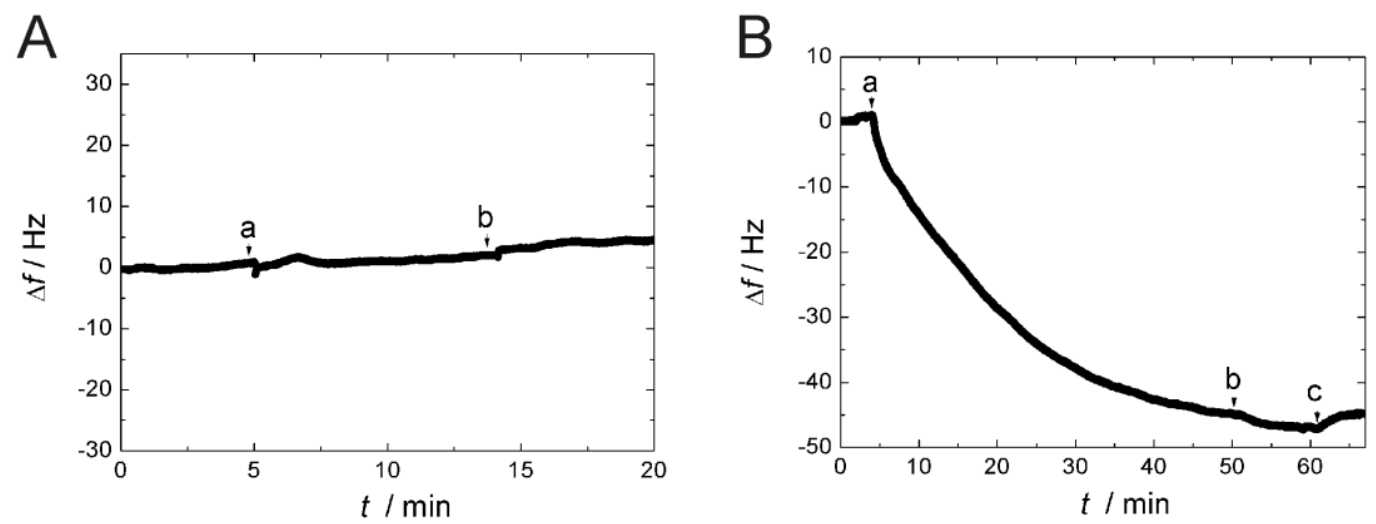

Fig. 6.4 (A) Time course of the frequency shift after addition of (a) $0.3 \mu \mathrm{M}$ PIGEA14 to a solid supported membrane composed of DOPC and (b) buffer rinsing. Buffer: $500 \mathrm{mM} \mathrm{NaCl}, 20 \mathrm{mM}$ TRIS/HCl, pH 8.0. (B) Representative time course of the frequency shift after addition of (a) $0.65 \mu \mathrm{M}$ and (b) $0.73 \mu \mathrm{M}$ PIGEA14 to a solid supported membrane (OT-DOPC/DOGS-NTA-Ni, 9:1). (c) indicates the time point of buffer rinsing.

A PIGEA14 concentration of $0.8 \mu \mathrm{M}$ resulted in an average shift in resonance frequency of $-\Delta f_{\mathrm{e}}=(69 \pm 19) \mathrm{Hz}(\mathrm{n}=16)$, whereas the increase in resonance frequency after rinsing with buffer was below $10 \%$ in all cases $\left(\Delta f_{\mathrm{e}}=(5 \pm 3) \mathrm{Hz}, \mathrm{n}=16\right)$. 


\subsubsection{Interaction of cPC2wt and cPC2S812D with PIGEA14}

Prior to the investigation of the $\mathrm{cPC} 2 \mathrm{wt} / \mathrm{cPC} 2 \mathrm{~S} 812 \mathrm{D}$ interaction with PIGEA14, control experiments were performed to rule out any non-specific binding of CPC2 to a proteincovered DOPC/DOGS-NTA-Ni membrane. The hexahistidine-tagged protein ezrin, a protein, which does not specifically interact with $\mathrm{CPC} 2$, was bound to the hybrid bilayer composed of DOPC/DOGS-NTA-Ni (9:1). cPC2wt was added up to a concentration of $0.4 \mu \mathrm{M}$ with no significant change in resonance frequency. This result demonstrates that non-specific binding of $\mathrm{cPC} 2$ to a protein-covered membrane can be excluded.

The specific interaction of PIGEA14 with cPC2 wt and cPC2S812D mutant was monitored by QCM. Different cPC2 concentrations were added, which resulted in an immediate decrease in resonance frequency. A characteristic time-dependent decrease in resonance frequency after the addition of cPC2wt $(0.27 \mu \mathrm{M})$ to a PIGEA14-covered membrane is shown in Fig. 6.5. 


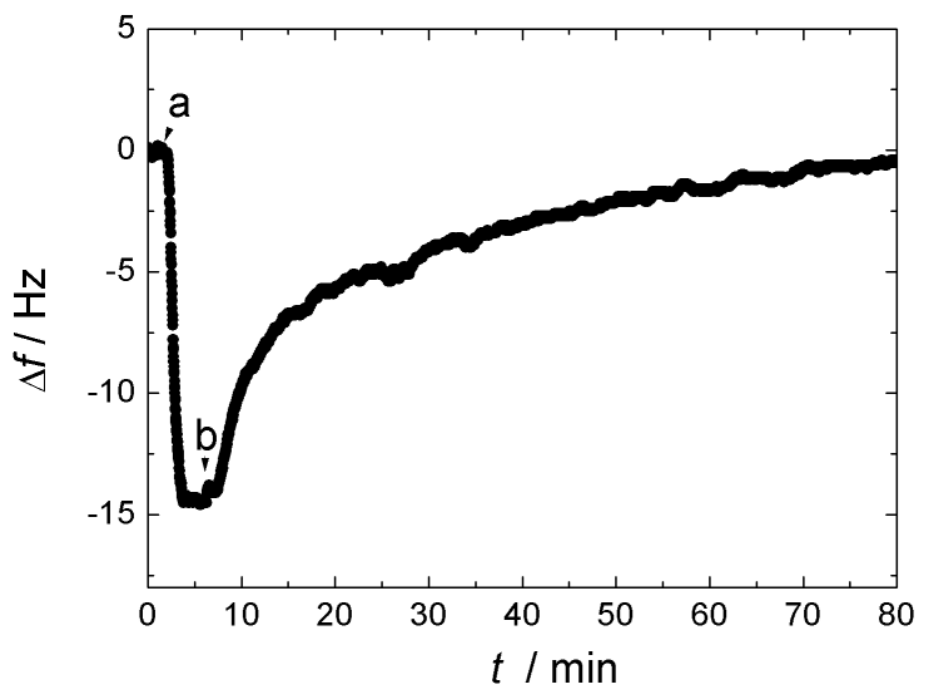

Fig. 6.5 Representative time course of the frequency shift after addition of (a) $0.27 \mu \mathrm{M} \mathrm{CPC} 2 w \mathrm{t}$ to a solid supported membrane (OT-DOPC/DOGS-NTA-Ni, 9:1) with bound PIGEA14. (b) indicates the time point of buffer rinsing ( $150 \mathrm{mM} \mathrm{NaCl}, 20 \mathrm{mM} \mathrm{KH}_{2} \mathrm{PO}_{4} / \mathrm{K}_{2} \mathrm{HPO}_{4}, 1$ mM EDTA, pH 7.4).

After reaching a constant frequency value, the shift in resonance frequency at equilibrium was read out to be $\Delta f_{\mathrm{e}}=-14.5 \mathrm{~Hz}$. As the dynamic resistance $\Delta R$ does not change during the binding event, it can be assumed that viscoelastic contributions are negligible. Subsequently after a constant frequency was reached, the system was rinsed with phosphate buffer, which led to an increase in resonance frequency of $\Delta f_{\mathrm{e}}=14.0 \mathrm{~Hz}$ indicating that $\mathrm{cPC} 2$ wt binding to PIGEA14 is fully reversible. For all QCM experiments, the binding process turned out to be almost fully reversible.

\subsubsection{Binding Affinity and Kinetics of cPC2wt and cPC2S812D}

To quantify the interaction of PIGEA14 with cPC2wt and cPC2S812D mutant, the change in resonance frequency at equilibrium $\Delta f_{\mathrm{e}}$ was determined as a function of cPC2 concentration. The obtained $\Delta f_{\mathrm{e}}$ values were plotted vs. the $\mathrm{cPC} 2$ concentration and the parameters of the adsorption isotherm based on the scaled particle theory (SPT) were fit to the data (eq. 6.1): 


$$
c_{\mathrm{cPC} 2}=\frac{K_{\mathrm{D}} \theta}{1-\theta} \exp \left[3 \frac{\theta}{1-\theta}+\left(\frac{\theta}{1-\theta}\right)^{2}\right]
$$

with $\theta=\frac{\Delta f_{\mathrm{e}}}{\Delta f_{\max }}$. The radius of $\mathrm{CPC} 2$ was assumed to be $a=1.7 \mathrm{~nm} \cdot{ }^{31}$ A dissociation constant of $K_{\mathrm{D}}=(64 \pm 22) \mathrm{nM}$ was determined for the PIGEA14/cPC2wt interaction (Fig. 6.6 A). However, the affinity of the pseudophosphorylated mutant cPC2S812D to PIGEA14 was two times lower with a dissociation constant of $K_{\mathrm{D}}=(137 \pm 41) \mathrm{nM}$ (Fig. 6.6 B).
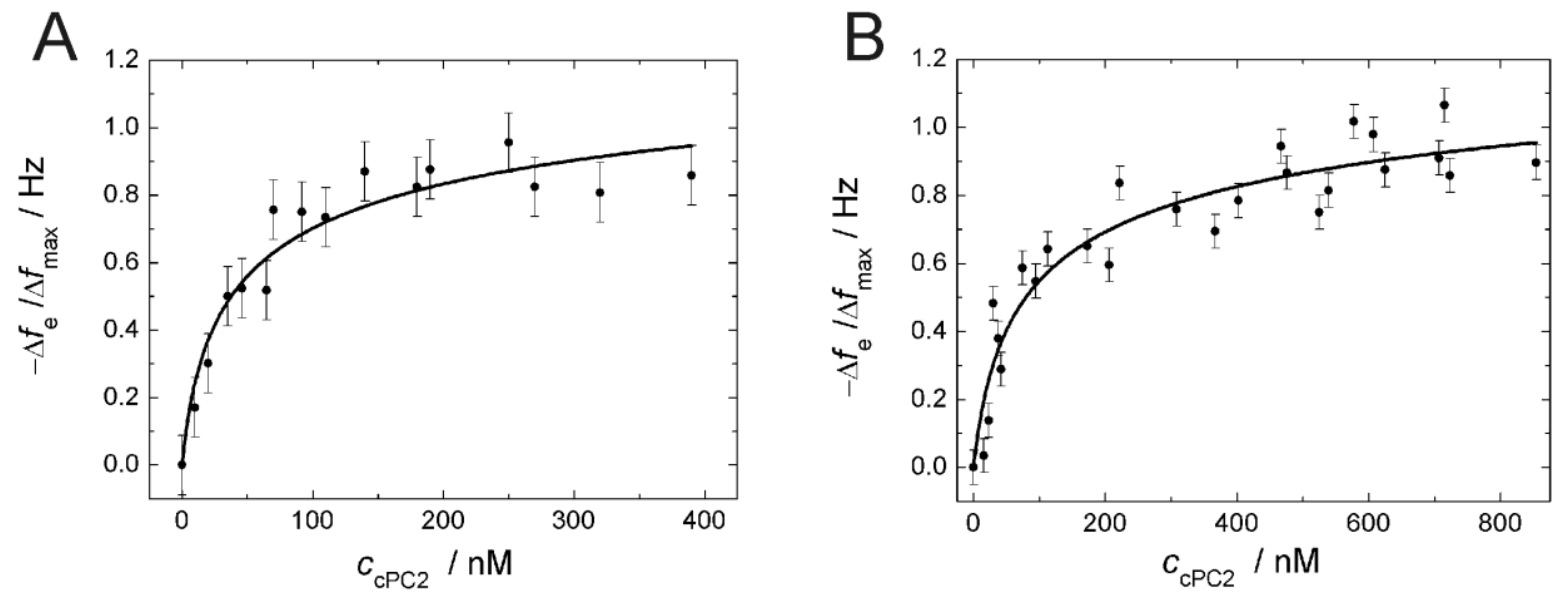

Fig. 6.6 Adsorption isotherms of (A) cPC2wt and (B) cPC2S812D interacting with PIGEA14. The parameters of eq. 6.1 were fit to the data. A dissociation constant of $K_{\mathrm{D}}=(64 \pm 22) \mathrm{nM}$ was determined for the cPC2wt/PIGEA14 interaction, while a $K_{\mathrm{D}}=(137 \pm 41) \mathrm{nM}$ was found for the cPC2S812D/PIGEA14 complex.

In addition to the binding affinities, we raised the question, whether the binding kinetics are also influenced by $\mathrm{cPC} 2$ pseudophosphorylation. Using the known value of $K_{\mathrm{D}}$, we determined the rate constant of the $\mathrm{cPC} 2$ adsorption $k_{\text {on }}$ and desorption $k_{\text {off. }} k_{\text {on }}$ was obtained by a fitting routine of $\Delta f(t)$ based on the scaled particle theory; $k_{\text {off }}$ was calculated from the known dissociation constant according to eq. (eq. 6.2): 


$$
K_{\mathrm{D}}=\frac{k_{\mathrm{off}}}{k_{\mathrm{on}}}
$$

A characteristic result of the fitting routine of the time dependent frequency shift $\Delta f(t)$ after addition of $0.31 \mu \mathrm{M}$ cPC2S812D to a DOGS-NTA-Ni/DOPC membrane covered with PIGEA14 is shown in Fig. 6.7.

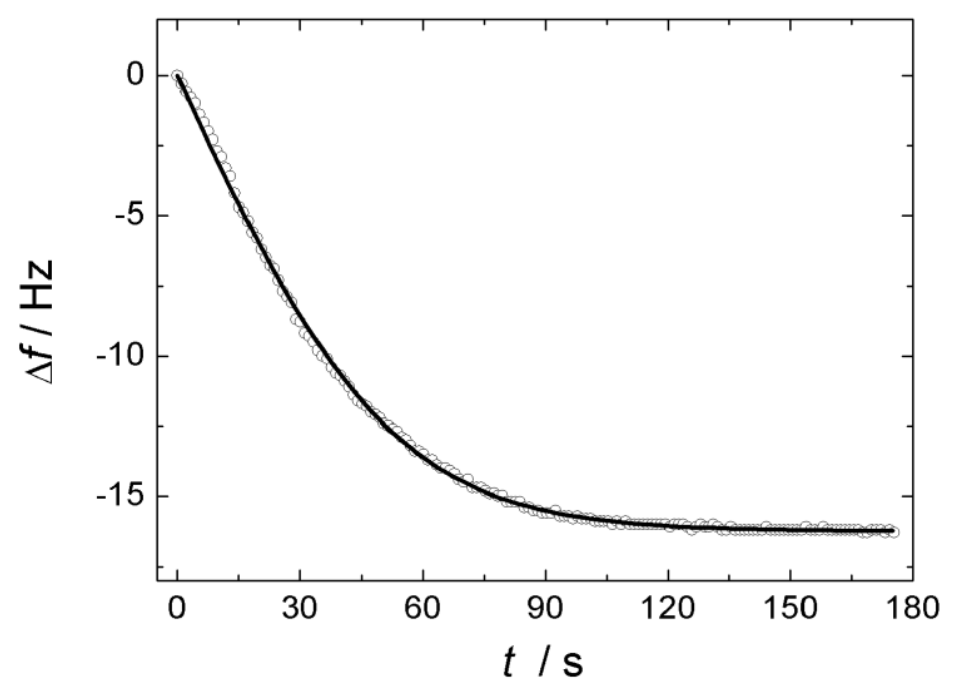

Fig. 6.7 Representative time dependent shift of resonance frequency after the addition of $0.31 \mu \mathrm{M}$ cPC2S812D to a membrane composed of OT-DOGS-NTA-Ni/DOPC, 9:1 with bound PIGEA14. The adsorption rate constant $\left(k_{\mathrm{on}}\right)$ was fit to the data according to a SPT model taking mass transport effects $\left(k_{\mathrm{tr}}\right)$ into account. The rate constant of dissociation $k_{\text {off }}$ was calculated using the corresponding $K_{\mathrm{D}}$. The rate constants were determined to be $k_{\text {on }}=(6.9 \pm 2.4) \cdot 10^{4} \mathrm{M}^{-1} \cdot \mathrm{s}^{-1}, k_{\text {off }}=(10.9 \pm 4.2) \cdot 10^{-3} \mathrm{~s}^{-1}, k_{\mathrm{tr}}=(1.6 \pm 0.6) \cdot 10^{4} \mathrm{M}^{-1} \cdot \mathrm{s}^{-1}$.

An adsorption rate constant of $k_{\mathrm{on}}=(6.9 \pm 2.4) \cdot 10^{4} \mathrm{M}^{-1} \cdot \mathrm{s}^{-1}$, a desorption rate constant of $k_{\text {off }}=(10.9 \pm 4.2) \cdot 10^{-3} \mathrm{~s}^{-1}$, along with the rate constant of mass transport $k_{\mathrm{tr}}=(1.6 \pm 0.6) \cdot 10^{4} \mathrm{M}^{-1} \cdot \mathrm{s}^{-1}$ were determined $(\mathrm{n}=5)$. The rate constants of the PIGEA14/cPC2wt interaction were determined in the same manner resulting in 
$k_{\text {on }}=(10 \pm 3) \cdot 10^{4} \mathrm{M}^{-1} \cdot \mathrm{s}^{-1}, k_{\text {off }}=(6.6 \pm 2.1) \cdot 10^{-3} \mathrm{~s}^{-1}$ and $k_{\mathrm{tr}}=(4.1 \pm 3.6) \cdot 10^{4} \mathrm{M}^{-1} \cdot \mathrm{s}^{-1}(\mathrm{n}=5$, data not shown) All kinetic constants of the PIGEA14 interaction with cPC2wt and cPC2S812D mutant together with the respective dissociation constants $K_{D}$ are summarized in Table 6.1 .

Table 6.1 Rate constants of adsorption $\left(k_{\circ}\right)$ and desorption $\left(k_{\text {off }}\right)$ together with the rate constant of mass transport $\left(k_{\mathrm{tr}}\right)$ of the PIGEA14 interaction with cPC2wt and cPC2S812D. Fits to the data obtained from the time dependent shift in resonance frequency were performed using a model based on the SPT theory.

\begin{tabular}{c|c|c|c|c} 
PIGEA14 + & $k_{\text {on }} / \mathbf{M}^{-1} \cdot \mathbf{s}^{-1}$ & $k_{\text {off }} / \mathbf{s}^{-1}$ & $k_{\mathrm{tr}} / \mathbf{M}^{-\mathbf{1}} \cdot \mathbf{s}^{-\mathbf{1}}$ & $K_{\mathbf{D}} / \mathbf{n M}$ \\
\hline cPC2wt & $(10 \pm 3) \cdot 10^{4}$ & $(6.7 \pm 2.1) \cdot 10^{-3}$ & $(4.1 \pm 3.6) \cdot 10^{4}$ & $(64 \pm 22)$ \\
\hline cPC2S812D & $(6.9 \pm 2.4) \cdot 10^{4}$ & $(10.9 \pm 4.2) \cdot 10^{-3}$ & $(1.6 \pm 0.6) \cdot 10^{4}$ & $(137 \pm 41)$
\end{tabular}

\subsection{Discussion}

A mechanism of PC2 trafficking has been proposed by Köttgen et al.,17 which is based on the previously shown phosphorylation-dependent interaction of PC2 with the proteins PACS-1/-2 and which suggests that the PC2-PIGEA14 interaction is also influenced by PC2 phosphorylation at Ser ${ }^{812}$ (Fig. 6.8). Phosphorylation of TRP channels including TRPP2 (PC2) ${ }^{32}$ is a general mechanism, by which these proteins are regulated..$^{33}$ For example, Cai et al. ${ }^{34}$ discovered that the PC2 channel activity is $\mathrm{Ca}^{2+}$-dependent and that it can be modulated by phosphorylation of Ser ${ }^{812}$. In recent studies, Streets et al. ${ }^{9}$ found that phosphorylation at Ser ${ }^{801}$ governs the channel activity of PC2 and influences cell growth. 


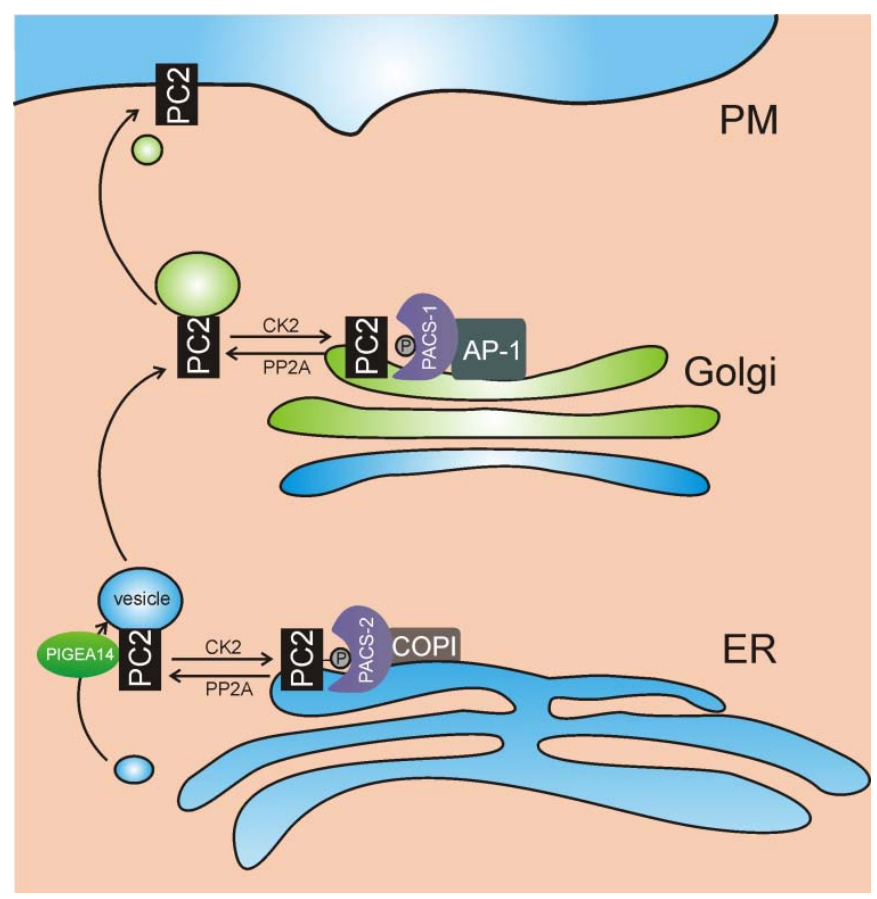

Fig. 6.8 Postulated mechanism of PC2 trafficking. PC2, phosphorylated by casein kinase 2 (CK2) at Ser ${ }^{812}$, is retrieved at the endoplasmic reticulum (ER) via binding to PACS-2/COPI. Desphosphorylation of PC2 by protein phosphatase $2 \mathrm{~A}$ (PP2A) facilitates its translocation to the Golgi compartment. This transport is governed by binding to PIGEA14. Retrieval at the Golgi compartment is mediated by PACS-1/adaptin protein-1 (AP-1) to phosphorylated PC2. Dephosphorylation and further unknown factors facilitate PC2 trafficking to the plasma membrane.

The interaction partner under investigation in this study, namely PIGEA14, is the first protein known to date that facilitates forward trafficking of PC2 from the ER to the Golgi compartment. ${ }^{21}$ This process might be influenced by the phosphorylation state of PC2. We were able to quantitatively analyze the interaction of the $C$-terminal domain of PC2 (cPC2) with PIGEA14 bound to a lipid membrane as a function of CPC2 phosphorylation at Ser ${ }^{812}$ by using the mutant cPC2S812D. With our chosen setup, the proteinprotein interaction is limited to a planar two-dimensional geometry similar to the situation found at cellular membranes. The two dimensional interaction site influences the binding behavior of the proteins, as one interaction partner is localized on a surface, which reduces its translational and rotational degrees of freedom leading to altered binding affinities.$^{35}$ Replacing a Ser, or Thr residue by a Glu or Asp residue is a common method to introduce pseudophosphorylations in proteins. However, even single 
point mutations can considerably influence the protein structure and can in principle interfere with the protein's ability to bind specific interaction partners. In a recent study by Bosk et al. ${ }^{36}$, it was shown that a single point mutation at the phosphorylation site of the protein ezrin strongly influenced the protein's secondary structure. Thus, we analyzed the protein structures of both cPC2wt and cPC2S812D by CD spectroscopy, which is a rather straightforward and simple method. Our results clearly demonstrate that the point mutation does not significantly affect the secondary structure elements of the protein. Both proteins show an identical $36 \% \alpha$-helical-, $13 \% \beta$-strand-, $16 \%$ turnand $35 \%$ random coil-content. Small alterations of the molecular structure however, cannot be resolved by this method. A detailed NMR analysis of the respective residues would be required, which can show slight differences in the molecular structure even though the CD spectra are basically the same. ${ }^{37}$

The quantification of the CPC2-PIGEA14 interaction revealed that the dissociation constants are in the ten nanomolar range, which is characteristic for a specific and biologically relevant PC2/PIGEA14 interaction. Of note, the affinity constant, i.e. the binding affinity of the PIGEA14-cPC2wt interaction is two times larger than that of the PIGEA14-pseudophosphorylated cPC2 (cPC2S812D) interaction (Table 1). The rate constants of association and dissociation are only slightly altered (Table 1) by the phosphorylation and are in the range of $10^{4}-10^{5} \mathrm{M}^{-1} \cdot \mathrm{s}^{-1}$ and $10^{-3} \mathrm{~s}^{-1}$, respectively. While Brownian motion would result in association/dissociation rates in the range of $10^{9}-10^{10} \mathrm{M}^{-1} \cdot \mathrm{s}^{-1}$, typical association rate constants for protein interactions are in the order of $10^{5}-10^{6} \mathrm{M}^{-1} \cdot \mathrm{s}$ ${ }^{1}$ as a result of the activation energy of protein complex formation. Dissociation rate constants vary within a wide range of $10^{-6}-10^{-1} \mathrm{~s}^{-1} .38,39$ The fact that our determined association rate constants were slightly lower compared to the average range of biological relevant interactions can be explained in manifold ways. First, in a cellular system association rates can be enhanced by alterations of the local $\mathrm{pH}$, ionic strength or other surroundings. Second, the protein complex exhibits a relatively small dissociation rate constant, which accounts for a tight binding and a strong binding affinity. 
The obtained results are discussed in light of the PC2 transport mechanism in the cell. Köttgen et al. ${ }^{20}$ investigated the interaction of cPC2wt and its mutants PC2S812D and PC2S812A with PACS-1 and PACS-2. By means of co-immunoprecipitation, they determined that binding of the C-terminal PC2 (Arg ${ }^{741}-\mathrm{Arg}^{871}$ ) to PACS-1 and PACS-2, which is mediated by an acidic amino acid cluster $\left(\mathrm{Asp}^{790}-\mathrm{Ser}^{820}\right)$ is increased upon PC2 phosphorylation at Ser ${ }^{812}$. In turn, replacing Ser812 by Ala greatly diminished these interactions. Later on, Fu et al. ${ }^{40}$ confirmed the finding that a PC2S812A mutation inhibits the interaction with PACS proteins. Using zebrafish larvea as a model organism, they discovered that the PC2S812A mutant rescued cyst formation being able to reach other subcellular compartments, whereas PC2S812D remained located at the ER via binding to PACS-2. Based on these results, Köttgen et al. ${ }^{17}$ developed a model of PC2 trafficking including the PC2 interaction partners PACS-1 and -2 as well as PIGEA14 (Fig. 7). This model implies that phosphorylated PC2 binds with high affinity to PACS-2 and thus, remains located at the ER. After dephosphorylation of PC2 at position Ser812 by protein phosphatase 2A (PP2A), PC2 is released by PACS-2 as the binding affinity is decreased, while the binding affinity to PIGEA14 is increased as demonstrated by our results. This change in binding affinities to two different proteins as a result of PC2phosphorylation can induce the transport of the protein complex to the Golgi network. However, another aspect should also be considered. Supported by the observations of Hidaka et al. ${ }^{17}$ and Celic et al. ${ }^{8}$, it is conceivable that the PC2 interface required for the interaction with PIGEA14 is blocked, when PC2 is in complex with PACS-2, which might additionally alter the binding affinity of PIGEA14 to PC2 in vivo. It has been shown that the amino acid sequence Gly833-Ser923 of cPC2 is sufficient to interact with PIGEA $14^{17}$ and that this proposed coiled-coil region is in close proximity to the domain responsible for the PC2/PACS interaction. \{Celic $2008 \# 100\}$ 


\subsection{Co-workers}

Schatz, Michaela; Hubrich, Raphael; Hoffmeister, Hellen; Witzgall, Ralph 


\subsection{References}

[1] Harris, P. C., and Torres, V. E. (2009) Polycystic kidney disease. Annu. Rev. Med. $60,321-337$.

[2] Koptides, M., and Deltas, C. C. (2000) Autosomal dominant polycystic kidney disease: molecular genetics and molecular pathogenesis. Hum. Genet. 107, 115-126.

[3] The International Polycystic Kidney Disease Consortium* (1995) Polycystic kidney disease: the complete structure of the PKD1 gene and its protein. Cell 81, 289-298.

[4] Cai, Y., Maeda, Y., Cedzich, A., Torres, V. E., Wu, G., Hayashi, T., Mochizuki, T., Park, J. H., Witzgall, R., and Somlo, S. (1999) Identification and characterization of polycystin-2, the PKD2 gene product. J. Biol. Chem. 274, 28557-28565.

[5] Mochizuki, T., Wu, G., Hayashi, T., Xenophontos, S. L., Veldhuisen, B., Saris, J. J., Reynolds, D. M., Cai, Y., Gabow, P. A., Pierides, A., Kimberling, W. J., Breuning, M. H., Deltas, C. C., Peters, D. J., and Somlo, S. (1996) PKD2, a gene for polycystic kidney disease that encodes an integral membrane protein. Science 272, 1339_ 1342.

[6] Giamarchi, A., Padilla, F., Coste, B., Raoux, M., Crest, M., Honoré, E., and Del mas, P. (2006) The versatile nature of the calcium-permeable cation channel TRPP2. EMBO Rep. 7, 787-793.

[7] Petri, E. T., Celic, A., Kennedy, S. D., Ehrlich, B. E., Boggon, T. J., and Hodsdon, M. E. (2010) Structure of the EF-hand domain of polycystin-2 suggests a mechanism for Ca2+-dependent regulation of polycystin-2 channel activity. Proc. Natl. Acad. Sci. U.S.A 107, 9176-9181. 
[8] Celić, A., Petri, E. T., Demeler, B., Ehrlich, B. E., and Boggon, T. J. (2008) Domain mapping of the polycystin-2 C-terminal tail using de novo molecular modeling and biophysical analysis. J. Biol. Chem. 283, 28305-28312.

[9] Streets, A. J., Needham, A. J., Gill, S. K., and Ong, A. C. M. (2010) Protein kinase D-mediated phosphorylation of polycystin-2 (TRPP2) is essential for its effects on cell growth and calcium channel activity. Mol. Biol. Cell. 21, 3853-3865.

[10] Zhou, J. (2009) Polycystins and primary cilia: primers for cell cycle progression. Annu. Rev. Physiol. 71, 83-113.

[11] Nauli, S. M., Alenghat, F. J., Luo, Y., Williams, E., Vassilev, P., Li, X., Elia, A. E. H., Lu, W., Brown, E. M., Quinn, S. J., Ingber, D. E., and Zhou, J.(2001) Polycystins 1 and 2 mediate mechanosensation in the primary cilium of kidney cells. Nat. Genet. 33, 129-137.

[12] Hsu, Y.-J., Hoenderop, J. G. J., and Bindels, R. J. M. (2007) TRP channels in kidney disease. Biochim. Biophys. Acta 1772, 928-936.

[13] Stayner, C., and Zhou, J. (2001) Polycystin channels and kidney disease. Trends Pharmacol. Sci. 22, 543-546.

[14] Tsiokas, L., Kim, S., and Ong, E.-C. (2007) Cell biology of polycystin-2. Cell. Signal. 19, 444-453.

[15] Tsiokas, L. (2009) Function and regulation of TRPP2 at the plasma membrane. Am. J. Physiol. Renal Physiol. 297, F1-9.

[16] Newby, L. J., Streets, A. J., Zhao, Y., Harris, P. C., Ward, C. J., and Ong, A. C. M. (2002) Identification, characterization, and localization of a novel kidney polycystin-1-polycystin-2 complex. J. Biol. Chem. 277, 20763-20773.

[17] Köttgen, M., and Walz, G. (2005) Subcellular localization and trafficking of polycystins. Pflugers Arch - Eur. J. Physiol. 451, 286-293.

[18] Witzgall, R. (2005) Polycystin-2--an intracellular or plasma membrane channel? Naunyn Schmiedebergs Arch. Pharmacol. 371, 342-347. 
[19] Hanaoka, K., Qian, F., Boletta, A., Bhunia, A. K., Piontek, K., Tsiokas, L., Sukhatme, V. P., Guggino, W. B., and Germino, G. G. (2000) Co-assembly of polycystin-1 and -2 produces unique cation-permeable currents. Nature 408, 990-994.

[20] Köttgen, M., Benzing, T., Simmen, T., Tauber, R., Buchholz, B., Feliciangeli, S., Huber, T. B., Schermer, B., Kramer-Zucker, A., Höpker, K., Simmen, K. C., Tschucke, C. C., Sandford, R., Kim, E., Thomas, G., and Walz, G. (2005) Trafficking of TRPP2 by PACS proteins represents a novel mechanism of ion channel regulation. EMBO J. 24, 705-716.

[21] Hidaka, S., Könecke, V., Osten, L., and Witzgall, R. (2004) PIGEA-14, a novel coiled-coil protein affecting the intracellular distribution of polycystin-2. J. Biol. Chem. 279, 35009-35016.

[22] Witzgall, R. (2005) New developments in the field of cystic kidney diseases. Curr. Mol. Med. 5, 455-465.

[23] Mokhtarzada, S., Yu, C., Brickenden, A., and Choy, W.-Y. (2011) Structural characterization of partially disordered human Chibby: insights into its function in the Wnt-signaling pathway. Biochemistry 50, 715-726.

[24] Mofunanya, A., Li, F.-Q., Hsieh, J.-C., and Takemaru, K.-I. (2009) Chibby forms a homodimer through a heptad repeat of leucine residues in its $C$-terminal coiledcoil motif. BMC Mol. Biol. 10, 41.

[25] Behn, D., Bosk, S., Hoffmeister, H., Janshoff, A., Witzgall, R., and Steinem, C. (2010) Quantifying the interaction of the C-terminal regions of polycystin-2 and polycystin-1 attached to a lipid bilayer by means of QCM. Biophys. Chem. 150, 47-53.

[26] Wegener, J., Janshoff, A., and Steinem, C. (2001) The quartz crystal microbalance as a novel means to study cell-substrate interactions in situ. Cell Biochem. Biophys. 34, 121-151. 
[27] Cooper, M. A., and Singleton, V. T. (2007) A survey of the 2001 to 2005 quartz crystal microbalance biosensor literature: applications of acoustic physics to the analysis of biomolecular interactions. J. Mol. Recognit. 20, 154-184.

[28] Schein, C. H., and Noteborn, M. H. M. (1988) Formation of soluble recombinant proteins in Escherichia coli is favored by lower growth temperature. Nat. Biotechnol. 6, 291-294.

[29] Rudolph, R., and Lilie, H. (1996) In vitro folding of inclusion body proteins. FA SEB J. 10, 49-56.

[30] Hochuli, E., Döbeli, H., and Schacher, A. (1987) New metal chelate adsorbent selective for proteins and peptides containing neighbouring histidine residues. $J$. Chromatogr. 411, 177-184.

[31] Schumann, F., Hoffmeister, H., Bader, R., Schmidt, M., Witzgall, R., and Kalbitz er, H. R. (2009) Ca2+-dependent conformational changes in a C-terminal cytosolic domain of polycystin-2. J. Biol. Chem. 284, 24372-24383.

[32] Yao, X., Kwan, H.-Y., and Huang, Y. (2005) Regulation of TRP channels by phosphorylation. Neurosignals 14, 273-280.

[33] Marks, F. (1996) Protein phosphorylation, VCH, Weinheim, New York.

[34] Cai, Y., Anyatonwu, G., Okuhara, D., Lee, K.-B., Yu, Z., Onoe, T., Mei, C.-L., Qian, Q., Geng, L., Wiztgall, R., Ehrlich, B. E., and Somlo, S. (2004) Calcium dependence of polycystin-2 channel activity is modulated by phosphorylation at Ser812. J. Biol. Chem. 279, 19987-19995.

[35] Ben-Tal, N., Honig, B., Bagdassarian, C. K., and Ben-Shaul, A. (2000) Association entropy in adsorption processes. Biophys. J. 79, 1180-1187.

[36] Bosk, S., Braunger, J. A., Gerke, V., and Steinem, C. (2011) Activation of F-actin binding capacity of ezrin: synergism of $\mathrm{PIP}_{2}$ interaction and phosphorylation. Biophys. J. 100, 1708-1717.

[37] Fischer, D., Mukrasch, M. D., Biernat, J., Bibow, S., Blackledge, M., Griesinger, C., Mandelkow, E., and Zweckstetter, M. (2009) Conformational changes specific 
for pseudophosphorylation at serine 262 selectively impair binding of tau to microtubules. Biochemistry 48, 10047-10055.

[38] Schreiber, G. (2002) Kinetic studies of protein-protein interactions. Curr. Opin. Struct. Biol. 12, 41-47.

[39] Myszka, D. G. (1997) Kinetic analysis of macromolecular interactions using surface plasmon resonance biosensors. Curr. Opin. Biotechnol. 8, 50-57.

[40] Fu, X., Wang, Y., Schetle, N., Gao, H., Pütz, M., Gersdorff, G. von, Walz, G., and Kramer-Zucker, A. G. (2008) The subcellular localization of TRPP2 modulates its function. J. Am. Soc. Nephrol 19, 1342-1351. 
7

Directing the Adsorption of Filamentous Actin Within Surface-modified, Cylindrical Nanopores

\subsection{Abstract}

Ezrin is a member of the ERM (ezrin radixin moesin) family that acts as a dynamic linker between the plasma membrane and the actin cytoskeleton. Hence, it is involved in membrane organization, dynamics and determination of the cellular shape. Ezrin is highly enriched in microvilli of polarized epithelial cells, where it binds F-actin with a C-terminal binding side upon activation via $N$-terminal PIP2 (L- $\alpha$-phosphatidylinositol4,5-bisphosphate) binding and phosphorylation at $\mathrm{Thr}^{567}$. Highly ordered nanoporous aluminum oxide (AAO) films provide similar dimension as the microvilli. Owing to their optical transparency, they are versatile microvilli mimics to investigate the interaction of ezrin with F-actin by means of optical waveguide spectroscopy (OWS) and confocal laser scanning microscopy (CLSM).

Here, we developed three model systems based on nanoporous AAO films that enabled us to direct and control F-actin adsorption within and atop the porous surface. It was further aimed to study the influence of actin bundles inside the pores on the mechanical properties of the F-actin network atop the pores by means of force spectroscopy. 


\subsection{Introduction}

As the structural framework of eukaryotic cells, the cytoskeleton is not only responsible for maintaining the shape and structure of the cell, but also controls dynamic processes, provides cellular motility and spatially organizes cellular contents. Although a large number of proteins contribute to the cytoskeletal organization, it is mainly composed of three types of protein filaments: actin filaments, intermediate filaments and microtubules. ${ }^{1}$

Microtubules build up a hollow cylinders (length, $l=25 \mu \mathrm{m}$, diameter, $d=25 \mathrm{~nm}$ ) as a result of polymerization of $\alpha$ - and $\beta$-tubulin heterodimers. The main role of microtubules is the regulation of intracellular traffic and genome segregation during mitosis. Tetrameric intermediate filaments $(d=10 \mathrm{~nm})$ are not directly involved in cellular dynamics, but play a structural role, providing the stability and mechanical strength of the cell. ${ }^{2,1}$

Actin filaments are semi-flexible polymers with a mean diameter of $7 \mathrm{~nm}$ and a variable length ranging from 0.1-20 $\mu \mathrm{m} .{ }^{3}$ Filamentous-actin (F-actin) is composed of globular actin (G-actin) protein, which consists of 375 amino acids and has a molecular weight of $M=43 \mathrm{kDa}($ Fig. 7.1$) .^{4}$ 


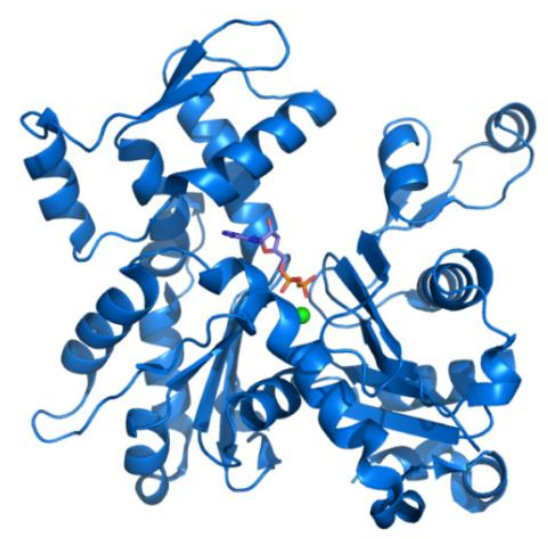

Fig. 7.1 Ribbon diagram of G-actin (PDB code 1j6z) in complex with a $\mathrm{Mg}^{2+}$ ion (green) and an ATP molecule (orange).

Every G-actin monomer harbors an ATP and $\mathrm{Mg}^{2+}$ binding site and polymerizes via head-to-tail interaction into filaments with a distinct polarity. Vertebrates contain six isoforms of actin: four muscular $\alpha$-forms and two non-muscular forms ( $\beta$ and $\gamma$ ). ${ }^{5,6}$ On basis of the crystal structure of G-actin that had been solved in 1990 by Kabsch et al. , the atomic structure of F-actin was determined by Holmes et al... ${ }^{8}$ They stated that the filament has a helical structure with each monomer rotated by $166^{\circ}$ and that it morphologically appears as two right handed helices.

The molecular mechanism of actin polymerization can be divided in three steps including nucleation, growth phase and steady state (Fig. 7.2). 


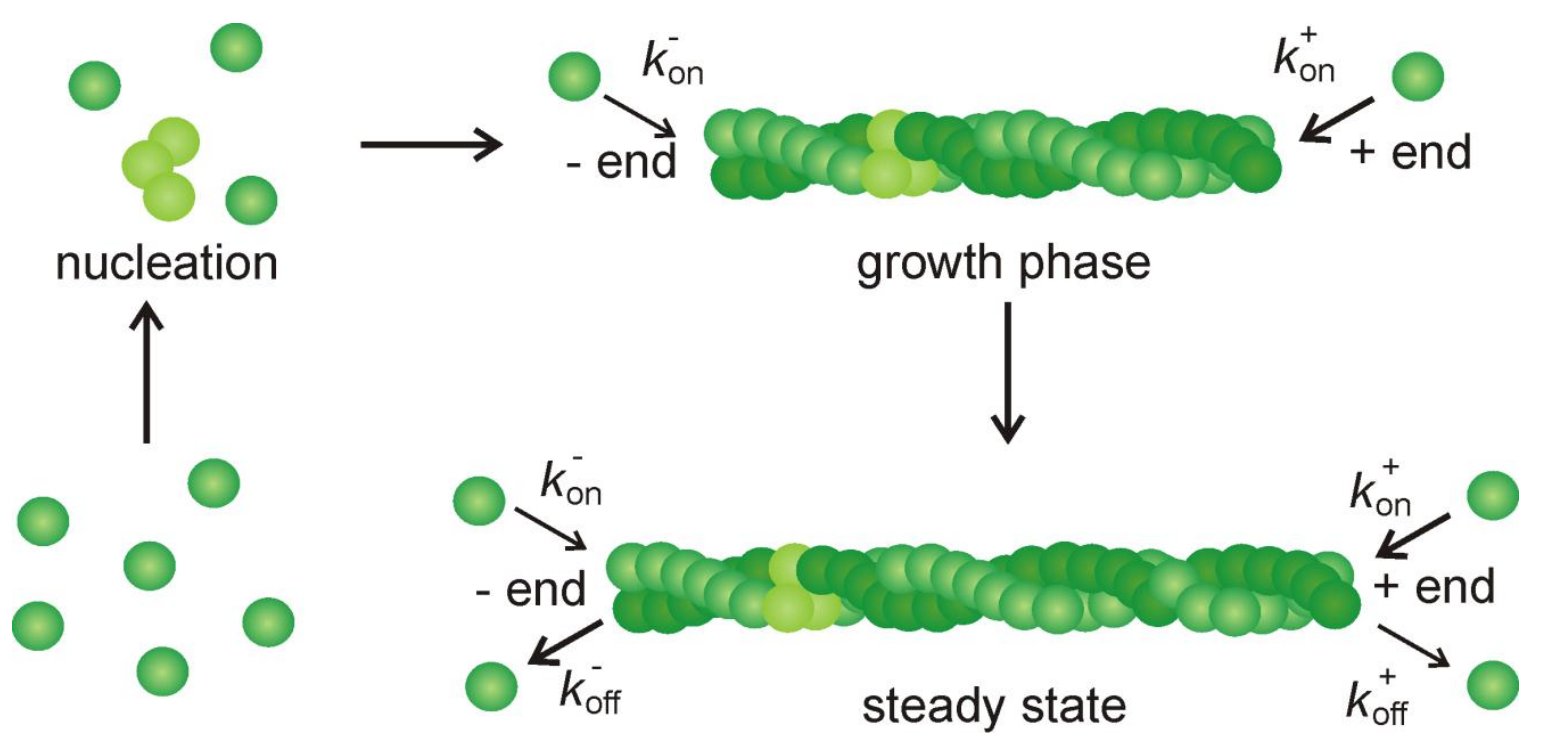

Fig. 7.2 Molecular mechanism of actin polymerization. Nucleation leads to the formation of a nucleitrimer (light green), elongation during the growth phase of the polarized filament results in a steady state with a constant filament length.

Formation of the nuclei is the time limiting step of the polymerization process; the following polymer growth includes the addition of ATP-bound actin. Due to the filament polarization, monomer addition occurs significantly faster at the plus end than at the minus end $\left(k_{\mathrm{on}^{+}}{ }^{-} k_{\mathrm{on}}{ }^{-}\right)$. Within the filament the ATP gets slowly hydrolyzed and stable $\mathrm{ADP}$-actin is formed. At the steady state, the rate of actin polymerization equals the rate of depolymerization (treadmilling). As ADP-actin depolymerizes more readily than ATP-actin, the rate of actin loss is higher at the minus end than at the plus end $\left(k_{\text {off }}>k_{\text {off }}{ }^{+}\right)$, which is capped by newly added ATP-actin monomers. The concentration of G-actin at this steady state is called critical concentration $\left(c_{c}\right)$. Above that concentration, actin polymerizes, below it, filaments will depolymerize. ${ }^{9,10}$

In vitro, actin polymerization can be induced by the addition of $\mathrm{Mg}^{2+}$ and $\mathrm{K}^{+}$, which reduce the critical concentration needed for polymerization by the factor of hundred to approximately $c_{c}=7 \mu \mathrm{M}$ at $4{ }^{\circ} \mathrm{C}$. Raising the temperature, $c_{c}$ can be further decreased. ${ }^{11}$ In vivo however, the interplay of a variety of actin binding proteins (ABPs) governs the turnover of actin polymerization (Fig. 7.3). 

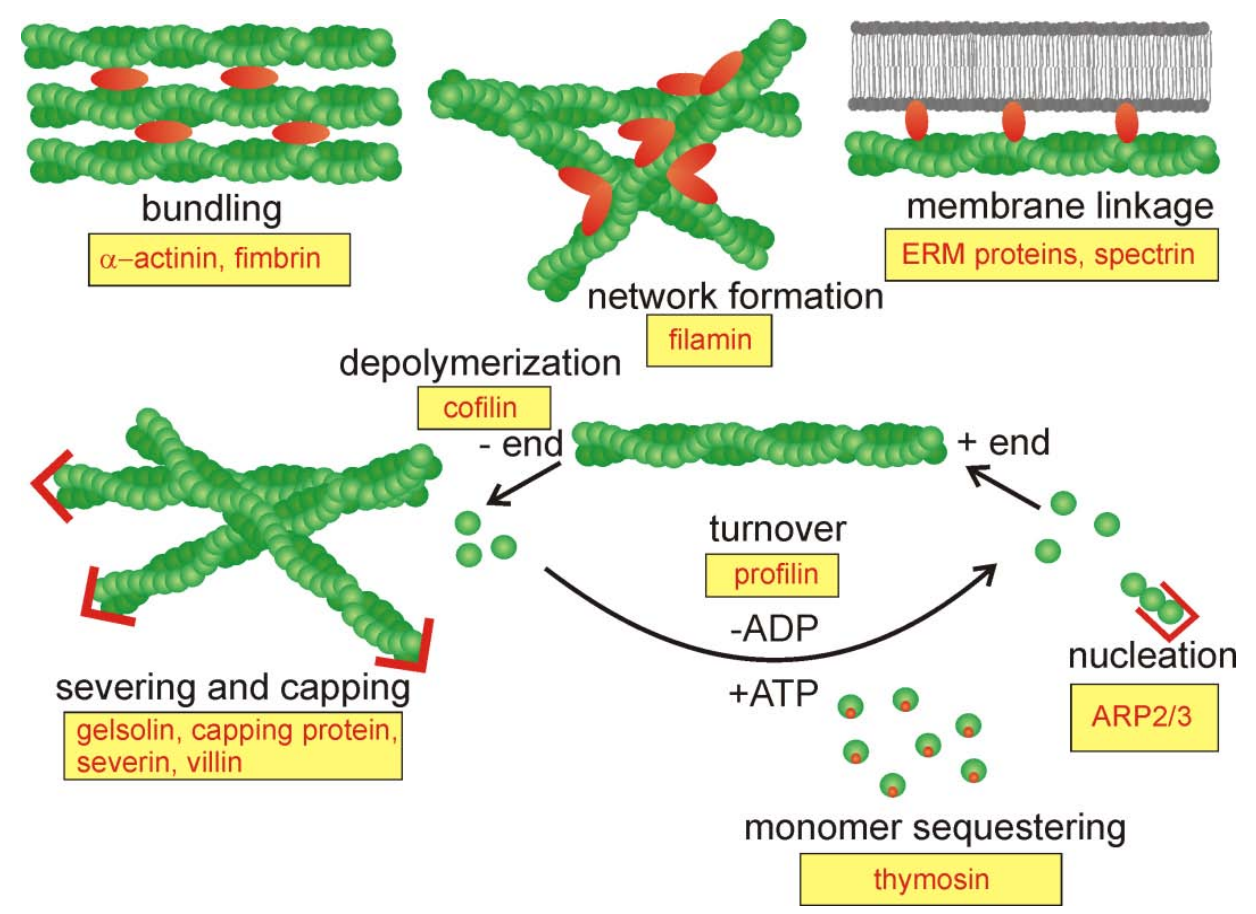

Fig. 7.3 Schematic drawing of a selection of actin binding proteins (ABPs, red) and their specific function.

Those ABPs either sequester G-actin monomers, facilitate nuclei formation or serve as capping proteins on either end of the polymer. Other ABPs connect the filaments with each other and facilitate the formation of networks (i.e. filamin) or bundles (i.e. $\alpha$ actinin). Only by forming three dimensional, flexible networks, the dynamic function of cytoskeletal F-actin can be guaranteed..$^{12-14}$

Certain actin binding proteins are capable of linking F-actin filaments to the plasma membrane. Members of the ezrin radixin moesin (ERM) protein family ${ }^{15-17}$ for instance are structured in a way that intramolecular interaction between the $\mathrm{N}$ - and $\mathrm{C}$-terminal regions mask their protein interaction site and keep the proteins in an inactive state (Fig. 7.4 B). ${ }^{18,19}$ ERM proteins unfold and become activated in response to protein phosphorylation and binding to the L- $\alpha$-phosphatidylinositol-4,5-bisphosphate $\left(\mathrm{PIP}_{2}\right)$ receptor lipid anchored in the plasma membrane. ${ }^{20,21}$ Upon activation ERM proteins function as dynamic membrane-cytoskeleton crosslinkers. ${ }^{22}$ Structurally ERM proteins can be divided in a 300 amino acids containing, $N$-terminal band four point one, ezrin, radix- 
in, moesin (FERM) domain, which is responsible for PIP2-binding 23,24 , an $\alpha$-helical linker region and the $C$-terminal ERM association domain (C-ERMAD), which harbors an Factin binding site (Fig. 7.4 A). ${ }^{25}$

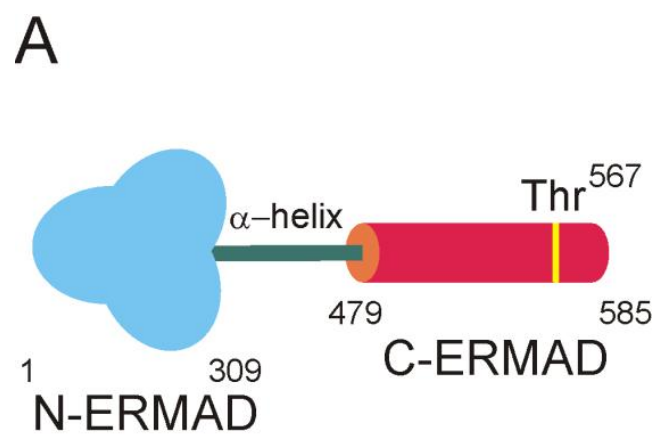

B

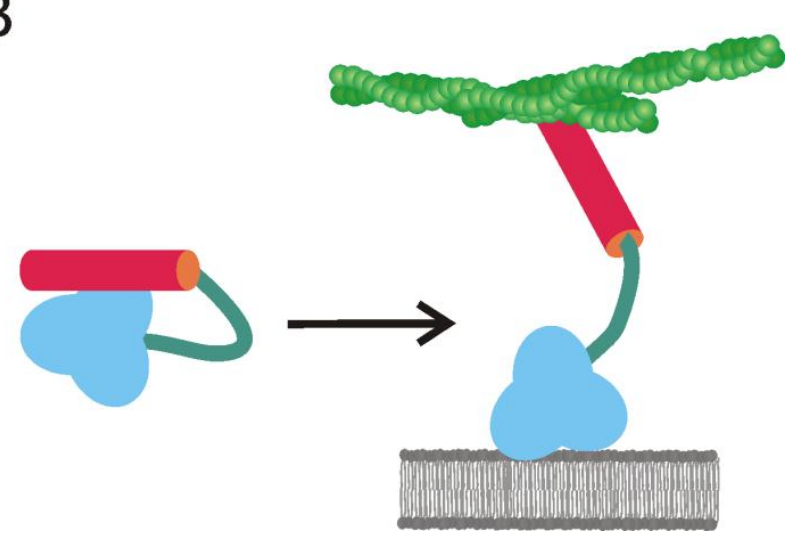

Fig. 7.4 (A) Topology of the ezrin domain, including the PIP2 binding site (blue, N-ERMAD), $\alpha$-helical linker region (green) and the C-ERMAD (red) that harbors the F-actin binding site (551-585) and a phosphorylation site (yellow).(B) Model of ezrin that binds F-actin with its C-ERMAD after being activated via $\mathrm{PIP}_{2}$ binding and phosphorylation at $\mathrm{Thr}^{567}$.

Whereas moesin is mainly expressed in a wide range of endothelial cells and radixin is enriched in adherent junctions, ezrin was identified as a F-actin linker protein in microvilli of intestinal epithelial cells and membrane ruffles. ${ }^{15,26}$ Inside these finger-like structures, ezrin links bundles of F-actin to the plasma membrane and thus provides maintaining the shape of these cellular protrusions (Fig. 7.5 A). 


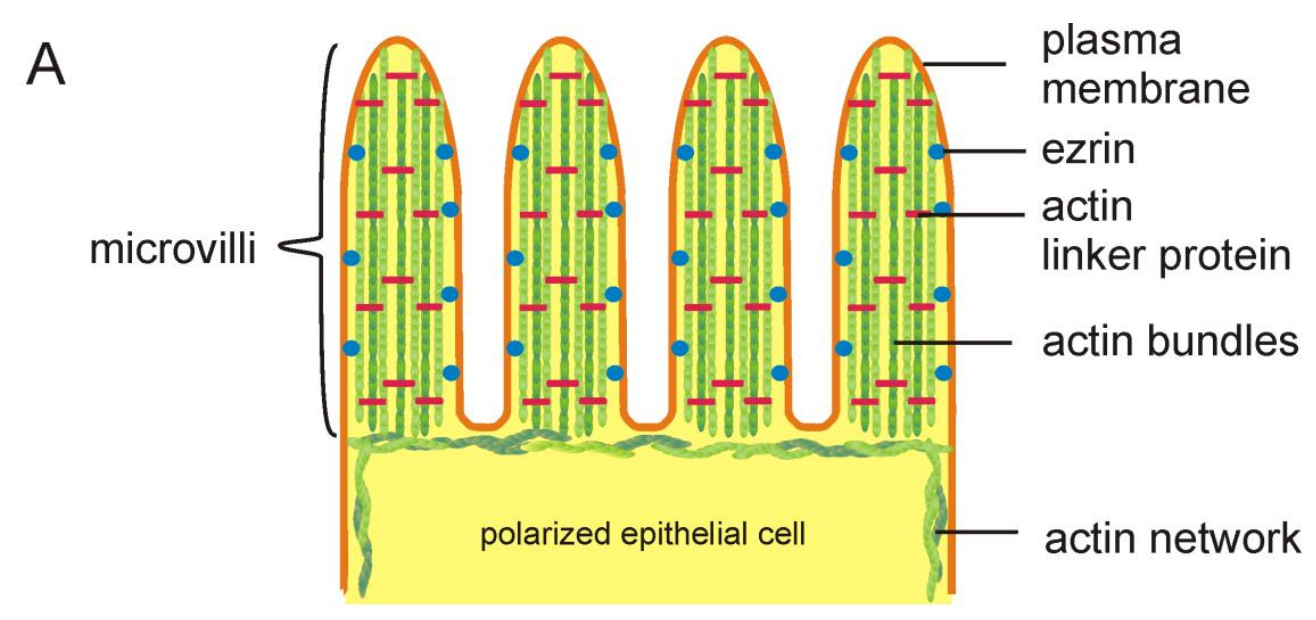

B

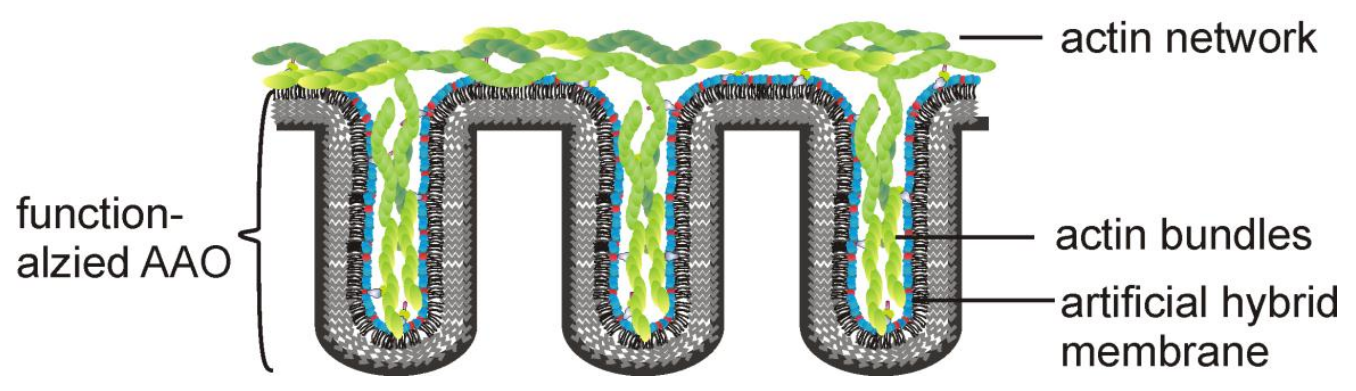

Fig. 7.5 (A) Schematic drawing of a polarized epithelial cell. Within the microvilli $(d \approx 80-150 \mathrm{~nm}$, $l \approx 1-1.7 \mu \mathrm{m}$ ) bundled F-actin (green) is attached to the plasma membrane via ezrin (blue) linkage. (B) AAO set up $(d \approx 25-80 \mathrm{~nm}, l \approx 3.5-7 \mu \mathrm{m})$ that mimics the microvilli with solid a supported hybrid membrane, actin bundles inside the pores and a F-actin network atop the pores.

In this work, we raised the question of how the mechanical properties of the cellular Factin network are influenced by F-actin bundles inside of the microvilli. To answer this question, first, a proper model system was be chosen that structurally correlates with the microvillar geometry. This model system was then be designed in a way that it mimics microvilli and provides a platform to monitor and verify the adsorption processes of all involved molecules (Fig. $7.5 \mathrm{~B}$ ).

Owing to their optical transparency and tunable geometry, nanoporous anodic aluminum oxide (AAO) substrates can be utilized to mimic cellular microvilli as AAO films have similar dimensions (microvilli dimensions: $d \approx 80-150 \mathrm{~nm}, l \approx 1-1.7 \mu \mathrm{m}$ ). ${ }^{27}$ Recently, nanoporous AAO substrates have been introduced by Lazzara et al., as a biosensor 
platform that allows to follow the adsorption of macromolecules inside the porous area in real time. ${ }^{28}$ In 2012, modified phospholipids monolayers that line the porous regions were used to allow specific coupling of proteins. ${ }^{29}$ Based on these developments, a model system will be designed that allows the layer by layer assembly of all components needed, to mimic F-actin linkage atop and inside of the microvilli. To elucidate the impact of actin bundles inside the pore interior on the mechanical properties of the overlying F-actin network, force spectroscopy experiments will be performed. Thus, it was aimed to create two setups: one, where the F-actin network is adsorbed only atop the AAO pores and another, where F-actin bundles are adsorbed inside the porous area below the overlying network.

\subsection{Results}

\subsubsection{Design of a Model System}

To direct the adsorption of filamentous actin (F-actin) within anodic aluminum oxide (AAO) nanopores, different functionalization strategies were developed. These routes are depicted in Fig. 7.6. With the first route (Fig. 7.6, 1) F-actin immobilization was achieved via electrostatic interaction using the positively charged silane aminopropyltriethoxysilane (APTES). It was aimed to control, whether F-actin adsorption takes place only atop or also within the pores, was carried out by choosing AAO substrates with different pore diameters (see Fig. 7.7). It was expected that using smaller pore sizes with pore diameters of $d \approx 25 \mathrm{~nm}$, F-actin with a mean diameter of $d=7 \mathrm{~nm}$ is exclusively found atop the AAO pores. However, at larger pore diameters, it was assumed that F-actin would penetrate the pore interior. The second route (Fig. 7.6, 2) was designed to offer a lateral mobile surface composed of a solid supported hybrid membrane to electrostatically immobilize F-actin. Electrostatic interaction was facilitated by integrating the positively charged lipid DOEPC in a DOPC lipid matrix (see Table 3.1, 
page 32). Hence, this system allows to vary two parameter in order to direct F-actin adsorption, the DOEPC content and the pore diameter. The third route (Fig. 7.6, 3) was developed to mimic the nature of microvilli best possibly. Therefore, the protein ezrin that connects the plasma membrane of microvilli with the actin cytoskeleton, was immobilized on a solid supported hybrid membrane containing the natural ezrin linker lipid PIP2.

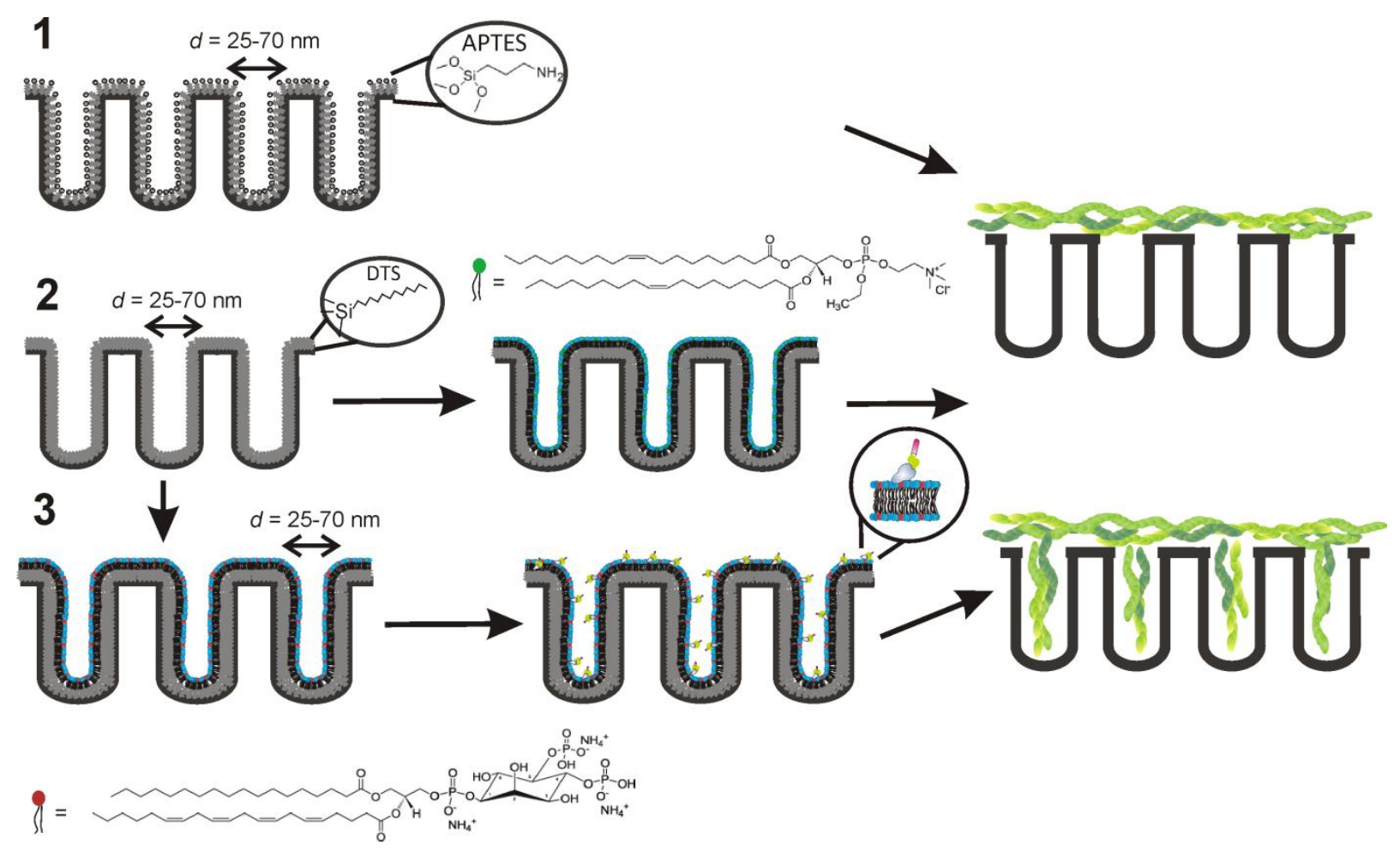

Fig. 7.6 Schematic drawing showing the different routes to direct F-actin adsorption within surface modified cylindrical nanopores. (1) APTES covered AAO with different pore diameters, (2) DOEPC containing membranes on DTS functionalized AAO and (3) ezrin bound to a $\mathrm{PIP}_{2}$ containing membrane on DTS functionalized AAO.

In all three cases, it was aimed to control F-actin polymerization by using AAO substrates with different pore sizes and a constant height of $h=7 \mu \mathrm{m}$. AAO films with of pore diameters ranging from $d=25-65 \mathrm{~nm}$ that were used in this work, are depicted in Fig. 7.7 as SEM micrographs. Owing to the optical transparency of AAO all adsorption 
processes (spreading of vesicles, ezrin, F-actin adsorption) were studied by means of confocal laser scanning microscope (CSLM) and optical waveguide spectroscope (OWS). After appropriate conditions to direct the F-actin adsorption according to the two states depicted in Fig. 7.6 (right hand side) were found, force spectroscopy experiments should be conducted to elucidate the influence of F-actin filaments inside the pore interior on the mechanical properties of the overlying F-actin network.
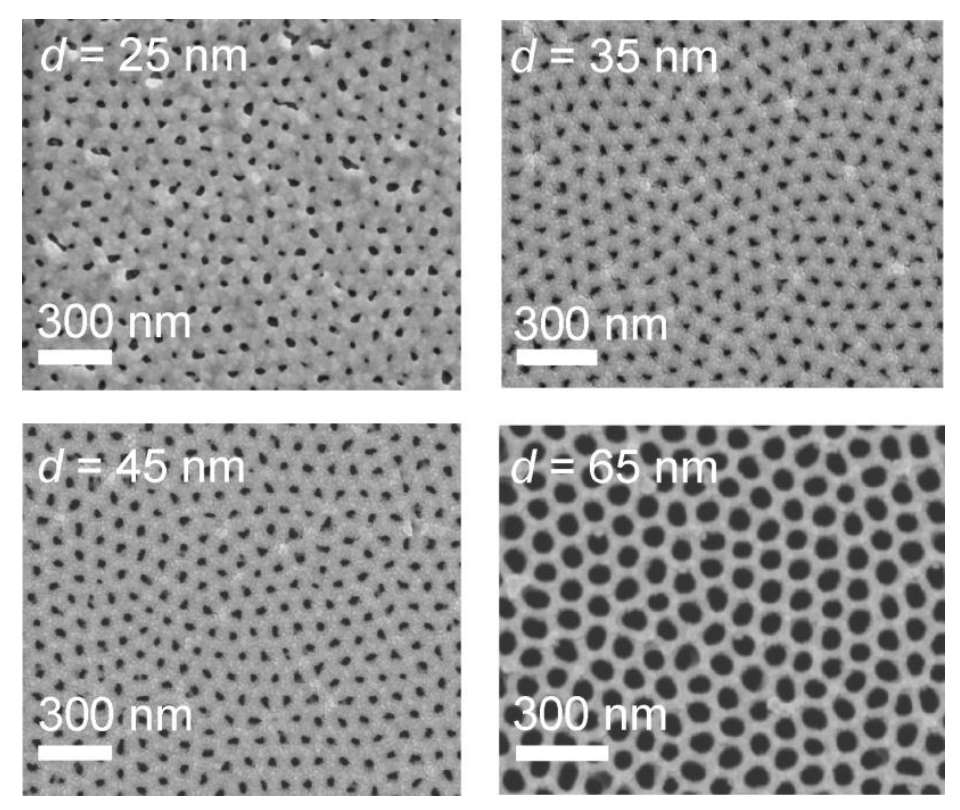

Fig. 7.7 SEM micrograph showing AAO substrates with different pore sizes.

\subsubsection{F-actin Adsorption Within Porous AAO Functionalized with APTES}

To direct F-actin adsorption within porous $\mathrm{AAO}$, first, a rather simple model system with only little control parameters was developed using silane chemistry. The positively charged aminopropyl-triethoxysilane (APTES) was used to functionalize the AAO surface as described in chapter 3.1.2 (page 29) and to immobilize F-actin via electrostatic interaction. Freshly polymerized muscular F-actin (see chapter 3.3.7, page 54, $\sim 0.7 \mu \mathrm{M}$ ) labeled with AlexaFluor488 phalloidin was added to APTES functionalized 
AAO substrates in F-buffer (50 mM KCl, $0.2 \mathrm{mM} \mathrm{MgCl}_{2}, 0.1 \mathrm{mM}$ EDTA, $0.1 \mathrm{mM} \mathrm{NaN}$, $20 \mathrm{mM}$ TRIS/HCl, $\mathrm{pH} 7.4$ ) and incubated overnight at $4{ }^{\circ} \mathrm{C}$. The AAO had diffrent pore diameters of $25 \mathrm{~nm}, 35 \mathrm{~nm}, 45 \mathrm{~nm}$ to $65 \mathrm{~nm}$. In Fig. $7.8 \mathrm{x}-\mathrm{y}$ plane fluorescence images are depicted (green fluorescence of the AlexaFluor488 phalloidin), showing the shape and structure of actin filaments on top of the AAO pores. Although all samples were prepared in the same manner, the filaments exhibit different structural features. In some cases long individual filaments that extend over several micrometers in height were visualized (Fig. 7.8 A), while mostly a carpet of short and densely packed F-actin was observed atop the AAO (Fig. 7.8 B).
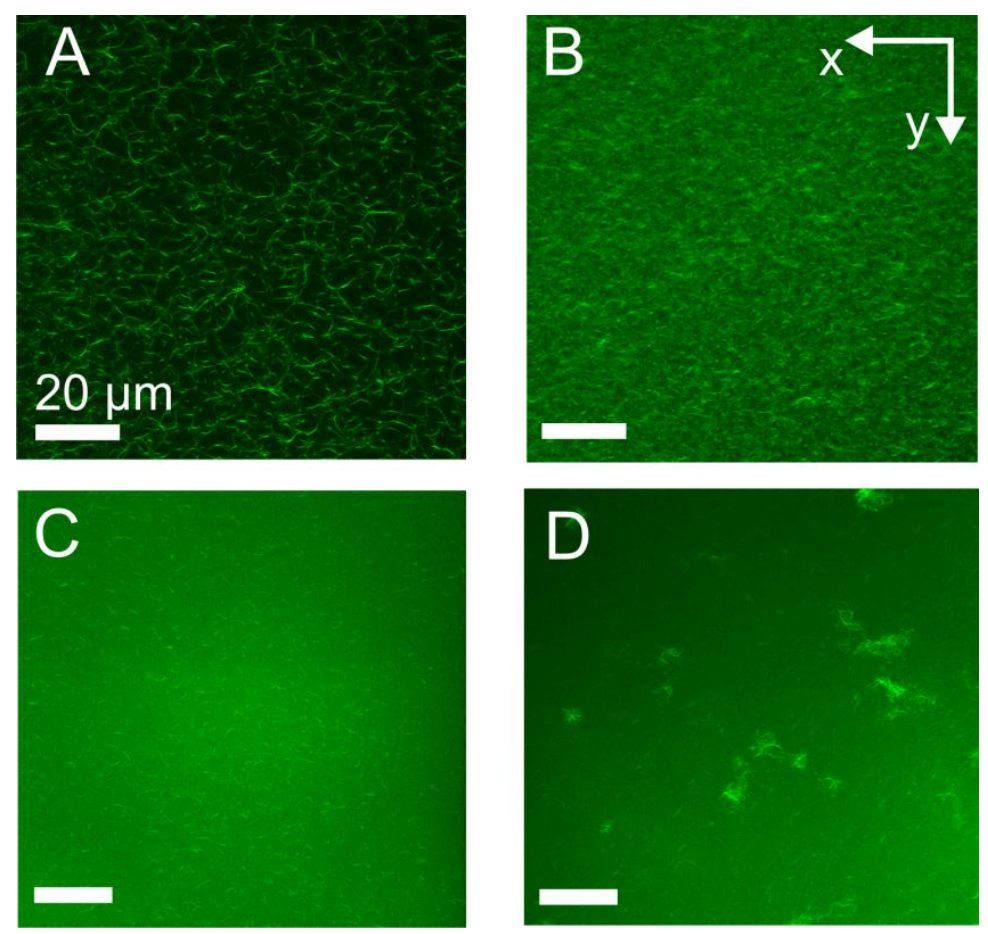

Fig. 7.8 Different exemplary fluorescence $x-y$ plane images of F-actin (labled with Alexa Fluor488 phalloidin) polymerized on and within AAO covered with APTES: (A) long individual filaments, (B) short filaments, (C) few filaments atop the AAO surface and (D) filamentous coils. The nature of F-actin was independent from the set pore diameter.

In some cases, only few individual filaments were observed (Fig. 7.8 C). The bright, green background fluorescence indicates that the fluorescence of filaments inside the 
pores seems to cover the one above the pores and thus leads to a reduced resolution of the filaments that were adsorbed atop the pores. This phenomenon was imaged more often, if F-actin was adsorbed on porous AAO that have larger pore diameters (>35 nm), a fact that shows the F-actin penetration dependence from the pore diameter. In very few cases, no individual filaments were visualized, but few large coil-like structures (Fig. 7.8 D). These experiments were interpreted as non successful, as they cause unexpected results regarding the pore penetration ability of actin in a way that no dependence of the F-actin penetration ability from the pore diameter could be found.

The F-actin content inside the AAO pores as a function of the pore diameter can be best visualized by means of cross section images ( $x-z$ plane images, $z$-lines, Fig. 7.9). Two limitting cases (A and B) of F-actin penetration are depicted for each pore size separately.
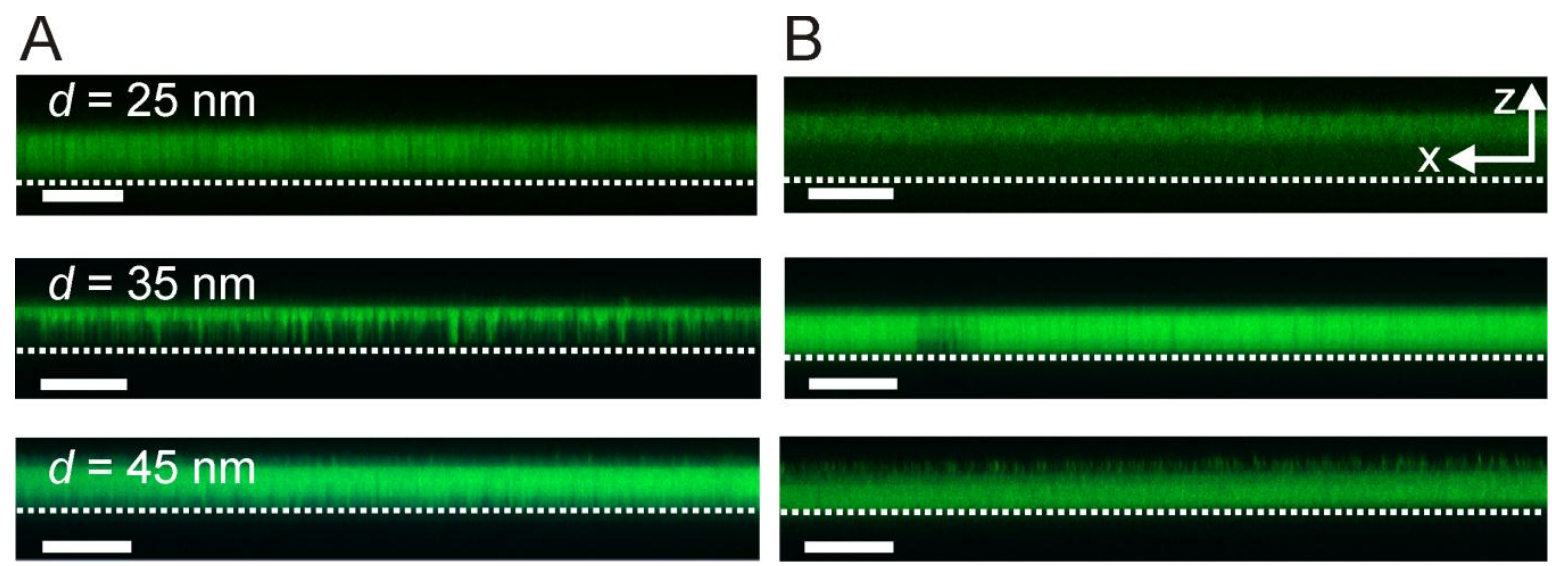

\section{$d=65 \mathrm{~nm}$}
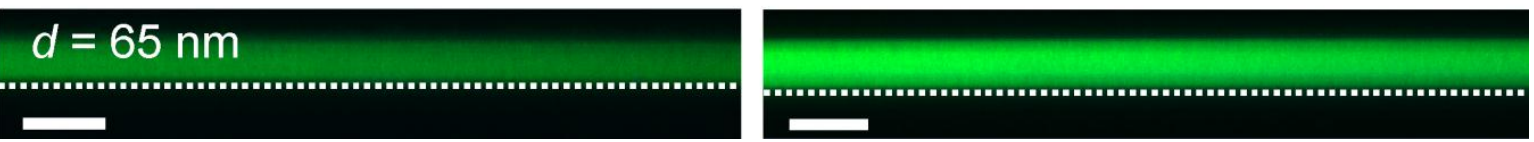

Fig. 7.9 Fluorescence $x-z$ plane images showing the two border cases (A and $\mathbf{B}$ ) of different F-actin (labled with Alexa Fluor488 phalloidin) content inside the APTES covered AAO pore interior for AAO pores with a variable diameters (AAO height, $h=7 \mu \mathrm{m}$ ). The dashed line represents the AAO pore bottom. Scale bar: $20 \mu \mathrm{m}$. 
In case of the AAO films with a pore diameter of $d=25 \mathrm{~nm}$, a green fluorescence is either visualized only atop the pores (Fig. 7.9 A) or partly inside them (Fig. 7.9 B; the AAO pore bottom is marked as a white, dashed line). It turned out that the propability of finding filaments inside the porous area is increased as a function of pore diameter. While for AAO pores with sizes of $d=35 \mathrm{~nm}$, there was still a possibility of partial filament exclusion from the pore interior (Fig. 7.9 A and B), AAOs with $d=45 \mathrm{~nm}$ were completely filled with F-actin (labled with Alexa Fluor488 phalloidin) in most cases. For AAO pores with a diameter of $d=65 \mathrm{~nm}$, the pore interior was entirely filled with actin filaments in all experiments, indicated by a homogeneously distributed green fluorescence. To prove that F-actin adsorption was achieved via specific, electrostatic binding at the positively charged APTES surface, a control experiment with non functionalized AAO was performed. In this case, no fluorescence of the Alexa Fluor488 phalloidin was visualized inside or atop the pores, demonstrating the absence of Factin. It was aimed to gain a higher control of F-actin exclusion from the AAO pore interior by selectively functionalizing the pore rims with APTES ${ }^{30}$, while the pore interior remined completely unfunctionalized (Fig. 7.10). Although it was expected, that filament penetration would be significantly reduced compared to the AAO substrates that were completely lined with APTES, the obtained results were identical to those, shown in Fig. 7.9. 


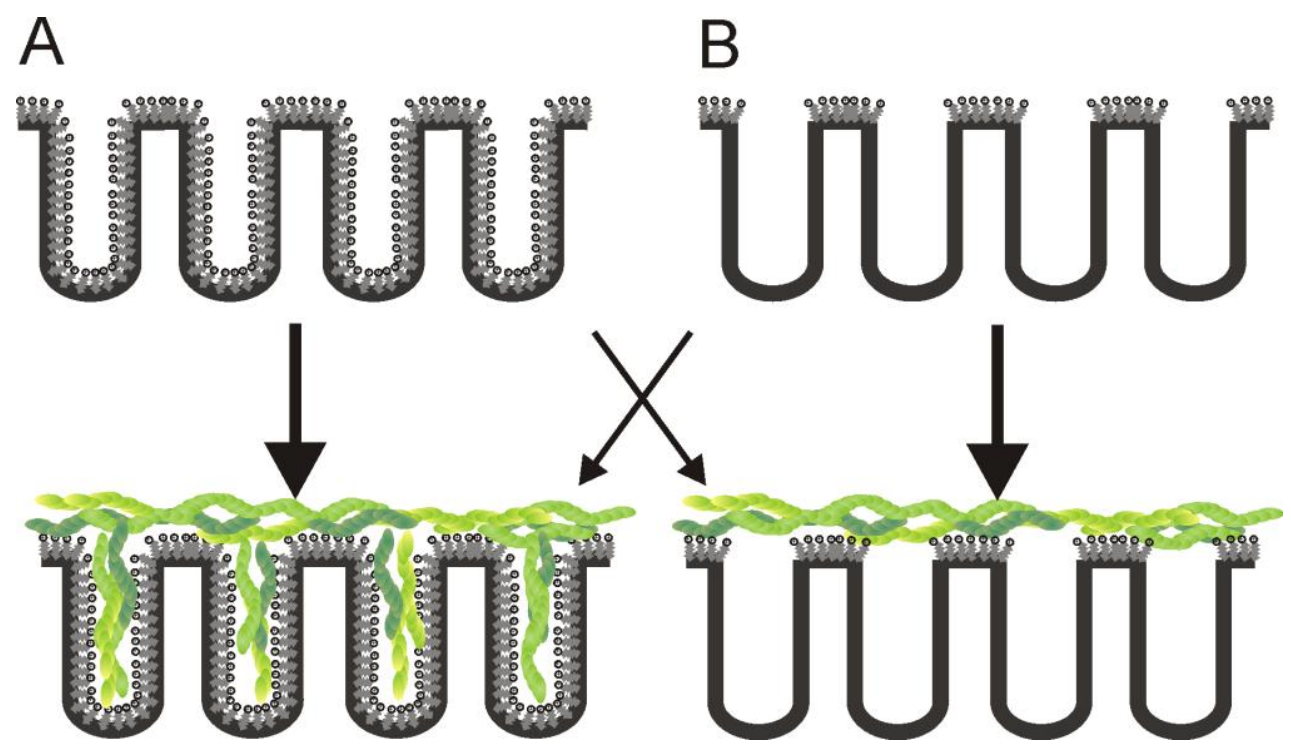

Fig. 7.10 Schematic drawing of porous AAO films that are either completely functionalized with APTES (A) or APTES only on the pore rims (B).

In conclusion, a correlation between F-actin penetration and pore diameter was found, but a complete exclusion of F-actin from the pore interior was not achieved in a reproducible manner. However, conditions needed to be developed, where the F-actin network exclusively binds atop the AAO pores, so that force spectroscopy experiments could be also performed with this system. Thus, it became obvious that directing the Factin adsorption only by varying the pore diameter was impossible and other control parameters had to be introduced.

\subsubsection{F-actin Adsorption Within Porous AAO with a DOEPC Containing Membrane}

\section{Confocal Laser Scanning Microscopy Experiments}

Since it had turned out that the pore diameter, as the only variable parameter to direct F-actin adsorption, was insufficient to create conditions that completely exclude actin filaments from the pore interior, a second variable parameter was implemented. This strategy (second route in Fig. 7.6.) includes F-actin adsorption on positively charged 
solid supported hybrid membranes with different surface charge densities. To obtain positively charged membranes different amounts of the positively charged DOEPC $(30-50 \mathrm{~mol} \%)$ were integrated in a DOPC matrix. The AAO pore diameters wereset to be $d=35$ and $45 \mathrm{~nm}$, so that the pores are small enough to potentially exclude F-actin from the interior, but large enough to allow for force spectroscopy experiments.

Fig. 7.11 shows a homogeneous, blue perylene fluorescence of a solid supported hybrid membrane composed of DOPC/DOEPC/perylene, 79:20:1 on an AAO surface that was functionalized with dodecyl-trichlorosilane (DTS). The homogenously distributed perylene fluorescence of the $\mathrm{x}-\mathrm{z}$ plane image (Fig. $7.11 \mathrm{~B}$ ) demonstrates that the membrane was lining the whole pore interior.
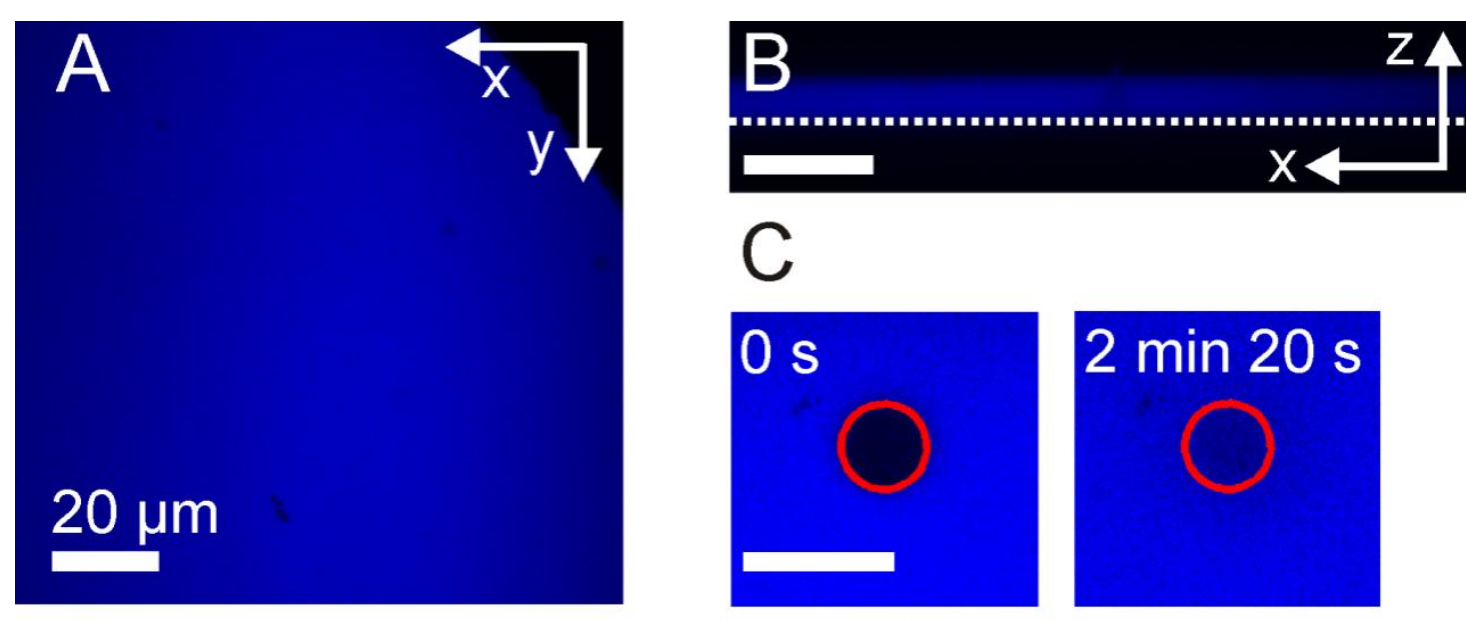

Fig. 7.11 Fluorescence $x-y$ plane (A) and $x-z$-plane (B) image of a membrane composed of DOPC/DOEPC/perylene, 79:20:1 on DTS functionalized AAO (AAO height, $h=7 \mu \mathrm{m}$ ). (C) Exemplary fluorescence images showing the time course of the recovery of fluorescence intensity after bleaching.

Fluorescence recovery after photobleaching (FRAP) experiments show the relatively slow recovery of fluorescence intensity after bleaching compared to solid supported planar membranes (Fig. 7.11 C). Nevertheless, the determined recovery time was in accordance with those obtained in the literature, where a POPC membrane that lined the pore interior of AAO $(d=75 \mathrm{~nm}, h=3.5 \mu \mathrm{m})$ were investigated by means of FRAP. ${ }^{29}$ 

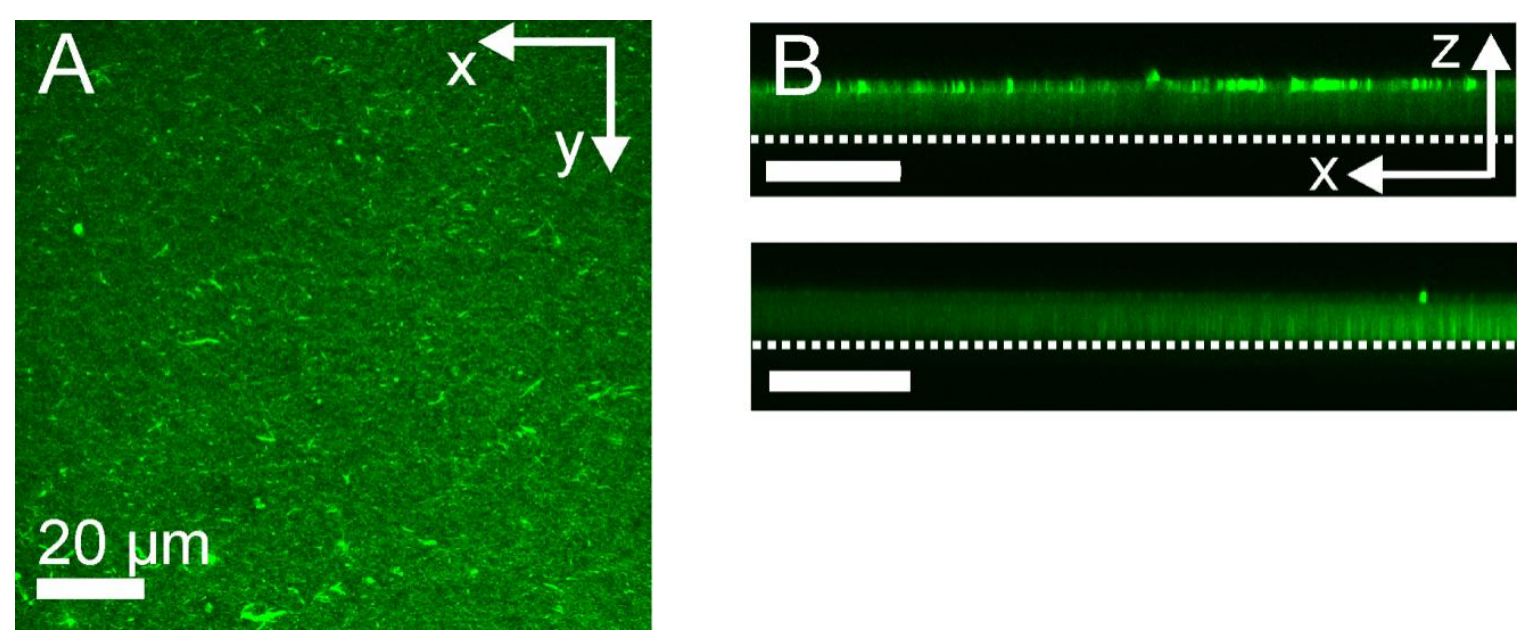

Fig. 7.12 Fluorescence x-y plane (A) and x-z-plane (B) image of AlexaFluor488 phalloidin labeled F-actin polymerized on a membrane composed of DOPC/DOEPC/perylene, 79:20:1 spreaded on DTS functionalized AAO.

Muscular F-Actin was added to the membrane $(\sim 0.7 \mu \mathrm{M})$ and incubated overnight at $4^{\circ} \mathrm{C}$ in F-buffer $\left(50 \mathrm{mM} \mathrm{KCl}, 0.2 \mathrm{mM} \mathrm{MgCl}_{2}, 0.1 \mathrm{mM}\right.$ EDTA, $0.1 \mathrm{mM} \mathrm{NaN}$, $20 \mathrm{mM}$ TRIS/HCl, pH 7.4). CLSM images (Fig. 7.12 A) show the heterogeneous green fluorescence of the Alexa Fluor488 phalloidin labeled F-actin atop the pores ( $x-y$ plane). The $x-$ $\mathrm{z}$ plane images show either a bright green fluorescence only atop the pores (Fig. 7.12 B, upper image) or a pore interior that was partly filled with F-actin (Fig. 7.12 B, lower image). Fluorescence images of neat perylene labeled membranes ( $\lambda_{\mathrm{ex}}=488 \mathrm{~nm}$ ) reveal that the dark green background fluorescence observed in the cross sections was not a result of F-actin fluorescence but of the perylene fluorescence. In fact, the regions of empty and partly filled pores were heterogeneously distributed over the samples in domains that could extend over several hundred micrometers. Using an atomic force microscopy (AFM) setup in combination with a fluorescence microscope would allow to select the desired region and investigate the mechanical properties of either F-actin only atop or also inside the pores.

Surprisingly, F-actin integration in or exclusion from the pore interior was neither a function of the pore diameter nor the DOEPC content in case of the applied range (30- 
$50 \mathrm{~mol} \%$ DOEPC). All parameters ( $d=35 \mathrm{~nm}$ and $d=45 \mathrm{~nm}$ and $30 \mathrm{~mol} \%$ and $50 \mathrm{~mol} \%$ DOEPC) lead to the same results that are represented in Fig. 7.12. However, a control experiment with pure DOPC membrane showed that no filaments were attached on the AAO substrate, neither atop the pores, nor in the pore interior.

\section{Optical Waveguide Spectroscopy Experiments}

Optical waveguide spectroscopy (OWS) experiments were performed and analyzed to quantify the F-actin adsorption within APTES and DOEPC functionalized AAO pores.
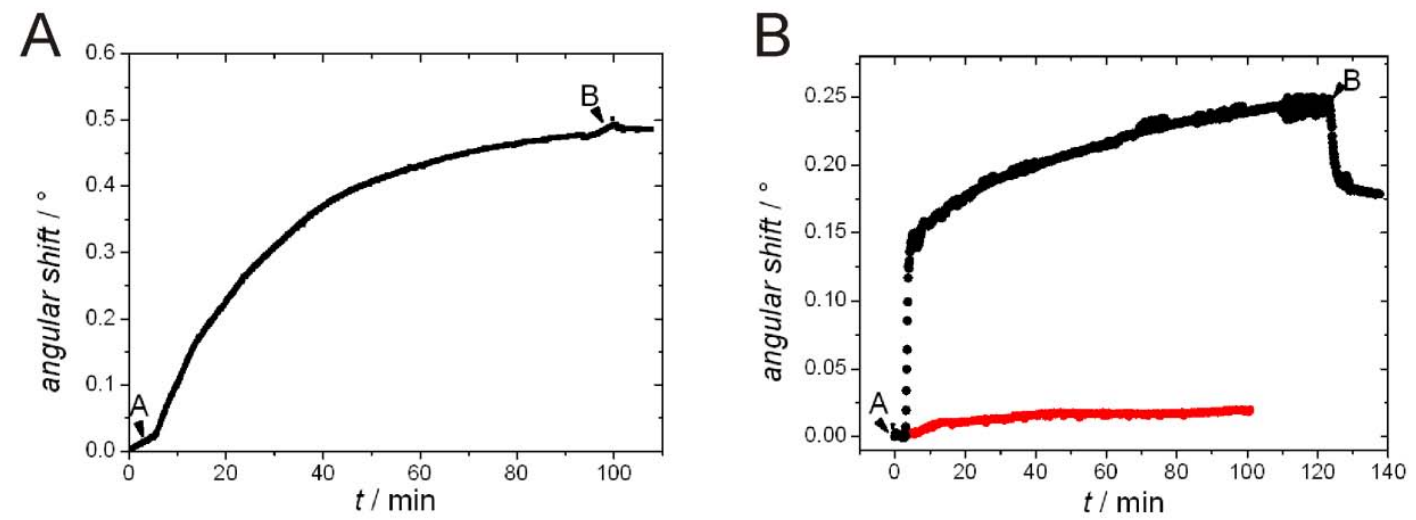

Fig. 7.13 (A) Time course of the angular shift after spreading SUVs composed of DOPC/DOEPC, 7:3 (A) on DTS functionalized AAO $(d=60 \mathrm{~nm}, h=3.5 \mu \mathrm{m})$ and F-buffer $(50 \mathrm{mM} \mathrm{KCl}, 0.2 \mathrm{mM} \mathrm{MgCl}, 0.1 \mathrm{mM}$ EDTA, $0.1 \mathrm{mM} \mathrm{NaN}_{3}, 20 \mathrm{~mm}$ TRIS/HCl, pH 7.4) rinsing (B). (B) Time course of the angular shift after the addition of $1 \mu \mathrm{M}$ F-actin stabilized with phalloidin (A) to AAO covered with APTES (black) or with membrane containing DOPC/DOEPC, 7:3 (red).

Spreading of SUVs composed of DOPC/DOEPC, 7:3 (Fig. $7.13 \mathrm{~A}$ ) resulted in an angular shift of $0.5^{\circ}$, which corresponds approximately to a thickness shift of $d=2 \mathrm{~nm}$ indicating the formation of a lipid monolayer atop the DTS functionalized AAO surface.

The F-actin adsorption inside AAO pores $(d=60 \mathrm{~nm}, h=3.5 \mu \mathrm{m})$ that were functionalized with either APTES or the DOEPC containing membrane is depicted in Fig. 7.13 B. The black curve shows the time course of the angular shift after the addition of $1 \mu \mathrm{M}$ 
F-actin to APTES functionalized AAO (angular shift $=0.18^{\circ}$ ), while the red curve corresponds to the time course of the angular shift after the addition of $1 \mu \mathrm{M}$ F-actin to the DOEPC containing membrane (angular shift $=0.03^{\circ}$ ). The apparent difference between the two adsorption curves confirms the results obtained by means of CLSM experiments. While F-actin is incorporated in the pores that are functionalized with APTES, it is almost entirely excluded from the pore interior, if a DOEPC containing membrane is lining the porous surface.

\subsubsection{F-actin Adsorption Within Porous AAO with an erzin/PIP ${ }_{2}$ Con- taining Membrane}

As a model that best mimics the nature of microvilli, a layer by layer assembly of a solid supported hybrid membrane containing the natural ezrin receptor lipid $\mathrm{PIP}_{2}$, ezrin and F-actin was aimed to build up (route three in Fig. 7.6.) First, $\mathrm{PIP}_{2}$ containing membrane formation as well as ezrin binding were characterized and controlled by means of CLSM and OWS as perquisite to controlled F-actin adsorption.

\section{Protein Expression and Purification}

Ezrin $\left(M=69.5 \mathrm{kDa}, 586\right.$ amino acids, Met $\left.^{1}-\mathrm{Leu}^{586}\right)$ was expressed in Escherichia coli (strain: BL21(DE3)pLysS) with a hexahistidine tag at the N-terminus. A hexahistidine tag is commonly associated with the C-terminus. In the case of ezrin, however, this region harbors a binding site for F-actin (Ile $\left.{ }^{553}-\mathrm{Leu}^{586}\right)$. Introducing an artificial tag at this region would most likely mask the binding site or at least modify its F-actin binding ability. Also, the natural binding site that links ezrin with the plasma membrane (FERM domain, Met $^{1}-\mathrm{Asp}^{298}$ ) is located at the $N$-terminus. Thus, it is beneficial to use a $N$-terminal hexahistidine tag for membrane immobilization studies. Nevertheless, a $N$ terminal tag implicates certain drawbacks regarding the protein purification. As proteins are synthesized from the $N$ - to the $C$-terminus, ezrin fragments, which are not 
completely synthesized also carry a hexahistidine tag and are thus eluted together with the full length protein.

Fig. 7.14 shows a SDS-PAGE gel of the molecular marker (lane M), the crude cell lysate (lane L) and an ezrin elution fraction (lane E). Several bands of lower molecular weight ( 50-32 kDa, lane E) indicate the presence of ezrin fragments with a hexahistidine tag in the elution fraction. However, these band are way less intensive than the main ezrin band at $M \approx 80 \mathrm{kDa}$. It can thus be assumed that their presence would not mainly affect the experimental performances planned with ezrin.

Interestingly, the molecular mass of ezrin determined from the SDS-gel differs from the one calculated on the basis of the amino acid sequence $(\Delta M \approx 10 \mathrm{KDa})$. This deviation is well-known in the literature and can be explained by the relatively high intrinsic charges of ezrin $(\mathrm{pI}=5.94)$ and the heptaproline sequence buried in its $\alpha$-helical region. ${ }^{31}$ The accumulation of proline is known to impose conformational changes that may influence the electrophoretic mobility of the protein. ${ }^{32}$ 


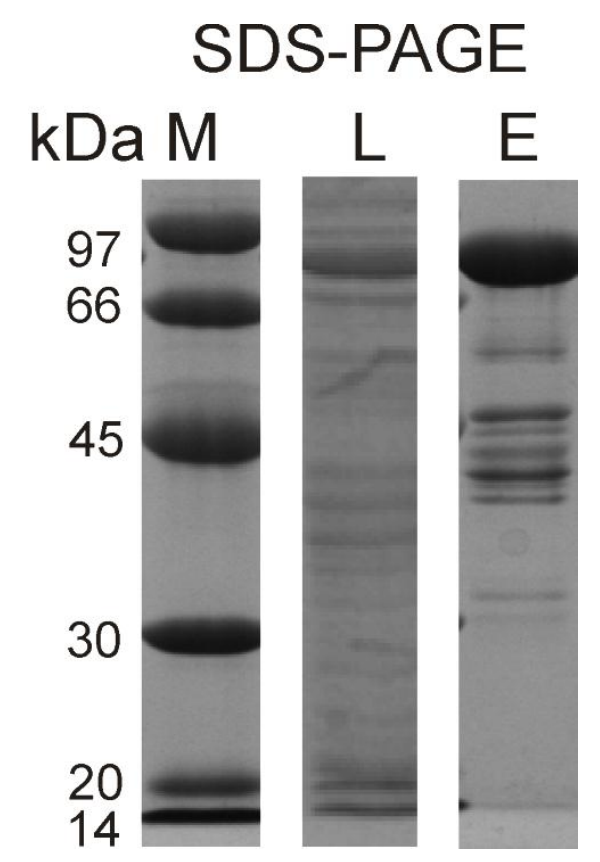

Fig. 7.14 SDS gels of ezrin protein fractions. The Coomassie stained SDS gel shows the molecular marker (M), cell lysate (L) and elution fraction (E) of ezrin. According to the SDS gel the molecular mass of ezrin is approx. $M=80 \mathrm{kDa}$.

\section{Optical Waveguide Sectroscopy Experiments}

OWS experiments were performed in order to verify proper spreading of $\mathrm{PIP}_{2}$ containing vesicles on the AAO surface that was functionalized with DTS followed by the subsequent binding of ezrin to the $\mathrm{PIP}_{2}$ receptor lipid.

In Fig. $7.15 \mathrm{~A}$, the time course of the angular shift after addition of SUVs composed of POPC/PIP2, 9:1 in E1-buffer (50 mM KCl, 20 mM TRIS/HCl, 0.1 mM EDTA, 0.1 mM NaN3, $\mathrm{pH}$ 7.4) is shown. After rinsing with buffer an angular shift of $0.58^{\circ}$ was detected. An average thickness of $d=(1.5 \pm 0.6) \mathrm{nm},(\mathrm{n}=4)$ was determined for the POPC/PIP $2,9: 1$ monolayer. This value that is slightly lower than expected for a lipid monolayer $(d=2 \mathrm{~nm})$, but in accordance with the one measured by means of surface plasmone resonance technique (chapter 4.3.2, page 119) for DOPC/DOGS-NTA-Ni, 9:1 membranes $(d=(1.2 \pm 0.5) \mathrm{nm}, \mathrm{n}=3)$. 

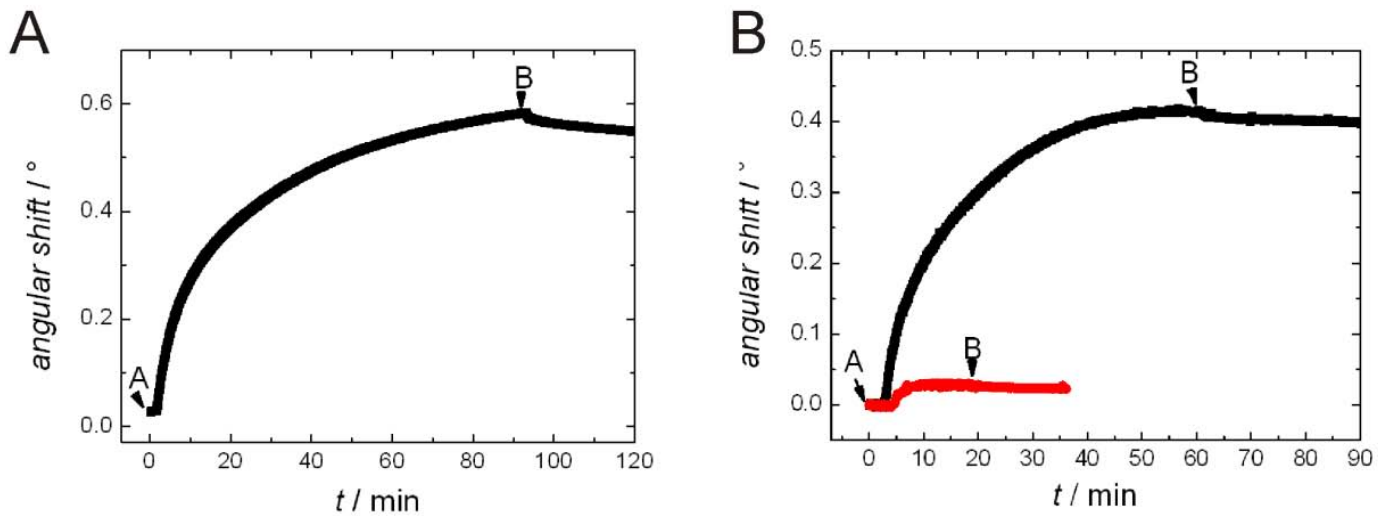

Fig. 7.15 (A) Time course of the angular shift after spreading of SUVs composed of POPC/PIP2, 9:1 (A) on AAO pores functionalized with DTS and E1 buffer $(50 \mathrm{mM} \mathrm{KCl}, 20 \mathrm{mM}$ TRIS/HCl, $0.1 \mathrm{mM}$ EDTA, $0.1 \mathrm{mM}$ $\mathrm{NaN}_{3}$, pH 7.4) rinsing (B). (B) Time course of the angular shift after the addition of $0.7 \mu \mathrm{M}$ ezrin (A) to a POPC/ PIP2, 9:1 membrane (black) and to a pure POPC membrane (red) that line the pore interior of porous $\mathrm{AAO}$ and rinsing with $\mathrm{E} 1$ buffer (B).

A representative time course of the angular shift after the addition of $0.7 \mu \mathrm{M}$ ezrin to a POPC/PIP 2, 9:1 membrane in E1 buffer is depicted in Fig. 7.15 B. Buffer rinsing only led to a slight decrease of the angular shift, which demonstrates an almost irreversible, tight ezrin-PIP 2 binding (black curve). The final angular shift after buffer rinsing was $0.4^{\circ}$, which corresponds to an ezrin thickness of $1.8 \mathrm{~nm}$. A control experiment, at which $0.7 \mu \mathrm{M}$ ezrin was given to a pure POPC membrane, only lead to a slight angular shift of $0.03^{\circ}$ (red curve).

\section{Ezrin Labeling}

To visualize the ezrin-PIP 2 binding on planar and porous surfaces by means of CLSM, ezrin was fluorescently labled using the dyes AlexaFluor488 C5-maleimide and TexasRed-maleimide (see chapter 3.3.6, page 52). Ezrin exhibits two cystein residues (Cys ${ }^{117}$ and $\mathrm{Cys}^{284}$ ), whose thiol groups are capable of reacting with the maleimide group of the respective fluorescent dye. Compared to other reagents used for thiol modification, maleimide groups are more selective to thiols and do not react with histidine or methi- 
onine residues. ${ }^{33}$ The labeled protein fractions were analyzed by means of SDS-PAGE and $\mathrm{UV} / \mathrm{Vis}$ spectroscopy.

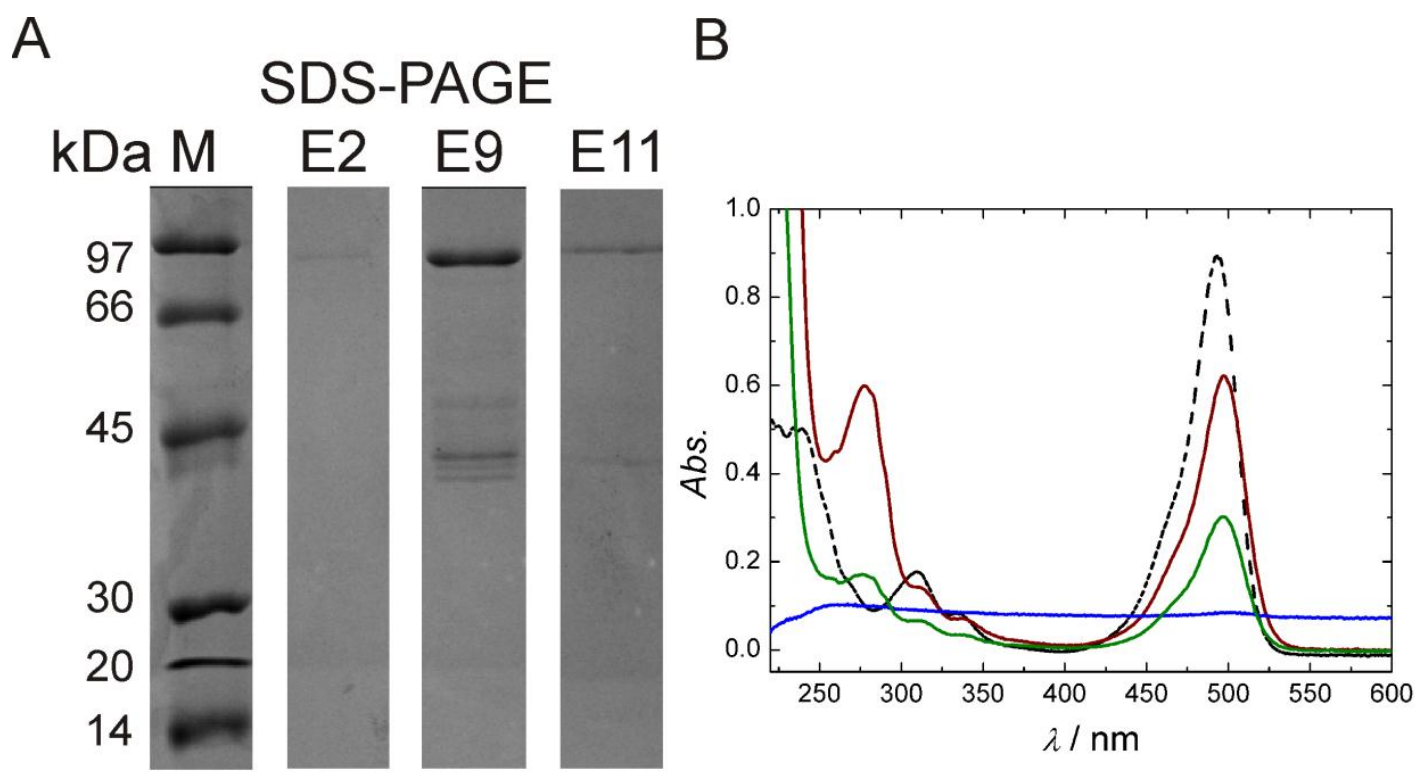

Fig. 7.16 (A) SDS-PAGE gel showing the molecular marker (M) and elution fractions E2, E9 and E11 after fluorescence labeling of ezrin. (B) UV/VIS spectra of different elution fractions of ezrin labeled with Alexa Fluor488 C5-maleimide: pure dye (black dashed line), fraction 2 (bue), fraction 8 (red) and fraction 11 (green).

Fig. 7.16 shows the SDS-PAGE gel (A) and UV/Vis spectra (B) of elution fractions of ezrin labeled with AlexaFluor488 C5-maleimide along with the pure dye spectrum (black dashed line, $\lambda_{\mathrm{ex}}=493 \mathrm{~nm}$ ). Obviously, neither dye nor protein can be verified by means of the SDS gel or the UV/Vis spectrum in fraction 2 (lane E2; blue line). However, fraction 9 shows a strong band at $M \approx 90 \mathrm{kDa}$ in the SDS-PAGE gel (lane E9) and maxima at $\lambda_{\mathrm{ex}}=280$ and $493 \mathrm{~nm}$ (red line), demonstrating the presence of AlexaFluor488 C5-maleimide labeled ezrin. The SDS gel further shows, that no ezrin fragmentation occurred during the reaction and that the molecular mass is slightly higher compared to the unlabeled ezrin, an effect that might be due to the negatively charged dye (see Fig. 3.11). While in fraction 9 both maxima show nearly the same absorbance, the 
absorbance of the dye at $\lambda_{\mathrm{ex}}=493 \mathrm{~nm}$ is significantly higher in fraction 11 compared to the protein absorbance at $\lambda_{\mathrm{ex}}=280 \mathrm{~nm}$.

Protein concentration (cezrin) and the degree of labeling (DOL) were calculated using eq. 7.1 and eq. 7.2:

$$
\begin{array}{r}
c_{\text {ezrin }}=\frac{A_{280}-\left(A_{493} \cdot C F\right)}{\varepsilon_{\text {ezrin }}}, \\
D O L=\frac{A_{493} \cdot \varepsilon_{\text {ezrin }}}{\left(A_{280}-A_{493} \cdot C F\right) \cdot \varepsilon_{\text {dye }}},
\end{array}
$$

with $\varepsilon_{\text {ezrin }}=66,900 \mathrm{M}^{-1} \cdot \mathrm{cm}^{-1}, \quad \varepsilon_{\text {dye }}=71,100 \mathrm{M}^{-1} \cdot \mathrm{cm}^{-1}$ and the correction factor $\mathrm{CF}=0.1=A_{280} / A_{493}$ (from the dye UV/VIS spectrum).

Applying these equations, it became obvious that fraction 9 contained ezrin with a high concentration $(c=8 \mu \mathrm{M})$ and a $D O L=1$. In fraction 11 on the contrary, the dye was enriched $(D O L=2)$ and the ezrin concentration was reduced to $c=2 \mu \mathrm{M}$. This suggests that the amount of unbound dye in fraction 11 and all further fractions, where the DOI was even higher, was strongly increased. As unbound dye might affect experimental results, these fractions were not used for fluorescence microscopy experiments.

Alexa Fluor488 C5-maleimide labeled ezrin was obtained with a mean concentration of $C_{\text {ezrin }}=6.8 \mu \mathrm{M}$ (fraction 4-10) and a mean degree of labeling of $D O L=1.0$.

\section{Ezrin Adsorption on Planar and Porous Membranes by Means of CLSM}

To further demonstrate the specificity of the ezrin $\mathrm{PIP}_{2}$ membrane interaction, fluorescently labeled ezrin was added to planar solid supported hybrid membranes with a DTS-silicon support as well as to membranes formed on AAO that was functionalized with DTS (experiments on planar membranes were performed by Julia Braunger). In 
case of the latter, ezrin adsorption inside the porous areas was recorded in a time resolved manner by means of the CLSM.

Fig. 7.17 A (upper images) shows the fluorescence of perylene (blue) and PIP2-TMR (red) of a membrane composed of POPC/PIP $/ \mathrm{PIP}_{2}-\mathrm{TMR} /$ perylene, 96:2.5:0.5:1. After the addition of $0.7 \mu \mathrm{M}$ AlexaFluor488 C5-maleimide labeled ezrin (2 h incubation) and rinsing with E1 buffer (50 mM KCl, 20 mM TRIS/HCl, 0.1 mM EDTA, 0.1 mM NaN3, pH 7.4) the green fluorescence of the labeled ezrin was visualized atop the membrane (Fig. 7.17 A, lower image). While the blue perylene fluorescence remained homogeneously dispersed after ezrin addition, the red PIP 2 -TMR fluorescence was heterogeneously distributed in the membrane and overlapped with the green ezrin fluorescence. The correspondence between the red $\mathrm{PIP}_{2}-\mathrm{TMR}$ and the green ezrin fluorescence proves that ezrin specifically binds to $\mathrm{PIP}_{2}$

A time course of TexasRed-maleimide labeled ezrin addition to POPC/PIP 2, 9:1 membranes formed on a AAO surface functionalized with DTS is shown in Fig. 7.17 B. All images are $z$-x-plane images showing the pore bottom (white dashed line) and the whole pore interior. The first image at $-5 \mathrm{~s}$ shows the weak red background fluorescence of the AAO covered with the $\mathrm{PIP}_{2}$ containing membrane before ezrin addition. After addition of $0.3 \mu \mathrm{M}$ labeled ezrin and continuous increase of fluorescence intensity inside the porous area was monitored. 

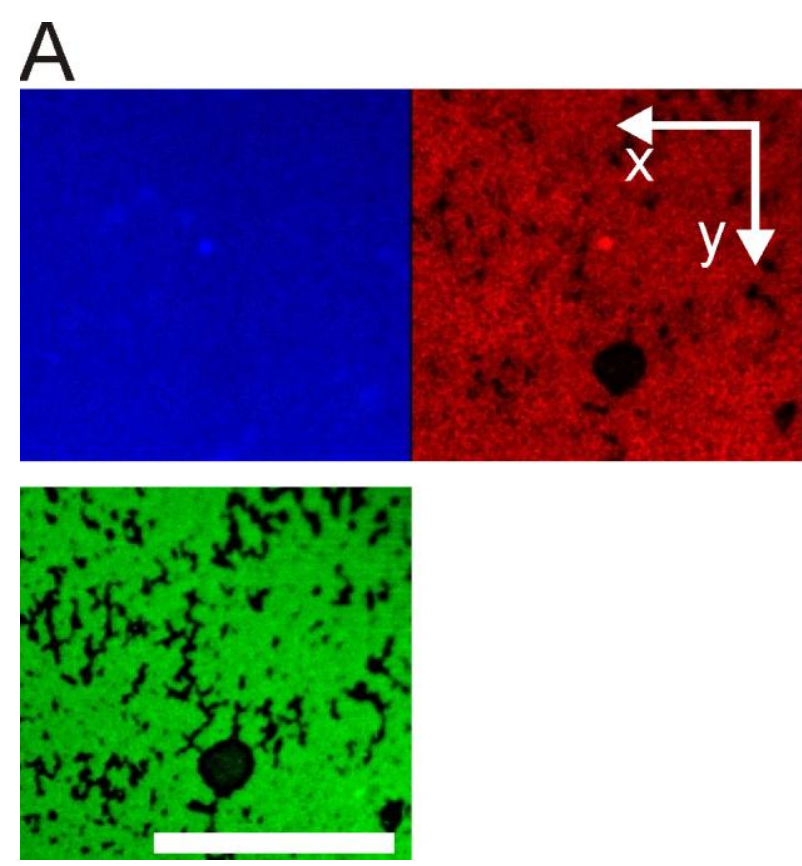
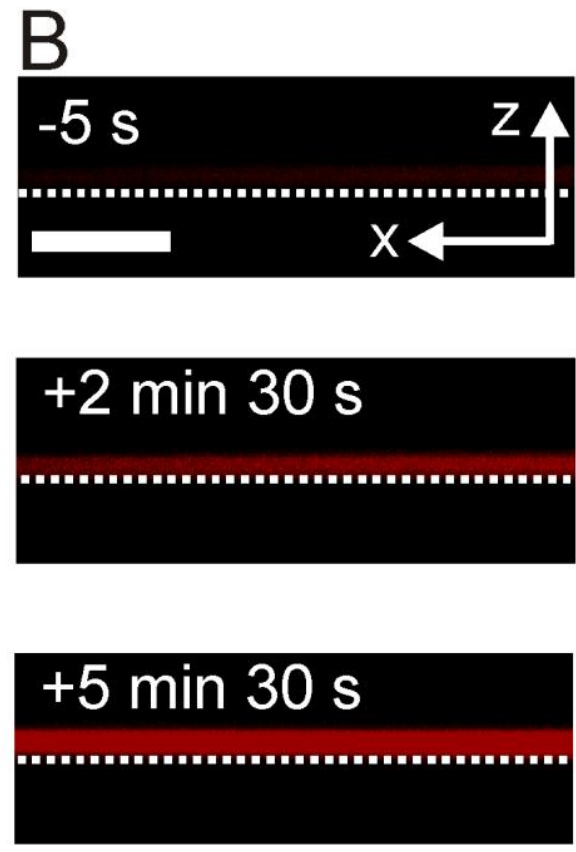

Fig. 7.17 (A) Fluorescence $x-y$ plane image of a solid supported hybrid membrane composed of

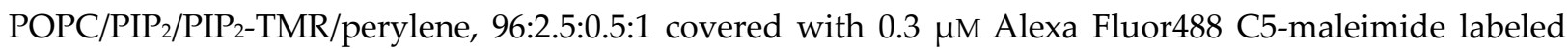
ezrin with the blue perylene fluorescence (upper left), the red $\mathrm{PIP}_{2}-\mathrm{TMR}$ fluorescence (upper right) and green ezrin fluorescence (lower left). The fluorescence images were kindly provided by Julia Braunger, who performed the CLSM experiments. (B) Fluorescence $y-z$ plane images showing the evolution of $0.3 \mu \mathrm{M}$ Texas Red maleimide labeled ezrin (red) adsorption on a POPC/ PIP2, 9:1 membrane spread on DTS functionalized AAO. Scale bars: $20 \mu \mathrm{m}$.

Rinsing with buffer after $1.5 \mathrm{~h}$ of ezrin incubation lead to no significant loss of fluorescence intensity. Both experiments demonstrate the specificity of the ezrin $\mathrm{PIP}_{2}$ binding and confirms the results obtained by means of OWS experiments (Fig. 7.15).

\section{F-actin Adsorption Within Ezrin/PIP2-membrane Covered AAO Pores}

In the next step, F-actin adsorption onto the ezrin covered membrane was monitored. Fig. 7.18 A shows the blue perylene fluorescence of a POPC/PIP2/perylene, 89:10:1 membrane on an AAO surface that was functionalized with DTS. Lateral mobility of this hybrid membrane was demonstrated by means of FRAP experiments (Fig. 7.18 B). The time course of the recovery was similar to those found for DOEPC/DOPC membranes (see 7.3.3) and to those from the literature. ${ }^{29}$ 

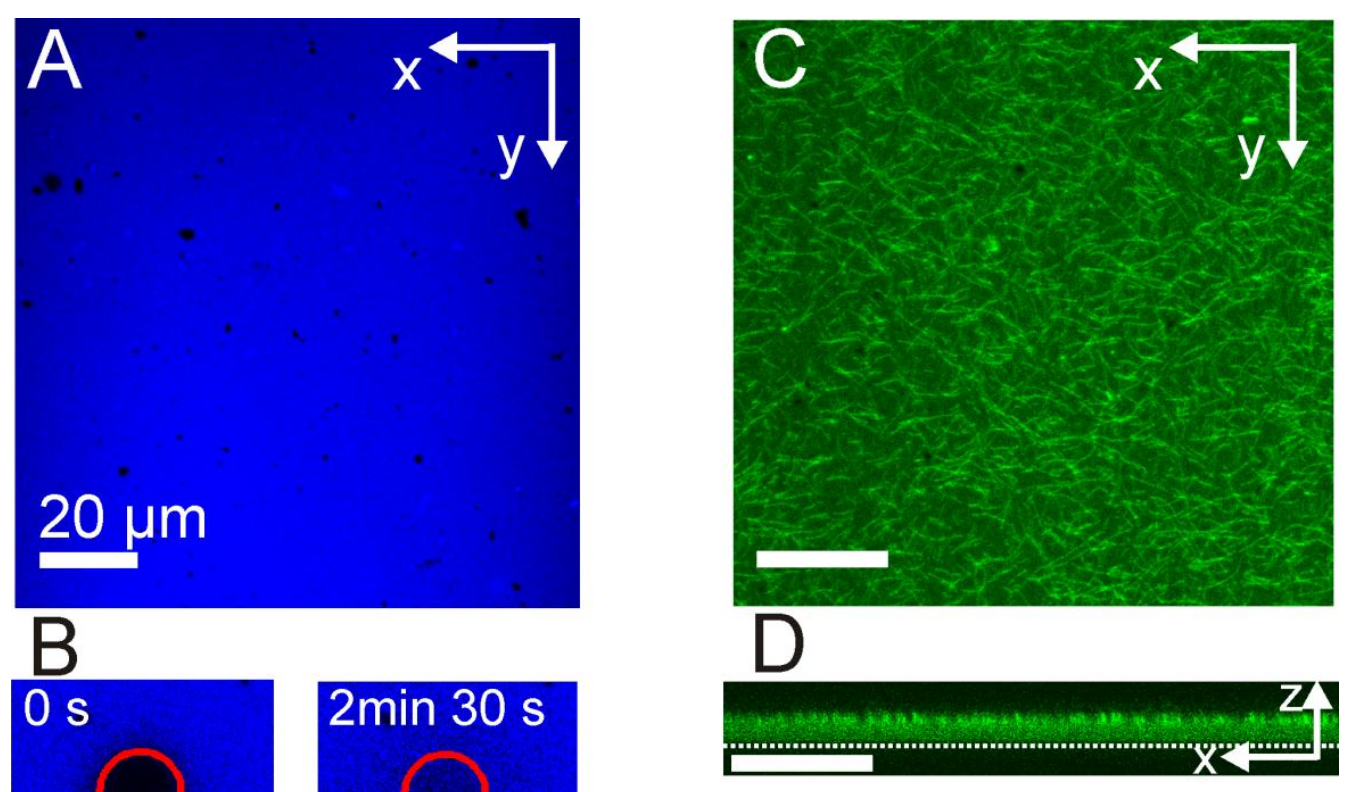

Fig. 7.18 (A) Fluorescence $x-y$ plane image of a solid supported hybrid membrane composed of POPC/PIP2/perylene, 89:10:1 on AAO functionalized with DTS. (B) Exemplary fluorescence images showing the time course of the recovery of fluorescence intensity after bleaching. (C) Fluorescence $x-y$ plane image and $\mathrm{x}-\mathrm{z}$ plane image (D) of F-actin labeled with AlexaFluor488 phalloidin on a $\mathrm{POPC} / \mathrm{PIP}_{2} /$ perylene, 89:10:1 membrane covered with ezrin (pore bottom is marked with a white, dashed line).

After incubation of $0.7 \mu \mathrm{M}$ ezrin $(1.5 \mathrm{~h}$ at room temperature using a flow through device), 0.5 units $(\sim 1 \mu \mathrm{M})$ of non muscular F-actin were added to the sample and incubated overnight at $4{ }^{\circ} \mathrm{C}$ in F-buffer $\left(50 \mathrm{mM} \mathrm{KCl}, 0.2 \mathrm{mM} \mathrm{MgCl}_{2}, 0.1 \mathrm{mM}\right.$ EDTA, $0.1 \mathrm{mM}$ $\mathrm{NaN}_{3}, 20 \mathrm{mM}$ TRIS/HCl, pH 7.4). Non muscular F-actin was used in this case, since cellular ezrin binds specifically to the $\beta$-isoform of non muscular F-actin with high affinity $\left(K_{\mathrm{D}}=46 \mathrm{nM}\right.$, stoichiometry 1:10, ezrin/actin). ${ }^{34}$ Although Roy et al. found that human recombinant ezrin binds to both $\alpha$ - and $\beta / \gamma$-actin with comparable affinity ${ }^{35}$, it was aimed in this work to mimic the cellular environment and conditions as best as possible, so that non muscular F-actin was chosen in this study. 
In Fig. $7.18 \mathrm{C}$ the green fluorescence of AlexaFluor488 phalloidin is depicted as an $\mathrm{x}-\mathrm{y}$ plane image together with an x-z plane image (Fig. $7.18 \mathrm{D})$ that shows the presence of F-actin in the AAO pore interior.

It was the idea to control the position of F-actin adsorption, i.e. to exclude the filaments from the pore interior by tuning the pore diameter $(d=35-65 \mathrm{~nm})$. However, it was not possible to exclude filamentous actin in a reproducible manner.

\subsection{Discussion}

In this work, a model system based on highly ordered nanoporous anodic aluminum oxide (AAO) was developed to mimic the microvilli of polarized epithelial cells (compare Fig. 7.5 and Fig. 7.6). Using a layer by layer assembly, three different routes were developed to build up systems, where the exclusion/inclusion of F-actin in the pore interior of the AAO could be governed (Fig. 7.6). In order to examine the contribution of F-actin bundles inside the pores on the mechanical properties of the overlying Factin network, the final aim was to investigate and compare the mechanical properties of both an F-actin network with and without underlying F-actin bundles by means of force spectroscopy measurements. Owing to the optical transparency of AAO substrates, the adsorption of F-actin and ezrin as well as vesicle spreading inside the AAO pores could be monitored in real time by means of optical waveguide spectroscopy (OWS) or confocal laser scanning microscopy (CLSM). Combining these two methods, it was possible to obtain quantitative information about the molecules adsorbed inside the pores (OWS) as well as to visualize the adsorbed material in the pore interior (x-z plane CLSM images) and atop the pores (x-y plane CLSM images).

First, F-actin adsorption atop/within AAO pores should be directed using a very simplified model system that allows specific F-actin binding. Due to its low pI ( 5.5) and relatively high negative charge density $(1 \mathrm{e} / 2.5 \AA$ ), F-actin can be electrostatically ad- 
sorbed to positively charged lipids and monolayers. ${ }^{36,37}$ Renault et al. demonstrated that actin polymerization can in fact be induced by a positively charged surface. ${ }^{37}$ Using ellipsometry and microscopic techniques, they were able to characterize the adsorption and polymerization of actin on Langmuir monolayers with a $\sim 30 \mathrm{~mol} \%$ content of positively charged amphiphiles. Other groups reported the adsorption of F-actin to positively charged lipid monolayers and liposomes. ${ }^{38,39}$ Here, we used positively charged aminopropyl-triethoxysilane (APTES) to bind actin filaments to the porous surface of AAO films. The specificity of binding was demonstrated by appropriate control experiments (data not shown). Using AAO substrates lacking APTES functionalization, no Alexa Fluor488 phalloidin labeled F-actin was observed neither inside nor atop the AAO pores. This finding demonstrates a specific F-actin-APTES interaction. Control of F-actin exclusion from and inclusion in the pore interior of the AAO substrates should only be gained by tuning the AAO pore diameter from $25 \mathrm{~nm}$ to $65 \mathrm{~nm}$. However, only the tendency of a reduced F-actin pore penetration with decreasing pore diameter was found. Exclusion of actin filaments from the AAO pores in a reproducible manner could not be achieved.

Regarding the actin filament dimensions, the mean diameter of unbundled F-actin is $d=7 \mathrm{~nm}$ (maximal diameter, $d_{\max }=9 \mathrm{~nm}$ ), while the mean length of in vitro polymerized actin is very heterogeneous, ranging from $0.5-10 \mu \mathrm{m}$ depending on the G-actin concentration, temperature and ionic strength. ${ }^{40}$ With a G-actin concentration of $c=0.5 \mathrm{mg} / \mathrm{ml}$ at $25^{\circ} \mathrm{C}$ similar to our conditions $(c=0.4 \mathrm{mg} / \mathrm{ml}$, room temperature) an average filament length of $l=1 \mu \mathrm{m}$ and a rather heterogeneous distribution (many filaments with $l<0.1 \mu \mathrm{m})$ was determined by Kawamura et al. ${ }^{40}$ Bundling F-actin can only be achieved using natural actin binding proteins (ABPs), artificial crosslinker (i.e. glutaraldehyde) or divalent cations $\left(\mathrm{Ca}^{2+}, \mathrm{Mg}^{2+}\right) \cdot{ }^{41,42} \mathrm{~K}$ won et al. reported that in vitro polymerized actin forms bundles with a mean thickness of $d \approx 30 \mathrm{~nm}$, if polylysine is used as crosslink agent. ${ }^{43}$ However, it can be strongly assumed that F-actin is incapable of forming bundles under the applied experimental conditions, as electrostatic repulsions disfavor their assembly. 
Different $x-y$ plane fluorescence images show the variable nature of F-actin adsorbed on AAO pores (Fig. 7.8) and further confirm the heterogeneity of the F-actin length distribution. However, a heterogenous length distribution alone would not explain the variable nature of actin filaments in different experiments, since all experiments were performed in an identical manner. Bergeron et al. investigated the ion-dependence of the polymerization kinetics of both non-muscular actin isoforms. ${ }^{6}$ They found that polymerization was basically finished within 25-100 min and that the presence of $\mathrm{Ca}^{2+}$ rather decelerated the polymerization, while $\mathrm{Mg}^{2+}$ did not. In terms of the $\mathrm{Ca}^{2+}$ influence, these results were confirmed by Cooper et al.. ${ }^{44}$ They also demonstrated that actin polymerization was significantly accelerated applying actin capping proteins. In this work, actin polymerization was stopped after $30 \mathrm{~min}$ by adding phalloidin, which binds to F-actin with high affinity and prevents further polymerization and depolymerization. Slight variations of the temperature, actin or salt concentration might affect the nature of the resulting filaments. These findings might explain why no reproducibility was obtained using AAO with different pore diameters.

It was unexpected that experiments performed with orthogonally functionalized AAO films (see Fig. 7.10), did not alter the penetration behavior of F-actin compared to the AAO pores that were completely covered with APTES. Due to the incorporation of oxalic acid during the AAO film growth, the surface of non functionalized AAO films is expected to be negatively charged. ${ }^{45}$ It was assumed that negatively charged actin filaments would be repelled, due to repulsive electrostatic interactions. This notion was corroborated by the observation that non functionalized AAO films do not bind Factin. It can be hypothesized that as long as actin filaments experience attractive forces from the pore rims, they eventually collapse inside the pores. The remaining F-actin network atop the AAO pores could prevent desorption of already penetrated filaments by rinsing with buffer.

As a next step (see route 2 and 3 of Fig. 7.6), solid supported membranes were introduced that were either doped with the positively charged lipid DOEPC or the natural erzin receptor lipid $\mathrm{PIP}_{2}$. Lazzara et al. demonstrated that SUVs composed of 
DOPC/DOGS-NTA-Ni, 9:1 and SUVs doped with various biotin contents spread on porous $\mathrm{AAO}$, which had been functionalized with dodecyl-trichlorosilane (DTS). ${ }^{29}$ By means of OWS experiments, they determined a monolayer thickness of $d=(2.3 \pm 0.2 \mathrm{~nm})$, which is in accordance with the mean value of $\sim 2 \mathrm{~nm}$ determined by surface plasmon resonance (SPR) and other techniques. ${ }^{46,47}$ In this work OWS was used to determine monolayer thicknesses of $d=(1.5 \pm 0.6) \mathrm{nm}$ in case of the POPC/PIP $2,9: 1$ lipid mixture and $d \approx 2 \mathrm{~nm}$ for the DOPC/DOEPC, 7:3 mixture. The monolayer thickness of the $\mathrm{PIP}_{2}$ containing membrane is slightly lower than expected. Nevertheless, the values correlate with those of DOPC/DOGS-NTA-Ni, 9:1 membranes $(d=(1.2 \pm 0.5) \mathrm{nm})$ and of POPC/PIP $2,96: 4$ membranes $(d=(1.2 \pm 0.5) \mathrm{nm})$ determined by means of SPR experiments in this and another work. ${ }^{48}$ The long recovery time of the fluorophores after photobleaching of fluorescently labeled DOEPC and $\mathrm{PIP}_{2}$ containing membranes can be explained by the fact, that the actual distance of the diffusing molecules is extensively increased ( 170 times, assuming an inter pore distance of $\lambda c$ $\mathrm{c}=100 \mathrm{~nm}$, a pore dimater of $d_{\mathrm{P}}=65 \mathrm{~nm}$, and a pore depth of $h=7 \mu \mathrm{m}$ ) due to the three dimensional structure of the AAO pores. ${ }^{30}$ This factor does not linearly correlate with the recovery time difference of porous compared to flat surfaces. This is most likely because fluorophore bleaching interfered with the recovery time in case of the porous substrates, due to time scale of the measurement. Thus, quantitative evaluation of the lateral diffusion in terms of diffusion constant was not possible.

Selective F-actin exclusion from the pore interior $\left(d_{\text {pore }}=35-65 \mathrm{~nm}\right)$ was achieved using solid supported DOPC membranes with a 30-50 mol\% DOEPC content as a platform to electrostatically immobilize F-actin. OWS and CLSM experiments showed that compared to AAO functionalized with APTES considerably less filaments penetrated the porous area. Thus, we conclude that introducing surface change with a tunable content and the lateral mobility of the membrane surface is a quite more important control parameter to position F-action than varying the pore diameter alone.

As a final approach, it was aimed to mimic the situation within cellular microvilli as precise as possibly (route 3, Fig. 7.6). The specificity of ezrin binding to a POPC mem- 
brane doped with $10 \mathrm{~mol} \% \mathrm{PIP}_{2}$ was demonstrated by means of CLSM experiments using fluorescently labeled ezrin and OWS experiments, in which a height of approximately $d=1.8 \mathrm{~nm}$ was determined. The height of a $N$-terminal ezrin domain $(\mathrm{N}$ ERMAD, residues 1- 297, pdb code: 1NI2, see Fig. 7.19 A) is $\sim 4.1 \mathrm{~nm}$, while full length moesin (residues 1- 577, pdb code: 1EF1, see Fig. $7.19 \mathrm{~B}$ ) has a height of $\sim 5.4 \mathrm{~nm}$ as deduced from its crystal stucture (dimensions were measured by RasMol 2.5.7, freeware available online). ${ }^{24,19}$
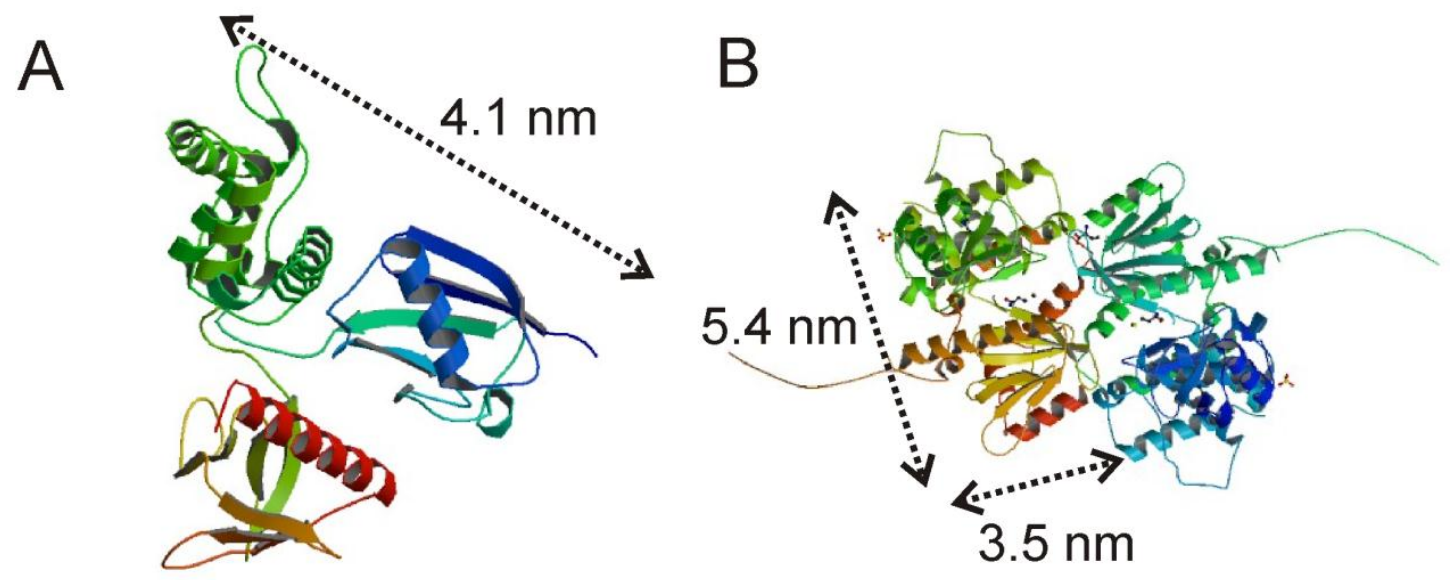

Fig. 7.19 (A) Crystal structure and dimension of N-terminal ezrin region N-ERMAD (pdb code: 1NI2) and (B) the dimer of full length moesin (pdb code: 1EF1) .

Comparing the ezrin dimensions derived from the crystal structures with the protein layer thickness of $d=1.8 \mathrm{~nm}$ determined from OWS experiments, a 33-45\% membrane coverage with ezrin can be calculated, which is close to the jamming limit of $54 \%$. Fluorescence images with Alexa Fluor488 C5-maleimide labeled ezrin on planar solid supported membranes composed of POPC/PIP $/ \mathrm{PIP}_{2}$-TMR/perylene, 96:2.5:0.5:1 showed that ezrin bound only to membrane areas that contain $\mathrm{PIP}_{2}$, shown by the overlapping fluorescence signals. This further demonstrated the specificity of the ezrin-PIP 2 interaction. It was expected that, since the experiments performed with DOEPC containing 
membranes showed an exclusion of F-actin, ezrin covered $\mathrm{PIP}_{2}$ containing membranes would behave likewise. Surprisingly, in this case, it was impossible to gain control over the F-actin exclusion from the pore interior from AAO pores functionalized with the PIP2-membrane-ezrin assembly.

Additionally attempts to image the AAO surface in air with an atomic force microscope (Fig. 7.20) showed that, even though a cantilever with a long and sharp tip was used, the penetration depth of the cantilever inside the pores was only about 10-15 nm (Fig. 7.20 C). This depth is the minimum limit, if one considers performing indentation experiments. Steltenkamp et al. imaged AAO pores with similar pore diameters. The penetration depth measured by this group was in good agreement with the one determined in our work. ${ }^{49}$ In buffer however, imaging was even more hindered, as the cantilever tip got easily damaged from the hard and rough AAO surface. These additional difficulties further encouraged us to switch the system to F-actin covered PSMs that span a larger pore area. First results that could be gathered using this system are presented in chapter 8 .
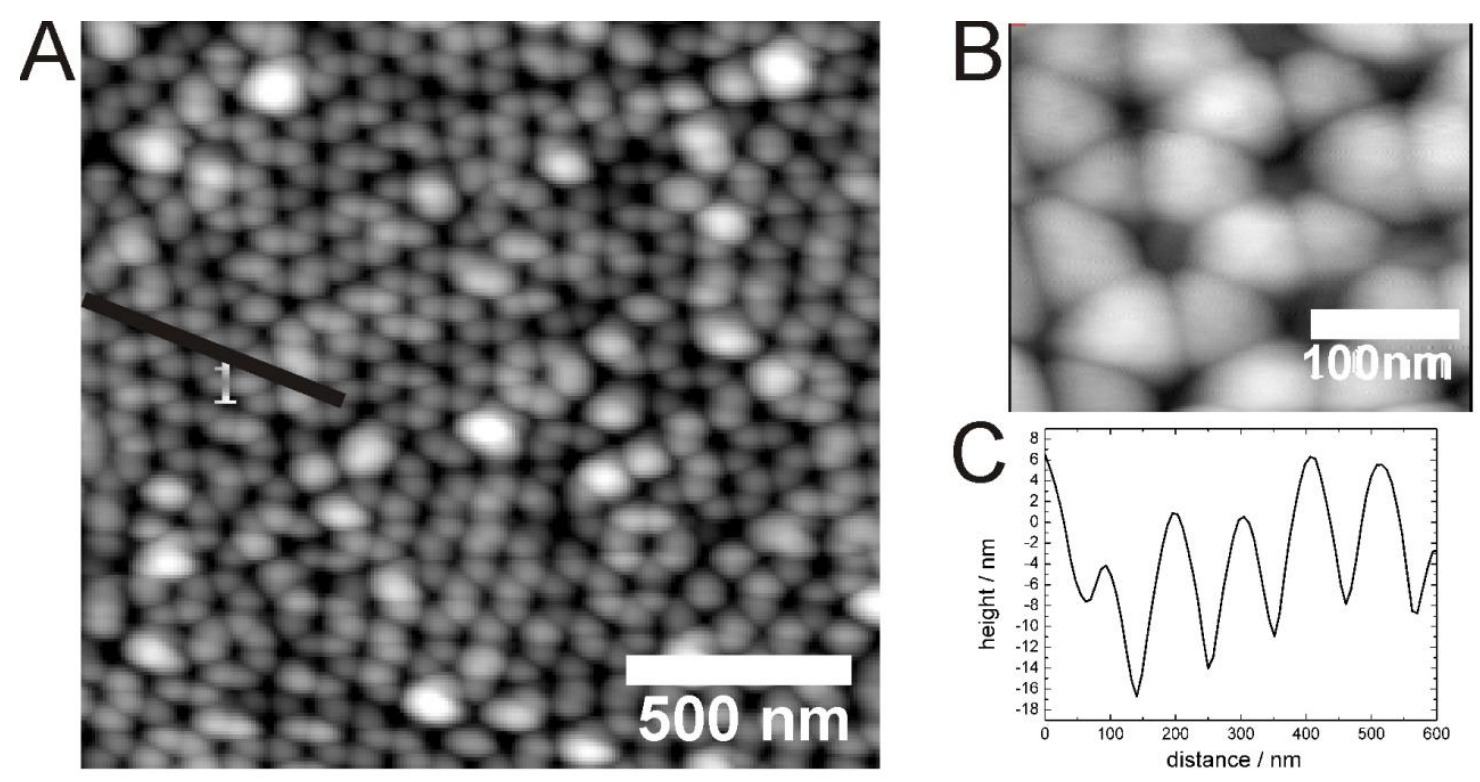

Fig. 7.20 (A and B) AFM image showing the topography of an AAO substrate with $d=45 \mathrm{~nm}$. (C) Height line profile corresponding to the black line in (A). 
Regarding that no reproducible F-actin exclusion from the pore interior could be achieved and that imaging of the AAO surface was unexpectedly challenging, the model system was changed in order to be able to perform force microscopy experiments. Instead of using a F-actin network polymerized above filamentous bundles inside the AAO pore interior, it was planned to investigate the impact of an F-actin network atop a pore spanning membrane (PSM) on porous silicon substrates (see chapter $8)$.

\subsection{Co-workers}

Mey, Ingo; Kramer, Corinna; Braunger, Julia; Austermann, Judith; Gerke, Volker 


\subsection{References}

[1] Wickstead, B., and Gull, K. (2011) Evolution: The evolution of the cytoskeleton. J. Cell. Biol. 194, 513-525.

[2] Fletcher, D. A., and Mullins, R. D. (2010) Cell mechanics and the cytoskeleton. Nature 463, 485-492.

[3] Podolski, J. L., and Steck, T. L. (1990) Length distribution of F-actin in Dictyostelium discoideum. J. Biol. Chem. 265, 1312-1318.

[4] Schmidt, A., and Hall, M. N. (1998) Signaling to the actin cytoskeleton. Annu. Rev. Cell Dev. Biol 14, 305-338.

[5] Herman, I. M. (1993) Actin isoforms. Curr. Opin. Cell Biol. 5, 48-55.

[6] Bergeron, S. E., Zhu, M., Thiem, S. M., Friderici, K. H., and Rubenstein, P. A. (2010) Ion-dependent Polymerization Differences between Mammalian-and Non muscle Actin Isoforms. J. Biol. Chem. 285, 16087-16095.

[7] Holmes, K. C., and Kabsch, W. (1991) Muscle proteins: actin. Curr. Opin. Struct. Biol. 1, 270-280.

[8] Holmes, K. C., Popp, D., Gebhard, W., and Kabsch, W. (1990) Atomic model of the actin filament. Nature 347, 44-49.

[9] P antaloni, D., Hill, T. L., Carlier, M. F., and Korn, E. D. (1985) A model for actin polymerization and the kinetic effects of ATP hydrolysis. Proc. Natl. Acad. Sci. U.S.A 82, 7207-7211.

[10] T D Pollard, a. J. A. C., Pollard, T. D., and Cooper, J. A. (1986) Actin and ActinBinding Proteins. A Critical Evaluation of Mechanisms and Functions. Annu. Rev. Biochem 55, 987-1035. 
[11] Köster, S. (2006) Biological Matter in Microfluidic Environment - from Single Molecules to Self-Assembly. Dissertation, Göttingen.

[12] W Kabsch, a. J. V., Kabsch, W., and Vandekerckhove, J. (1992) Structure and Function of Actin. Annu. Rev. Biophys. Biomol. Struct 21, 49-76.

[13] Staiger, C. J., and Blanchoin, L. (2006) Actin dynamics: old friends with new stories. Curr. Opin. Plant Biol. 9, 554-562.

[14] Carlier, M.-F. (1998) Control of actin dynamics. Curr. Opin. Cell Biol. 10, 45-51.

[15] Fehon, R. G., McClatchey, A. I., and Bretscher, A. (2010) Organizing the cell cor tex: the role of ERM proteins. Nat Rev Mol Cell Biol 11, 276-287.

[16] Tsukita, S., and Yonemura, S. (1999) Cortical actin organization: lessons from ERM (ezrin/radixin/moesin) proteins. J. Biol. Chem. 274, 34507-34510.

[17] Tsukita, S., Yonemura, S., and Tsukita, S. (1997) ERM proteins: head-to-tail regulation of actin-plasma membrane interaction. Trends Biochem. Sci. 22, 53-58.

[18] Neisch, A. L., and Fehon, R. G. (2011) Ezrin, Radixin and Moesin: key regulators of membrane-cortex interactions and signaling. Curr. Opin. Cell Biol. 23, 377-382.

[19] Pearson, M. A., Reczek, D., Bretscher, A., and Karplus, P. (2000) Structure of the ERM Protein Moesin Reveals the FERM Domain Fold Masked by an Extended Actin Binding Tail Domain. Cell 101, 259-270.

[20] McLaughlin, S., Wang, J., Gambhir, A., and Murray, D. (2002) PIP(2) and proteins: interactions, organization, and information flow. Annu Rev Biophys Biomol Struct 31, 151-175.

[21] Bosk, S., Braunger, J. A., Gerke, V., and Steinem, C. (2011) Activation of F-actin binding capacity of ezrin: synergism of $\mathrm{PIP}_{2}$ interaction and phosphorylation. Bio phys. J. 100, 1708-1717.

[22] Bretscher, A. (1999) Regulation of cortical structure by the ezrin-radixin-moesin protein family. Curr. Opin. Cell Biol. 11, 109-116. 
[23] Niggli, V., Andréoli, C., Roy, C., and Mangeat, P. (1995) Identification of a phosphatidylinositol-4,5-bisphosphate-binding domain in the N-terminal region of ezrin. FEBS Lett. 376, 172-176.

[24] Smith, W. J. (2002) Structure of the Active N-terminal Domain of Ezrin. Conformational and mobility changes identify keystone interactions. J. Biol. Chem. 278, 4949-4956.

[25] Turunen, O., Wahlström, T., and Vaheri, A. (1994) Ezrin has a COOH-terminal actin-binding site that is conserved in the ezrin protein family. J. Cell. Biol. 126, 1445-1453.

[26] Berryman, M., Franck, Z., and Bretscher, A. (1993) Ezrin is concentrated in the apical microvilli of a wide variety of epithelial cells whereas moesin is found primarily in endothelial cells. J. Cell. Sci. 105 ( Pt 4), 1025-1043.

[27] Anderson, J. H., and Taylor, A. B. (1973) Scanning and transmission electron microscopic studies of jejunal microvilli of the rat, hamster and dog. J. Morphol. 141, 281-291.

[28] Lazzara, T. D., Mey, I., Steinem, C., and Janshoff, A. (2011) Benefits and Limitations of Porous Substrates as Biosensors for Protein Adsorption. Anal. Chem 83, 5624-5630.

[29] Lazzara, T. D., Behn, D., Kliesch, T.-T., Janshoff, A., and Steinem, C. (2012) Phospholipids as an alternative to direct covalent coupling: surface functionalization of nanoporous alumina for protein recognition and purification. J. Colloid. Interf. Sci. 366, 57-63.

[30] Lazzara, T. D., Kliesch, T.-T., Janshoff, A., and Steinem, C. (2011) Orthogonal functionalization of nanoporous substrates: control of 3D surface functionality. ACS Appl Mater Interfaces 3, 1068-1076. 
[31] Gould, K. L., Bretscher, A., Esch, F. S., and Hunter, T. (1989) cDNA cloning and sequencing of the protein-tyrosine kinase substrate, ezrin, reveals homology to band 4.1. EMBO J. 8, 4133-4142.

[32] Turunen, O., Winqvist, R., Pakkanen, R., Grzeschik, K. H., Wahlström, T., and Vaheri, A. (1989) Cytovillin, a microvillar Mr 75,000 protein. cDNA sequence, prokaryotic expression, and chromosomal localization. J. Biol. Chem. 264, 1672716732.

[33] Invitrogen http://products.invitrogen.com/ivgn/product/A10254 (2012), cited on 2012 Dec. 9

[34] Yao, X., Cheng, L., and Forte, J. G. (1996) Biochemical characterization of ezrinactin interaction. J. Biol. Chem. 271, 7224-7229.

[35] Roy, C., Martin, M., and Mangeat, P. (1997) A dual involvement of the aminoterminal domain of ezrin in F- and G-actin binding. J. Biol. Chem. 272, 2008820095.

[36] Barfoot, R. J., Sheikh, K. H., Johnson, B. R. G., Colyer, J., Miles, R. E., Jeuken, L. J. C., Bushby, R. J., and Evans, S. D. (2008) Minimal F-Actin Cytoskeletal System for Planar Supported Phospholipid Bilayers. Langmuir 24, 6827-6836.

[37] Renault, A., Lenne, P.-F., Zakri, C., Aradian, A., Vénien-Bryan, C., and Amblard, F., (1999) Surface-Induced Polymerization of Actin. Biophys. J. 76, 1580-1590.

[38] Rioux, L., and Gicquaud, C. (1985) Actin paracrystalline sheets formed at the surface of positively charged liposomes. J. Ultra. Mol. Struct. R. 93, 42-49.

[39] Ward, R. J., Menetret, J.-F., Pattus, F., and Leonard, K. (1990) Method for forming two-dimensional paracrystals of biological filaments on lipid monolayers. J. Elec. Micr. Tech. 14, 335-341.

[40] Kawamura, M., and Maruyama, K. (1970) Electron microscopic particle length of F-actin polymerized in vitro. J. Biochem. 67, 437-457. 
[41] Haviv, L., Gov, N., Ideses, Y., and Bernheim-Groswasser, A. (2008) Thickness dis tribution of actin bundles in vitro. Eur. Biophys. J. 37, 447-454.

[42] Janmey, P. A. (1996) The Polyelectrolyte Nature of F-actin and the Mechanism of Actin Bundle Formation. J. Biol. Chem. 271, 8556-8563.

[43] Kwon, H. J., Tanaka, Y., Kakugo, A., Shikinaka, K., Furukawa, H., Osada, Y., and Gong, J. P. (2006) Anisotropic nucleation growth of actin bundle: a model for de termining the well-defined thickness of bundles. Biochemistry 45, 10313-10318.

[44] Cooper, J. A., and Pollard, T. D. (1985) Effect of capping protein on the kinetics of actin polymerization. Biochemistry 24, 793-799.

[45] Li, F., Zhang, L., and Metzger, R. M. (1998) On the Growth of Highly Ordered Pores in Anodized Aluminum Oxide. Chem. Mater 10, 2470-2480.

[46] Naumann, R., Jonczyk, A., Kopp, R., van Esch, J., Ringsdorf, H., Knoll, W., and Gräber, P. (1995) Incorporation of Membrane Proteins in Solid-Supported Lipid Layers. Angew. Chem. Int. Ed. Engl 34, 2056-2058.

[47] Rossi, C., Homand, J., Bauche, C., Hamdi, H., Ladant, D., and Chopineau, J. (2003) Differential mechanisms for calcium-dependent protein/membrane association as evidenced from SPR-binding studies on supported biomimetic membranes. Biochemistry 42, 15273-15283.

[48] Schwamborn, M. (2012) Charakterisierung der Bindung von F-Aktin an Ezrin mittels Oberflächenplasmonenresonanz. Masterthesis, Göttingen.

[49] Steltenkamp, S., Müller, M. M., Deserno, M., Hennesthal, C., Steinem, C., and Janshoff, A. (2006) Mechanical properties of pore-spanning lipid bilayers probed by atomic force microscopy. Biophys. J. 91, 217-226. 


\section{Mechanical Characterization of Filamen- tous Actin on Pore Spanning Membranes by Means of Force Spectroscopy}

\subsection{Abstract}

The cortical cytoskeleton is an essential mechanical component composed of filamentous actin (F-actin) and other regulatory factors, which control dynamical cellular processes including cellular division, motility and shape. The mechanical properties of Factin networks have been studied in vitro applying F-actin gel solutions or in vivo using living cells by means of various techniques.

Here, we present an extended in vitro assay based on artificial cell membrane mimics to investigate the mechanical properties of pore spanning membranes (PSMs) with an overlying F-actin network by means of atomic force microscopy (AFM). First, we used positively charged solid supported membranes (SSMs) to study the specificity of Factin binding by means of the surface plasmon resonance (SPR) technique and confocal laser scanning microscopy (CLSM). PSMs were prepared by spreading giant unilamellar vesicles (GUVs) composed of DOPC/DOPEC, 8:2 on porous silicon nitride substrates. F-actin binding was achieved via electrostatic interaction with the positively charged DOEPC lipid. By means of force mapping experiments of pure PSMs and PSMs with an overlying F-actin network the apparent spring constant $\left(k_{\mathrm{app}}\right)$ of both system could be determined to $k_{\mathrm{app}}=(1.0 \pm 0.2) \mathrm{mN} / \mathrm{m}$ for pure membranes and $k_{\mathrm{app}}=(1.4 \pm 0.5) \mathrm{mN} / \mathrm{m}$ for membranes with bound F-actin. While pure PSMs showed 
perfect elasticity, the appearance of a hysteresis between indentation and retraction curve observed in case of the membranes with bound F-actin, indicated energy dissipation due to viscoelestic properties of the membrane.

\subsection{Introduction}

Eukaryotic cells are supramolecular assemblies composed of different complex structures called organelles that serve as compartments with distinct metabolic functions and are embedded into membranes. The cytoskeleton is diversely organized and carries out three main functions: it spatially organizes cellular organelles, provides interand intracellular stability and enables dynamical cellular processes such as division, migration and differentiation. ${ }^{1,2}$ As the mechanical backbone of the cell, the cortical cytoskeleton determines structure and organization of the cell and is therefore eminent for regulating cellular functions. Although numerous proteins are involved in cytoskeletal organization, it is mainly composed of three polymers: microtubules, intermediate filaments and actin filaments (Fig. 8.1). 

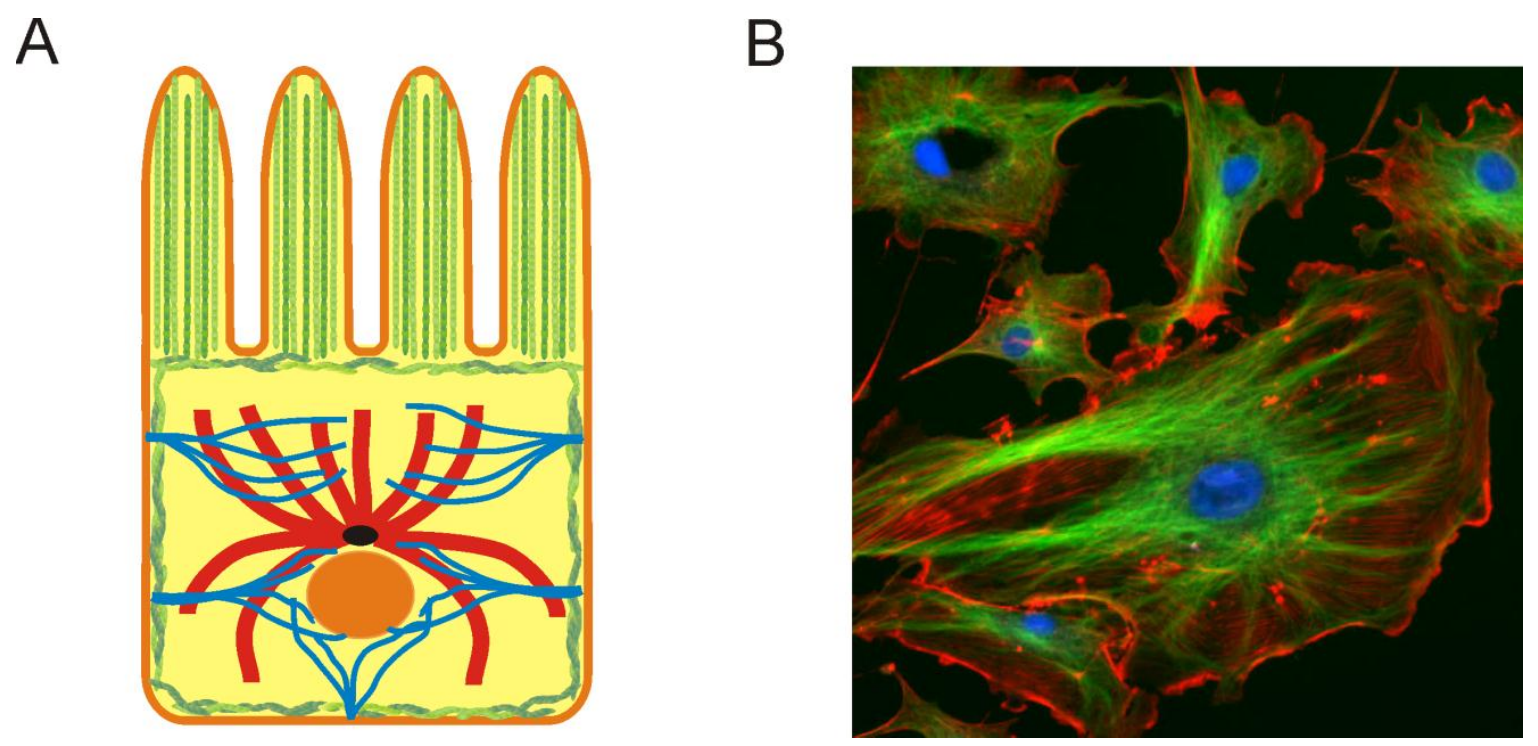

Fig. 8.1 (A) Schematic drawing showing the three components of the cytoskeleton within the cell: microtubules (red), intermediate filaments (blue) and actin filaments (green). (B) Fluorescence microscopy image showing the fluorescently labeled nucleus (blue), microtubules (green) and actin filaments (red) of endothelial cells. ${ }^{3}$

Microtubules are the stiffest of the three polymers and exhibit a persistence length of $l_{\mathrm{p}}=5 \mathrm{~mm}$. The persistence length is defined as the length scale, at which two segments of the polymer become uncorrelated, while the contour length of a polymer $\left(l_{c}\right)$ represents the length of a completely extended filament. They build up hollow cylinders (length, $l=25 \mu \mathrm{m}$, diameter, $d=25 \mathrm{~nm}$ ) as a result of polymerization of $\alpha$ - and $\beta$ tubulin heterodimers. Microtubules regulate intracellular traffic and genome segregation during mitosis. As the most flexible cytoskeletal polymer, the tertrameric intermediate filaments $(\mathrm{d}=10 \mathrm{~nm})$ are not directly involved in cellular dynamics, but play a structural role, providing the stability and mechanical strength of the cell. ${ }^{1,4}$

Actin filaments are semi flexible polymers, with a persistence length in the range of their contour length, $l_{\mathrm{p}}=13 \mu \mathrm{m}^{5}$, a mean diameter of $7 \mathrm{~nm}$ and a variable length ranging from $0.1-20 \mu \mathrm{m} .{ }^{6}$ The role of F-actin is highly associated with cellular dynamics and motility such as endocytosis and cytokinesis. These processes are mainly governed by the kinetics of filament turnover and the three dimensional assemblies of actin filaments. ${ }^{7}$ In order to control these dynamic processes and the actin network architecture, 
several actin binding proteins (ABPs) are involved (see Fig. 7.3 in chapter 7 on page 199).

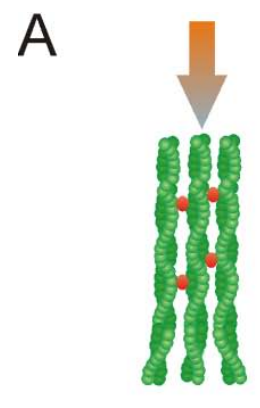

bundles

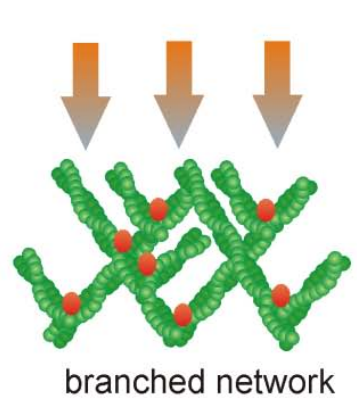

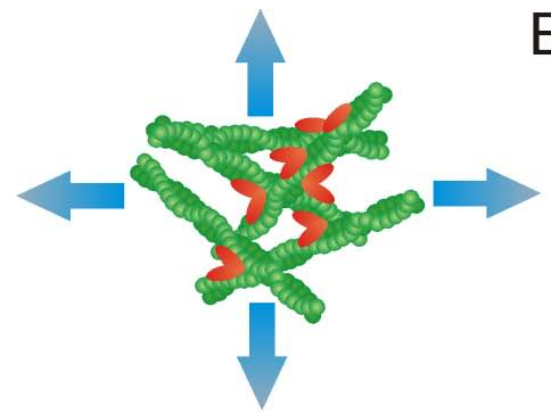

cortical network

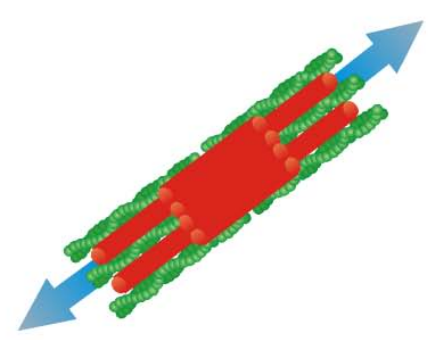

stress fibers
B
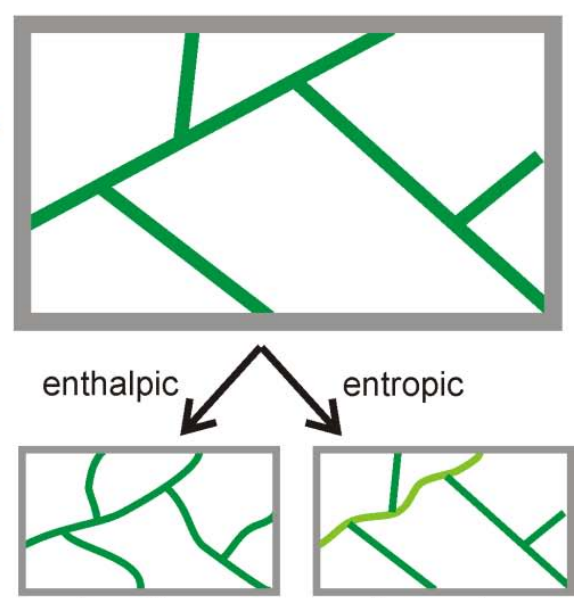

bending

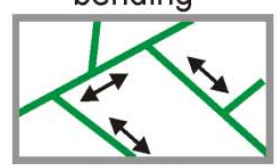

stretching

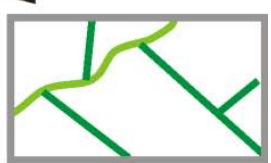

thermal

fluctuation

Fig. 8.2 (A) Schematic drawing of four types of F-actin architecture associated with different stress forces commonly encounted by these structures (compression: orange arrows, tension: blue arrows). ${ }^{1}$ (B) Schematic of entropic and enthalpic deformations of F-actin networks. ${ }^{8}$

Due to the steady dynamical filament turnover and network arrangement, actin filaments are constantly under the action of mechanical forces, arousing either from their interactions with cellular membranes or molecular motor proteins. The elastic contributions of actin filaments can be divided in entropic and enthalpic ones. Entropic elasticity mainly results from thermal fluctuations of individual filaments. Filaments i.e. resist extension, because this would reduce possible network configurations. Enthalpic elasticity on the contrary is caused by bending or stretching of the filament and the resulting changes in monomer spacing. Thus, it is obvious that the F-actin elasticity is mainly determined by the way actin filaments assemble into higher organized architectures. Three dimensional actin networks, for instance, stiffen when shear stress is applied, 
due a higher filament entanglement (entropic elasticity). ${ }^{1}$ However, enthalpic buckling of filaments after bending or stretching of branched networks leads to stress softening. ${ }^{8}$ Crosslinkers and other ABPs further influence the mechanical properties of actin bundles within networks. ${ }^{9}$ Depending on the cross linker protein and its molar ratio, different regimes of structural phases with distinct mechanical properties such as entangled actin solutions, isotropically cross linked filaments of bundled networks can be discriminated. ${ }^{10}$

The mechanical properties of in vitro polymerized actin gels have been studied extensively over the past decades by means of microrheology, dynamic light scattering or fluorescence techniques. ${ }^{11,12}$ Elasticity moduli $(E)$, which are in the range of $E \approx 1-10 \mathrm{kPa}$, were extracted as a function of network cross linking, density, filament length and other factors. ${ }^{8,13,12}$ To investigate the mechanical and viscoelastic properties of the F-actin cytoskeleton in vivo, experiments on a single cell level are often conducted using several techniques such as optical tweezers, micropipette aspiration and atomic force microscopy. ${ }^{14-16}$

By means of the AFM, the mechanical response of cells with an intact cytoskeleton as well as cells with a disrupted, non cross linked or non functional cytoskeleton can be investigated, giving information about the cytoskeletal influence on cellular mechanics. Rotsch et al. for instance, studied the changes of cytoskeletal structure and cellular mechanics on fibroblasts treated with cytochalasin B (Cyt B), a drug, that inhibits actin polymerization. By means of indentation maps, they demonstrated, that the cell's stiffness was remarkably reduced after $\mathrm{CytB}$ injection. ${ }^{17}$ Although many approaches have been developed to examine the mechanical and viscoelastic properties of F-actin in vitro and in vivo, these studies were confined to cellular systems or F-actin networks in solutions. Both systems, however bear certain disadvantages. Regarding cellular experiments the influence of single ABPs can be hardly discriminated, as the mechanical response of the cortical actin network is influenced by the interplay of a large number of different proteins inside a cell. In contrast, in vitro systems lack a membrane support, so that only F-actin mechanics can be measured. 
In the past, the AFM technique has been established as a powerful tool to image biological structures i.e. proteins, membranes and to quantitatively investigat biomolecular interactions. ${ }^{18-20}$. The mechanical properties of biological materials, adhesive cells, artificial pore spanning membranes and fixated basolateral cellular patches have been studied in terms of their mechanical response and viscoelastic behavior. ${ }^{21-25}$ Pore spanning membranes (PSMs) have been mechanically characterized as a function of the pore diameter, lipid composition and surface functionalisation..$^{26,27}$ To determine the bending modulus, tension and viscoelastic properties, force indentation experiments with high lateral resolution were conducted.

Here, we present an extended approach to study the mechanical properties of pore spanning membranes coupled to a F-actin network (Fig. 8.3). Theses cellular membrane mimics were investigated in terms of their elastic behavior by applying force indentation maps using an atomic force microscope. Pore spanning membranes were prepared on hydrophilically functionalized gold coated silicon nitride pores (pore diameter, $d=1.2 \mu \mathrm{m}$ ) by spreading giant unilamellar vesicles (GUVs). F-actin was electrostatically immobilized on the positively charged membrane (positive charge was provided using a lipid mixture of DOPC doped with 20 mol\% DOEPC). 


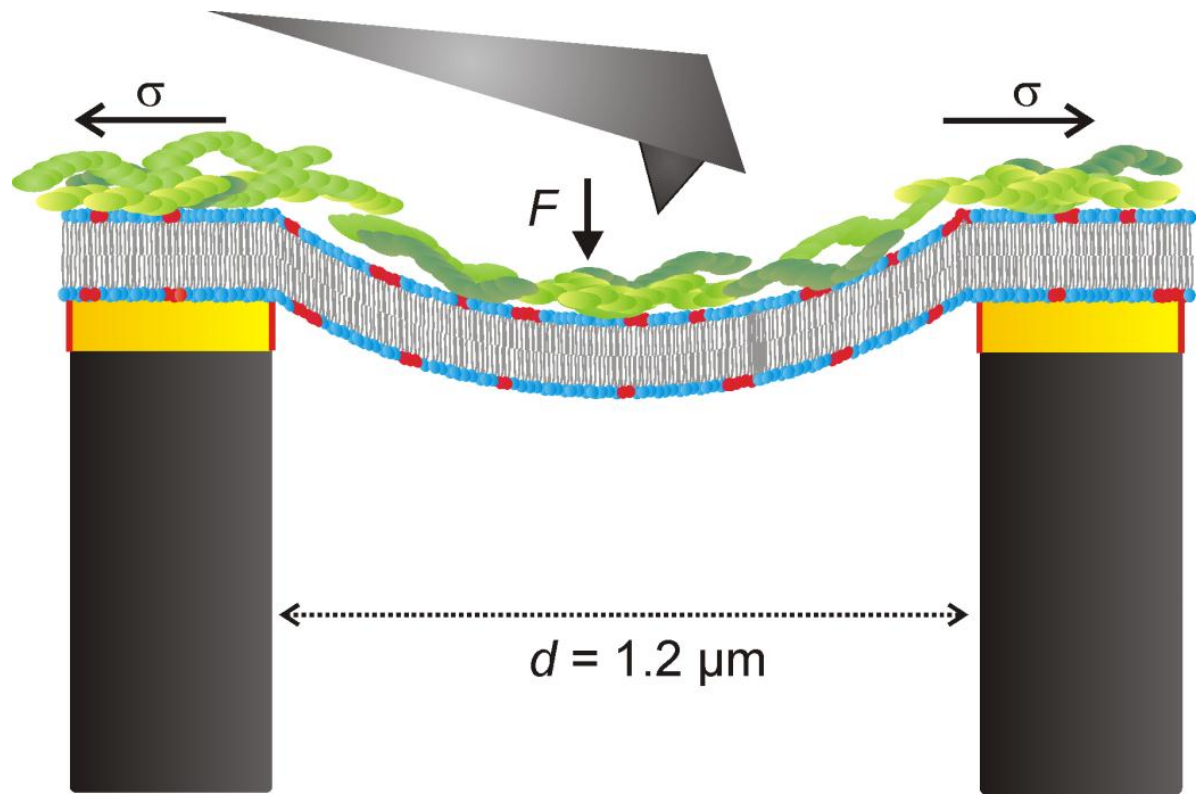

Fig. 8.3 Schematic drawing showing an indentation experiment of a pore spanning membrane composed of DOPC/DOEPC, 8:2 with an attached F-actin network ( $F=$ applied force, $\sigma=$ lateral tension, $d=$ pore diameter).

This assay will in the future allow to systematically modulate the attached F-actin network using artificial and natural cross linking agents and proteins. Thus, the impact of different $\mathrm{ABPs}$ on the membrane mechanics and viscoelastic properties can be determined.

\subsection{Results}

\subsubsection{F-actin Binding Assay on Solid Supported Membranes}

To specifically immobilize filamentous actin (F-actin) on solid supported and pore spanning lipid bilayers, we first aimed to establish a binding assay dealing with the interaction of F-actin with positively charged solid supported membranes. Hence, solid supported membranes composed of the zwitterionic matrix lipid DOPC and the positively charged lipid DOEPC were prepared on either silicon wafers or gold coated 
LaSFN9 glass plates functionalized with octanethiol. Proper vesicle spreading as well as F-actin binding was confirmed by means of the surface plasmon resonance (SPR) technique ${ }^{28}$ that allows to monitor both membrane formation and actin binding in real time, and confocal laser scanning microscopy (CLSM).

In Fig. 8.4 A the kinetics of the spreading process of small unilamellar vesicles (SUVs) composed of pure DOPC (red line) and DOPC/DOEPC, 7:3 (black line) on a gold covered glass plate functionalized with octanethiol in F-buffer $(50 \mathrm{mM} \mathrm{KCl}, 0.2 \mathrm{mM} \mathrm{MgCl}$, $0.1 \mathrm{mM}$ EDTA, $0.1 \mathrm{mM} \mathrm{NaN}_{3}, 20 \mathrm{mM}$ TRIS/HCl, pH 7.4) is shown. After rinsing with buffer the final change of relative reflectivity was rel. refl. $=0.04$, which corresponds to a monolayer thickness of $d=1.6 \mathrm{~nm}$.
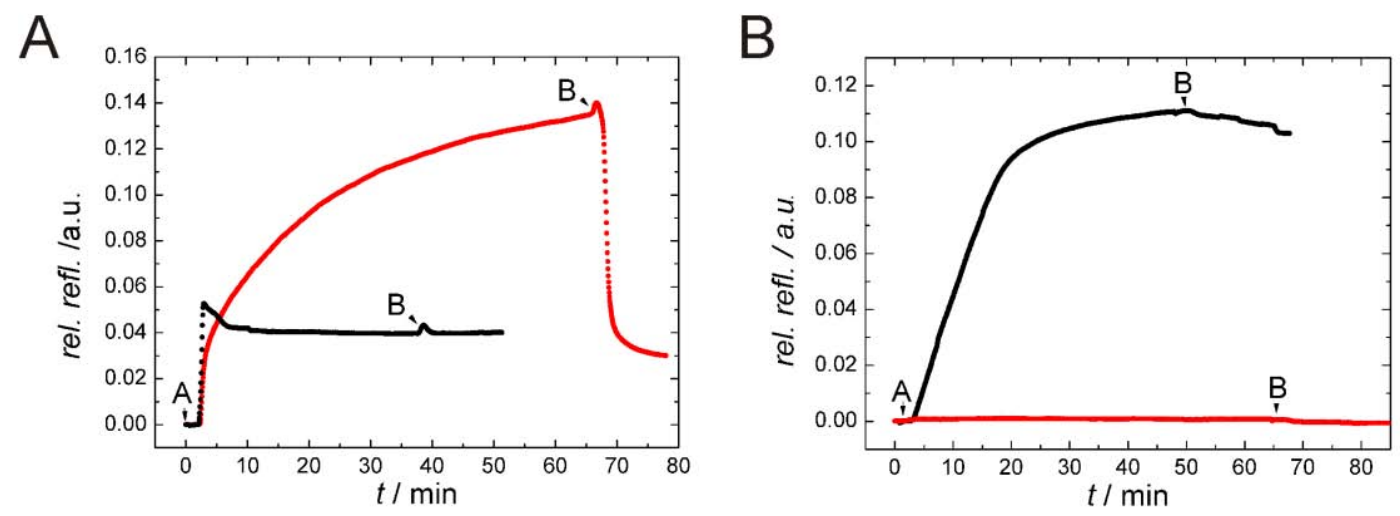

Fig. 8.4 (A) Time course of the relative reflectivity shift after the addition of SUVs (A) composed of pure DOPC (red curve) and DOPC/DOEPC, 7:3 (black curve) and rinsing with F-buffer (50 mM KCl, $0.2 \mathrm{mM}$ $\mathrm{MgCl}_{2}, 0.1 \mathrm{~mm}$ EDTA, $0.1 \mathrm{mM} \mathrm{NaN}_{3}, 20 \mathrm{~mm}$ TRIS/HCl, pH 7.4). (B) Time course of the relative reflectivity shift after the addition of $1 \mu \mathrm{M}$ F-actin (A) to a pure DOPC membrane (red curve) and a DOPC/DOEPC, 7:3 membrane (black curve). B indicates the time point of F-buffer rinsing. ${ }^{28}$

In case of the DOEPC containing membrane (black line), buffer rinsing did not lead to any changes in rel. refl., while buffer rinsing of the pure DOPC membrane (red line) results in huge drop of rel. refl. $=0.1$. This large shift indicates the presence of membrane adhered vesicles atop the DOPC monolayer that could be rinsed of with F-buffer. Subsequent addition of $1 \mu \mathrm{M}$ muscular F-actin, stabilized with an equimolar amount of phalloidin, to the DOPC/DOEPC, 7:3 membrane (Fig. 8.4 B, black line), leads to an in- 
crease of relative reflectivity of rel. refl. $=0.1$, corresponding to a layer thickness of $d=4 \mathrm{~nm}$. Addition of $1 \mu \mathrm{M}$ F-actin to the pure DOPC membrane did not significantly alter the change of relative reflectivity (Fig. 8.4 B, red line). This finding demonstrates that a certain content of DOEPC is required to electrostatically immobilize actin filaments on solid supported membranes.

The dependence of F-actin immobilization on the DOEPC content was studied by means of CLSM. The F-actin binding capability of solid supported membranes composed of DOPC containing $0 \mathrm{~mol} \%, 10 \mathrm{~mol} \%, 20 \mathrm{~mol} \%$, and $30 \mathrm{~mol} \%$ DOEPC was quantitatively accessed.

First, solid supported membranes composed of DOPC and various DOEPC contents doped with $1 \mathrm{~mol} \%$ perylene were prepared on silicon supports. A homogenous fluorescence indicates the successful formation of a defect free lipid bilayer. By FRAP experiments continuous and lateral mobile solid supported membranes $(10-30 \mathrm{~mol} \%$ DOEPC) were proven and a mean diffusion coefficient of $D=(0.8 \pm 0.2) \mu \mathrm{m}^{2} \cdot \mathrm{s}^{-1}$ with an immobile fraction of $(8 \pm 4) \%$ was determined $(n=33)$. The obtained diffusion coefficient is similar to those found in the literature for pure DOPC membranes, but slightly lower than the average values $\left(2-4 \mu \mathrm{m}^{2} \cdot \mathrm{s}^{-1}\right) \cdot{ }^{29-31}$ Also, the immobile fraction is larger compared to the almost $100 \%$ mobility of solid supported bilayers reported for pure DOPC membranes. Both findings might be due to an interaction of the positively charged DOEPC lipids with the hydrophilic silicon wafer. A dependence of the diffusion coefficient and the immobile fraction on the DOEPC content could thus be assumed. However, this dependency was not observed within the conducted experiments.

F-actin, labeled and stabilized with AlexaFluor488 phalloidin, was then incubated overnight at $4{ }^{\circ} \mathrm{C}$ with a concentration of $\sim 1 \mu \mathrm{M}$ in F-buffer. After the samples were thoroughly rinsed with pure F-buffer to remove unspecifically bound actin filaments, they were imaged by means of a confocal laser scanning microscope in order to visualize the amount of bound F-actin. Characteristic fluorescence microscopy images of F- 
actin bound to solid supported DOPC membranes with various DOEPC content (0-30 mol\%) are depicted in Fig. 8.5.
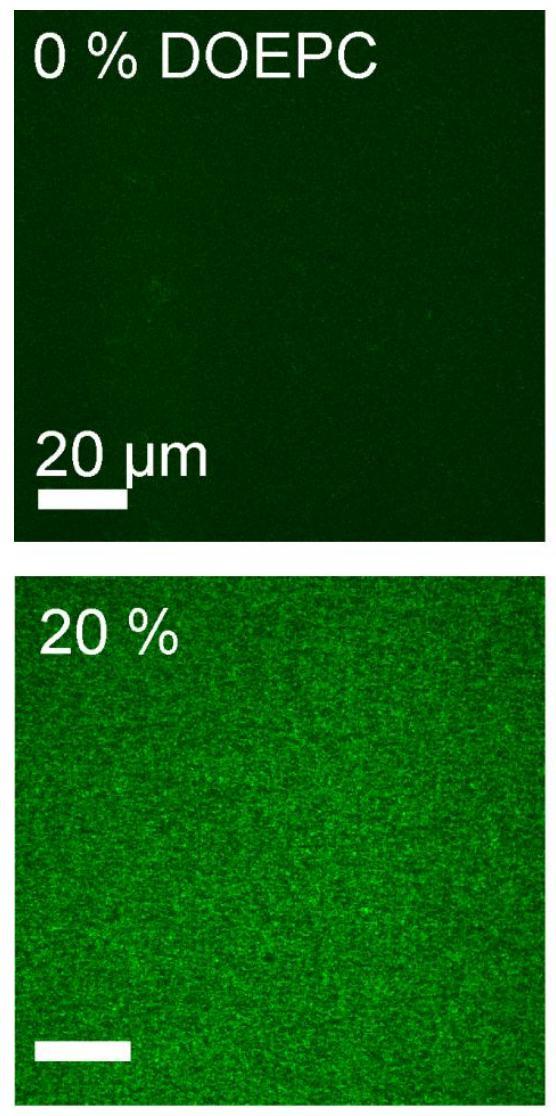
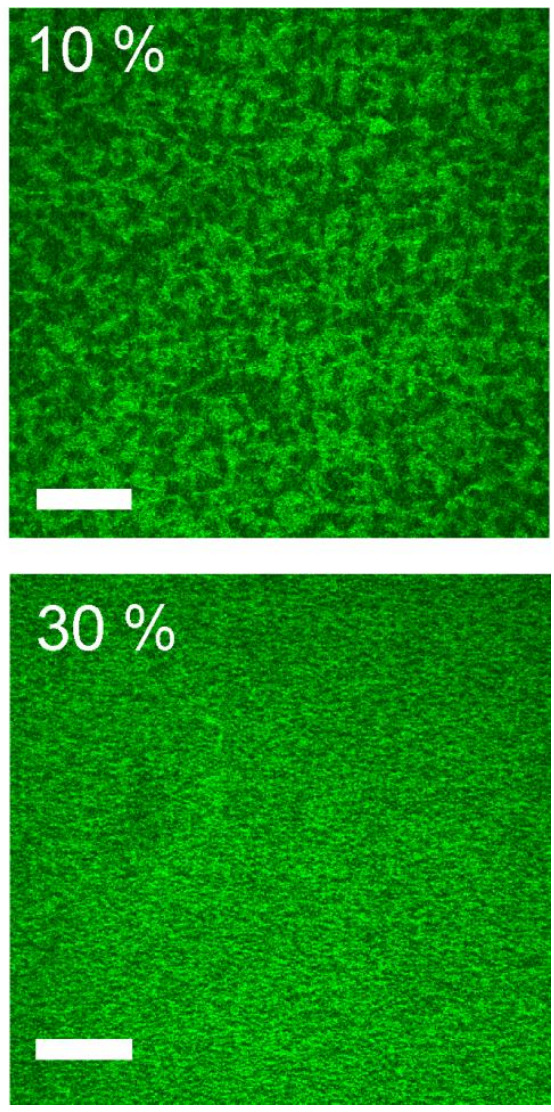

Fig. 8.5 Fluorescence microscopy images showing AlexaFluor488 phalloidin labeled F-actin bound to the surface of DOPC containing solid supported membranes with various DOEPC contents (10-30 mol\%).

While only the background fluorescence and no green AlexaFluor488 phalloidin labeled F-actin can be visualized atop the perylene doped pure DOPC membrane, few patches of F-actin are bound on DOPC membranes with $10 \mathrm{~mol} \%$ DOEPC. On membranes with $20 \mathrm{~mol} \%$ and $30 \mathrm{~mol} \%$ DOEPC an almost complete F-actin surface coverage was imaged. To gather quantitative information about the F-actin membrane coverage as a function of DOEPC content, a pixel analysis was performed (ImageJ) and the F-actin area fraction of each fluorescence image was determined. Based on this approach, an F-actin area fraction of $(7 \pm 3) \%(n=8)$ was determined for pure DOPC 
membranes, $(30 \pm 8) \%(n=20)$ for membranes with $10 \mathrm{~mol} \%$ DOEPC, $(46 \pm 4) \%(n=18)$ for membranes containing $20 \mathrm{~mol} \%$ DOEPC and $(52 \pm 5) \%(\mathrm{n}=26)$ for $30 \mathrm{~mol} \%$ DOEPC containing membranes. The mean area fraction of F-actin dependent on the DOEPC content is depicted in Fig. 8.6.

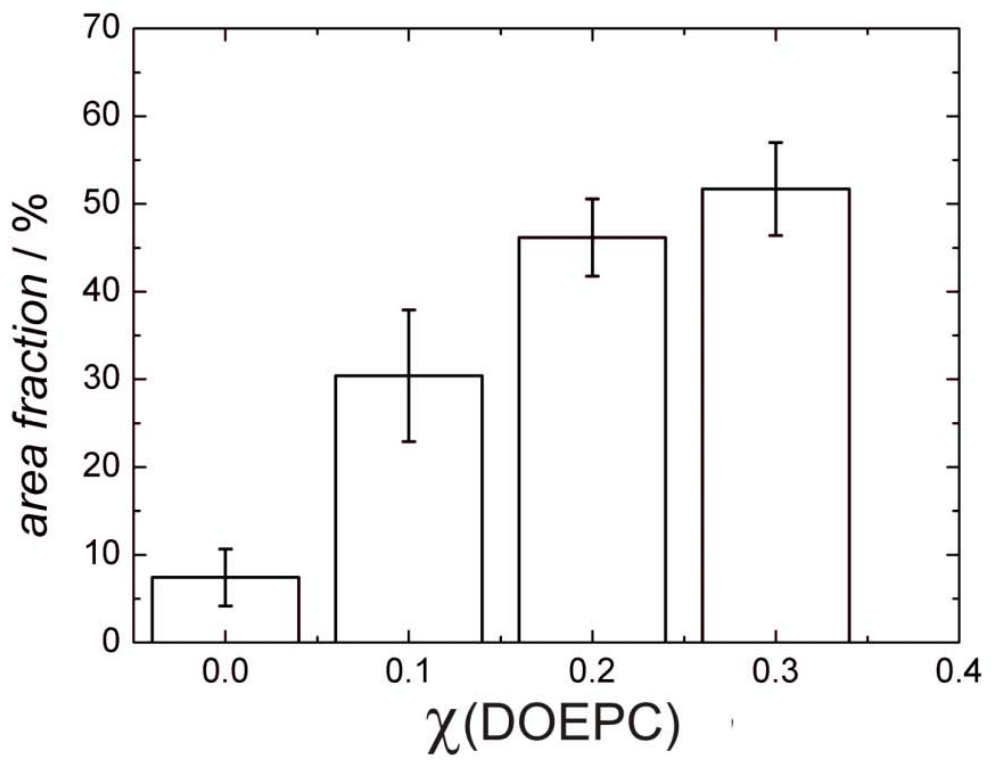

Fig. 8.6 Quantification of the F-actin membrane surface coverage as a function of the DOEPC fraction (X).

These results show that a sufficiently dense F-actin network was obtained between 20 and $30 \mathrm{~mol} \%$ of the positively charged lipid DOEPC into the membrane.

\subsubsection{F-actin Binding on Pore Suspending Membranes}

Pore spanning membranes composed of DOPC doped with $20 \mathrm{~mol} \%$ DOEPC and $1 \mathrm{~mol} \%$ perylene were prepared on porous silicon nitride surfaces (pore diameter $d=1.2 \mu \mathrm{m}$ ) by spreading giant unilamellar vesicles (GUVs, diameter $d=5-20 \mu \mathrm{m}$ ). Owing to the F-actin surface coverage analysis performed on solid supported membranes, 
$20 \mathrm{~mol} \%$ of the positively charged DOEPC content was chosen to guarantee a sufficient surface coverage of the pore spanning membranes with F-actin.

The porous substrates were first coated with a thin layer of gold and functionalized with mercaptoethanol (see chapter 3.1.2, page 29) to generate a hydrophilic surface that facilitates GUV rupturing. ${ }^{27}$ After adding $30 \mu \mathrm{l}$ of GUVs $(0.15 \mathrm{mg} / \mathrm{ml}$ in $300 \mathrm{~mm}$ sucrose solution) to the freshly functionalized porous substrate immersed in PBS-buffer (2.7 $\mathrm{mM} \mathrm{KCl}, 136.9 \mathrm{mM} \mathrm{NaCl}, 1.5 \mathrm{mM} \mathrm{KH}_{2} \mathrm{PO}_{4}, 8.1 \mathrm{mM} \mathrm{Na}_{2} \mathrm{HPO}_{4}$, $\mathrm{pH}$ 7,4), the samples were incubated for $1 \mathrm{~h}$ at room temperature. They were then carefully rinsed with buffer to remove non spread GUVs adhered on the surface. Qualitative investigation of the lateral membrane mobility by means of FRAP experiments revealed an appropriate membrane quality, indicated by a complete fluorescence recovery within few seconds. Fig. 8.7 A shows the perylene fluorescence of a typically shaped membrane patch obtained after GUV spreading.
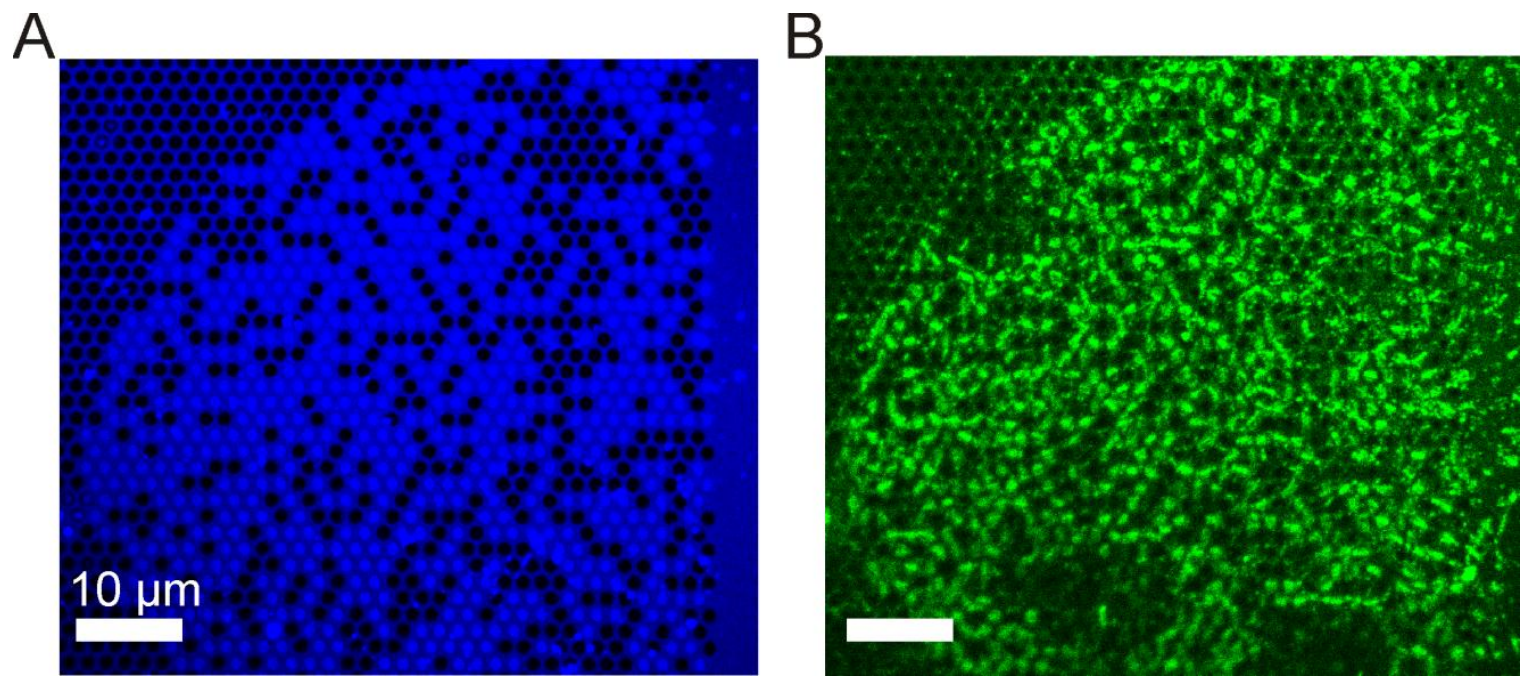

Fig. 8.7 Fluorescence microscopy images of pore spanning membranes composed of DOPC/DOEPC/perylene, 79:20:1 (A) and corresponding fluorescence of the attached AlexaFluor488 labeled F-actin network (B).

Unfortunately the long term stability of the F-actin covered membranes was reduced compared to PSMs composed of other lipids. ${ }^{27,25}$ Imaging the same membrane patches 
after $12 \mathrm{~h}$, it became obvious that membrane rupturing events caused the originination of non covered porous areas. In Fig. 8.7 B the fluorescence of AlexaFluore488 phalloidin labeled actin filaments bound on the same membrane patch is shown. It is obvious that there are many non spanned pores within the membrane patch and that these areas are non covered with actin filaments. Indeed, the membrane fluorescence perfectly overlaps with the location of F-actin. Confocal laser scanning microscopy studies were not only utilized to confirm the membranes quality and presence of filamentous actin, but also to be able to later localize certain membrane patches. Owing to the unique pattern of the fluXXion coordinate system, correct positioning of the AFM cantilever tip atop the desired patch could be facilitated.

\subsubsection{Force Spectroscopic Characterization of F-actin Decorated Pore Spanning Membranes}

Membrane patches with and without an attached F-actin network were imaged by means of force volume mapping using the atomic force microscope (AFM). Force maps, where every pixel represents a force distance curve, were recorded. The height information of a force map, represented by a brightness scale, correlates with the indentation depth of the cantilever before a certain force (trigger force) is reached. Hence, membrane covered pores appear brighter than non covered pores, because the cantilever tip travels a smaller distance until it reaches the preset trigger force (300 or $500 \mathrm{pN}$ ). A characteristic force map of pore spanning membranes composed of DOPC/DOEPC/perylene, 79:20:1 are depicted in Fig. 8.8 A. together with the corresponding indentation curves that are plotted as force distance curves. 
A
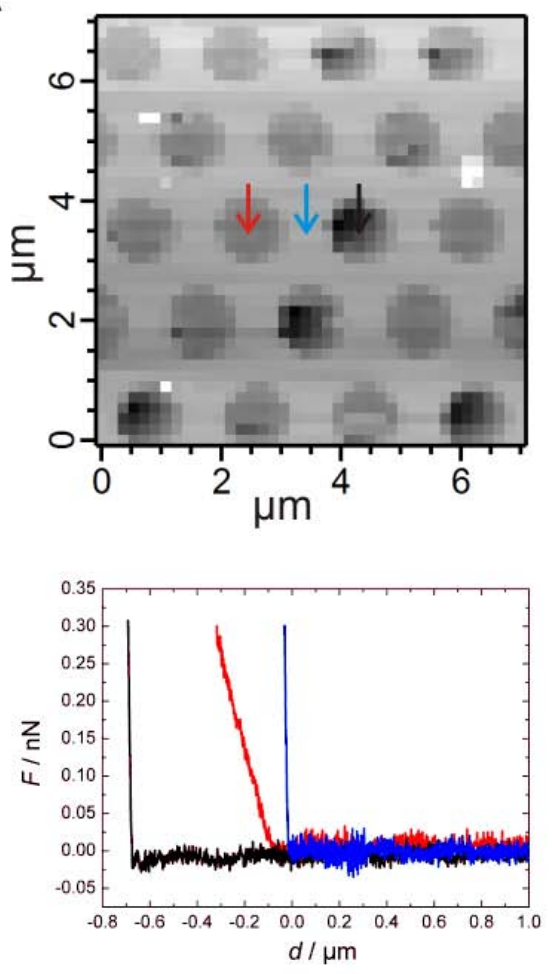

B
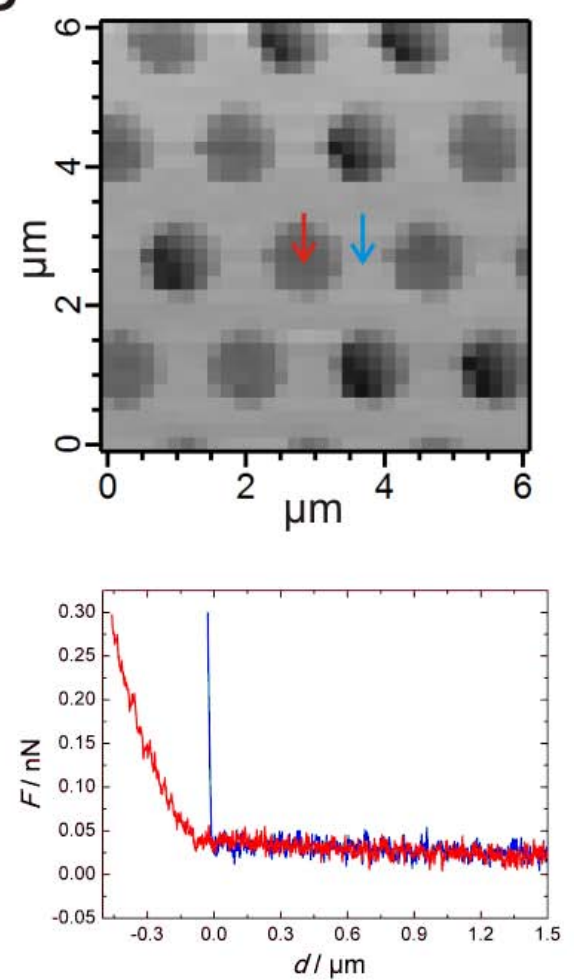

Fig. 8.8 Force maps and indentation curves of (A) pore spanning membranes composed of DOPC/DOEPC/perylene, 79:20:1 and (B) of F-actin decorated pore spanning membranes composed of DOPC/DOEPC/perylene, 79:20:1. The force indentation curves obtained from locations referenced by arrows of corresponding color.

While the cantilever travels approximately $700 \mathrm{~nm}$ inside an empty pore, until it reaches the trigger force, the contact point of the pore spanning membrane is nearly identical with that of the pore rims. This finding demonstrates that the membranes do not significantly invaginate into the pores. Both the force distance curve obtained from the pore rim (blue) and from an uncovered pore (black) have very steep slopes, indicative of a hard substrate repulsion. However, the slope of the force indentation curve for the PSM is less steep. An apparent spring constant ( $\left.k_{\mathrm{app}}\right)$ of the pore spanning membrane, as an indicator of the membrane stiffness, can be obtained by a linear regression of the indentation curve. A representative force map together with the corresponding indentation curves obtained from pore spanning DOEPC containing membranes with bound F-actin is shown in Fig. 8.8 B. The mean apparent spring constant of pore spanning 
membrane without bound F-actin was determined to $k_{\mathrm{app}}=(1.7 \pm 0.3) \mathrm{mN} / \mathrm{m}, \mathrm{n}=223$, while pore spanning membranes covered with F-actin exhibit a mean spring constant of $k_{\text {app }}=(2.2 \pm 0.6) \mathrm{mN} / \mathrm{m}, \mathrm{n}=98$. A histogram that was fit with a Gaussian function shows the frequency of the apparent spring constant of DOPC/DOEPC, 8:2 pore spanning membranes (non filled bars) and pore spanning membranes with attached actin filaments (grey bars) are presented in Fig. 8.9.

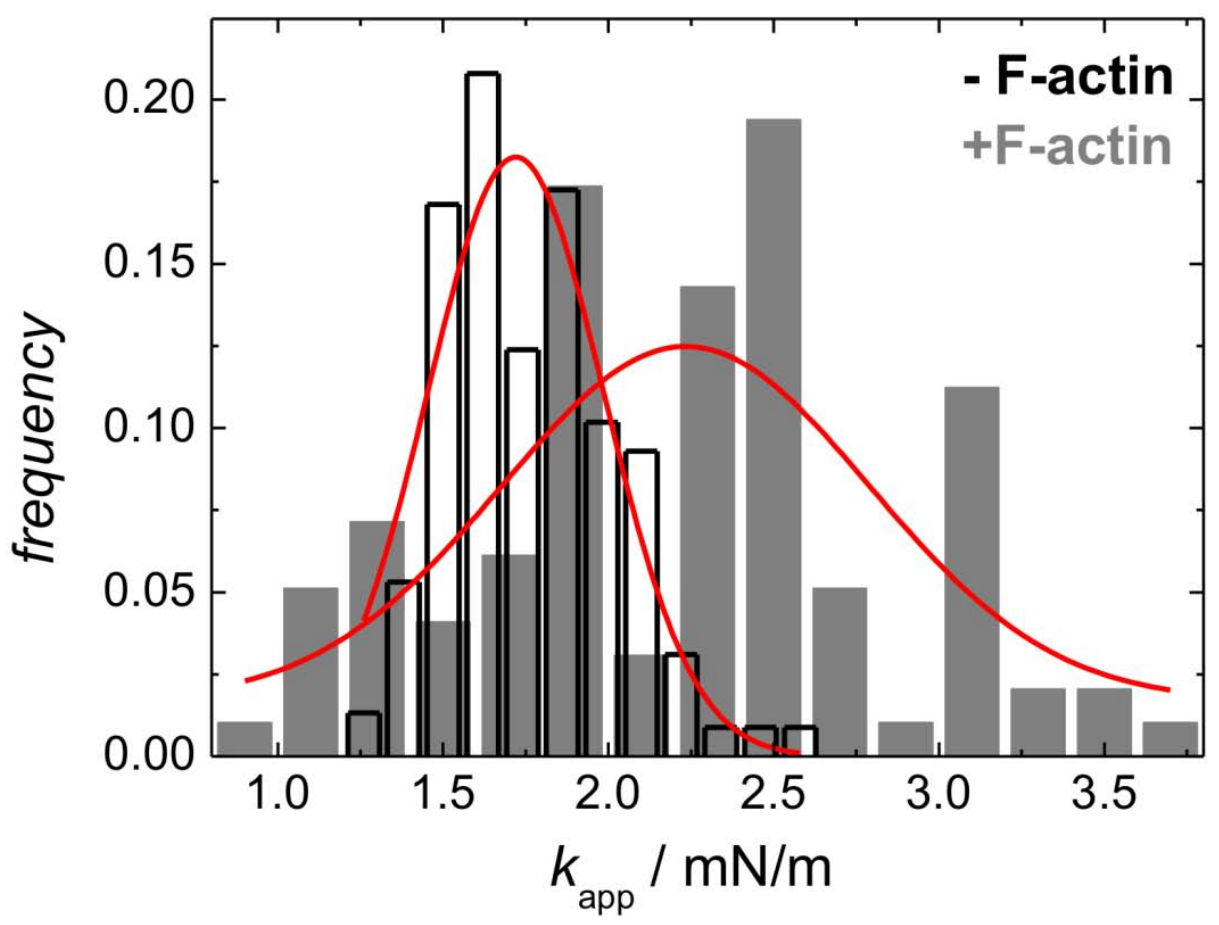

Fig. 8.9 Histogram fit with a Gaussian function shows the frequency of apparent spring constants $\left(k_{\text {app }}\right)$ of pore spanning membranes composed of DOPC/DOEPC/perylene, 79:20:1 without (non filled bars, $\left.<k_{\text {app }}>=(1.7 \pm 0.3) \mathrm{mN} / \mathrm{m}, \mathrm{n}=223\right)$ and with an attached F-actin network (grey bars, $<k_{\text {app }}>=(2.2 \pm 0.6)$ $\mathrm{mN} / \mathrm{m}, \mathrm{n}=98)$.

The histogram reveals that, although the mean values of the apparent spring constants are very similar, their distribution is not. While there is a quite narrow distribution in case of the non actin covered pore spanning membranes with a maximum at $\sim 1 \mathrm{mN} / \mathrm{m}$, the apparent spring constant of pore spanning membranes with F-actin bound is broadly distributed with a maximum that was shifted to higher values of $k_{\text {app. }}$ This 
broad distribution of kapp shows the heterogeneity of F-actin covered pore spanning membranes.

Characteristic indentation and retraction curves obtained from pore spanning membranes covered with F-actin are depicted in Fig. $8.10 \mathrm{C}$ together with the indentation and retraction curves of a non actin covered pore spanning membrane (Fig. 8.10 B). In all cases, the response of the non actin covered pore spanning membranes was fully elastic, indicated by the lack of a hysteresis. For F-actin covered pore spanning membranes, however, a hysterises between the retraction and indentation curve was observed. The area between the indentation and retraction curve equals to the energy dissipation during indentation. A histogram that depicts the frequency of energies determined for pore spanning membranes without F-actin immobilized on the membrane surface (hollow bars) and with F-actin bound to the membrane (grey bars) is shown in Fig. 8.10 A.
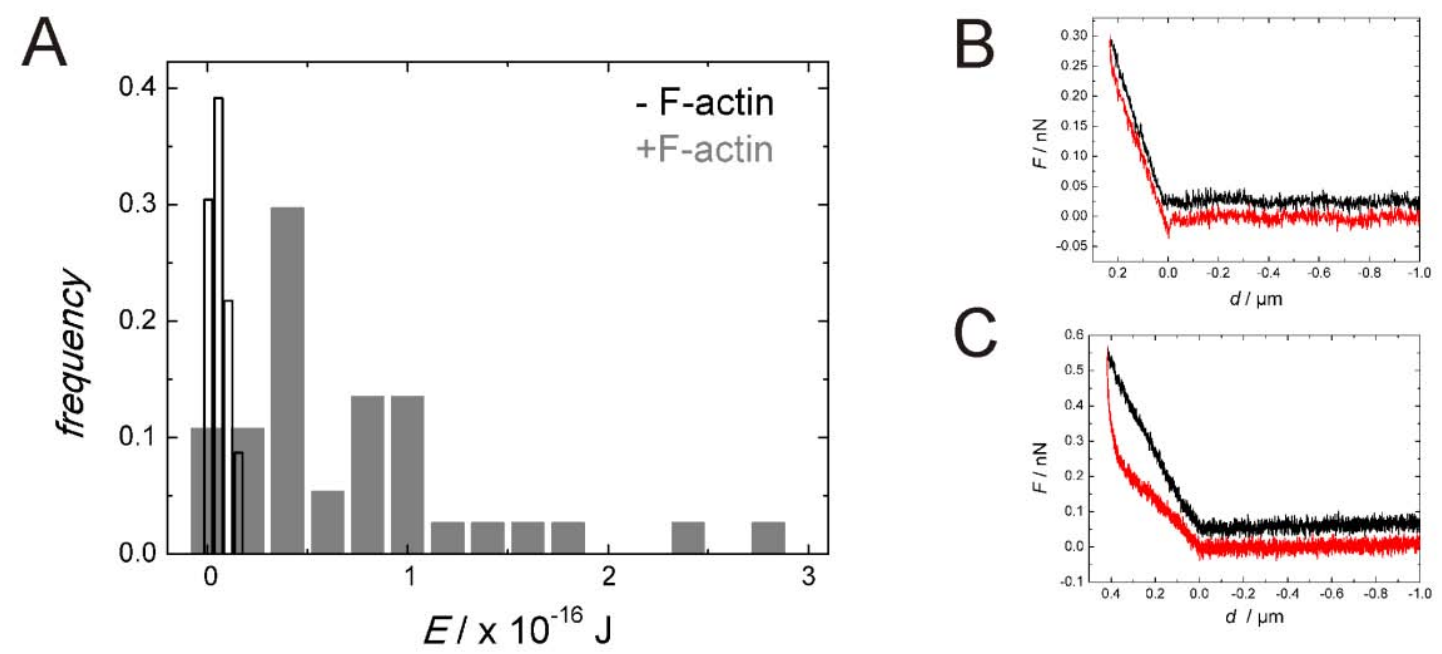

Fig. 8.10 (A) Histogram showing the frequency of energies of a pore spanning membrane without (non filled bars, $\left.E=(0.6 \pm 0.4) \cdot 10^{-17} \mathrm{~J}, \mathrm{n}=22\right)$ and with (grey bars, $E=(7 \pm 6) \cdot 10^{-17} \mathrm{~J}, \mathrm{n}=37$ ) an attached F-actin network. Indentation (black line) and retraction (red line) curves of (B) membranes with bound F-actin and without $(\mathbf{C})$. Energies where determined as the area between indentation and retraction curve. 
The energy distribution is very narrow and close to zero, highlighting the non observed hysteresis for non F-actin covered pore spanning membranes. The energies determined for F-actin covered pores spanning membrane, however, are more broadly distributed and larger, as most of the indention events showed a hysteresis, which is attributed to the viscoelastic character of F-actin decorated membranes.

\subsection{Discussion}

The mechanical and viscoelastic properties of F-actin networks have been extensively studied in the past, examining in vitro polymerized actin gels or cells that were selectively manipulated with certain drugs to deliberately affect the cytoskeleton. ${ }^{1,9,13,17,32-34}$ Here, we extended an earlier developed assay based on pore spanning membranes $27,26,25,24$, which allows us to investigate the influence of an attached, in vitro polymerized F-actin network on the mechanical membrane properties. PSMs generated by spreading giant unilamellar vesicles composed of DOPC/DOEPC/perylene, 79:20:1 on gold coated silicon nitride porous substrates that were functionalized with mercaptoethanol. The positive membrane charges, realized by introducing the positively charged lipid DOEPC, enabled us to specifically immobilize F-actin on the membrane surface via electrostatic interactions. This assay allows us to systematically examine the impact of F-actin crossklinker agents and proteins on the membrane mechanics and viscoelastic properties.

First, the specific of F-actin binding to the positively charged lipid DOEPC was investigated by means of the optical biosensor technique surface plasmon resonance (SPR). Spreading of pure DOPC SUVs as well as SUVs that were doped with $30 \mathrm{~mol} \%$ DOEPC was performed on gold plates that had been functionalized with octanethiol. The spreading kinetic of the DOPC membrane, indicates vesicles adsorption atop the monolayer surface, as a steady increase of relative reflectivity was monitored, while in case of the DOPC membranes a baseline was reached shortly after vesicles addition. Differ- 
ent spreading mechanisms for positively and neutrally charged SUV's were reported in 2008 by Wikström et al.. They monitored formation of DOEPC and POPC lipid bilayers by means of the QCM-D technique on AT-cut quartz crystals coated with $\mathrm{SiO}_{2}{ }^{35} \mathrm{The}^{2}$ spreading kinetics of vesicles on hydrophobic surface ${ }^{36}$, however, differ from those on hydrophilic surfaces, so that an accurate comparison can not be conducted. Rinsing with F-buffer removed most of the vesicles that were adhered on the DOPC membrane, so that both membranes exhibited final thicknesses of $d=1.6 \mathrm{~nm}$. This value is slightly lower than expected for lipid monolayers $(d=2 \mathrm{~nm}) \cdot{ }^{37,38}$ Nevertheless, an appropriate membrane quality was guaranteed, as the presence of large defects was ruled out by the fact that no unspecific binding of F-actin occurred on the pure DOPC membrane. As F-actin strongly and irreversibly adheres on plain octanethiol surfaces, the DOPC membrane must have been rather defect-free. While no F-actin binding was observed on membranes lacking DOEPC, a $30 \mathrm{~mol} \%$ DOEPC content resulted in a F-actin thickness of $d=4 \mathrm{~nm}$. As each actin filament has a mean diameter of $7 \mathrm{~nm}$, a filament film thickness of $7 \mathrm{~nm}$ could principally be obtained assuming a $100 \%$ surface coverage. In our work, however, the formation of an actin filament meshwork atop the membrane resulted in a surface coverage of $57 \%$. The interaction of F-actin, which harbors four negative charges per monomer at $\mathrm{pH} 7.4$ (pI 5.6), with positively charged compounds has been studied previously. Although Gicquaud et al. even reported an interaction of F-actin with neutral lipids in the presence of millimolar $\mathrm{Mg}^{2+}$ concentration ( 1-2 $\mathrm{mM}$ $\left.\mathrm{Mg}^{2+}\right)^{39}$, this finding could not be confirmed in this work, as no F-actin binding to pure DOPC membranes was observed. Apparently the concentration of $0.2 \mathrm{mM} \mathrm{Mg}^{2+}$ used in our work, was not sufficient to mediate F-actin binding to neural membranes, since neither CLSM images nor OWS experiments showed F-actin binding to pure DOPC membranes. Demé et al., who investigated the adsorption of F-actin to positively charged lipid monolayers by means of neutron reflectivity, calculated a maximal packing volume of $37 \%$, considering actin filaments to pack as cylindrical rods, taking the $\alpha$-helical appearance of F-actin into account. They reported a volume fractions that corresponds to $68-78 \%$ of the theoretical packing volume depending on the ionic 
strength. ${ }^{40}$ Barfoot et al. only detected a F-actin thickness $0.4 \mathrm{~nm}$ change, which corresponds to $6 \%$ surface coverage. By means of the SPR technique, they measures binding of actin filaments to a membrane containing ponticulin, a natural F-actin receptor protein. ${ }^{41}$ The discrepancy between the surface coverage of $57 \%$ determined in this work and the maximal possible packing volume (37\%) can be explained by filament stacking, possibly mediated by positively charged ions. However, the main reason for this deviation might be the $x-y$-resolution limit of the CLSM $(d \approx 200 \mathrm{~nm})$ due to Abbe's law has to be taken into account. This leads to an overestimation of the apparent surface coverage.

The fluorescence microscopy assay that examines the F-actin surface coverage as a function of DOEPC content further confirms the specific DOEPC/F-actin binding, as no fluorescence signal could be detected on pure DOPC membranes. Furthermore, the DOEPC content necessary to completely cover the solid supported membrane with actin filaments was confined to $20-30 \mathrm{~mol} \%$ (see Fig. 8.5 and Fig. 8.6). Thus, GUVs containing DOPC with a $20 \mathrm{~mol} \%$ DOEPC were prepared to produce pore spanning membranes. GUVs with sizes ranging from $d=5-20 \mu \mathrm{m}$ were obtained in this work. These GUV sizes are rather small compared with vesicles composed of uncharged lipids obtained by the electroformation technique, where GUVs with diameters of $\sim 100 \mu \mathrm{m}$ could be prepared. ${ }^{42}$ The relatively small GUV size obtained in this work might be caused by the amount of positively charged lipid. Rodriguez et al. demonstrated that with negatively charged lipids (here: DOPS) with a content higher than $20 \%$, GUV formation by means of the electroformation method could not be achieved, while with the gentle hydration method this content could be increased up to $40 \%{ }^{43}$

The membranes patches that were formed after spreading of the GUVs exhibited a homogenous fluorescence and a full fluorescence recovery after bleaching, demonstrating the formation of a pore spanning lipid bilayer. The contact point of the AFM cantilever tip is identical to the one of the pore rim. ${ }^{27}$ This implies that the lipid bilayer spans the porous region without invaginating the pore interior. This finding is typical of PSM formed on hydrophilically functionalized pores, while hybrid PSM cover the entire 
functionalized gold surface to gain maximal adhesion energy, even though it implies an increased membrane curvature. ${ }^{25}$ This difference between hybrid and non hybrid PSMs is depicted in Fig. 8.11.

A

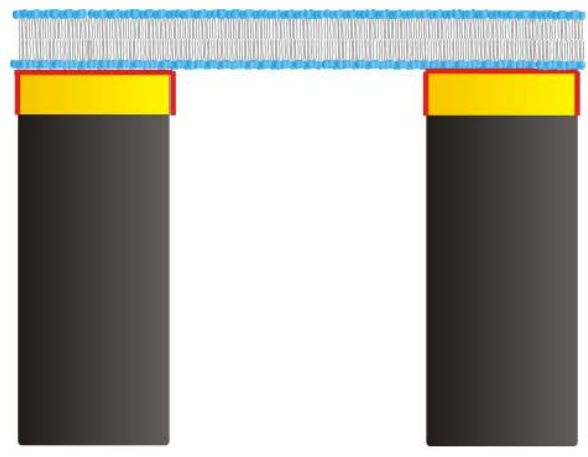

B

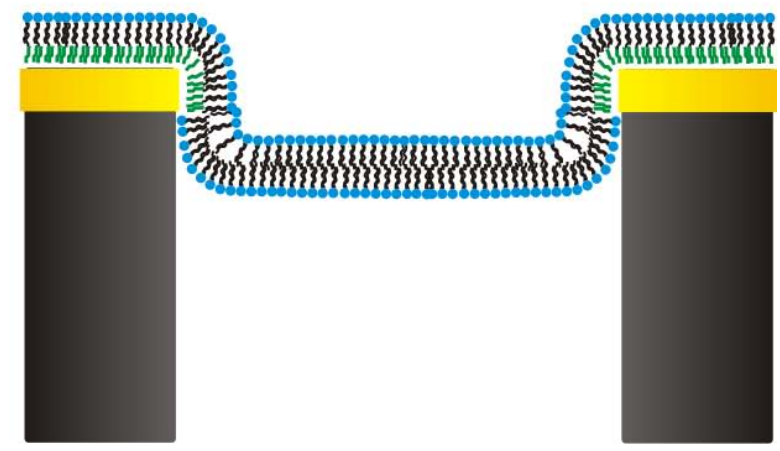

Fig. 8.11 (A) Pore spanning membrane formed on hypdrophilically functionalizes pore rims and (B) hybrid pore spanning membrane on hydrophobically functionalized porous substrates.

The apparent spring constant ( $\left.k_{\text {app }}\right)$ of the DOPC/DOEPC, 8:2 pore spanning membranes formed on pores functionalized with mercaptoethanol was determined to $k_{\text {app }}=(1.7 \pm 0.3) \mathrm{mN} / \mathrm{m}(\mathrm{n}=223)$ by linear regression of the indentation curve. This value is in very good agreement with the apparent spring constant of pure DOPC membranes $k_{\text {app }}=(1.42 \pm 0.02) \mathrm{mN} / \mathrm{m}$ measured under similar conditions by Kocun et al. in $2011 .{ }^{27}$ It is not surprising that the apparent spring constant, as an indicator for the lateral membranes tension ( $\sigma$, see Fig. 8.3), of hybrid PSMs is about 10fold higher, due to the pre-stress of the hybrid membrane. Differences in membrane tension were also reported for different lipid mixtures and pore radii. ${ }^{26,25}$ The lateral tension is mainly determined by the bilayer adhesion to the pore rim. In fact, of all three mechanical contributions (lateral tension, bending, stretching) that may be considered regarding PSMs, the lateral tension $\sigma$ determines the mechanical properties of PSMs. In fact, the influence of membrane bending is neglidably in this case, as the pore radius ( $\left.R_{\text {Pore }}\right)$ is sufficiently high and the radius of the cantilever tip $\left(R_{\mathrm{Tip}}\right)$ as well as the trigger force is ade- 
quately small. Stretching of the membrane can also be neglected, as PSMs would rupture, if their area is increased over $5 \%$ upon indentation. ${ }^{25,24}$ Following theses assumptions, the lateral tension $\sigma$ can be calculated using the following relationship (eq. 8.1) ${ }^{27}$ :

$$
x=\frac{F(x)}{4 \pi \sigma}\left[1-\ln \left(\frac{F(x) R_{\text {Tip }}}{2 \pi \sigma R_{\text {Pore }}^{2}}\right)\right],
$$

with $F(x)$ being the force upon indentation and $x$ being the indentation depth.

Thus, a membrane tension of $\sigma=1.22 \mathrm{mN} / \mathrm{m}$ was calculated for the non F-actin covered PSMs and of $\sigma=1.64 \mathrm{mN} / \mathrm{m}$ for the PSMs that were covered with a F-actin network, respectively

Regarding the F-actin binding on the pore spanning membranes, it became obvious that not all membranes patches were covered with the same amount of actin filaments (compare Fig. 8.7.) In some cases only few filaments were adsorbed, on some patches no attached F-actin network could be observed. This was most likely due to a reduced DOEPC content of the regarding membrane patch, possibly caused by a yet heterogeneous DOEPC content in the GUVs. Larson et al. investigated the distribution of two fluorescently labeled lipids within GUVs. They found that the molar ratio of the two fluorophores in different GUVs differed up to a factor of seven. ${ }^{4}$

The tension of the membrane $\sigma$ can be linearly correlated with the apparent spring constant found for PSMs with an attached F-actin network, which was determined to be $k_{\mathrm{app}}=(2.2 \pm 0.6) \mathrm{mN} / \mathrm{m}, \mathrm{n}=98$. This value does not significantly differ from the one of the pure pore spanning membrane, but the distribution (see histogram, Fig. 8.9) is clearly higher, ranging from $0.9-3.7 \mathrm{mN} / \mathrm{m}$. This result is supported by force indentation experiments of apical membrane fragments prepared from MDCK II cells on porous silicon nitride substrates performed by Fine et al.. Here, depolymerization or dis- 
solution of F-actin by DNAse I and gelsolin did not alter the apparent spring constant compared to the one of native cell fragments. ${ }^{45}$

Other than with experiments that measure the elastic behavior of F-actin gels in solution, experiments performed on cells or pore spanning membranes would not directly allow to determine the elastic modulus $(E)$ of actin filaments. As the elastic modulus of F-actin $(E \approx 1-10 \mathrm{kPa})^{8,13,12}$ is in the same range as the elastic modulus of different cell types $(E \approx 1-50 \mathrm{kPa})^{21}$ and even 1000 fold lower than the one determined for polymer pore spanning membranes $(E \approx 1-50 \mathrm{MPa})^{46}$, it cannot be diretly determined utilizing cellular or membrane systems. Instead, the impact of the F-actin network or its cross linking degree on the membrane mechanics can be determined. The main drawback of using cells to determine the influence of the cortical F-actin network on the membrane mechanics, is the indefinable contribution of the cellular Newtonian liquid on their viscoelastic response. Also, results may be difficult to interpret due to manifold complex cytoskeletal components that contribute to the cellular mechanical properties. To really be able to distinguish the contribution of the actin network on membrane tension and mechanics, it is of importance to create a system that or allow to control these interfering factors.

To overcome these drawbacks, the development of appropriate model systems is mandatory with reduced variable parameters, where the impact of the cytoskeletal proteins on the membrane mechanics can be investigated independently from each other. The reconstitution and organization of F-actin networks inside giant unilamellar vesicles have been studied in the recent past. ${ }^{47-49}$ While Cortese et al. found that vesicle deformation can be triggered by the actin filament $\operatorname{size}^{49}$, Limozin et al. showed that the cortical layer thickness inside the vesicle can be governed by the size of the vesicle ${ }^{48}$. Helfer et al. take one step further by studying the viscoelastic properties of GUVs that were coated with F-actin by means of optical tweezer microrheology experiments. ${ }^{50}$

As for the experiments performed in this work, a planar membrane system based on PSMs with an attached F-actin network was utilized to study the influence of F-actin on 
the membrane mechanics and viscoelastic properties. Here, we observed a hysteresis between the indentation and the relaxation curve at most of the force distance curves. The presence of a hysteresis indicates the dissipation of energy during the indentation/retraction cycle due to viscoelastic properties inherited by the material. This phenomenon can be observed in experiments with living cells, cellular membrane fragments and artificial polymer systems. ${ }^{51,23,46}$ Nevertheless, comparing the hysteresis observed in our work with the one obtained from cellular experiments, there are distinct differences between the shape and the velocity dependency. First, in cell experiments, the hysteresis increases as a function of probe velocity ${ }^{51}$, while in our experiments it seems to be vice versa, although more experimental data has to gathered to confirm this finding. Our velocity dependence is in accordance with the one determined for polymer pore spanning membranes by Kocun et al. in $2011 .{ }^{27}$ Second, the indentation curve is rather linear, while a significant curvature was mainly observed in case of the retraction curve. A sufficient curvature however, is mandatory to determine the elastic modulus $E$ of the PSM. ${ }^{46}$ This finding is inconsistent with both cell experiments and experiments performed on polymer pore spanning membranes. ${ }^{46}$

\subsection{Outlook}

To completely determine the dependence of the hysteresis on the of F-actin membrane coverage and probe velocity more experiments have to be conducted to obtain relevant statistical information.

Experiments with artificial F-actin cross linking agents (i.e. glutaraldehyde, polyformaldehyde) or proteins (i.e. filamin, $\alpha$-actinin) could be performed to elucidate and speficically assign the impact of theses crosslinkers to the membrane tensions and viscoelastic behavior. Proteins that link actin filaments to the pores spanning membrane such as. ezrin, would elucidate, how a connetion of the cortical cytoskeleton to the membrane infleuence the membrane mechanics. 
256 I Mechanical Characterization of F-actin on PSMs

\subsection{Co-workers}

Schwamborn, Miriam; Mey, Ingo 


\subsection{References}

[1] Fletcher, D. A., and Mullins, R. D. (2010) Cell mechanics and the cytoskeleton. Nature 463, 485-492.

[2] Voet, D., and Voet, J. G. (2011) Biochemistry. 4th ed., John Wiley \& Sons, Hoboken, NJ.

[3] Fluorescent cells. http://rsb.info.nih.gov/ij/images/ (2012) cited on 2012 Dec 9.

[4] Wickstead, B., and Gull, K. (2011) Evolution: The evolution of the cytoskeleton. J. Cell. Biol. 194, 513-525.

[5] Köster, S., and Pfohl, T. (2009) An in vitro model system for cytoskeletal confinement. Cell Motil. Cytoskel. 66, 771-776.

[6] Podolski, J. L., and Steck, T. L. (1990) Length distribution of F-actin in Dictyostelium discoideum. J. Biol. Chem. 265, 1312-1318.

[7] Yogurtcu, O. N., Kim, J. S., and Sun, S. X. (2012) A Mechanochemical Model of Actin Filaments. Biophys. J. 103, 719-727.

[8] Pujol, T., Du Roure, O., Fermigier, M., and Heuvingh, J. (2012) Impact of branch ing on the elasticity of actin networks. Proc. Natl. Acad. Sci. U.S.A 109, 1036410369.

[9] Gardel, M. L. (2004) Elastic Behavior of Cross-Linked and Bundled Actin Networks. Science 304, 1301-1305.

[10] Lieleg, O., Claessens, M. M. A. E., and Bausch, A. R. (2010) Structure and dynamics of cross-linked actin networks. Soft Matter 6, 218.

[11] Janmey, P. A., Hvidt, S., Käs, J., Lerche, D., Maggs, A., Sackmann, E., Schliwa, M., and Stossel, T. P. (1994) The mechanical properties of actin gels. Elastic modulus and filament motions. J. Biol. Chem. 269, 32503-32513. 
[12] Chaudhuri, O., Parekh, S. H., and Fletcher, D. A. (2007) Reversible stress softening of actin networks. Nature 445, 295-298.

[13] MacKintosh, F., Käs, J., and Janmey, P. (1995) Elasticity of Semiflexible Biopoly mer Networks. Phys. Rev. Lett. 75, 4425-4428.

[14] Bao, G., and Suresh, S. (2003) Cell and molecular mechanics of biological materials. Nat. Mater. 2, 715-725.

[15] Mendez-Vilas, A., and Díaz, J. (2010]) Microscopy. Science, technology, applications and education, Formatex, Badajoz.

[16] Kuznetsova, T. G., Starodubtseva, M. N., Yegorenkov, N. I., Chizhik, S. A., and Zhdanov, R. I. (2007) Atomic force microscopy probing of cell elasticity. Micron $38,824-833$.

[17] Rotsch, C., and Radmacher, M. (2000) Drug-induced changes of cytoskeletal structure and mechanics in fibroblasts: an atomic force microscopy study. Biophys. J. 78, 520-535.

[18] Janshoff, A., and Steinem, C. (2001) Scanning force microscopy of artificial membranes. ChemBioChem. 2, 798-808.

[19] Yang, Y., Wang, H., and Erie, D. A. (2003) Quantitative characterization of biomolecular assemblies and interactions using atomic force microscopy. Methods 29, 175-187.

[20] Müller, D. J., and Dufrêne, Y. F. (2008) Atomic force microscopy as a multifunctional molecular toolbox in nanobiotechnology. Nature Nanotech. 3, 261-269.

[21] Vinckier, A., and Semenza, G. (1998) Measuring elasticity of biological materials by atomic force microscopy. FEBS Lett. 430, 12-16.

[22] Sen, S., Subramanian, S., and Discher, D. E. (2005) Indentation and adhesive probing of a cell membrane with AFM: theoretical model and experiments. Biophys. J. 89, 3203-3213. 
[23] Lorenz, B., Mey, I., Steltenkamp, S., Fine, T., Rommel, C., Müller, M. M., Mai wald, A., Wegener, J., Steinem, C., and Janshoff, A. (2009) Elasticity Mapping of Pore-Suspending Native Cell Membranes. Small 5, 832-838.

[24] Steltenkamp, S., Müller, M. M., Deserno, M., Hennesthal, C., Steinem, C., and Janshoff, A. (2006) Mechanical properties of pore-spanning lipid bilayers probed by atomic force microscopy. Biophys. J. 91, 217-226.

[25] Mey, I., Stephan, M., Schmitt, E. K., Müller, M. M., Ben Amar, M., Steinem, C., and Janshoff, A. (2009) Local membrane mechanics of pore-spanning bilayers. J. Am. Chem. Soc. 131, 7031-7039.

[26] Mey, I., Steinem, C., and Janshoff, A. (2012) Biomimetic functionalization of porous substrates: towards model systems for cellular membranes. J. Mater. Chem.

[27] Kocun, M., Lazzara, T. D., Steinem, C., and Janshoff, A. (2011) Preparation of solvent-free, pore-spanning lipid bilayers: modeling the low tension of plasma membranes. Langmuir 27, 7672-7680.

[28] Schwamborn, M. (2012) Charakterisierung der Bindung von F-Aktin an Ezrin mittels Oberflächenplasmonenresonanz. Masterthesis, Göttingen.

[29] Tamm, L., and McConnell, H. (1985) Supported phospholipid bilayers. Biophys. J. $47,105-113$.

[30] Benda, A., Beneš, M., Mareček, V., Lhotský, A., Hermens, W. T., and Hof, M. (2003) How To Determine Diffusion Coefficients in Planar Phospholipid Systems by Con-focal Fluorescence Correlation Spectroscopy. Langmuir 19, 4120-4126.

[31] Przybylo, M., Sýkora, J., Humpolíčková, J., Benda, A., Zan, A., and Hof, M. (2006) Lipid Diffusion in Giant Unilamellar Vesicles Is More than 2 Times Faster than in Supported Phospholipid Bilayers under Identical Conditions. Langmuir 22, 9096-9099.

[32] Schmoller, K., Lieleg, O., and Bausch, A. (2009) Structural and Viscoelastic Properties of Actin/Filamin Networks: Cross-Linked versus Bundled Networks. Biophys. J. 97, 83-89. 
[33] Kasza, K., Broedersz, C., Koenderink, G., Lin, Y., Messner, W., Millman, E., Nakamura, F., Stossel, T., MacKintosh, F., and Weitz, D. (2010) Actin Filament Length Tunes Elasticity of Flexibly Cross-Linked Actin Networks. Biophys. J. 99, 1091-1100.

[34] Satcher, R., and Dewey, C. (1996) Theoretical estimates of mechanical properties of the endothelial cell cytoskeleton. Biophys. J. 71, 109-118.

[35] Wikström, A., Svedhem, S., Sivignon, M., and Kasemo, B. (2008) Real-Time QCM-D Monitoring of Electrostatically Driven Lipid Transfer between Two Lipid Bilayer Membranes. J. Phys. Chem. B 112, 14069-14074.

[36] Lingler, S., Rubinstein, I., Knoll, W., and Offenhäusser, A. (1997) Fusion of Small Unilamellar Lipid Vesicles to Alkanethiol and Thiolipid Self-Assembled Mono layers on Gold. Langmuir 13, 7085-7091.

[37] Naumann, R., Jonczyk, A., Kopp, R., van Esch, J., Ringsdorf, H., Knoll, W., and Gräber, P. (1995) Incorporation of Membrane Proteins in Solid-Supported Lipid Layers. Angew. Chem. Int. Ed. Engl 34, 2056-2058.

[38] Rossi, C., Homand, J., Bauche, C., Hamdi, H., Ladant, D., and Chopineau, J. (2003) Differential mechanisms for calcium-dependent protein/membrane association as evidenced from SPR-binding studies on supported biomimetic membranes. Bio-chemistry 42, 15273-15283.

[39] Gicquaud, C. (1995) Does Actin Bind to Membrane Lipids Under Conditions Compatible with Those Existing in Vivo? Biochem. Biophys. Res. Commun. 208, 1154-1158.

[40] Demé, B., Hess, D., Tristl, M., Lee, L.-T., and Sackmann, E. (2000) Binding of actin filaments to charged lipid monolayers: Film balance experiments combined with neutron reflectivity. Eur. Phys. J. E 2, 125.

[41] Barfoot, R. J., Sheikh, K. H., Johnson, B. R. G., Colyer, J., Miles, R. E., Jeuken, L. J. C., Bushby, R. J., and Evans, S. D. (2008) Minimal F-Actin Cytoskeletal System for Planar Supported Phospholipid Bilayers. Langmuir 24, 6827-6836. 
[42] Luisi, P. L., and Walde, P. (2000) Giant vesicles, Wiley, Chichester ;, New York.

[43] Rodriguez, N., Pincet, F., and Cribier, S. (2005) Giant vesicles formed by gentle hydration and electroformation: A comparison by fluorescence microscopy. Colloid Surface B 42, 125-130.

[44] Larsen, J., Hatzakis, N. S., and Stamou, D. (2011) Observation of Inhomogeneity in the Lipid Composition of Individual Nanoscale Liposomes. J. Am. Chem. Soc. 133, 10685-10687.

[45] Fine, T. (2009) Mapping the elastic response of epithelial apical cell membranes suspended across porous array. Dissertation, Mainz.

[46] Kocun, M., Mueller, W., Maskos, M., Mey, I., Geil, B., Steinem, C., and Janshoff, A. (2010) Viscoelasticity of pore-spanning polymer membranes derived from giant polymersomes. Soft Matter 6, 2508.

[47] Pontani, L.-L., van der Gucht, J., Salbreux, G., Heuvingh, J., Joanny, J.-F., and Sykes, C. (2009) Reconstitution of an Actin Cortex Inside a Liposome. Biophys. J. 96, 192-198.

[48] Limozin, L., Barmann, M., and Sackmann, E. (2003) On the organization of selfassembled actin networks in giant vesicles. Eur. Phys. J. E 10, 319-330.

[49] Cortese, J. D., Schwab, B., Frieden, C., and Elson, E. L. (1989) Actin polymerize tion induces a shape change in actin-containing vesicles. Proc. Natl. Acad. Sci. U.S.A $86,5773-5777$.

[50] Helfer, E., Harlepp, S., Bourdieu, L., Robert, J., MacKintosh, F., and Chatenay, D. (2001) Viscoelastic properties of actin-coated membranes. Phys. Rev. E 63.

[51] Mathur, A. B., Collinsworth, A. M., Reichert, W. M., Kraus, W. E., and Truskey, G. A. (2001) Endothelial, cardiac muscle and skeletal muscle exhibit different viscous and elastic properties as determined by atomic force microscopy. J. Biomech. 34, 1545-1553. 

The research field of protein adsorption dynamics on solid surfaces ${ }^{1-3}$ as well as protein-protein interactions that take place at solid interfaces has gained widespread scientific interest in the last decades. ${ }^{4-7}$ This is not surprising, as surface confined or more specifically membrane associated interactions between proteins are abundant throughout nature and can even involve a network of associated proteins. Understanding the mechanisms of these protein interactions, helps elucidating their specific biological role.

The aim of this work was to investigate the interaction of two different protein classes at the interface of lipid membranes to elucidate their binding affinity, specificity and their influence on membrane mechanics. Considering the cellular environment of the respective proteins, a great effort was undertaken to create artificial systems that could best mimic the natural environment of each protein.

In general, protein interactions can either be investigated by means of solution based or surface coupled techniques. ${ }^{8,9}$ It has often been criticized that the result obtained by either of the two possible options are not in accordance, although some groups report otherwise. ${ }^{10}$ It is easy to comprehend that membrane confined proteins might exhibit different characteristics and binding affinities as compared to proteins in solution.

Besides electrostatic and van der Waals forces ${ }^{11}$, the adsorption process of proteins at interfaces is mainly driven by an initial gain of entropy due to the loss of surface coupled water at the protein interface. ${ }^{4}$ A protein that is immobilized on a surface, however, loses two to four of the six degrees of freedom that they have in solution, caused by a reduction of translation and orientation possibilities. ${ }^{12}$ This reduction of protein entropy might alter the Gibb's enthalpy and dissociation constant of an interaction re- 
spectively. Also, conformational changes as a result of the adsorption process could be demonstrated in the past. ${ }^{13,14}$ Surface clustering, reorientation or other secondary processes (i.e. the Vroman effect) yet increase the intricacy of describing the impact of protein immobilization on the state of the adsorbed protein. . $^{15,16}$

In this work, however, coupling of the proteins to an artificial membrane is justified, if not superior to a solution based assay, as the interaction of naturally membrane coupled proteins or protein fragments are the matter of investigation.

In the first part of this work (chapter 4-6) a biosensor assay based on solid supported membranes (SSMs) was developed and adopted to investigate the interaction of C-terminal polycystin-2 (cPC2) with its putative interaction partners $C$-terminal polycystin-1 (cPC1) and PIGEA14. By means of the quartz crystal microbalance (QCM) technique, the interaction of cPC2 with cPC1 was quantified in terms of its $\mathrm{Ca}^{2+}$ dependence (chapter 5), while the interaction of cPC2 with PIGEA14 was investigated as a function of cPC2 pseudophosphorylation at Ser ${ }^{812}$ (chapter 6). In order to determine the impact of the respective variable and to allocate the interaction in terms of their biological relevance, dissociation constants and kinetic rate constants were extracted and compared.

Applying a model based on the scaled particle theory (SPT) implementing the random sequential adsorption (RSA) model ${ }^{17}$ and the mass transport of the protein to the sensor surface, we were able to evaluate kinetic data of the protein-protein interactions, including the most commonly effects that affect interaction processes that take place on surfaces. ${ }^{18,19,15}$

However, this assay does not provide information about conformational changes or other effects that might occur during the protein adsorption process. Although it is very challenging to determine all consequences that an adsorption process might have for a specific protein, there are some methods available to examine the state of a surface bound protein. By means of the atomic force microscopy (AFM) technique for instance, imaging of protein clusters on a flat surface such as a solid supported membrane (SSM) 
is possible. ${ }^{20}$ Conformational changes of the immobilized protein could be deduced from differential scanning calorimetry (DSC), circular dichroism (CD) or intrinsic fluorescence measurements. ${ }^{4,13}$ These methods, however, are only able to describe the state of the protein that is already bound to a surface. In situ monitoring of structural changes of the protein during the adsorption, which might affect the binding kinetics, is a task more complex.

The second part of the thesis deals with the interaction of filamentous actin (F-actin) with solid supported membranes lining a porous area and pore spanning membranes (chapter 7 and 8). Using anodic aluminum oxide (AAO) films, it was aimed to mimic the structure of cellular microvilli. The controlled adsorption of F-actin within and atop theses porous films respectively was studied by means of optical waveguide spectroscopy (OWS) and confocal laser scanning microscopy (CLSM, chapter 7). Besides the fact that a porous surface with the dimensions of AAO films is an adequate model system, if the regarding protein interaction takes place at a cellular system that is composed of ruffled structures, the main advantages of using AAO films lies in their optical transparency. Optical methods can be applied to investigate adsorption processes in a time resolved manner and enable one to discriminate whether a particle adsorbs only atop or within the pores. ${ }^{21,22}$ With this method in hand the adsorption of F-actin on the porous surfaces was controlled by using different functionalization strategies. Despite all the benefits of three dimensional surfaces, porous AAO films are not suited to study adsorption processes in a quantitative manner. This is because the adsorption of particles inside the pores is limited by their flux into the pores. ${ }^{23}$ Studying transport processes by means of other, macroporous substrates that base on porous $\mathrm{Si}_{3} \mathrm{~N}_{4}$ or other materials, offers the possibility of optical resolving an array of single pores. However, these substrates lack the advantage of optical transparency. ${ }^{24}$

The impact of a membrane attached F-actin network on the mechanical properties of pore spanning membranes (PSMs) was investigated by means of atomic force microscopy (AFM, chapter 8). Membranes that span an area in the micrometer range allow to investigate exclusively the tension of a membrane, being able to neglect the influence of 
the supporting pore rims. ${ }^{25,26}$ Other artificial membrane systems such as giant unilamellar vesicles (GUVs) have been utilized in the past to attach an F-actin network at the inner leaflet. ${ }^{27-29}$ However, it was first aimed in this work to determine the mechanical properties of F-actin bound to a planar membrane by means of AFM that, in contrast to cellular systems, is composed of an adjustable composition of the contributing proteins. An in vitro assay is advantageous compared to measuring cellular mechanics, as the impact of individual actin binding proteins (ABPs) on the membrane mechanics can be studied independently from one another. 


\subsection{References}

[1] The Biomedical Engineering Handbook. 2nd ed., CRC Press [Imprint]; Taylor \& Francis Group; Taylor \& Francis Group [Distributor], (1999) Abingdon, Flo rence.

[2] Rabe, M., Verdes, D., and Seeger, S. (2011) Understanding protein adsorption phenomena at solid surfaces. Adv Colloid Interface Sci 162, 87-106.

[3] Ball, V., Huetz, P., Elaissari, A., Cazenave, J. P., Voegel, J. C., and Schaaf, P. K. K. K. G. K. (1994) Kinetics of exchange processes in the adsorption of proteins on solid surfaces. Proc. Natl. Acad. Sci. U.S.A 91, 7330-7334.

[4] Hlady, V., and Buijs, J. (1996) Protein adsorption on solid surfaces. Curr. Optin. Biotech. 7, 72-77.

[5] Myszka, D. G. (1997) Kinetic analysis of macromolecular interactions using surface plasmon resonance biosensors. Curr. Opin. Biotechnol. 8, 50-57.

[6] Janshoff, A., and Steinem, C. (2005) Label-free detection of protein-ligand interactions by the quartz crystal microbalance. Method. Mol. Biol. 305, 47-64.

[7] Roos, H., Karlsson, R., Nilshans, H., and Persson, A. (1998) Thermodynamic analysis of protein interactions with biosensor technology. J. Mol. Recognit. 11, 204-210.

[8] Lakey, J. H., and Raggett, E. M. (1998) Measuring protein-protein interactions. Curr. Opin. Struct. Biol. 8, 119-123.

[9] Phizicky, E. M., and Fields, S. (1995) Protein-protein interactions: methods for detection and analysis. Microbiol. Rev. 59, 94-123. 
[10] Day, Y. S., Baird, C. L., Rich, R. L., and Myszka, D. G. (2002) Direct comparison of binding equilibrium, thermodynamic, and rate constants determined by surface and solution-based biophysical methods. Protein Sci. 11, 1017-1025.

[11] Roth, C. M., and Lenhoff, A. M. (1995) Electrostatic and van der Waals Contributions to Protein Adsorption: Comparison of Theory and Experiment. Langmuir $11,3500-3509$.

[12] Ben-Tal, N., Honig, B., Bagdassarian, C. K., and Ben-Shaul, A. (2000) Association entropy in adsorption processes. Biophys. J. 79, 1180-1187.

[13] Welzel, P. B. (2002) Investigation of adsorption-induced structural changes of proteins at solid/liquid interfaces by differential scanning calorimetry. Thermochim. Acta 382, 175-188.

[14] Steadman, B. L., Thompson, K. C., Middaugh, C. R., Matsuno, K., Vrona, S., Lawson, E. Q., and Lewis, R. V. (1992) The effects of surface adsorption on the thermal stability of proteins. Biotechnol. Bioeng. 40, 8-15.

[15] Ramsden, J. J. (1995) Puzzles and paradoxes in protein adsorption. Chem. Soc. $\operatorname{Rev} 24,73$.

[16] Ramsden, J., Bachmanova, G., and Archakov, A. (1994) Kinetic evidence for protein clustering at a surface. Phys. Rev. E 50, 5072-5076.

[17] Feder, J. (1980) Random sequential adsorption. J. Theor. Biol. 87, 237-254.

[18] Behn, D., Bosk, S., Hoffmeister, H., Janshoff, A., Witzgall, R., and Steinem, C. (2010) Quantifying the interaction of the C-terminal regions of polycystin-2 and polycystin-1 attached to a lipid bilayer by means of QCM. Biophys. Chem. 150, 47-53.

[19] Schuck, P., and Minton, A. P. (1996) Analysis of Mass Transport-Limited Binding Kinetics in Evanescent Wave Biosensors. Analytical Biochemistry 240, 262-272. 
[20] Herrig, A., Janke, M., Austermann, J., Gerke, V., Janshoff, A., and Steinem, C. (2006) Cooperative Adsorption of Ezrin on PIP 2 -Containing Membranes. Biochemistry 45, 13025-13034.

[21] Lazzara, T. D., Kliesch, T.-T., Janshoff, A., and Steinem, C. (2011) Orthogonal func- tionalization of nanoporous substrates: control of 3D surface functionality. ACS Appl Mater Interfaces 3, 1068-1076.

[22] Lazzara, T. D., Behn, D., Kliesch, T.-T., Janshoff, A., and Steinem, C. (2012) Phos pholipids as an alternative to direct covalent coupling: surface functionalization of nanoporous alumina for protein recognition and purification. J. Colloid. Interf. Sci. 366, 57-63.

[23] Lazzara, T. D., Mey, I., Steinem, C., and Janshoff, A. (2011) Benefits and Limita tions of Porous Substrates as Biosensors for Protein Adsorption. Anal. Chem 83, $5624-5630$.

[24] Mey, I., Steinem, C., and Janshoff, A. (2012) Biomimetic functionalization of porous substrates: towards model systems for cellular membranes. J. Mater. Chem.

[25] Mey, I., Stephan, M., Schmitt, E. K., Müller, M. M., Ben Amar, M., Steinem, C., and Janshoff, A. (2009) Local membrane mechanics of pore-spanning bilayers. J. Am. Chem. Soc. 131, 7031-7039.

[26] Kocun, M., Lazzara, T. D., Steinem, C., and Janshoff, A. (2011) Preparation of solvent-free, pore-spanning lipid bilayers: modeling the low tension of plasma membranes. Langmuir 27, 7672-7680.

[27] Pontani, L.-L., van der Gucht, J., Salbreux, G., Heuvingh, J., Joanny, J.-F., and Sykes, C. (2009) Reconstitution of an Actin Cortex Inside a Liposome. Biophys. J. 96, 192-198.

[28] Limozin, L., Barmann, M., and Sackmann, E. (2003) On the organization of selfassembled actin networks in giant vesicles. Eur. Phys. J. E 10, 319-330. 
270 I Conclusion and Outlook

[29] Cortese, J. D.; Schwab, B.; Frieden, C.; Elson, E. L. (1989) Actin polymerization induces a shape change in actin-containing vesicles. Proc. Natl. Acad. Sci. U.S.A $86,5773-5777$. 


\section{Appendix}

\subsection{Symbols}

$\mathrm{AAO}$

ABPs

ADPKD

AFM

Amp

APS

APTES

ATR-IR

Bodipy-PC

BRET

BSA

BSE

BVD anodic aluminum oxide

actin binding proteins

autosomal dominant polycystic kidney disease

atomic force microscopy

ampicillin

ammonium peroxosulfate

aminopropyl-triethoxysilane

attenuated total reflection infrared

2-(4,4-difluoro-5-methyl-4-bora-3a,4a-diaza-s-indacene-3-

dodecanoyl)-1-hexadecanoyl-sn-glycero-3-phosphocholine

bioluminescence resonance energy transfer

bovine serum albumin

backscattered electrons

Butterworth-van-Dyke 


$\begin{array}{ll}\text { Cam } & \text { chloramphenicol } \\ \text { C-ERMAD } & \text { C-terminal ERM association domain } \\ \text { CK2 } & \text { casein kinase 2 } \\ \text { CLSM } & \text { confocal laser scanning microscopy } \\ \text { Co-IP } & \text { co-immunoprecipitation } \\ \text { cPC1 } & \text { C-terminal polycystin-1 } \\ \text { cPC2 } & \text { C-terminal polycystin-2 } \\ \text { CPC2wt } & \text { C-terminal polycystin-2 wild type } \\ \text { cPC2S812D } & \text { C-terminal polycystin-2 mutant (Ser }{ }^{812} \text { replaced by Asp) } \\ \text { DIP } & \text { database of interacting proteins }\end{array}$

DOEPC 1,2-dioleoyl-sn-glycero-3-ethylphosphocholine

DOGS-NTA-Ni 1,2-dioleoyl-sn-glycero-3-[(N-(5-amino-1-carboxypentyl)iminodiacetic acid) succinyl] (nickel salt)

DOL $\quad$ degree of labeling

DOPC 1,2-dioleoyl-sn-glycero-3-phosphocholine

DTS dodecyl-trichlorosilane

E. coli Escherchia coli

ER endoplasmatic reticulum

F-actin filamentous actin 
FCS

FERM

FRAP

FRET

G-actin

GUVs

HEPES

His6

IPTG

IS

ITC

ITO

Kan

LB

LUVs

MBP

MG

MWCO

NA fluorescence correlation spectroscopy

$N$-terminal band four point one, ezrin, radixin, moesin

fluorescence recovery after photobleaching

fluorescence resonance energy transfer

globular actin

giant unilamellar vesicles

(4-(2-hydroxyethyl)-1-piperazineethanesulfonic acid )

hexahistidine

isopropyl $\beta$-d-1-thiogalactopyranoside

impedance spectroscopy

isothermal titration calorimetry

indium tin oxide

kanamycin

lysogeny broth

large unilamellar vesicles

maltose binding protein

Maxwell Garnett

molecular weight cut of

numerical aperture 
Ni-IDA nickel iminodiacetic acid

Ni-NTA nickel nitrilotriacetic acid

OD optical density

OWS optical waveguide spectroscopy

PACS-1, -2 phosphofurin acidic cluster sorting protein $1,-2$

PC1 polycystin-1

PC2 polycystin-2

PIGEA14 polycystin-2 interactor, Golgi- and endoplasmic reticulum-associated protein with a mass of $14 \mathrm{kDa}$

$\mathrm{PIP}_{2} \quad$ L- $\alpha$-phosphatidylinositol-4,5-bisphosphate

$\mathrm{PIP}_{2}$-TMR L- $\alpha$-phosphatidylinositol-4,5-bisphosphate-tetramethylrhodamine

POPC 1-palmitoyl-2-oleoyl-sn-glycero-3-phosphocholine

PSMs pore spanning membranes

QCM quartz crystal microbalance

ROI region of interest

RIfS reflectrometric interference spectroscopy

rpm rounds per minute

SAM self assembled monolayers

SDS sodium dodecylsulfate polyacrylamide 
SE

SEM

SPR

SPT

SSMs

SUVs

TAP

TE

TEMED

TIR

$\mathrm{TM}$

TRIS

TRP

TRPP2

$\mathrm{Y} 2 \mathrm{H}$ secondary electrons

scanning electron microscopy

surface plasmon resonance

scaled particle theory

solid supported membranes

small unilamellar vesicles

tandem affinity purification

transverse electric

$N, N, N^{\prime}, N^{\prime}$-tetramethylethylenediamine

total internal reflection

transverse magnetic

tris(hydroxymethyl)aminomethane

transient receptor potential

transient receptor potetial polycystin 2

yeast two hybrid

\subsection{Abbreviations}

A

surface area, absorbance 
$a$

C

c

$d$

D

E

$f$

F

$f_{0}$

$f_{\mathrm{e}}$

I

$k$

$K_{\mathrm{D}}$

$k_{\mathrm{s}}, k_{\mathrm{on}}, k_{\mathrm{off}}, k_{\mathrm{tr}}$

$l$

$L$

M

$m$ particle radius

capacitance

concentration

diameter, thickness

diffusion rate constant

electrical field, elasticity modulus

frequency

force

resonance frequency

frequency at equilibrium

intensity, current

spring constant

dissociation constant

kinetic rate constants of concentration dependent adsorption, adsorption, desorption and mass transport

length

inductance

molar mass

mass 


\begin{tabular}{|c|c|}
\hline$n$ & refractive index \\
\hline$R$ & resistance, reflectivity \\
\hline$r$ & radius \\
\hline$S$ & Sauerbrey constant \\
\hline$T$ & temperature \\
\hline$U$ & voltage \\
\hline Z & impedance \\
\hline$\varepsilon$ & extinction coefficient, dielectric constant \\
\hline$\Theta$ & surface coverage \\
\hline$\Theta_{\mathrm{i}}$ & angle of indicence \\
\hline$\lambda_{\mathrm{C}-\mathrm{C}}$ & lattice constant \\
\hline$\lambda_{\mathrm{em}}$ & emission wavelength \\
\hline$\lambda_{\mathrm{ex}}$ & excitation wavelength \\
\hline$\rho$ & density \\
\hline$\sigma$ & lateral membrane tension \\
\hline$\varphi(\omega)$ & phase shift \\
\hline$\omega$ & angular frequenecy \\
\hline
\end{tabular}




\subsection{Materials}

\subsubsection{Chemicals}

actin

Alexa Fluor488 C5-maleimide

Alexa Fluor488 phalloidin

Ampicillin

amylose resin

Bodipy-PC

BSA

Chloramphenicol

Complete-mini, EDTA-free

Coomassie Brillian Blue G250

DOEPC

DOGS-NTA-Ni

DOPC

DTS

Hellmanex

Kanamycin tebu-bio, Offenbach, Germany

Invitrogen, Eugene, USA

Invitrogen, Eugene, USA

Carl Roth GmbH, Karlsruhe, Germany

New England Biolabs, Frankfurt, Germany

Life Technologies GmbH, Darmstadt, Germany

Carl Roth GmbH, Karlsruhe, Germany

abcr GmbH, Karlsruhe, Germany

Roche, Mannheim, Germany

Carl Roth GmbH, Karsruhe

Avanti Polar Lipids, Alabaster, USA

Avanti Polar Lipids, Alabaster, USA

Avanti Polar Lipids, Alabaster, USA

Sigma Aldrich, Taufkirchen, Germany

Hellma, Müllheim, Germany

Sigma Aldrich, Taufkirchen, Germany 
mercaptoethanol

Mucasol

Ni-IDA-agarose

Ni-NTA-agarose

octanethiol

perylene

$\mathrm{PIP}_{2}$

$\mathrm{PIP}_{2}-\mathrm{TMR}$

POPC

Texas Red Maleimide
Sigma Aldrich, Taufkirchen, Germany

Merck, Darmstadt, Germany

Novagen, Darmstadt, Germany

Qiagen, Hilden, Germany

Merck, Darmstadt, Germany

Sigma Aldrich, Taufkirchen, Germany

Avanti Polar Lipids, Alabaster, USA

Echelon Bioscience Inc., Salt Lake City, USA

Avanti Polar Lipids, Alabaster, USA

Jena Bioscience, Jena, Germany

\subsubsection{Materials}

$5 \mathrm{MHz}$ AT-cut quartz crystals

aluminum foils

centrifugal ultrafiltration devices

copper stripe

dialysis tubes

double sided tape
KVG, Neckarbischofsheim, Germany

Goodfellow, Bad Nauheim, Germany

Sartorius, Göttingen, Germany

Präzisions Glas \& Optik GmbH, Iserlohn, Germany

Carl-Roth GmbH, Karsruhe, Germany

Tesa, Hamburg, Germany 
G-25 Sephadex gel filtration

column

ITO slides

Kalrez rings

LaSFN9 glasses

LWM Calibration Kit for SDS

MLCT D-lever

perestaltic pump tubings

porous silicon nitride substrates

quartz cuvette

Rotilabo® tubings (FEP)

Scotch vacuum tape

silicon wafer

two component epoxy glue

UV-curable, optical adhesive (NOA $83 \mathrm{H})$
GE Healthcare, Solingen, Germany

Präzisions Glas \& Optik GmbH, Iserlohn, Germany

DuPont, Wilmington, USA

Hellma Optik, Halle, Germany

GE Healthcare, München, Germany

AFM Probes, Camarillo, USA

Tygon Ismatec, Wertheim-Monfeld, Germany

fluXXion B.V, Eindhoven, Netherlands

Hellma Optik, Halle, Germany

Carl-Roth GmbH, Karsruhe, Germany

3M, Neuss, Germany

CrysTec, Berlin, Germany

UHU, Brühl, Germany

Norland Products, Cranbury USA). 


\subsubsection{Hardware}
Allegra $^{\mathrm{TM}}$ X-22R, Rotor SX4250
Beckman Coulter, Krefeld, Germany
Bal-Tec MCS610 thermal evaporation
Bal-Tec AG, Balzers (Liechtenstein)
Cary 50 UV spectrometer
Varian, Palto Alto, USA
Galaxy mini
VWR International, Darmstadt, Germany
Heraeus Fresco 17 Thermo
Waltham, USA
impedance spectrometer
Solatron Instruments, Farnborough, UK
and analyzer SI 1260
Jasco-810 spectrometer
Jasco, Gross-Umstadt, Germany
LEO supra-35 SEM microscope
Carl Zeiss GmbH, Jena, Germany
LSM 710
Carl Zeiss GmbH, Jena, Germany
MFP-3D stand alone AFM
Asylum Research, Santa Barbara, USA
MilliQ Elix 5
Millipore, Eschborn, Germany
MilliQ Gradient A10
Millipore, Eschborn, Germany
Optima $^{\mathrm{TM}} \mathrm{L}-90 \mathrm{~K}$, Rotor Ti70
Beckman Coulter, Krefeld, Germany
perestaltic pump
RegloDigital Ismateck, Wertheim, Mondfeld
pH meter
Calimatic 766 Knick, Berlin, Germany
pipettes
Eppendorf, Hamburg, Germany 
plasma cleaner PDC 32 G-2

QCM200

selection valve

Sonoplus HD 2070

SPR, Res-Tec RT 2005

sputter Coater 108 Auto

thermo mixer

Thermostat RE 104
Harrick Plasma, Ithaca, USA

Stanfort Research System Inc., Sunnyvale, USA

Upchurch Scientific, Oak Harbor, USA

Bandelin, Berlin, Germany

Resonant Technologies GmbH, Framersheim, Germany

Cressington Instruments, Watfort, UK

Eppendorf, Hamburg, Germany

Lauda, Königshafen, Germany 


\section{Curriculum Vitae}

Daniela Morick, née Behn

date of birth: $\quad$ August $5^{\text {th }}, 1983$

place of birth: Haldensleben

Citizenship: German

\section{Education}

$1996-2003$

Hannah-Arendt Gymnasium, Barsinghausen

$06 / 2003$

Allgemeine Hochschulreife (A-level equivalent) at the HannahArendt Gymnasium, Barsinghausen (final grade: 1.5)

Studies

$2003-2008$

Diploma Studies of Chemistry at the Georg-August-University, Göttingen

10/2005 Pre Diploma exam ("Vordiplom") at the Georg-August-University, Göttingen (final grade: 1.3 )

08/2006-02/2007 Internship at the University of Queensland, Brisbane, Australia (supervisor Prof. Craig Williams, title of project: Azadaralide-large scale synthesis studies)

09/2008

Final Diploma exam and thesis at the Georg-August-University, Göttingen (Prof. Claudia Steinem), title of diploma thesis: Interaction of C-Polycystin-1 with its specific interaction partners by means of quartz crystal microbalance under variation of the ionic strength, (final grade: 1.1)

11/2008-01/2013 Ph.D. thesis at the Georg-August-University, Göttingen (Prof. Dr. Claudia Steinem), title of thesis: In vitro studies of protein interactions on substrate supported artificial membranes 


\section{Publications}

Behn, D., Bosk, S., Hoffmeister, H., Janshoff, A., Witzgall, R., and Steinem, C. (2010) Quantifying the interaction of the C-terminal regions of polycystin-2 and polycystin-1 attached to a lipid bilayer by means of QCM. Biophys. Chem. 150, 47-53.

Lazzara, T. D., Behn, D., Kliesch, T.-T., Janshoff, A., and Steinem, C. (2012) Phospholipids as an alternative to direct covalent coupling: surface functionalization of nanoporous alumina for protein recognition and purification. J. Colloid. Interf. Sci. 366, 57-63.

Behn, D., Schatz, M., Hubrich, R., Hoffmeister, H., Witzgall, R., and Steinem, C. (2012) Phosphorylation of C-terminal polycystin-2 influences the interaction with PIGEA14: A QCM study based on solid supported membranes, Biochemistry, submitted

\section{Selected Presentations}

Behn, D., Hoffmeister, H., Witzgall, R., and C. Steinem (2009), C-Polycystin-2 and its specific interaction partners, poster presentation, 7th European Biophysical Congress (Genoa, Italy).

Behn ,D., Hoffmeister, H., Witzgall, R., and C. Steinem (2009) Polycystin-2 in the centre of a protein network: thermodynamics and kinetics of protein-protein interactions, oral presentation, $1^{\text {st }}$ Retreat of IMPRS school (Hofgeismar, Germany)

Behn, D., and C. Steinem (2011), Interaction of Filamentous Actinand Ezrin within Surface Modified Cylindrical Nanopores, poster presentation, 8th European Biophysical Congress (Budapest, Hungary).

\section{Scholarship}

02/2011-01/2013

Dorothea Schlözer scholarship 


\section{Danksagung}

Prof. Dr. Claudia Steinem danke ich für die stete Unterstützung und ihren Rückhalt während dieser Arbeit, für die Freiheit, die sie mir bei deren Durchführung einräumte und für die lehrreichen Gespräche und Diskussionen.

Den Mitgliedern meines Thesis Committees Prof. Dr. Kai Tittmann und Prof. Dr. Jörg Schroeder möchte ich für die Übernahme des Korreferats sowie ihre Diskussionsbereitschaft danken.

Prof. Dr. Ralf Witzgall danke ich für die unkomplizierte Kooperation und die Bereitstellung der Bakterienstämme. In diesem Zusammenhang möchte ich mich herzlich bei Larissa Osten für ihre Einführung in die Proteinisolierung bedanken!

Für ihre Unterstützung bei diversen Laborarbeiten möchte ich mich bei Jutta GerberNolte und Michaela Klingebiel bedanken. Den Sekretärinnen danke ich für die Hilfe bei den vielen organisatorischen Belangen.

Meinen Bachelor-, Master- und Diplomstudenten Michaela, Raphael, Markus und Miriam danke ich für ihre Motivation und den Eifer. Ihr habt tolle Arbeit geleistet und wesentlich zum Erfolg dieser Arbeit beigetragen!

Claudia, Ole, Julia, Christian, Jo und Ingo danke ich für das gewissenhafte Korrekturlesen! 
Dem gesamten Arbeitskreis danke ich für die schöne gemeinsame Zeit! Auch wenn sich die Zusammensetzung im Laufe der Jahre sehr gewandelt hat, konnte ich mich stets auf eure Hilfsbereitschaft und Unterstützung verlassen. Ohne die lustigen Ausflüge, Pausen und Messzeiten wären viele Rückschläge im Laboralltag nicht so leicht zu bewältigen gewesen.

Meinen Freunden aus Göttingen und Basche danke ich dafür, dass ihr immer da wart, wenn ich euch brauchte! Es ist schön, wenn man weiß, dass man überall ein Zuhause haben kann.

Ohne die Unterstützung meiner Familie wäre vieles nicht möglich gewesen! Besonders in der Endphase der Doktorarbeit haben meine Mutter und Schwiegermutter mir oft geholfen und sind eingesprungen, wenn Not am Mann war. Vielen Dank dafür!

Jo und Jonah, ihr habt das Größte überhaupt geleistet, indem ihr einfach da wart! Ihr habt mir gezeigt, dass es nicht vieler und oft tatsächlich keiner Worte bedarf, um zu erkennen, was wirklich Wert und Bestand hat! 